THE DECENTIA L PUBLIOATIONS OF THO UNIVERS SWY OF OHICAGO

SIUDIMS IN GMNDAD PHYSIOLOGX.

\title{
HACQUES IOAB
}


EX BIBLIOTHECA

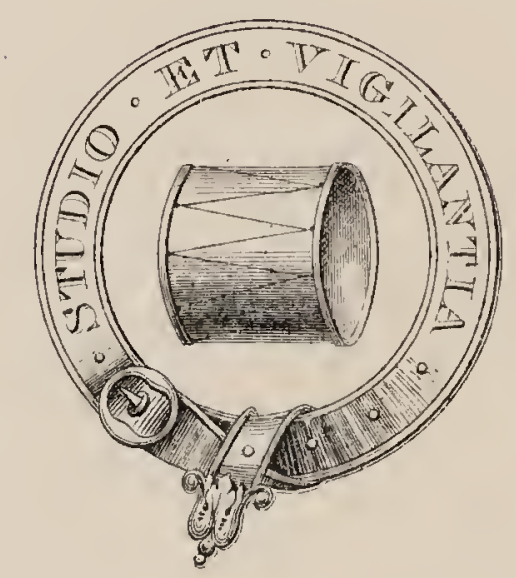

CAR. I. TABORIS. 
Med

K9852 


$$
\begin{aligned}
& \mathrm{g}_{1} \cdot 1 \cdot \mathrm{m} \\
& 5^{411}
\end{aligned}
$$




\section{THE DECENNIAL PUBLICATIONS OF THE UNIVERSITY OF CHICAGO}




\section{THE DECENNIAL PUBLICATIONS}

ISSUED IN COMMEMORATION OF THE COMPLETION OF THE FIRST TEN YEARS OF THE UNIVERSITY'S EXISTENCE

AUTHORIZED BY THE BOARD OF TRUSTEES ON THE RECOMMENDATION OF THE PRESIDENT AND SENATE

EDITED BY A COMMITTEE APPOINTED BY THE SENATE

EDWARD CAPPS

STARR WILLARD CUTTING ROLLIN D. SALISBURY

JAMES ROWLAND ANGELL WILLIAM I. THOMAS SHAILER MATHEWS

CARL DARLING BUCK FREDERIC IVES CARPENTER OSKAR BOLZA

JULIUS STIEGLITZ . JACQUES LOEB 
THESE VOLUMES ARE DEDICATED

TO THE MEN AND WOMEN

OF OUR TIME AND COUNTRY WHO BY WISE AND GENEROUS GIVING'

HAVE ENCOURAGED THE SEARCH AFTER TRUTH

IN ALL DEPARTMENTS OF KNOWLEDGE 
Digitized by the Internet Archive in 2019 with funding from Wellcome Library 
STUDIES IN GENERAL PHYSIOLOGY 



\title{
STUDIES IN GENERAL PHYSIOLOGY
}

\author{
$\mathrm{BY}$ \\ JACQUES LOEB \\ FORMERLY OF THE DEPARTMENT OF PHYSIOLOGY \\ NOW PROFESSOR OF PHYSIOLOGY IN THE \\ UNIVERSITY OF CALIFORNIA
}

THE DECENNIAL PUBLICATIONS

SECOND SERIES VOLUME XV

PART I

CHICAGO: THE UNIVERSITY OF CHICAGO PRESS

LONDON : T. FISHER UNWIN, PATERNOSTER SQUARE

1905 


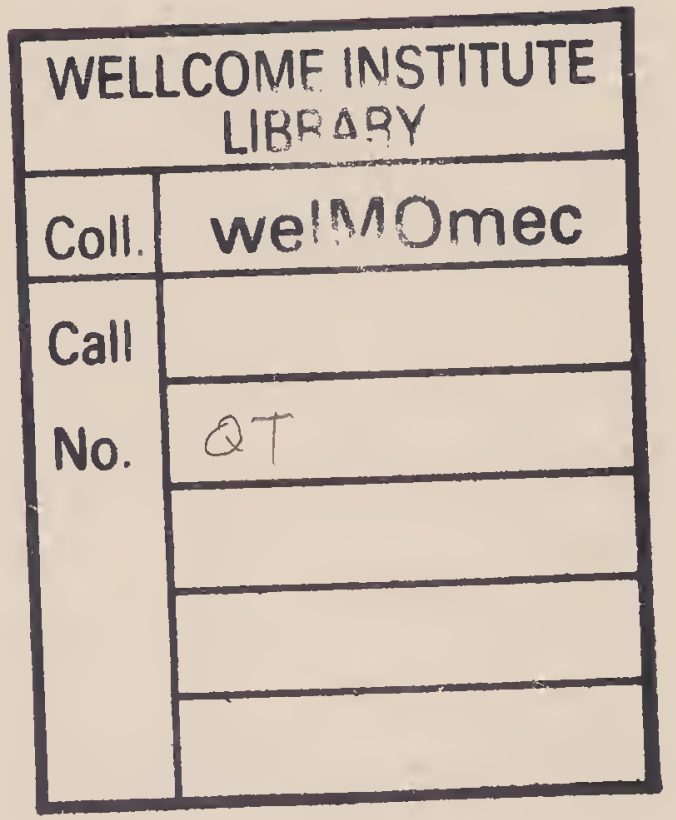




\section{PREFACE}

I SHould not have had the courage to offer these volumes to the public, had not requests repeatedly come to me from physicians and biologists to render my publications, which are widely scattered, more easily accessible. Therefore, when the editor of the "Decennial Publications of the University of Chicago" invited me to make a contribution to the series, I mentioned to him, not without hesitation, the idea of collecting and republishing my papers on General Physiology. Through his initiative and kind assistance the idea has been carried out.

No one will expect that a collection of papers on very diverse subjects can form attractive reading matter. Yet I may mention, by way of an apology, that, in spite of the diversity of topics, a single leading idea permeates all the papers of this collection, namely, that it is possible to get the life-phenomena under our control, and that such a control and nothing else is the aim of biology. Thus the reader will notice that in a series of these publications I have tried to find the agencies which determine unequivocally the direction of the motion of animals, and he will also notice that I consider a complete knowledge and control of these agencies the biological solution of the metaphysical problem of animal instinct and will. In taking up the problem of regeneration I started out with the idea of controlling these phenomena, and considered it my first aim to find means by which one organ could at desire be caused to grow in the place of another organ. Thus the experiments on heteromorphosis originated. As far as the problem of fertilization is concerned, it seemed to me that the first step toward its solution should consist in the attempt to produce larve artificially from unfertilized eggs in various classes of animals. 
It seemed desirable that the reader should be spared an undue amount of repetition, and for this reason a number of publications are omitted from this collection, and those printed are in many cases shortened. Among the papers which have been omitted are the preliminary notices and all those papers of which I am not the sole author. Occasionally I have made additions in the form of footnotes. Such footnotes have always been marked by the addition of [1903] at the end.

Only a small number of these papers appeared originally in English, namely, VII, XXI, XXVI-XXXV, and XXXVII. The other papers were translated from the German by Professor Martin H. Fischer, to whom I wish to express my sincere thanks. The credit as well as the responsibility for the translation belongs entirely to him. In the reading of the proof I was assisted by Dr. Fischer, Dr. Rogers, Dr. Bullot, and Dr. Bancroft. Mr. Rogers made the index for the first volume. To all these gentlemen my thanks are due.

Berkeley, California,

JACQUES Loeb.

October 14, 1904. 


\section{TABLE OF CONTENTS}

\section{PART I}

I. The Heliotropism of Animals and its Identity with the Heliotropism of Plants - $\quad$ - $\quad$ - $\quad$ - $\quad$ - 1

II. Further Investigations on the Heliotropism of Animals and its Identity with the Heliotropism of

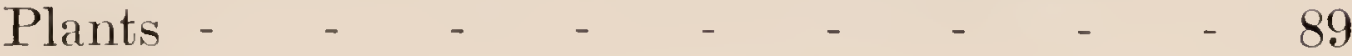

III. On Instinct and Will in Animals - - _ $\quad-107$

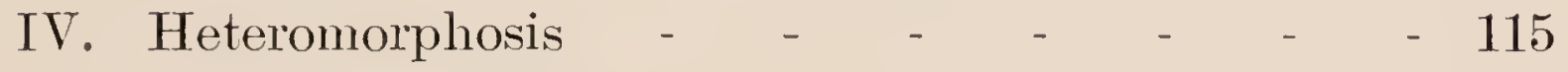

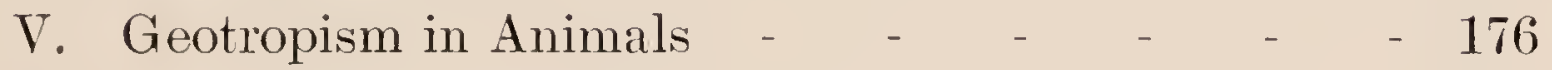

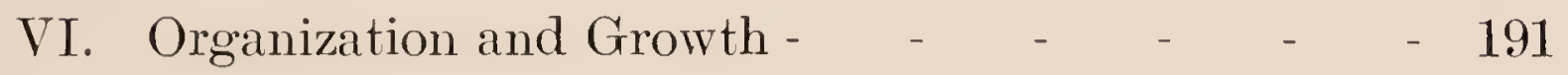

VII. Experiments on Cleavage - - $\quad$ - $\quad$ - $\quad$ - $\quad$ - 253

VIII. The Artificial Transformation of Positively Heliotropic Animals into Negatively Heliotropic and

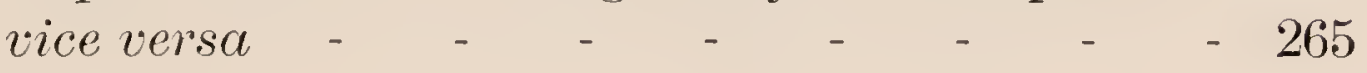

IX. On the Development of Fish Embryos with Sup-

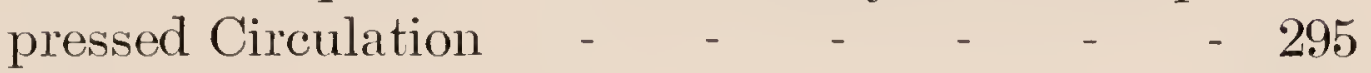

X. On a Simple Method of Producing from One Egg Two or More Embryos Which Are Grown Together - - $\quad$ - $\quad$ - $\quad$ - $\quad$ - $\quad$ - 303

XI. On the Relative Sensitiveness of Fish Embryos in Various Stages of Development to Lack of Oxygen

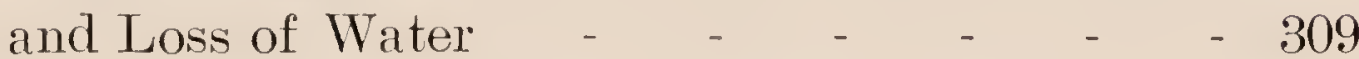

XII. On the Limits of Divisibility of Living Matter _ - 321

XIII. Remarks on Regeneration _ $\quad$ - $\quad$ - $\quad$ - $\quad$ - 338

XIV. Contributions to the Brain Physiology of Worms - 345

XV. The Physiological Effects of Lack of Oxygen - - 370 
PART II

XVI. The Influence of Light on the Development of

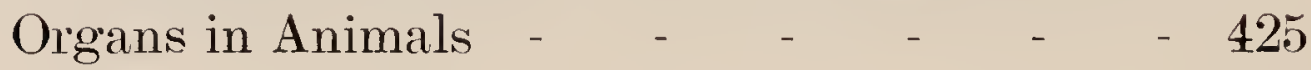

XVII. Has the Central Nervous System Any Influence upon the Metamorphosis of Larva?

XVIII. On the Theory of Galvanotropism - _ - 440

XIX. The Physiological Effects of Ions. I - - $\quad$ - 450

XX. On the Physiological Effects of Electrical Waves - 482

XXI. The Physiological Problems of Today - - _ 497

XXII. The Physiological Effects of Ions. II - - 501

XXIII. Why Is Regeneration of Protoplasmic Fragments without a Nucleus Difficult or Impossible? - 505

XXIV. On the Similarity between the Absorption of Water by Muscles and by Soaps - _ _ $\quad$ - $\quad 510$

XXV. On Ions Which Are Capable of Calling Forth Rhythmical Contractions in Skeletal Muscle - 518

XXVI. On the Nature of the Process of Fertilization and the Artificial Production of Normal Larvæ (Plutei) from the Unfertilized Eggs of the SeaUrchin - - $\quad$ - $\quad$ - $\quad$ - $\quad$ - $\quad$ - 539

XXVII. On Ion-Proteid Compounds and Their Rôle in the Mechanics of Life-Phenomena. - The Poisonous Character of a Pure NaCl Solution - - 544

XXVIII. On the Different Effects of Ions upon Myogenic and Neurogenic Rhythmical Contractions and upon Embryonic and Muscular Tissue - $\quad$ - 559

XXIX. On the Artificial Production of Normal Larvæ from the Unfertilized Eggs of the Sea-Urchin (Arbacia) - - $\quad$ - $\quad$ - $\quad$ - $\quad$ - $\quad$ - 576

XXX. On Artificial Parthenogenesis in Sea-Urchins - 624

XXXI. On the Transformation and Regeneration of Organs 627

XXXII. Further Experiments on Artificial Parthenogenesis and the Nature of the Process of Fertilization - 
XXXIII. Experiments on Artificial Parthenogenesis in Annelids (Chrotopterus) and the Nature of the Process of Fertilization - $\quad \ldots \quad \ldots \quad 646$

XXXIV. On an Apparently New Form of Muscular Irritability (Contact-Irritability?) Produced by Solutions of Salts (Preferably Sodium Salts) Whose Anions Are Liable to Form Insoluble Calcium Compounds - $\quad$ - $\quad$ - $\quad$ - $\quad$ - $\quad$ - $\quad$ - 692

XXXV. The Toxic and the Antitoxic Effects of Ions as a Function of Their Valency and Possibly Their Electrical Charge - $\quad$ - $\quad$ - $\quad$ - $\quad$ - $\quad$ - 708

XXXVI. Maturation, Natural Death, and the Prolongation of the Life of Unfertilized Starfish Eggs (Asterias Forbesii) and Their Significance for the Theory of Fertilization - $\quad$ - $\quad$ - $\quad$ - 728

XXXVII. On the Production and Suppression of Muscular Twitchings and Hypersensitiveness of the Skin by Electrolytes

XXXVIII. On the Methods and Sources of Error in the Experiments on Artificial Parthenogenesis - 766

INDEX 

PAR'T I 



\section{THE HELIOTROPISM OF ANIMALS AND ITS IDENTITY WITH THE HELIOTROPISM OF PLANTS ${ }^{1}$}

\section{INTRODUCTION}

I INTEND to show in the following pages that animal movements depend upon light in the same way as the movements of plants.

It is a well-known fact that animals, when light falls on them, move toward the source of light, like the moth, or move away from it, like the earthworm. It is also well known that certain plant organs have a tendency to turn toward or from the source of light when illuminated from one side only. While the conditions which govern the behavior of plants toward light have been well analyzed, especially by Sachs, little has been done to investigate the conditions upon which depend the movements of animals toward a source of light. It is the purpose of this paper to fill this gap, and to enumerate the facts which show that in reality the animal motions called forth by light depend upon the same circumstances as the motions which light produces in plants.

The effects of light which we intend to study are purely mechanical, inasmuch as they consist in changes in position, as well as in the direction and the sense of the progressive movements of living animals. Consequently we shall regard as essential such circumstances as can help to explain the mechanical effects of the light. These circumstances, as in the case of all stimulations, are of a double origin: first, those belonging to the stimulus - in this case the light; and,

1 Pamphlet, Würzburg, 1889. 
second, those belonging to the structure of the organism.

So far as the light is concerned, the circumstance which controls the orientation of the animal and the direction of its movements is the direction of the rays falling upon the animal. ${ }^{1} \quad$ The condition which is of importance on the part of the animal is the symmetrical shape of the body.

Sachs discovered that all plant organs which have a radial structure are orthotropic (this means that they bend, when light strikes them on one side, until their longitudinal axes lie in the direction of the rays of light), but that all dorsiventral structures are plagiotropic, $i$. $e$., they place their surfaces perpendicular to the rays of light. Symmetrically situated points at the surface possess a quantitatively and qualitatively equal irritability. In this way the organ of a plant is mechanically forced to orient itself in such a way that the rays of light strike symmetrical points at equal angles to the surface. If the plant, as for example the swarm spore of algæ, is capable of a progressive motion, it must of course, in order to maintain this position, move in the direction of the rays of light. This is, indeed, found to be the case.

I shall now show that quite generally in animals the direction of the rays of light controls also the direction of those movements which are caused by light; that, in addition, quite generally in animals their orientation depends

1 In these experiments it is presumed that the animals move under the influence of only one source of light. It is explicitly stated in this and the following papers that if there are several sources of light of unequal intensity, the light with the strongest intensity determines the orientation and direction of motion of the animal. Other possible complications are covered by the unequivocal statement, made and emphasized in this and the following papers on the same subject, that the main feature in all phenomena of heliotropism is the fact that symmetrical points of the photosensitive surface of the animal must be struck by the rays of light at the same angle. It is in full harmony with this fact that if two sources of light of equal intensity and distance act simultaneously upon a heliotropic animal, the animal puts its median plane at right angles to the line connecting the two sources of light. This fact was not only known to me, but had been demonstrated by me on the larvæ of flies as early as 1887, in Würzburg, and often enough since. These facts seem to have escaped several of my critics. [1903] 
on the form of the body in so far as dorsiventral animals move with their median planes in the direction of the rays of light, in which position the rays fall upon symmetrically situated points of the surface of their bodies at nearly equal angles. In this way the fact that a moth flies into a flame turns out to be the same mechanical process as that by which the axis of the stem of a plant puts itself in the direction of the rays of light. In both cases, however-in the fatal flight of the moth as well as in the orientation of plants one point remains unexplained, namely: how can the light so change the state of the protoplasm as to bring about the mechanical effects just mentioned? At present we are not able to form a clear idea of this.

A second condition which has a determining influence upon the mechanical effects of light on plants is the refrangibility of the rays. Sachs has shown that it is chiefly the more refrangible rays which are able to bring about movements in plant organisms. We shall see that quite generally the more refrangible rays are also more effective mechanically in the animal kingdom.

Thirdly, we shall prove that the orientation of animals as well as of plants takes place when the intensity of the light remains constant. Very often we observe, for example in our eyes, that a change in the intensity of the light acts as a stimulus. In addition to these essential considerations of the effects of light in the animal kingdom, the following factors play a rôle, namely:

Fourthly, light causes the orientation of animals (as well as of plants) only within certain limits of intensity. Fifthly, temperature influences the movements of orientation in animals and plants toward light — which is true for all phenomena of stimulation.

To sum up: The conditions which control the movements of animals toward light are identical, point for point, 
with those which have been shown to be of paramount influence in plants.

Aside from the problem of proving by suitable experiments the stated propositions, it is also necessary for us to show what rôle the orientation toward the light plays in the economy of life of an animal. I shall therefore first describe the experimental proofs of the identity of animal heliotropism with plant heliotropism, and then snow by individual examples what rôle heliotropism plays in the economy of life of animals. To discuss the latter point it will be necessary also to describe briefly the other forms of irritability possessed by an animal.

In a short article which appeared in January, 1888, I described the principal laws upon which depends the orientation of animals to light, and the identity of these laws with those governing plant heliotropism. ${ }^{1}$

\section{THE ESSENTIAL PHENOMENA AND LAWS OF HELIOTROPISM} IN PLANTS

Assuming that the reader is acquainted with the orientation of plants toward a source of light, it will suffice at this place to call attention briefly to the essential facts which bear upon our subject. In so doing I shall follow the presentation given by J. von Sachs in his lectures on plant physiology. ${ }^{2}$

Straight stems or roots of growing plants bend when light falls on them on one side only, or with greater intensity on one side than on the other, until their tips lie in the direction of the rays of light. Those organs which turn toward the source of light are called positively heliotropic; those which turn from the light, negatively heliotropic.

1 "Die Orientierung der Thiere gegen das Licht (thierischer Heliotropismus)," Sitzungsberichte der Würzburger physikalisch-medicinischen Gesellschaft, January, 1888.

2 Vorlesungen über Pflanzen-Physiologie, 2d ed. (Leipzig, 1887). 
It was formerly believed that the bending of the positively heliotropic parts of plants was due to the fact that the side which was turned away from the light grew more rapidly, because plants when brought into the dark at first grow more rapidly than they do in the light. But it was proved in Sachs's laboratory that negatively heliotropic organs also grow more rapidly in the dark. Because of the similarity of the geotropic and heliotropic movement in plants, Sachs came to the conclusion that the direction in which the rays of light penetrate the plant tissue determines the orientation of the plant toward light. He also proved that not all the rays of the visible sun spectrum bring about heliotropic movements, but only, or at least chiefly, the more refrangible rays. The less refrangible rays, which are of importance in assimilation, are ineffective heliotropically. If the light be previously passed through a dark-blue ammoniacal solution of copper, which absorbs all the red, yellow, and a part of the green rays, the heliotropic bending occurs in the same way as in completely white light. If, however, the light passes through a saturated solution of potassium bichromate, which lets through only red, yellow, and a part of the green rays, "the heliotropic shoots remain straight and vertical, no matter how intense the light is which passes through the solution." Finally, if the light "is passed through a solution of quinine sulphate, the fluorescence of which completely absorbs the ultra-violet rays, the heliotropic curvatures nevertheless appear - a proof that they are caused principally by the visible blue and violet rays."

The best proof of the theory that the direction of the rays of light controls the orientation of plants was found by studying freely moving plant organs, the swarm-spores of algæ. These swarm-spores make progressive movements like animals, and Strasburger ${ }^{10}$ proved that they move in the

1 STRASBURGER, Wirkung des Lichtes und der Würme auf Schwärmsporen (Jena, 1878). 
direction of the rays, to or from the source of light. The more refrangible rays alone exercise this effect on the swarmspores. They behave in the light which has passed through an ammoniacal solution of copper just as in diffuse daylight. On the other hand, they are not affected by light which has passed through a potassium bichromate solution, by light from a sodium flame, or by the light coming through ruby glass.

The chlorophyll-bearing protoplasm of cells moves under the influence of light. ${ }^{1}$ The chloroplasts of a thread alga, Mesocarpus, turn "their broad surfaces toward the sky so that the rays fall upon them at right angles. If the direction of the rays is changed, the chloroplasts turn so that their broad surfaces are again at right angles to the rays. Direct sunlight, however, causes the chloroplasts to assume another position - they place their surfaces parallel to the rays which strike them."

According to modern plant physiology, the whole protoplasm of a multicellular plant is to be conceived of as a continuous mass, as a single protoplasmic body. ${ }^{2}$ More recent investigations have shown that when a plant organ is illuminated, that side of the organ which becomes concave from the effect of the light becomes rich in protoplasm, while the opposite convex side becomes poor. ${ }^{3}$ Multicellular organs behave in this regard like unicellular ones. Thus it appears that the light forces the protoplasmic mass to move in such a way that positively heliotropic protoplasm wanders to the side of the organ which is turned toward the light, while negatively heliotropic protoplasm wanders to the opposite side. ${ }^{4}$ Should it turn out that this phenomenon really occurs

1 SтAнL, Botanische Zeitung, 1880.

2 Sachs, loc. cit., p. 94.

3 Wortmann expressed his observations in this way. It is possible that in reality protoplasm on the concave side is only more opaque than on the opposite side. This difference in optical appearance may simply be the expression of a difference in the size of the colloidal particles. [1903]

4 See Wortmans, Botanische Zeitung, 1887. 
in all cases, it would prove that the protoplasm of a multicellular plant behaves just like the naked, creeping plasmodium, which is also heliotropically irritable.

III. SUMMARY OF THE MECHANICAL EFFECTS OF LIGHT IN THE ANIMAL KINGDOM WHICH ARE THUS FAR KNOWN

I shall in this chapter summarize briefly the facts and views in regard to the movements of animals under the influence of light, so far as they are known up to the present time. These may be divided into three groups:

1. Casual observations of the older authors (Réaumur, Trembley). These are unprejudiced records of simple observations.

2. Modern investigations on the effects of light from an anthropomorphic standpoint. The movements of animals are not attributed to mechanical causes, but to supposed human sensations of the animals.

3. Investigations according to the method of Sachs, which, however, have been applied only to Protozoa. The lastnamed observations are the most important in these three groups.

The earliest account of the effects of light on animals which I have found in the literature is by Réaumur. ${ }^{1} \mathrm{He}$ found that moths which are attracted by the candle flame "do not fly from flower to flower during the day." Since he saw chiefly the males fly into the flame, he raised the question as to whether or not the female moths emit light like glowworms. "Do not the females of the nocturnal Lepidoptera emit a light too feeble to make an impression on our eyes, but sufficiently strong to act on those of their males?" $\mathrm{He}$ had observed, evidently, that the males of the glow-worm which are attracted by the light to the aboral end of the females likewise fly into the light. Réaumur was, moreover,

1 RÉAUMUR, Hémoires pour servir à l'histoire des insectes, Vol. I, 1, p. 330 (Amsterdam, 1748). 
convinced that the glow-worm living in the woods could see. He made a glass window in a tree in which such worms lived and noticed that the animals gave a start upon the approach of a burning candle.

Trembley made far better experiments. ${ }^{1}$ He found that "water fleas" can be driven around in a circle by a moving candle:

By the light of a wax taper I observed polyps to which during the day I had given many water fleas; in the evening there were left in the glass some which the polyps had not consumed. I noticed that most of them had collected on the side toward the candle. I changed the position of the taper, and they followed it. As I had moved its position repeatedly, and each time had seen that the water fleas followed it, I moved the taper slowly around the glass without stopping. They followed, and thus made several trips around it. I have had the opportunity of repeating this experiment several times.

Trembley's observations on the effect of light on Hydra were made with great care. After he had repeatedly observed that the polyps moved to the "brightest" side of the glass, he placed "a glass containing many green polyps in a case which had an opening on one side about opposite the middle of the glass." He reports as follows concerning their behavior:

When I placed the glass so that the opening in the case was turned to the light, the polyps always migrated toward that side of the glass which was opposite this opening, in such a way that together they made the figure of a gable. I often turned the glass around, and after several days I observed the polyps again at the opening arranged as before (in the form of a gable). To vary the experiment still further, I fixed the dark case so that the opening was at times straight, at other times inverted, and again the polyps arranged themselves together.

After he had discovered that polyps which had been cut in two could "move, eat, and multiply," he tried to see "whether

1 Trembley, Abhandlungen zur Geschichte einer Polypenart, transl. by Götze (Quedlinburg, 1791). 
these pieces would turn toward the light in the same way as the undivided polyps." He cut a number of polyps in two: the anterior halves he placed in one glass, the posterior halves in another. He found "in oft-repeated experiments that the animals in both glasses collected in the brightest regions in the glass."

These are, as far as I know, the only extended observations to be found in the old physiological literature of the effects of light upon animals. For a long time no further study of the effects of light upon animals was made. Johannes Müller mentions, in the preface to his Physiologic dos Gesichtssinnes, that he made "investigations on the influence of colored light on the vital phenomena of plants and animals," but, as far as I know, the results of his investigations were never published.

The modern anthropomorphic observations were introduced by Paul Bert. Bert raised the question: Do all animals see the same rays that we see ${ }^{11}$ He meant to ask whether all rays of the visible sun spectrum are able to bring about animal movements. An experiment with Daphnia pulex was sufficient for Bert to settle this question. He projected a spectrum and found that the animals became restless in all positions of the visible spectrum:

Mes daphnies erraient dispersées d'une manière à peu près Egale dans toute l'étendue du vase obscur, lorsque soudain je fis tomber sur la fente un rayon coloré, un rayon vert. Aussitôt elles s'agitèrent, se groupèrent toutes dans la direction de la trainée lumineuse et un très-grand nombre s'en vint se heurter, montant et descendant sans relâche contre la paroi qui recevait la lumière. Or, un semblable résultat fut obtenu pour toutes les régions du spectre visible. Le rouge, le jaune, le bleu, le violet même attiraient les daphnies. Seulement il fut facile de remarquer, qu'elles accouraient beaucoup plus rapidement au jaune ou au vert qu'à toute antre couleur.

${ }^{1}$ BerT, Archives de physiologie, 1869. 
On either side of the spectrum the animals remained at rest.

In addition to this, Bert made another experiment. $\mathrm{He}$ had a spectrum projected on a trough, and observed how the animals distributed themselves over the different parts of the spectrum.

L’immense majorité se plaça dans le jaune, le vert, l'orange; une assez grande quantité se voyaient encore dans le rouge, un certain nombre dans le bleu, quelques-unes de plus en plus rares à mesure qu'on s'éloignait dans les régions plus réfrangibles du violet, au delà du rouge, au delà de l'ultra-violet; dans les régions invisibles, en un mot, on n'en trouvait que d'isolées en promenade accidentelle.

From these facts Bert concluded that Daphnia behaves in the spectrum much as a man would, who, when reading a book, would move into the brightest part of the spectrum, into the yellow light.

Lubbock repeated Bert's experiment on Daphnia. ${ }^{1}$ Onehalf of a dish was covered by a yellow screen; the other half was left uncovered. In the uncovered half $\mathbf{1 , 9 0 4}$ animals collected, while 3,096 gathered under the yellow screen. From this Lubbock concludes that Daphnia has a "preference" for "yellow." But one would suppose that in the uncovered part of the dish there was at least as much yellow light as under the yellow screen; or did the majority "hate" the blue light?

When Lubbock covered one-half of the trough with blue glass and left the other uncovered, he found 2,046 animals under the blue glass, and 2,954 in the uncovered part of the trough. Whether one is to conclude from this that blue light is in the sense of Lubbock "disagreeable" to Daphnia is not stated. When half of the trough was covered with red glass, there collected 1,928 animals under the red glass, while 3,072 collected in the uncovered por-

1 Lubвоск, "Dio Sinne und das geistige Leben der Thiere," Internationale wissenschaftliche Bibliothek, Vol. LXVII (1889). 
tions of the dish. When half of the vessel was covered with an opaque porcelain screen, Lubbock found 2,0 48 animals collected under it, and 2,932 animals in the uncovered half. From these and similar experiments Lubbock concludes that the animals have a decided preference for yellow light.

I also have made some experiments on the effects of rays of different refrangibility on Daphnia, and found that when the more refrangible rays (blue and violet) fell upon the animals they hastened to the source of light and moved up and down on the light side of the vessel. When I made the same experiment with the less refrangible rays, the effect was weak or did not take place at all. The result conforms with other facts which are to be described later. I shall, therefore, not revert to the Daphnia and their alleged "preference for yellow."

Lubbock has employed a similar method in his experiments on wingless ants $;^{1}$ these, however, led to much more fruitful results than his experiments on Daphnia. In an experiment in which a vessel was covered with strips of red, green, yellow, and violet glass he found that 890 animals collected under the red glass, 544 under the green, 495 under the yellow, and only 5 under the violet. There is no doubt in this case that the animals collected under those glasses where they were struck by the less refrangible rays. Other experiments showed that red glass acts like an opaque body.

The observation of Lubbock that ants avoid the ultraviolet part of the spectrum is also worthy of note. For the sake of completeness the experiments of Lubbock on bees and wasps must be mentioned, in which it was found that under otherwise similar conditions blue objects smeared with honey were preferred to those of another color.

${ }^{1}$ Luввоск, "Ameisen, Bienen und Wespen," ibid., 1883. 
The most extended experiments on the influence of light on the orientation of animals were made by Graber. ${ }^{1}$ His "comparative studies on light-sensations" (Vergleichende Licht-Gefühl-Studien), as he called his investigations, cover about fifty species. His method is that followed by Lubbock.

The faultiness of this method and the errors of interpretation of the results obtained stand out more clearly in Graber's writings than in Lubbock's. Graber covers onehalf of a vessel with a partially or completely opaque screen, and after a time notes how the animals are distributed in the vessel. If most of the animals are under the opaque screen, Graber says that they are "fond of the dark" and "hate the light;" or in the reverse case, that they are "fond of the light" or of "the white" and "hate the dark." He therefore uses the conceptions of "white" or "bright" and "dark," which designate certain effects of light upon a human being for the conceptions of great or small intensity of the light; and in saying that animals which "prefer the light" also "hate the darkness" he makes a second mistake in that he maintains that strong and weak light have opposite effects. We shall see, however, that these effects are similar and differ only in degree. He makes the same mistake in experimenting on rays of different refrangibility. The most important among the facts observed by him in this connection is this, that animals which "prefer the light" with a few exceptions also "prefer" blue, while those which "hate the light" "prefer" red. His ideas are expressed in the following remarks, which, however, I do not fully understand:

The question arises as to the cause of this truly striking relation between the love for white light and for blue light, on the one hand, and between the dislike for white light and for blue light,

1 Grundlinien zur Erforschung des Helligkeits. und Farbensinnes der Thiere (Prag, 1884). 
on the other hand. If the law had reference only to white light, and not also to colored light-red, blue, etc.-which is, however, by no means always the case, one mightat first be inclined to believe that the animals which prefer red avoid mixed light because it contains many of the hated short waves of the blue and violet light; for this very reason it would be more agreeable than dim light to the animals which prefer blue, for dim mixed light is poor in all rays, and therefore also in blue. Yet the objection might be raised against this explanation that mixed light contains as much red for those animals which prefer red as it contains blue for those animals which prefer blue. Yet this objection could again be weakened by the assumption that, since the animals which prefer red also prefer darkness, they prefer a minus of their chosen color to a plus of the color they dislike.

Graber finally considers it best "to await further investigations in a field where great darkness still prevails." We see that Graber in regard to the effects of monochromatic light again establishes a contrast in effects where, as we shall see, a similarity exists. Graber was prevented from correctly interpreting his results by attributing the movements of animals to sensations instead of to physical causes. If he had given up the anthropomorphic standpoint, he would soon have discovered that his experiments show that the more refrangible rays are more effective in causing the orientation of an animal than the less refrangible ones.

In none of the investigations of Bert, Lubbock, or Graber has the influence of the direction of the rays on the orientation been studied. Graber, for example, took it for granted that an animal moves to the light because, as he expressed it, "it is fond of the light" or "the white." If it moves in the opposite direction, it "is fond of the dark." Lubbock remarks incidentally that "ants do not like light in their nests, probably because they do not deem it safe."

This sums up the opinions and results of the authors who sought to explain anthropomorphically the phenomena which interest us here. 
Finally, I have to mention the heliotropic investigations on Infusoria which were made along the lines mapped out by Sachs. To bring these investigations before the reader I shall describe the more important observations which have been made on Euglena. The influence of the direction of the rays of light on these Infusoria was first demonstrated by Stahl: ${ }^{1}$

Those individuals which did not swim about freely remained with their pointed posterior ends attached to the cover-glass or to other objects, while their free anterior ends were, according to conditions, either turned toward or away from the source of light. The longitudinal axes of both the motile and sessile Euglenæ coincided as nearly as possible with the direction of the rays of light. The motionless ones behaved like the free-swimming ones whenever the direction or intensity of the light was suddenly changed, except that they reacted more slowly. If, for example, the glass slip was suddenly rotated through an angle of $180^{\circ}$, the position which the animals occupied originally with reference to the source of light was slowly reassumed, while the swimming individuals left their former path and moved in the original direction toward the light immediately after a change in its direction.

Engelmann studied in Euglena the relation between the effect of the rays of light and their refrangibility. ${ }^{2}$ After he had established the fact that when a drop of Euglenæ is only partially illuminated the animals gradually accumulate in the lighted area, he brought the animals into a microspectrum. Here they collected on the more refrangible side of the spectrum. The orientation of Euglena therefore depends on the direction of the rays, and especially on that of the more refrangible ones. It must finally be mentioned that the anterior ends of the Infusoriæ are most sensitive to light; yet the pigment spot is not, as might be supposed, the most sensitive, but the colorless protoplasm in front of this.

Besides these direct effects of light in phenomena of 
orientation, which alone interest us here, there are also certain indirect effects on the orientation of low forms of life. These were also first observed by Engelmann. When the supply of oxygen is cut off from certain chlorophyll-bearing organisms, they remain in that part of the spectrum in which assimilation takes place. In water with its normal amount of oxygen, as Engelmann found, Stentor viridis, Bursaria, and the green slipper animalculæ do not react to light. 'If, however, the supply of oxygen from without is interfered with, "the insufficient supply can be compensated for by a production of oxygen by the chlorophyll granules within the mesoplasm." Under these conditions the animals return to the light side of the drop when they accidentally get into the shady part. When the animals are brought into a microspectrum, they collect in those regions which promote assimilation. The opposite effect takes place, however, when the supply of oxygen from without exceeds the normal. When Engelmann passed a stream of pure oxygen through the water, the animals moved from the lighted into the shaded part of the drop.

Such an indirect orientation toward light as is determined by assimilation is shown also in the behavior of the purple bacteria. $^{2}$ These, as Engelmann found, collect in those regions of the spectrum which are most absorbed by the coloring matter of the bacteria.

These are the most important facts which up to this time are known concerning the influence of light on the orientation of animals. Thus far only the observations made on Infusoria are sufficient to warrant the conclusion that animal movements depend on light in the same way as the movements of plants. In the rest of the animal kingdom either the facts necessary for this conclusion are lacking, or false statements and conceptions are prevalent. So far as 
the latter are concerned, it is wrong, as we shall see, to say that certain animals "are fond of the light" and seek those regions in space where light is most intense, while others "are fond of the dark" and betake themselves to those regions which are darkest. In contradiction of this idea I shall prove that the direction of the progressive heliotropic movements of animals is determined solely by the direction of the rays, no matter whether the animals move from regions in which light is less intense to those in which it is more intense, or vice versa.

Further than this, it is fundamentally wrong to say that an assumed "preference for color" determines the orientation of animals toward rays of different refrangibilities; that, as Graber says, the animals which "are fond of blue" "hate red," and that those which "are fond of red" "hate blue." In contradiction of this idea I shall prove that there are no animals which "are fond of" red or "hate" blue, but only such as move toward a source of light or away from it; and that these movements occur in the same way under the influence of the more refrangible rays as under that of the less refrangible rays, only with this purely quantitative difference, that the more refrangible rays, as in plants, are much more effective than the less refrangible ones, which usually have no effect.

I consider it inadvisable to represent the movements observed in animals as the expression of a "color preference," or a "color sensation," of a "pleasurable" or "unpleasurable sensation," as do most animal physiologists and zoölogists who have studied the effects of light in the animal kingdom. I do not propose to base an analysis of the movements of animals on such hypothetical, anthropomorphic sensations and feelings, but on such conditions as determine the course of phenomena in inanimate nature as well. Real natural science began when, instead of fabulizing over the 
nature of gravitation, men determined accurately the details of the movement of falling stones, of pendulums, etc., and described them in the most simple and definite terms. In biology, especially in regard to the mechanical effects of light which concern us here, the task of the investigator can only be to determine and describe the circumstances upon which depend the movements of animals under the influence of light.

IV. REMARKS ON THE METHOD OF EXPERIMENTATION.- THE HELIOTROPISM OF AN ANIMAL USUALLY BECOMES EVIDENT ONLY AT A DEFINITE EPOCH IN ITS EXISTENCE.-THE HELIOTROPISM OF AN ANIMAL CAN EASILY BE OBSCURED BY A SPECIAL FORM OF CONTACT-IRRITABILITY

The facts which I have to prove are so simple that almost all technical apparatus can be dispensed with. If one attempts to demonstrate that the orientation of the animals is controlled by the direction of the rays of light, care must be taken that light falls upon the animals from only one side. To accomplish this it is sufficient to carry on the experiments in a room which is lighted from one side only. Since the animals with which we are dealing in this discussion are dorsiventral and place their median planes in the direction of the rays of light, progressive movements are possible in only two directions-either toward the source of light (when they will be called positively heliotropic), or away from the source of light (in which case they will be called negatively heliotropic). ${ }^{1}$

Diffuse daylight was used as the source of light, and only where specially mentioned was sunlight employed.

1 Soine botanists designate the movements of motile plant organisms toward a source of light as "phototactic," in contrast to the "heliotropic" movements of sessile plants. Since the observations of Sachs, Stahl, and Wortmann, however, loave no room for doubt that the processes are identical in both cases, it seems to me that this separation is not justified. Otherwise a "phototactic" animal ought to become "heliotropic" when its progressive movements are prevented. For this reason $\mathrm{I}$ use the same term for similar processes. (See Wortmann, Botanische Zeitung, 1887.) 
There are two methods by which the second fact, that only the more refrangible rays bring about orientation, can be proved, namely, by experimenting with prismatic spectra or with colored screens.

All authors who have studied the behavior of plants behind colored screens have obtained the same result - that it is only, or more especially, the more refrangible rays which are heliotropically active. Studies on the behavior of plants in prismatic spectra have led to harmonious results, in so far as they confirm the gross results obtained by using colored screens; yet opinions differ as to the efficacy of the more limited portions of the spectrum. Since for the present I wish to show only that the laws governing the orientation of an animal toward light correspond to the laws governing the orientation of plants toward the same stimulus, it was necessary to use as a basis the really established data of plant physiology, and I therefore shall confine myself to the proof of the fact that the more refrangible rays of the spectrum are exclusively, or almost exclusively, effective. To do this I proceeded as is usual in plant physiology. In order to have only the less refrangible rays act on the animals, I passed the diffuse daylight through a solution of potassium bichromate or ruby glass; to study the influence of the more refrangible rays, I chose cobalt glass or an ammoniacal solution of copper. The screens were examined spectroscopically. The dark-red glass which I used completely absorbed the more refrangible rays, and let through only the red, yellow, and a part of the green rays. The dark-blue glass absorbed the less refrangible red and yellow and a part of the green rays, with the exception of a small region in the outer red. Since, however, the heliotropic phenomena appear only weakly or not at all behind dark-red glass, while they occur just as in diffuse daylight behind dark-blue glass, the few red rays which penetrate the dark-blue glass cannot be 
responsible for the heliotropic phenomena which take place so energetically behind this screen, but can be due only to the activity of the more refrangible rays.

The other external conditions which must be considered in heliotropic investigations are so simple that they do not call for any special explanations. Where they are of importance they will be self-evident.

It is very essential, however, to realize that the heliotropism of an animal often manifests itself clearly only during a definite, often decisive, period of its existence, only to diminish again or to disappear entively later. It was only by observing for weeks and months the animals described in this treatise, which for the most part I raised myself, that I have been able to establish this fact.

The caterpillars of Porthesia chrysorrhœa, for example, are energetically positively heliotropic only during a certain period of their existence, when they have just left the coccoon in which they have wintered, and have not yet taken food. At this time the entire existence of these animals is a function of the light. Under natural conditions they hatch out on a warm spring day. The light compels them to creep to the tips of the branches, where they find their first nourishment in the young buds. When fed they are still positively heliotropic, but very much less so than before. If anyone should examine them in this condition, he would scarcely pronounce them heliotropic.

It is not, however, a certain date of the year which governs this heliotropism; for whenever I forced the animals to leave their nest (by raising the temperature), whether at the beginning of summer or of winter, they were indefatigable in their attempts at creeping toward the source of light.

Winged ants are pronouncedly dependent on light only at a definite period of their existence - at the time of their nuptial flight. The same animals which were actively helio- 
tropic at the time of the nuptial flight were practically indifferent toward the light a few days previously. In the same way, later on their heliotropism was entirely pushed aside again by another form of irritability, frequently encountered in the animal kingdom, and to which I shall soon return.

Fly larvæ also possess very different forms of heliotropic irritability at different epochs in their existence. Negative heliotropism is not very distinct in the newly hatched larvæ; but the animals turn their ventral surfaces toward a sufficiently intensive source of light without otherwise being influenced by the direction of the rays of light. Full-grown larvæ, however, place their median planes very sharply in the direction of the rays of light, provided the light is sufficiently intense. I believe that this periodic appearance of heliotropic irritability plays a great rôle in the ecology of animals. The periodic migrations of many animals, such as birds of passage, might be explained in this way.

It is a well-known fact that the irritability of an animal in the larval stage may be entirely opposite in kind to that of the adult stage. This phenomenon is very common. The larva of the fly is negatively heliotropic, while the imago is positively heliotropic; this is also the case with June-bugs and many other animals. I encountered this inversion of the sense of heliotropism when the animal changed from the larval stage to the mature state so frequently that for a time I thought it a universal rule. Such, however, is not the case. Caterpillars, for example, behave toward light as does the imago, as I know from my own experience and from what I can find on the subject in the literature.

The behavior of an animal is determined by the sum of all the forms of its irritability. The heliotropic irritability, therefore, may be obscured by a more powerful irritability of another sort. This is often due to a special kind 
of contact-irritability, which, so far as I know, has not yet been recognized. Many insects are compelled to bring their bodies in contact with the surfaces of solid bodies in a very definite way. My attention was called to this phenomenon in my experiments on animal geotropism, in which I allowed the animals to move about on geometrically simple bodies bounded by plane surfaces. I noticed that the animals rarely remained on the plane surfaces, but collected about the edges, particularly the vertical ones. It is worthy of note that certain animals always seek the concavity of the angle between the sides of hollow cubes, while others just as constantly move on the convex side. The caterpillar of Porthesia chrysorrhœa is an example of the latter type. The other form of this contact-irritability, which leads the animals to the concavity of the angles, is very common. The following observations show how this form of irritability might easily be confused with the irritability toward light, and so lead to a misconception of the behavior of the animal toward light.

I studied for several weeks a large number of moths of the species Amphipyra. The animals are remarkable in that they are more given to running than to flying. The rapidity of their running movements calls to mind the lively movements of cockroaches and ants. While formerly I had found that all butterflies are positively heliotropic, I observed that Amphipyræ when let loose, did not fly to the window, but to the nearest wall or to the floor, where they ran about nimbly and crept under the first suitable object, like cockroaches. This looked as though the animals fled from the source of light. Yet it could be shown that the animals move toward a source of light, and that the inclination to creep intocrevices depends upon the contact-irritability, which was mentioned before. The following experiments always succeeded: In the evening, when a lamp was brought into 
the neighborhood of a box containing the animals, those which reacted at all always flew with great violence to the side of the vessel which was turned toward the light. In no case did they fly in the opposite direction. The experiment was unequivocal and could be interpreted in but one way. So far as the contact-irritability is concerned, the animals collected in the four concave vertical edges when kept in a cubical wooden box, which was covered on top with window glass. In this position they assumed an indifferent orientation toward the source of light. To make perfectly sure of this fact, I employed the following method: I placed a plate of window glass so close to and parallel with the plane of the floor of the vessel containing the animals that they could just wedge themselves in between the floor and the window glass. The glass plate was entirely exposed to the light. Those animals which by chance came to the edge of the glass plate crept under it, and remained in this position exposed to the light, in contact, however, both above and below with solid bodies. On the next day all the animals were under the glass plate. The animals are therefore forced to bring their bodies in contact with other solid bodies, and it is this (and not the light) which causes them to creep under solid bodies. I placed a ball of paper in the vessel containing the animals; a part of them crept under the paper and a part into its folds. In nature these butterflies remain in the clefts on the bark of trees or on the ground in meadows.

Forficula auricularia are found in great numbers in vertical crevices (such, e. g., as the spaces between gate and gatepost, in the entrance to gardens). I obtained the animals for my experiments by hanging a cloth of cotton on the top of a small grape vine. The animals collected in the folds of the cloth. These animals in reality move away from the light; that is to say, they are negatively heliotropic; but it 
would be wrong to attribute their tendency to creep into the folds of the cloth to their negative heliotropism. When I experimented on these animals with the glass plate, I found that they wedged themselves under it, and remained there exposed to broad daylight, rather than creep away from it. Inside of a box the animals collected in the concave edges; and it was very noticeable that the animals rarely ran over the free surfaces, but nearly always along the edges, as if it were ever necessary for them to have their sides in contact with solid bodies.

I believe that this form of contact-irritability is identical with the important phenomenon, observed by J. Dewitz, that spermatozoa are compelled to turn a certain side of their bodies toward solid bodies. Because of this contact-irritability a spermatozoon is never able to leave a cover-glass or a glass slide when once it comes in contact with it. I have observed the same phenomena in hypotrichal Infusoria. These always turn one side of their bodies, the ventral, toward solid bodies. They further resemble the spermatozoa observed by Dewitz in that they alter the direction of their movement always in the same sense, so that on the coverglass of a microscopical preparation are found only Infusoria which move in one direction, while on the glass slide they seem to move in the opposite direction.

In order to distinguish this form of contact-irritability from other forms of contact-irritability (such as the rolling up or progressive or retrogressive movements when touched), I shall call the peculiarity, possessed by some animals, of orienting their bodies in a definite way toward the surface of other solid bodies, stereotropism.

The co-operation of other forms of animal irritability with heliotropism is so simple as to be self-explanatory wherever we may encounter it in our experiments.

1 J. Dewitz, Pflügers Archiv, Vol. XXXVIII (1886). 
V. THE POSITIVE HELIOTROPISM OF THE CATERPILLARS OF PORTHESIA CHRYSORRHEA

I will enumerate the observations which show the identity of animal and plant heliotropism in the caterpillars of Porthesia chrysorrhœa. I shall mention only such experiments as in my experience were always successful under the given conditions, and which may be taken as the prototype of the experiments made upon all the animals treated of in this discussion.

1. The direction of the progressive movement in animals is determined by the direction of the rays of light. - I placed a large number-about a hundred specimens of the small gregarious caterpillars of Porthesia chrysorrhoea which had just crept out of the web in which they had passed the winter-into a test-tube. They had not fed as yet, and in this hungry condition they were exposed to the light. The temperature of the room was necessarily more than $12^{\circ}-15^{\circ} \mathrm{C}$, as otherwise they would have crowded together and fallen asleep again - a state in which they react neither to light nor to gravity.

Experiment 1. - If the test-tube is laid on a dark table, so that the longitudinal axis of the tube is perpendicular to the plane of the window, the animals, which are at first scattered about irregularly, all assume the same orientation. They creep to the upper portion of the test-tube, turn their heads toward the window, and with their ventral surfaces and their heads turned toward the light creep in a straight line toward the window side of the test-tube. The process requires from one to five minutes, according to the temperature and the condition of the hibernated animals. All without exception, provided they are not sickly, move in the direction of the rays of light to the window side of the testtube. If the tube is turned about an angle of $180^{\circ}$, the 
process is repeated, the animals creeping to the window side of the glass just as before. If, however, the position of the glass remains unchanged, the animals remain permanently crowded together on the window side of the test-tube.

Experiment 2. - If the test-tube is laid on the table with the longitudinal axis parallel to the plane of the window, the animals $F$ gradually scatter uniformly over the whole of the upper part of the tube. The lower portion of the vessel is in consequence again free from animals. If the longitudinal axis of the testtube lies at even a slight angle with the plane of the window, the animals move to the end of the tube nearest the window, and remain there in their customary position.

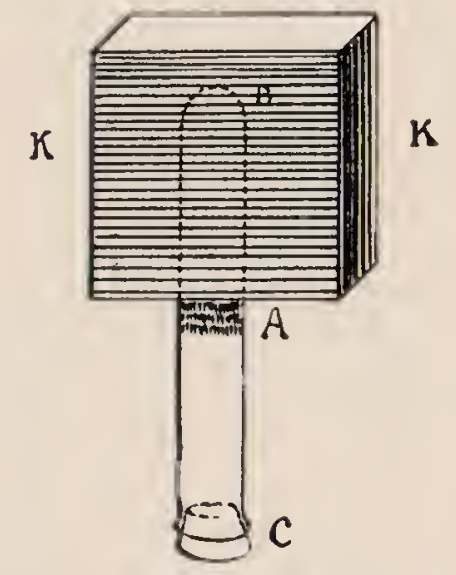

FIG. 1

Experiment 3.- The test-tube is placed perpendicular to the plane $F$ of the window, and at the beginning of the experiment the animals are collected at the window side $B$ of the test-tube (Fig. 1). That half of the vessel which lies nearest the window is now covered with an opaque pasteboard box, $K$. The following then occurs: The animals soon appear at $A$ on the room side of the pasteboard box; as soon, however, as they emerge from the box $K$ into $A$, they turn about, direct their heads toward the window, move to the edge of the pasteboard: and remain at the boundary between the covered and the uncovered portions of the tube, at $A$ and especially at the top of the test-tube. The remarkable thing is that they are not distributed evenly over the whole brightly illuminated part of the test-tube. The explanation is as follows: As soon as the animals near the window at $B$ are covered by the pasteboard, the weak rays of light reflected from the walls of the room fall upon them. 
The animals follow the path of these rays and arrive at the uncovered portion of the tube. As soon, however, as the strong rays of diffuse light fall upon them at $A$, they turn about and direct their heads toward the window, until they come again under the pasteboard which shuts out the diffuse light. They are then again attracted by the light of the room, and so on, until they come to rest at the boundary between the two regions at $A$.

At the beginning of the experiment, before the animals stop moving it can really be seen that they are driven around in a narrow circle.

If at the beginning of the experiment the animals are collected, not on the window side, but on the room side of the test-tube at $C$, they move toward the window until they reach the pasteboard at $A$. If the tube is pulled away from the window for some distance, while the pasteboard remains stationary, the animals begin to move, until they reach the edge of the pasteboard.

If the tube is placed horizontally with the longitudinal axis parallel to the window, the animals distribute themselves over the whole length of that portion of the tube which is not covered by the pasteboard, collecting, however, always on the window side of the tube.

According to the prevailing views of zoölogists and animal physiologists, the movement of caterpillars toward the light is determined by the animals" "fondness for light." They, therefore, move from a region of less intense light to one of greater intensity. That the essential feature, however, is the direction of the rays, and not a difference in their intensity, ${ }^{1}$ is evident from the following experiments.

Experiment 4.-The animals are in a glass cylinder $a$, some $3 \mathrm{~cm}$. in diameter. Light can enter it from all sides (Fig. 2). The inside of a second test-tube $b$, which has the

1 In different parts of the tube. [1903] 
same diameter, is covered with dull black paper, except for a strip about $2 \mathrm{~mm}$. wide. The two test-tubes are placed together on a table so that their longitudinal axes lie perpendicular to the plane $F$ of the window, and the transparent side $c d$ of the glass $b$ is turned up; the animals move along the illuminated side $c d$ from $a$ to $b$, without stopping at the boundary between them, until they reach the window side $c$ of the cylinder. The total amount of light which strikes a caterpillar in the glass $b$, however, is less than in the glass $a$, since all lateral rays are cut off in the former and the animal is struck by rays of light only on its ventral side; in testtube a light falls upon the animals from all sides, though the rays from above and

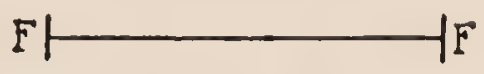
in front are of course the most intense. The animals therefore move toward the source of light in the direction of the rays of light, even if by so doing-to judge from human sensations-they are led from a "bright" to a "dark" place.

In such an experiment no animals are found, as a rule, scattered over the rest of the surface of the glass $b$. If both glasses are turned around so that $a$ is nearest the window side, the animals of course again move from $b$ to $a$.

The experiments described here were carried on in diffuse daylight. In sunlight, however, the results are the same as in diffuse daylight. When the glass is placed with the longitudinal axis in the direction of the rays, the animals move in the direction of the rays toward the sun and collect at the end of the glass which is turned toward the sun, even though in their hungry state they cannot bear the high temperature. When the test-tube is placed with the longitudinal axis perpendicular to the rays, the animals scatter over the 
whole length of the tube, remaining, however, upon its sunny side. Orientation takes place more quickly in direct sunlight than in diffuse daylight.

Experiment 5.-A small pencil SS of direct sunlight is allowed to fall on a table obliquely to the plane of the window through the window F (Fig.

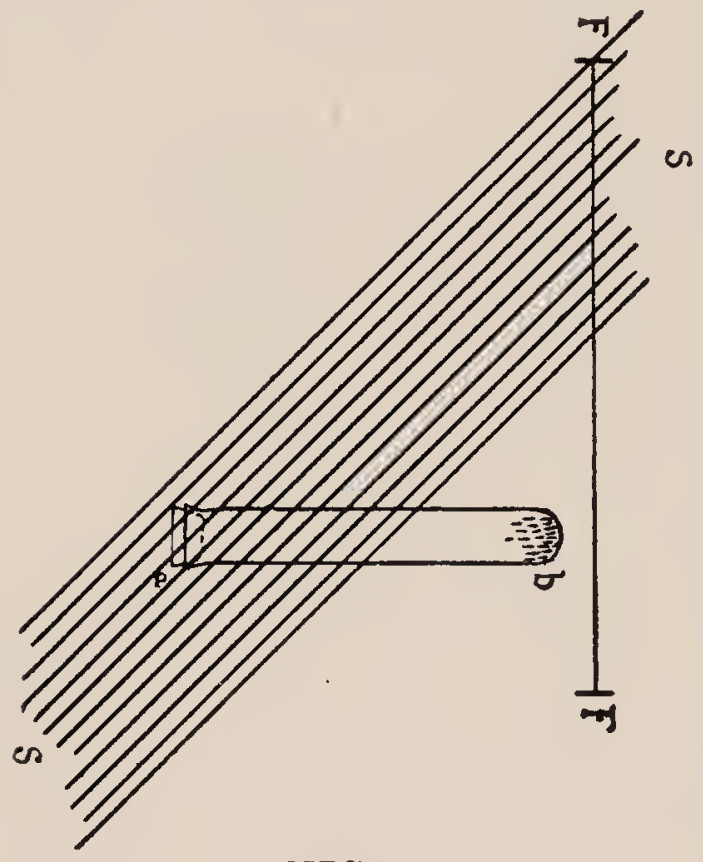

FIG. 3 3). Rays of diffuse daylight fall upon the remaining portions of the table. If at the beginning of this experiment all the animals are at the end $a$ of the test-tube - which is so placed on the table that $a$ is in direct sunlight, while the other half $b$ is in diffuse daylight, and is nearer to the plane of the window than $a$-the following occurs:

The animals move from $a$ through the pencil of direct sunlight into $b$, which lies in the diffuse daylight, where they remain at the cup of the test-tube. They pass from the direct sunlight into diffuse daylight without even attempting to return into the sunlight.

This experiment can be explained only by the assumption that the orientation of the animals is determined by the direction of the rays. The animal can and must follow the rays of diffuse light which have the direction $b \rightarrow a$. If, as is customary with zoölogists, we believed that these animals love the light-or, more correctly, that they prefer the more intense light - it would be impossible to see why they do not remain in the direct sunlight, or at least why they do not hesitate to go into the diffuse light.

From what has been said, no one, I believe, will doubt that the direction of the progressive movements of the cater. 
pillars of Porthesia chrysorrhea is determined by the divection of the rays of light, and not by differences in the intensity of the light in different parts of space. Positively heliotropic animals are compelled to turn their oral pole toward the source of light and to move in the direction of the rays toward this source.

2. The dependence of orientation on the refrangibility of the rays. - I shall now show that it is the more refrangible rays of the visible spectrum which are chiefly concerned in bringing about the orientation of the caterpillars of Porthesia chrysorrhoea.

Experiment 1.-If we place the test-tube on a table and cover it with a box of dark-blue glass, the animals behave as if the vessel were uncovered. Without exception, they move in a straight line to the window side of the vessel and remain there. If instead of blue glass we use red, which to our eyes seems much brighter than blue glass, no change occurs in the orientation of the animals at first; after a long time, however, the animals collect under the red glass on the window side of the vessel. In direct sunlight, however, orientation takes place more quickly. Exactly the same phenomena are observed if an ammoniacal solution of copper is substituted for the blue glass, or a solution of potassium bichromate for the ruby glass. This is also true in the following experiments, where I may not always call special attention to it. This experiment shows (1) that the more refrangible rays have the same effect as mixed rays, and (2) that the less refrangible rays bring about movements in the same way as the more refrangible ones, only their elfect is less intense. The experiment also proves that it is wrong to say, as do the anthropomorphists, that the animals "are fond of" blue and "hate" red; for, were this true, the animals should have been forced to move to the room side of the test-tube when under the red glass, yet they moved 
toward the window. The animals neither "are fond of" blue nor "hate" red, but they are like plants, simply positively heliotropic, and the blue rays are more effective heliotropically than the red. There is, as I shall state here once for all, no difference in direction between the movements called forth by blue light and red light; there is only a difference in the velocity and precision with which these heliotropic movements take place.

Experiment 2.-The longitudinal axis of the test-tube is again perpendicular to the plane of the window. The small caterpillars are at the beginning of the experiment on the room side of the tube. The window half of the test-tube is covered with dark-blue glass. The experiment goes on as if the tube were uncovered; the animals move to the window side of the test-tube, where they remain under the blue cover. If the same experiment is repeated, only so that the blue cover is placed over the room side of the test-tube, the animals again move to the window, where they remain. The experiment proves that the more refrangible rays alone have the same effect as mixed light; and the fact that the animals leave the uncovered portions of the test-tube to creep under the dark-blue cover corroborates what has already been said, that positively heliotropic animals move in the direction of the rays of light even when in so doing they pass from a place of greater intensity of light to one of less intensity.

Experiment 3.-The test-tube again lies horizontally, with its longitudinal axis perpendicular to the window. At the beginning of the experiment the animals are on the window side of the test-tube. If the window half of the tube is covered with red glass (which may seem much brighter to us than the blue glass of the previous experiment), immediately after the red glass has been placed over the animals they appear on the room side of it, and collect at the boundary between the covered and uncovered parts of 
the tube. If at the beginning of the experiment the animals were on the room side of the test-tube, they move until they reach this boundary.

We therefore get the same results by using red glass that we got by using opaque pasteboard in a previous experiment. Taken together with the preceding ones, this experiment proves that pre-eminently the more refrangible rays of mixed daylight are heliotropically effective. Although, as we have just seen, the rays passing through red glass or a red solution are not absolutely ineffective, yet the weak light which is reflected from the walls of the room, and which contains some blue rays, is more effective than the diffused light reflected from the sky after it is filtered through red glass. It is for this reason that the animals on the window side under the red cover migrate to the boundary of the red screen where they are held by the rays of diffuse daylight.

Experiment 4.-If, as before, we place the test-tube with the longitudinal axis perpendicular to the window, and cover it with red glass on the window side and with blue glass on the room side, the animals collect under the blue glass at its boundary with the red glass.

Experiment 5.- - If we place the test-tube with its longitudinal axis parallel to the window, the animals scatter over the whole length of that part of the tube which is covered by blue glass.

From all these experiments it follows that it is chicfly the more refrangible rays which determine the orientation of the caterpillars of Porthesia chrysorrhoea toward light.

The only difference between the heliotropism of these animals and the heliotropism of plants is this, that the less refrangible rays are not so completely ineffective in the case of the caterpillars of Porthesia chrysorrtioea as they apparently are in many plants. This point must, however, be studied more accurately with the aid of a spectrum. 
3. The dependence of the orientation on the intensity of the rays of light.-It is a peculiarity of all animal as well as plant structures that only external stimuli of a certain intensity can call forth reactions. It can easily be shown that at the approach of twilight there comes a time when the rays of diffuse daylight coming through a window no longer attract caterpillars of Porthesia chrysorrhœea.

If the animals are between two sources of light of different intensities, that having the greater intensity is the more effective. This can easily be shown by bringing the animals into a room into which light enters from opposite directions. Other conditions being the same, the animals move to the window nearest them. A maximum limit for the intensity of the light cannot be established, as direct sunlight is in itself effective. Artificial sources of light above a certain intensity and containing the more refrangible rays affect the animals in the same manner as the natural sources of light. In a dark room caterpillars are attracted by a kerosene flame as markedly as moths; the caterpillars, however, are not burned, because they move so slowly that they have time to turn back before the zone of fatal temperature is reached. Such animals as are attracted by direct sunlight may also be attracted by the candle flame, exactly as is the case in positively heliotropic plants.

4. At a constant intensity light acts as a continuous source of stimulation. - If the test-tube which is placed with its longitudinal axis perpendicular to the window is left undisturbed, the animals remain permanently on the side nearest the window. Under these conditions we can also safely open the room side of the vessel without a single animal changing its position or escaping from its cage. It is remarkable, however, that when the test-tube has been left undisturbed all day, the animals keep their position during the night. In this way I have kept animals for several days 
in a test-tube open on the room side; but when I turned the vessel through an angle of $180^{\circ}$ in the daytime, hardly two minutes elapsed before all the animals had moved to the open end of the vessel which was now turned toward the window. Under these conditions they of course escaped from the test-tube. A position which the animals have assumed under the influence of light is usually not changed. when the light is removed, unless some other stimulus comes into play.

5. On negative geotropism and contact-irritability in the caterpillars of Porthesia chrysorrhoe.-The reader may perhaps have noticed that in all of these experiments on caterpillar's the test-tubes were always placed with their longitudinal axes horizontal. This was due to the fact that the animals behave like plant structures, not only in regard to their heliotropic, but also in regard to their geotropic, irritability. Just as is frequently the case in positively heliotropic plants, we find that the caterpillars are also negatively geotropic; that is, they are compelled by gravity to creep vertically upward until they come to rest in the highest part of the test-tube. These experiments were made in a dark room, with the long axis of the test-tube in a vertical direction. If the test-tube is inverted, the animals again creep to the top; if left undisturbed, the animals remain in the uppermost regions of the test-tube. It is necessary in these experiments, as in those on heliotropism, to have the temperature of the room at least $15^{\circ}$, preferably as high as $20-22^{\circ}$. It is simplest to put the test-tube in one's pocket with its longitudinal axis vertical. In a few minutes the animals are found at the highest point in the tube. An increase in temperature increases the geotropic irritability of the animals.

It must now seem questionable whether in our former discussion of the heliotropism of these animals we were 
justified in taking as the effect of light the movements of the animals to the top of the test-tube; it might, indeed, be a geotropic phenomenon. To decide this the animals were placed in a test-tube which was lined with thick black paper except for a strip $2 \mathrm{~mm}$. wide. The uncorered strip was turned downward, so that light could enter the vessel only from below. Diffuse daylight was reflected through the slit from below by means of a mirror. The animals collected in the lower, lighted portion of the glass vessel. Their heliotropism is therefore more powerful than their geotropism, even when only weak diffuse daylight is used.

The geotropic experiments succeed only when the animals have been in the light for some time and have not yet come to rest. When the animals are kept in the dark for a long time and the test-tube is not disturbed, they do not creep upward. The orienting effect of the light always exceeds that of gravity. The effects of gravity, like the effects of light, usually appear only during certain periods in the life of the animals; at any rate, they cannot always be demonstrated with certainty.

The contact-irritability of the caterpillars of Porthesia chrysorrhœa shows itself by the way in which the animals remain in the corners and convex sides of solid bodies. I covered the boxes in which I cultivated my caterpillars with large, square glass plates. These did not close the box tightly, so that the animals could creep out and creep upon the glass plates. Only rarely, however, were they found on the free surface of the plates. The animals moved along the rough edges of the plate until they reached the window side of the dish. I confirmed this observation almost daily for months. When I placed the animals upon the outside of a cubical block, they collected by hundreds in one of the upper corners. Of course, only a few have room in the corner itself, but, as is generally the case with these ani- 
mals, when a few have collected in a spot the others on arriving hold fast to the sides of those already there. An animal at rest acts upon a creeping one as a convex edge. On the other hand, I have never observed that the animals within the cubical box collect on concave edges. From this it follows that the friction of gliding over the convex corners is the source of the stimulation which compels the animal to come to rest there; in moving over the concave corners this friction, of course, does not take place.

These three forms of irritability control mainly the daily life of the animals. We find them in great numbers in fruit trees and bushes, where they pass the winter in their nests; as soon as the warm weather comes, they leave their nests. Positive heliotropism and negative geotropism compel them to creep upward to the tips of branches, and contactirritability holds them fast on the small buds. We can easily show that neither smell nor a special mystical "instinct" leads the animals to the buds, as we are able to compel them by the aid of light to starve in close proximity to food. The animals move to the window side or to the top of a test-tube in which they are kept. If then a branch covered with buds is pushed into the test-tube on the room side, the animals nevertheless remain where light and gravitation have compelled them to go and are holding them. If, however, they once are on the buds, the latter act as a stimulus which may be even stronger than the light. It is in such a case impossible to draw the animals away from the food by means of light.

All these forms of irritability can best be demonstrated on animals which have just left the nest in which they have spent the winter, and which have not yet eaten anything. As soon as they have eaten and are about to moult, their irritability decreases, and at the time of moulting it is almost impossible to show any effect of light or gravity upon them. 
6. The effect of temperature on the caterpillars of Porthesia chrysorrhoca.-The caterpillars of Porthesia chrysorrhœa behave toward a source of heat in a manner opposite to that in which they behave toward light; they move away from the source of heat. If the animals contained in an opaque vessel are brought in the neighborhood of a hot stove, they leave the side of the vessel which is nearest the stove. Yet the heat does not compel the animals to move in a straight line, as they do when struck by the more refrangible rays of light. This directing effect of the more refrangible rays of the visible spectrum is greater than that of the dark heat rays. In this way it is possible for the same animal which flees from the source of the dark rays of heat nevertheless to move in the direction of the sun's rays to the sunny side of a vessel.

It is a well-known fact that irritability in a tissue is a function of the temperature. I have already mentioned that at a temperature of less than $13^{\circ} \mathrm{C}$. the animals are no longer affected by light. It can be shown that heliotropic irritability increases with an increase in temperature. If the animals are kept during the day in a room having a temperature of about $18^{\circ}$, it is found that they no longer respond to light when beyond a certain distance from the window. If, however, the temperature of the test-tube is increased a few degrees, the animals move the more quickly to the window side of the tube the higher the temperature. It can easily be demonstrated that the orientation takes place more rapidly, and that the direction of the progressive movements coincides more nearly with the direction of the rays of light, whenever the temperature is raised. If, however, the temperature is increased to $30^{\circ}$ or over, the animals become very restless; they raise the anterior ends of their bodies higher than is usual in their movement, and so decrease the velocity of their progressive movements. The most suitable tem- 
perature for demonstrating their heliotropic activity lies between $20^{\circ}$ and $30^{\circ}$.

The experiments on the caterpillars of Porthesia chrysorrhœa are typical. I have repeated them on some hundred species of insects, but I have never found a positively heliotropic insect whose dependence upon light was of a different kind from that found in Chrysorrhœea. This fact has given me the impression that all animal protoplasm, as perhaps all plant protoplasm, is heliotropically irritable, and that where this is apparently not the case the heliotropic reaction is inhibited, either temporarily or permanently, by other causes. For this reason it would be useless to publish here every single experiment I have made. This would result in repeating each time the same phenomena, only under the name of a different insect. Since there are only negatively and positively heliotropic animals, it would be of secondary interest to know to which of the two classes the individual animals belong. But I believe it necessary to show by concrete examples what part heliotropism plays in the habits and ecology of animals.

\section{THE POSITIVE HELIOTROPISM AND THE SLEEP OF BUTTERFLIES}

Our knowledge of the behavior of butterflies toward light has, on the whole, remained at that point which is marked by the statement of Réaumur that "it is a singular fact that those butterflies which shun the daylight are precisely those which fly into lighted chambers." The paradox has not yet been explained why those butterflies which are not to be seen by day fly into the flame at night, while the day butterflies apparently do not possess the tragic "instinct" of the night Lepidoptera. There is no lack of conjecture on this point. Romanes believes that the lamp is a "strange object" to the moths, and that "the desire to examine this 
strange object" drives the moths into the flame. We find, however, that the caterpillars of Porthesia chrysorrhœa creep as well toward the sun as toward a lamp. Yet, according to Romanes, the sun ought to be a familiar object to these animals. Such anthropomorphic opinions as those of Romanes are evidently as useless in the analysis of life-phenomena as the speculations of metaphysicians e.g., Hegel's - on physical phenomena. A scientific analysis of the behavior of moths toward light leads to a very simple explanation of the paradox.

Experiment 1.-Specimens of Sphinx euphorbiæ, Bombyx lanestris, and other moths are kept in a large glass box. The box is placed in a room into which only daylight and no artificial light enters. As soon as the animals begin to fly, at the approach of twilight or later, they collect at the window side of their boxes. Whenever the box is reversed the animals fly back to the window side. This experiment is rendered more complete by the following observations:

I kept the pupæ of moths in an open box. Most of the moths hatched at night. On the following morning I always found them collected at the closed window of the room. Here they remained all day exposed to the light. Finally, when I caused the moths to fly by day, I noticed that they flew to the window as do all other positively heliotropic insects. These experiments show that the animals are attracted, not only by a lamp, but also by diffuse daylight. They also show that Réaumur's idea that moths shun daylight is wrong. The experiments indicate that the animals are positively heliotropic toward diffuse daylight, although, as we shall soon see, this positive heliotropism may during the daytime be obscured by another form of irritability.

Experiment 2.-I brought some specimens of Sphinx euphorbiæ into a room which had a window only on one 
side. On the wall of the room opposite the window I placed a kerosene lamp. At the approach of twilight, when the animals began to fly about, I brought them into the middle of the room, so that they were equidistant from the lamp and the window, and left them alone. They flew to the window. Yet, when I brought them into the immediate neighborhood (within about a meter) of the lamp, they flew into the flame. I repeated this experiment and convinced myself that they always flew to one of the two sources of light, either the window or the lamp; to the latter, however, only when they were in its immediate neighborhood.

This experiment shows that the animals do not even prefer artificial to the natural light, but that the artificial light attracts them only when its intensity is greater than that of the diffuse daylight, which is the case at night when the animals are within a certain distance of the lamp, varying with the intensity of the flame. The heliotropic sphere of attraction of an electric arc light is therefore larger than that of a candle flame, and the number of moths attracted by it correspondingly greater.

Experiment 3.- It must yet be proved that it is chiefly only the more refrangible rays of light which determine the movements of the moths. I studied the behavior of Sphinx euphorbiæ, which began to fly at about 9 o'clock in the evening.

The animals were contained in a large box, $40 \mathrm{~cm}$. long, the upper wall of which was of glass. Whenever I turned the box the animals at once flew to the window side and crowded against the upper glass wall through which the light came. When I placed a red glass over the window side of the box, the animals at once flew to the room side. They collected at the edge of the red glass, but on the room side of it, where they were not covered by it. Here they attempted to fly upward. When I used blue glass instead of 
red, they flew under it to the window side of the box. At fifteen minutes past 9 o'clock they came to rest and no longer reacted to light. When exposed to daylight on the following day, they did not stir, and made no attempt to creep away from the light, although sufficient opportunity was offered.

I repeatedly established the fact that the movements of night butterflies are determined by the more refrangible rays of the spectrum on other specimens of Sphinx euphorbiæ. It was therefore not to be expected that in lamplight any other than the more refrangible rays would bring about movements. I have convinced myself that the moths of Geometra piniaria are readily attracted by the light of a lamp when behind blue glass, but not when behind red glass.

The night butterflies, therefore, shun neither diffuse nor intense light, nor do they prefer artificial light to diffuse daylight; the correct expression of the facts is rather this, that most species react to light only at night, when they are positively heliotropic like the day Lepidoptera. We find in butterflies periodic variation in irritability (as in many plants), and these variations correspond to the changes of day and night. As certain flowers open their calices only by night, while others open theirs by day, so certain butterflies fly only by day, while others fly only by night. Both classes of butterflies, however, are positively heliotropic; and it seems as if the irritability of the night butterflies toward light is not less, but even greater, than that of the day butterflies; for the intensity of the light which causes heliotropic phenomena in moths is apparently much less than the minimal intensity which stimulates day butterflies to heliotropic movements.

The phenomena of sleep in butterflies are perhaps more complex than the corresponding phenomena in plants. One thing is, however, certain - that the periodicity of the nocturnal movements of butterflies does not change during the 
first two or three days if the animals are kept in the dark. Under these circumstances the moths become restless at the usual time. Réaumur showed that moths begin to fly in the evening when kept in a box. I must leave it undecided for the present whether this periodicity finally disappears if the animals are kept still longer in the dark. I have tried repeatedly to cause Sphinx euphorbiæ to fly in the daytime by a sudden diminution in the intensity of the light. When I protected the animals from all jarring $I$ never succeeded between 6 and 12 o'clock in the morning. Yet I was easily successful in the afternoon, long before the beginning of twilight. I will cite here several of my experiments. One morning I placed a Sphinx euphorbiæ, which had begun to fly at 9 o'clock on the previous evening, on the window curtain, where it remained quietly. At 2:45 I returned it to its glass box, which stood in a dark corner and into which light fell only through a narrow slit. An hour went by, but the animal did not leave its place. It then moved to the light side of the box, without flying. I carried the animal back to the window, where it remained quietly. After twenty minutes I returned it again to the dark box. Half an hour later, at half-past 4 , it finally began to fly.

The next day $I$ allowed it to remain at rest near the window, and it did not begin to fly until 9 P. .r. at well-advanced twilight. On the following day I kept it in the dark box, and at half-past 3 in the afternoon it had already begun to fly. At noon on the succeeding day a heavy storm came up and it grew quite dark. The moth, which until then had remained quietly at the window, began to fly. I have had the same experience with other examples of this species. These facts seem to indicate that it is possible to influence the time of waking of Sphinx euphorbiæ by diminishing the intensity of the light, but only when they would soon wake up without artificial interference. 
The day butterflies are positively heliotropic like the night butterflies. The only striking feature is that in certain day butterflies the intensity of the light must be very great to bring about heliotropic movements. Specimens of Papilio machaon (which I had raised) remained at rest during the day at a window where they were exposed to the diffuse daylight and could be carried around on the finger; as soon, however, as they were brought into direct sunlight, they flew toward the window in the direction of the rays of light, and this with such force that they dropped down as if stunned. In direct sunlight they pressed themselves closely against the window pane. In diffuse daylight the animals, if they moved at all, crept toward the source of light; but in direct sunlight they flew toward it. My attempts to attract Papilio machaon by the weak light of a kerosene lamp were unsuccessful.

I will add at this point my general observations on the caterpillars of butterflies. I have not found these periodic variations in heliotropic irritability in most caterpillars, not even those of Sphinx euphorbiae. The caterpillars which I studied reacted to light at all times of the day and night. The caterpillars agree, however, with the day and night butterflies in so far as they are all, without exception, positively heliotropic.

This positive heliotropism is most marked in the caterpillar of the willow-borer, which lives in the stems of the willow where it is not at all exposed to light. Such cases are also known in plants. Roots, for instance, are heliotropically irritable, and yet, as Sachs points out, under normal conditions their heliotropism is of no use to them. They can certainly not have acquired it through natural selection. According to the Darwinian theory, we would expect that the caterpillars of willow-borers should be negatively heliotropic, or at least indifferent to light. But the 
behavior of an animal is merely the resultant of all its forms of irritrbility, and so it may happen that an animal is positively heliotropic even when it has no opportunity to make use of it. The larvæ of many saw-flies behave just as the caterpillars of Lepidoptera. I have made observations on the larvæ of Nematus ventricosus, which are exactly like those on Porthesia chrysorrhœe, which have been described.

I have not yet succeeded in demonstrating a heliotropic reaction to diffuse light in the indigenous pupæ. Wilhelm Müller, however, has observed effects of light in South American species. ${ }^{1}$ The pupæ can move at three joints. Only a lateral movement to the right and left is possible in some of the species; in other species only a dorsal movement of the body is possible; in a third species of pupæ a combination of both kinds of movements is possible. Müller observed that all three classes of movements can be brought about under the influence of light. $\mathrm{He}$ found that some pupæ turned not only away from the light, but also toward it. He also found that when the animals had been exposed to the dark for some time, they "needed some time to become susceptible again to the influence of light." In interpreting the phenomena Müller follows the Darwinian idea, so that the thought never occurs to him that he might be dealing with phenomena similar to the heliotropic phenomena of plants.

The negative geotropism of the Lepidoptera.-The movements of very young or recently hatched animals have for the most part been misunderstood, because they have always been considered a function of mysterious "instincts" of the animals, while the direction of their motions is in reality determined by definite external forces. The same cause which prescribes the course of a falling stone or determines the orbits of planets, namely gravitation, determines

1 MULLER, Zoologische Jahrbücher, Vol. I (1886), pp. 568 ff. 
also the path which a butterfly follows that has just emerged from the pupa case. The geotropic irritability is at that time especially strong; the newly hatched animals remain restless, and are compelled to run about until they come to a vertical wall, on which they can put the longitudinal axes of their bodies vertically, with their heads upward. Here they remain quietly until their wings are unfolded. The powerful manifestation of negative geotropism at the time of hatching is no isolated phenomenon in insects. In summer we find great numbers of the ecdyses of the larvæ of Ephemeridæ on the banks of streams. They are found on blades of grass or steep banks, with their longitudinal axes usually vertical and the head upward. That gravity, and not light alone, plays the chief rôle here is shown by the fact that I have found the ecdyses in the same position under bridges where no light could strike them from above.

This observation on the larvæ of Ephemeridæ makes it impossible for us to accept the idea that the "purpose" of the orientation of the freshly hatched imago of a butterfly is that the wings may unfold; for negative geotropism appears in the larvæ of Ephemeridæ at a time when no wings are present. The caterpillars of butterflies are also negatively geotropic like the freshly hatched moths, even though not so markedly. Immediately after hatching geotropism is much stronger in the imago of the butterfly than heliotropism - a phenomenon rarely observed in the animal kingdom. If a freshly hatched imago is on a vertical wall, it does not change its orientation toward the center of gravity even when the direction, refrangibility, or intensity of the light is changed.

What is true of the heliotropism of Lepidoptera, that it is most marked during certain periods of their existence, holds good also for their geotropism. Amphipyra is energetically negatively geotropic immediately after moulting. 
Several days later the animals assume every possible position with reference to the vertical. They prefer to remain on vertical walls, yet they will creep just as readily into horizontal folds and crevices.

\section{THE POSITIVE HELIOTROPISM OF PLANT LICE}

Anyone closely studying a rose covered with wingless plant lice will notice that they are arranged in a definite way on the plant. On a vertical stem they rest with the head downward; on the leaves they are usually found on the underside, mostly on the principal veins. Here one also notices a certain regularity in their orientation, in so far as the animals on the principal vein turn their oral poles toward the stem, and their aboral poles toward the point of the leaf. The orientation of the animals seems therefore to be controlled by the structure of the plant, and not directly by external forces.

But the plant lice do not behave on all plants as on the rose. On a palm, for example, I found no such definite orientation of the animals toward the plant, even though in this case also they show a preference for the lower surfaces of the leaves.

Yet it might seem reasonable to suppose that light or gravity compels the plant lice to seek the lower surfaces of the leaves. I twisted several leaves of Cineraria, the dorsal sides of which were covered with plant lice, so that the dorsal sides were directed upward and toward the window, and fixed the leaves in this position. I watched the animals for two days and found by actual count that the animals remained at rest. I repeated the same experiment on the plant lice of palm leaves, but also with negative results.

My experiments on the orientation of new-born wingless plant lice were practically negative when I removed them from the plant and placed them in a glass vessel. Yet in 
the older wingless animals I could notice an inclination to move toward the source of light. When their wings had sprouted, however, the orientation of the plant lice was extrcoordinarily definite. In this state they are perhaps the most suitable animals we have for demonstrating the phenomena of heliotropism. Not all species are equally irritable; Cineraria afforded me the best specimens. I have never found a species of plant louse which was not definitely positively heliotropic. I kept the plants near a closed window. The animals were attracted by the sun to the window, where they crept upward. When the animals are lightly touched with the point of a pen, they fall down a second or two later. If a glass vessel is held under them, a large number of these animals can be collected in an uninjured condition in a short time. I found it much better to work with such animals as have already flown from the plant, than to collect the winged animals from the plant itself. To obtain the winged plant lice in great numbers it is necessary only to allow a plant which is covered with them to dry out gradually. Under such conditions the wings grow out very rapidly.

All the experiments which were made with Porthesia chrysorrhoea can be repeated with exactly similar results on winged plant lice contained in a test-tube.

As in the heliotropism of caterpillars, the heliotropism of plant lice is determined chiefly by the more refrangible rays, which compel the animals to move in the direction of the rays toward the source of light. If we place the test-tube containing the animals on a horizontal table, they always move toward the source of light, whether this be lamplight, diffuse daylight, or direct sunlight. The orientation occurs the more rapidly the more intense the light. If the intensity of the light is constant, the plant lice, like the caterpillars of Porthesia chrysorrhœea, are compelled to remain perma- 
nently on the side of the test-tube which is turned toward the serrce of light.

If direct sunlight comes through the window, and the tube containing the animals is so placed that one-half lies in the direct sunlight, while the other half is in diffuse daylight, and if the latter half is nearer the plane of the window than the former, the animals will move to the window side of the vessel, like the caterpillar's of Porthesia chrysorrhœa; they leave the direct sunlight and move into diffuse daylight in order to follow as nearly as possible the direction of the rays. The result is the same when the diffuse daylight first passes through dark-blue glass. The animals are compelled to go to the window side of the tube under all conditions, no matter whether the test-tube is covered entirely or only in part by the blue glass, or whether the blue glass is placed over the window or the room side of the tube. The less refrangible rays which pass through deep-red glass are not very effective. In consequence, if the test-tube is entirely covered with red glass, the animals, if not very sensitive, distribute themselves evenly over the whole test-tube, just as in the dark; or, if more sensitive, they collect after a long time on the window side of the test-tube. But even then they do not quite behave as under blue glass. While under blue glass they collect in a very small area on the window side of the tube, under red glass they occupy a much larger area. If only a part of the test-tube is covered with red glass, the animals collect at the window side of the uncovered portion of the test-tube, as the less refrangible rays have only a minimal effect. When I placed a test-tube containing highly sensitive plant lice on a horizontal table perpendicular to the plane of the window, and covered the window side of the test-tube with a bright-red glass, the animals collected at the boundary between the uncovered and the covered part of the 
tube when diffuse daylight entered through the window. The more refrangible rays reflected from the walls of the room were more effective than the rays from the window which had passed through the light-red glass. This prevented the animals from going under the red glass. But when I used direct sunlight, the animals moved under the red glass to the window side of the vessel and remained there. When the animals were collected at the room side of the test-tube lying horizontally on a table and with its longitudinal axis perpendicular to the plane of the window, and an opaque cover was placed over the room side of the tube, while a dark-red glass was placed over the rest of the tube, the animals went under the red glass and gradually collected there on the window side of the tube. But when I placed the opaque cover over the window side and the red glass over the room side of the tube, and the animals were under the opaque cover at the beginning of the experiment, they did not collect under the red glass. The rays reflected from the wall of the room had lost their directing power in filtering through the red glass. The experiments were made in the diffuse light of a dark day. On a bright day the animals moved to the room side of the tube under the red glass.

The rays which pass through red glass have therefore the same effect, only they are weaker than the rays which pass through blue glass.

I have already mentioned the fact that the day Lepidoptera begin to $f l y$ as soon as direct sunlight falls upon them, while in diffuse light their heliotropic movements consist chiefly in creeping. The same difference in the effects of different intensities of light can be easily demonstrated in winged plant lice. In diffuse light of low intensity they move forward by creeping; when brought into the sun they $f y$.

To obtain a measure of the difference in the activity of 
rays of different intensities and refrangibilities, I measured the time it required for the animals in a test-tube to pass a line scratched in the glass, when moving under the influence of light from the room side of the tube to the window. In these experiments I used some sluggish winged plant lice which I had taken from a plum tree. As a rule, the heliotropic movements of plant lice took place much more quickly than in the experiment to be described here. The experiment was made in diffuse light on August 8, 1888. At the beginning of the experiment all of the animals were on the room side.

The animals passed the marks as follows:

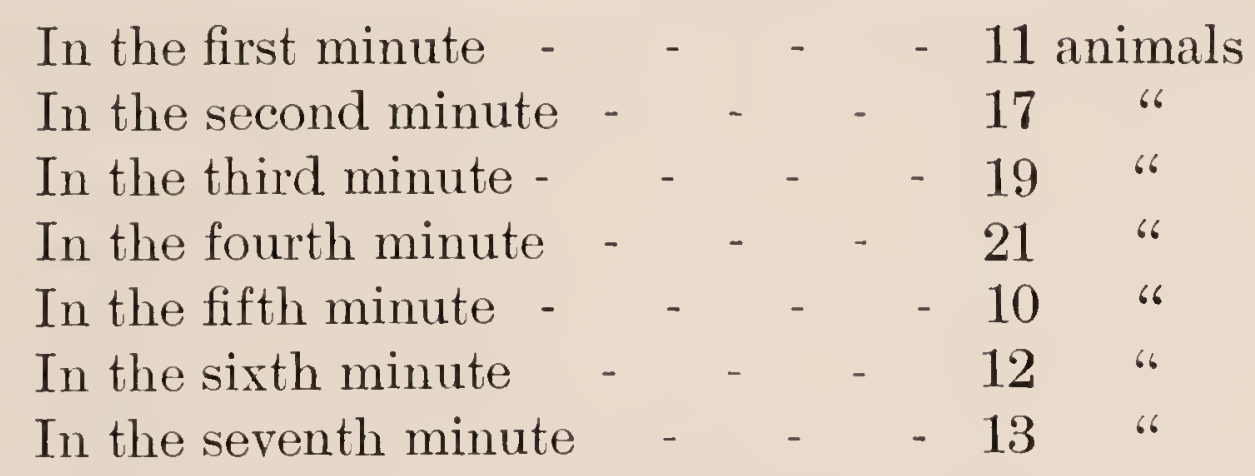

Only three animals had at this time not yet crossed the line. Several minutes later, at 9:20 o'clock, when the sun was coming through a fleecy white cloud, I made the following experiment in direct sunlight with the same animals. At the beginning of the experiment the animals were again on the room side of the tube.

The animals this time passed the mark as follows:

$\begin{array}{llll}\text { In the first minute - } & - & - & 31 \text { animals } \\ \text { In the second minute - } & - & 36 & \text { " } \\ \text { In the third minute - } & - & - & 23\end{array}$

Half a minute later the last sixteen animals had also passed the mark. The velocity of the movement was twice as great in direct sunlight as in ordinary daylight. These experiments were repeated and gave practically the same results. At 10:17 I placed the animals under a dark-blue glass. 
This time it took ten minutes for the animals to orient themselves - a longer time, therefore, than in white light.

At 10:29 I covered the test-tube with red glass, and since I knew that in diffuse light the heliotropic movements take place only very slowly under red glass, I brought the animals at once into direct sunlight. It required seventeen minutes before the majority of the animals had passed to the window side of the mark. In diffuse light it required an hour for orientation to take place under red glass; in a new experiment it required twelve minutes under blue glass.

I noticed no periodic change in irritability in plant lice such as that observed in Lepidoptera, but I did notice a decrease in heliotropic irritability, a kind of rigor when the animals have been left undisturbed in the dark for some time.

If the test-tube remained undisturbed, the animals remained permanently on the side nearest the window. When I very carefully turned the test-tube through an angle of $180^{\circ}$ in the daytime, the animals again moved toward the window, even when they had been left undisturbed for hours. When, however, I kept the animals quietly in the dark, and after some hours carefully placed the tube near a lamp, the animals did not move from the position which they had maintained through the day. They seemed to be asleep. But when I shook the tube so that the animals began to move, they promptly oriented themselves toward the light as often as I turned the tube around.

I found that winged plant lice are negatively geotropic as well as positively heliotropic, as is the case in the larvæ of Chrysorrhøea. If the animals in the test-tube were very vigorous, a change in the position of the tube with reference to the vertical brought about a change in the orientation of the animals toward the center of the earth; they traveled upward at as small an angle as possible with the vertical, and 
collected at the highest point in the test-tube. This experiment must, of course, be made in a dark room. When the animals are first brought into the dark, the experiment can be repeated many times with exactly the same result; every change in the position of the test-tube with reference to the vertical compels the animals to creep upward and to collect at the highest point in the tube. When, however, the animals were kept permanently in the dark, the reaction ceased soon, and the animals remained motionless, no matter how often the position of the test-tube was reversed. The animals were in a sort of rigor. When they were placed on an inclined or vertical plane, they moved upward. Geotropic orientation occurred as soon as the plane made an angle of $30^{\circ}$ with the horizontal; the geotropic movements were the more certain and precise the nearer the plane approached the vertical. When light fell on the animals at the same time, their orientation was determined by the resultant of the direction of the rays of light and gravitation, in which, however, the light was the stronger force even at a great distance from the window.

The winged animals behave toward a source of heat in the same manner as the caterpillars of Porthesia chrysorrhøea. When I brought the animals in an opaque vessel into a room having a temperature of $18^{\circ}$ and placed them near a stove, they left the side of the vessel which was turned toward the stove, as soon as its temperature increased a few degrees. At a temperature of $9^{\circ}$ the animals were so sluggish that a definite reaction to light or gravity did not take place. A temperature of $20-24^{\circ}$ is the most suitable for the experiments. When I surrounded one-half of the vessel with a water-bag having a temperature of $20^{\circ}$, the other half with one having a temperature of 10.5 the animals moved, under the influence of light, from the warmer into the cooler area. But they did not move far into the latter, as their movements 
soon ceased. Under the influence of light, the animals also moved from a region having a temperature of $12^{\circ}$ to one having a temperature of $24^{\circ}$.

VIII. THE CONNECTION BETWEEN HELIOTROPISM AND SEXUALITY IN ANTS

At the time of sexual maturity the male and female ants fly from the nest on a warm day to pair in the air. This "nuptial flight" is, as shown by the following observations, determined by a very pronounced positive heliotropism, which appears especially at the period of sexual maturity.

I discovered a nest of brown garden ants in the wall of a house which was struck late in the afternoon by direct sunlight. In August, 1888, I observed that on warm days in the afternoon, as soon as the sun struck the wall, at about 5 o'clock, the winged ants came out in swarms and then flew away in the direction of the rays of sunlight. I procured a large number of winged ants from such a swarm and studied their behavior toward light. These animals were energetically positively heliotropic, and behaved in all respects like the caterpillars of Porthesia chrysorrhœa.

When I put the winged ants into a test-tube and placed this with the longitudinal axis perpendicular to the plane of the window, the animals moved to the window side as often as the tube was turned around. The velocity of the heliotropic movements was greater in these animals than in any others that I have studied. When the tube was not disturbed the animals remained on the window side nearest the window. When the longitudinal axis of the test-tube lay parallel to the plane of the window, the animals distributed themselves evenly over the whole length of the tube. When one-half of the tube was in direct sunlight, while the other half was in diffuse daylight, but nearer the window, the animals collected in the window side of the tube, they went from 
the direct sunlight into the shade. The direction of the rays, and not the distribution of the intensity of the light, in the test-tube, therefore, determines the direction of the progressive movements.

The blue rays were pre-eminently effective. When the test-tube was covered with blue glass, either entirely or in part, the orientation was changed in no way. When the tube was entirely covered with red glass, the movements occurred more slowly. The animals finally collected on the window side, but it took a long time. When the tube lay with the longitudinal axis perpendicular to the window, and the portion nearest the window was covered with red glass, the animals collected at the boundary between the uncovered and covered parts. Diffuse daylight affected the animals just like sunlight. These facts may suffice to show that at the time of the nuptial flight the winged ants are energetically positively heliotropic.

Yet I found that up to the time of the nuptial flight, light had practically no effect on winged ants which were taken from the same nest.

Animals which I collected after the nuptial flight also did not react very distinctly to light. If heliotropism was still present at all, it was obscured by other forms of irritability, particularly stereotropism.

The nuptial flight of the ants of this nest always took place at about 5 o'clock in the afternoon, when the sun's rays fell upon the nest. That it was the latter condition, and not the time of day, which determined the period of flight is shown by the fact that in other nests, which were reached by the sunlight earlier in the day, the flights took place earlier. Usually the flight occurs at about noon, when the sun's rays strike the earth perpendicularly and the temperature is relatively high. Both the males and the females which I collected from the swarm which had left the nest 
late in the afternoon escaped through the window on the next day at any time that I freed them. The scent of the females therefore does not determine the nuptial flight of the males, and vice versa; after sunset the ants no longer flew away when liberated.

I have already shown that direct sunlight or intense diffuse daylight calls forth flight movements in plant lice and day Lepidoptera. This also occurs in winged ants. In diffuse daylight the male and female ants move toward the source of light only by using their legs; in direct sunlight, however, they fly.

Sunlight, therefore, causes flight movements in ants at the time of sexual maturity, and this fact determines the nuptial flight. Immediately after copulation another form of irritability becomes more prominent ${ }^{1}$ which compels the ants to to crowd into crevices (to "found a new nest").

The connection between sexuality and heliotropism in ants is shown still further by the fact that at the time of the nuptial flight no heliotropism can be demonstrated in the workers. Workers taken from the same nest as the other ants when placed in a test-tube moved about irregularly in it, and finally came to rest on the stopper, no matter in what position I placed the tube with reference to the window. I then placed several winged ants which reacted energetically toward light in the same tube with the workers. The workers apparently became now also positively heliotropic, that is to say, they moved with the winged ants to the window side of the tube whenever it was reversed. This lasted, however, only some ten minutes, when the workers settled again permanently on the stopper and were no longer affected by the light while the winged ants reacted to the light just as before.

The observations of Lubbock seem to indicate that heliotropism may be present also in the workers at certain periods

1 Stereotropism. [1903] 
in their existence. In the experiments of Lubbock the workers contained in the nest not only collected under red glass, but also carried their larvæ there. The animals are therefore negatively heliotropic. ${ }^{1}$

All these facts, however, do not yet exhaust the connection between sexuality and heliotropic irritability. The heliotropism of the male and female ants is also different, inasmuch as it requires more intense light to cause heliotropic movements in females than in males. In isolating the males and females of the same swarm I noticed that the females had ceased to execute heliotropic movements before it seemed as if twilight had really begun. The males however still collected on the window side of the tube long after sunset. Experiments with colored glasses succeeded in males when the light was so faint that I had difficulty in distinguishing the color of the glasses. On dark, cloudy days females showed no heliotropic reactions toward the window, while the males did. It harmonizes with this observation that on cloudy afternoons I saw occasionally winged males leave the nest, but no females.

As soon as the intensity of the light had become so small that heliotropic phenomena were no longer produced, another form of irritability appeared in the winged ants, especially in the females, namely, stereotropism. The animals then crowded into all crevices. I placed the animals in a dark box, and laid a small, folded piece of velvet into one corner of it. After a few moments they had crept into the folds of the velvet. With the males it took a much longer time than with the females. This irritability, however, did not appear as long as the light was sufficiently intense to call forth heliotropic phenomena. When exposed to light, the animals crept neither under the piece of velvet nor into crevices. It is very probable that a similar difference in heliotropic irri-

1 The observations recorded in Lubbock's paper admit another possibility. [1903] 
tability exists in the two sexes of the Lepidoptera. Réaumur states that in the main only males fly into the candle flame. From this fact, which is correct, it follows that it must require a more intense light to cause the females to execute heliotropic movements than is necessary for the males. Both male and female moths are attracted by sources of light which are stronger than the candle flame, for instance, the electric arc light. It is a well-known fact that the females fly less than the males. It is imaginable that this is due to the fact that the females are less irritable toward the light than the males.

The difference in the irritability of male and female ants toward light brings up the question as to whether the difference in the development of the sense organs, particularly the eyes, which is often observed in males and females of the same species, is connected with this difference in irritability. The males of ants have larger eyes than the females. But the cause of the difference in sensitiveness may lie deeper, as is, for example, indicated by the following observation made by Semper: "In all species of the cave beetle Machærites only the females are blind, while the males have well-developed eyes; notwithstanding this fact they always live together." Eyes therefore develop more easily in males than in females even in the dark. It might be worth while to determine whether in these cave-dwellers the males are also heliotropically more sensitive than the females.

IX. THE NEGATIVE HELIOTROPISM AND OTHER FORMS OF IRRITABILITY OF THE LARVA OF MUSCA VOMITORIA

The phenomena of irritability in negatively heliotropic animals obey the same laws as those in positively heliotropic animals; with this difference, however, that negatively heliotropic animals turn their aboral poles toward the source of light instead of their oral poles, and that in consequence the

1.SEMPER, Die natürlichen Existenzbedingungen der Thiere, Vol. I, p. 101. 
direction of their progressive movements under the influence of the rays of light is away from the source of light. My description of negative heliotropism need therefore be but brief. I have chosen as an example of negatively heliotropic animals the larve of Musca vomitoria, which are additionally interesting in that they are completely blind. Heliotropism in animals is therefore a characteristic of their protoplasm, and not a specific characteristic of their eyes; just as in plants, which have no eyes.

In order to study the negative heliotropism of Musca larvæ it is best to take the almost fully grown larve fresh from the cadaver on which they were reared. When the light, which may be either diffuse daylight or direct sunlight according to the sensitiveness of the animals, is of the proper intensity, the directing influence of the rays of light can be demonstrated more beautifully in the larvæ of the fly than in any other animal. I placed a number of these animals on a horizontal board and exposed them to sunlight. This was at about 4 o'clock in the afternoon, when the rays of light fell obliquely through the window. I shut out that part of the rays which came through the window from above by means of blinds. As soon as the animals came into the sunlight, they were oriented with their oral poles toward the room, and their aboral poles toward the window. They crept with mathematical precision in the direction of the rays. When a shadow was thrown on the board by a penholder, it could be noticed that the animals moved away from the light in a direction exactly parallel to the edge of the shadow. The directing force of the rays was so strong that the animals crept closely along the edge of the shadow without crossing it. They acted as though they were impaled on the ray of light which passed through their median plane. When I turned the board around, the animals immediately turned about also, and again placed 
their median planes in the direction of the rays. (That this was due to the effect of the light, and not a compensatory movement that might have been produced by the rapid turning of the board is shown by the fact that compensatory movements do not exist in Musca larvæ.)

I was able to show that fly larvæ are compelled to move from less intense light into more intense light under the influence of the rays of light, just as it could be shown that positively heliotropic animals do not go from dark places to light ones, but follow the direction of the rays, even when by so doing they move from a region of greater intensity of light to one of less. I put the almost fully grown larvæ into a testtube and placed it horizontally on the table, with its longitudinal axis perpendicular to the plane of the window. The sun's rays made a small angle with the window. By means of a screen I arranged the test-tube so that only diffuse light fell through the window upon the half turned toward the window, while direct sunlight fell upon the half turned toward the room. At the beginning of the experiment the animals were all on the window side of the test-tube. They immediately moved from the shaded part into the direct sunlight on the room side, and remained there.

Incidentally I was able to observe that the light stimuli which strike the oral pole of these completely blind animals are most important in the orientation of the animals toward light. When the animals crossed the boundary from diffuse light into direct sunlight, the reaction caused by the increase in the intensity of the light did not take place until a half or a third of the body of the animal was in the sunlight (because in all phenomena of stimulation some time elapses between the application of the stimulus and the reaction to it). The animal checked its movement and turned its head through an angle of $90-130^{\circ}$ from side to side. If in so doing the head again came into the shade, the animal 
returned into the shade; but if this did not happen, as was more usually the case, the animal continued its movement into the sunlight. The animals did not always check their movements in passing from a shaded area into the sunlight. Often they moved without delay from the shade into the sunlight. The following observation shows that the rays of light which strike the head mainly determine the orientation: When I placed a fully grown animal on a board, and pushed the board from the shade into the sun, so that only the head of the animal was struck by sunlight, the larva immediately placed its median plane in the direction of the sun's rays. When, however, I put only the aboral pole into the sunlight, this orientation did not occur. Animals from which the first few segments of the oral pole had been amputated no longer oriented themselves toward the light. Yet little weight is to be given to vivisection experiments, which are followed only by an inhibition of the effects of a stimulus.

When I allowed the sun's rays to fall on the plane of the board perpendicularly, the animals moved over it in all directions. As in this case the animals could not follow the direction of the rays of light, it had no other influence upon them than to increase their restlessness, and no uniform orientation resulted.

It could be shown very beautifully in these full-grown larvæ that essentially only the more refrangible rays are concerned in exercising a directing influence upon these animals. I placed a large number of fully grown larvæ on the middle of a horizontal board in a darkened room, and exposed them to the sun's rays which made but a small angle with the horizon. Within ten to twenty seconds every animal had placed its median plane in the direction of the rays of light, and moved exactly parallel to the shadow of a vertical object which had been thrown upon the board for comparison. I treated a new lot of animals in exactly the same way, but 
before exposing them to the sunlight I covered them with a box of dark-blue glass. Within ten to twenty seconds these animals had also placed their median planes sharply and precisely in the direction of the rays of light, in which direction they moved toward the room side. When I took a third lot of fresh animals and covered them with red glass, the orientation of the animals into the direction of the rays did not occur. They crept to the right and to the left, occasionally moving a short distance toward the source of light; but even after minutes under the red glass the precise orientation of the animals, which followed under the blue glass in a few seconds, did not occur. Under red glass the animals behaved toward direct sunlight just as they did under blue glass toward very weak daylight. That the rays which pass through red glass are not absolutely without effect seems to be shown by the fact that the animals avoided going to the window side, and that they finally collected at the room side of the board. The directing force of the red rays seems therefore to be limited to this, that the animals will not move for long distances toward the source of light. In consequence, the animals must collect ultimately on the room side of the vessel.

In all the previous experiments the animals were on a plane board. When at the beginning of an experiment the animals were collected on the window side of a test-tube which lay horizontal and perpendicular to the plane of the window, in direct sunlight and under blue glass all the animals turned their oral poles within ten seconds toward the room side of the tube. In about twenty seconds they migrated to the room end of the tube. When the same animals were exposed in the same way to direct sunlight, but under red glass, they neither oriented themselves nor moved toward the room side of the tube during the next four minutes, even though they were very restless. 
In this case, therefore, just as in the case of the positively heliotropic animals, it is chiefly the more refrangible rays which effect the orientation of the animals. The heliotropic influence of the less refrangible rays, however, is much less in the eyeless fly larvæ than in any other animals that I have studied. The animals moved in the direction of the rays, even in diffuse light, at a distance of one meter from the window, but the less the intensity of the light, the more easily did other stimuli (such as contact stimuli) cause a deviation of the animals from the straight line. I have often repeated these experiments in the course of the last two years, and have each time obtained essentially similar results. The irritability of the animal is, however, not always the same; especially does its irritability vary during different periods of its life. I have, however, convinced myself that the larvæ are negatively heliotropic even immediately after being hatched, although they do not move as precisely in the direction of the rays as the fully grown larvæ.

I placed some fly eggs on smoked glass plates and allowed them to hatch. As the larvæ removed the soot in their path, they thus registered graphically the paths they took from the eggs. The glass plates lay on a horizontal table in a room lighted from one side only. The paths followed by the larvæ ran, almost without exception, toward the room side of the plates. In the few exceptions the path usually ran first toward the window, then bent, and went toward the room side of the plate. It was neither a mysterious force of nature nor an obscure "inherited instinct" which dictated the direction of the movements of these animals, but only the direction of the rays of light, just as gravity determines the orientation of the Lepidoptera when they emerge from the chrysalis.

When the diffuse daylight which struck the larvæ came 
from two windows the planes of which were at an angle of $90^{\circ}$ with each other, the paths taken by the larvæ lay diagonally between the two planes. ${ }^{1} \quad$ When I placed the plate with the eggs in an absolutely dark room, the paths followed by the larvæ ran concentrically around the nest; the animals had scattered equally in all directions over the plate, but, contrary to the behavior of the animals in the light, which always moved as far as possible toward the room side, the circle in which the animals moved in the dark was very narrow. They did not leave the glass plate. The constant intensity of the light acts, as in the case of the positively heliotropic animals, as a constant stimulus which causes the animals to move in one definite direction (either toward or away from the source of the light), until some other stimulus intervenes, which modifies or abolishes the effect of the light.

In my preliminary communication on animal heliotropism I mentioned an effect of light on fly larvæ which I called a kind of anisotropy, and which I am at a loss how to include under the other phenomena of heliotropism. The phenomenon under discussion appears only in intense light and in newly hatched or very young larva. The phenomenon consists in this, that the animals ${ }^{2}$ turn their ventral surfaces toward the source of light without placing their median plane in the direction of the rays. I have never seen this orientation in adult larvæ. When I put the animals into a test-tube placed with its longitudinal axis perpendicular to the window, and exposed them to the direct rays of sunlight or diffuse light close to the window, the animals left the lower side of the tube and moved to the upper. In this the animals, therefore, resembled positively heliotropic animals, and I might have believed that I was dealing with one of

1 This experiment was recently published by an American physiologist as a new discovery to prove that I had overlooked the importance of the intensity of light! [1903]

2 When kept in a test-tube. [1903] 
those cases occasionally observed in plants where in light of great intensity the heliotropism of an organ is the opposite of that in light of less intensity. A closer examination, however, showed this not to be the case. When the testtube lay perpendicular to the plane of the window, positively heliotropic animals contained in it moved, as we have seen, not only to the upper, but also to the window side of the testtube. This was not the case with the newly hatched fly larræ. They all turned their ventral surfaces toward the source of light, but otherwise moved about irregularly. I placed the animals in a test-tube which was covered with black paper, except for a small slit, and let direct sunlight enter the tube only through the slit. The animals which were on the lower side of the tube left it as soon as the light struck their backs, and crept upward; but no animal which was sheltered from the light was attracted to the upper, lighted side of the tube, as was the case under similar conditions in the positively heliotropic caterpillars of Chrysorrhœa. When I held the glass vertically, more animals collected on the window side, but they did not all creep upward, as did the positively heliotropic animals. When I placed the animals on the outside of a test-tube, they did not move upward, but collected for the most part on the under side of the tube. This experiment was not very decisive, however, as the animals easily fell off the tube. These facts can be interpreted in no other way than that the intense light compels the fly larva to turn their ventral surfaces toward the source of light, in which condition they are indifferent to the orientation of their median planes toward the rays of light. The ventral position is assumed only when the animals are exposed to light. With this, however, the striking features of the movements of orientation in fly larvæ are by no means exhausted. While the movements of orientation in all the other animals go on 
under blue glass just as rapidly and in the same way as in mixed daylight_-since in mixed daylight it is chiefly the more refrangible rays which are heliotropically effective-the ventral orientation of fly larvæ which has just been described occurs neither under blue nor under red glass. In direct sunlight it took one to one and a half minutes before the animals were densely gathered on the upper side of the horizontally lying test-tube. Not one of these animals moved to the upper side of the tube in less than twenty-five minutes under red glass, or in less than five minutes under blue glass.

The ventral orientation of the Musca larvæ toward a source of light can be observed most distinctly in freshly hatched larvæ. As the animal grows larger, the phenomenon becomes less marked. The lump of eggs laid by a fly was distributed among three tubes. In all three tubes the animals immediately after hatching oriented themselves ventrally toward the diffuse light. I then fed meat to the animals in one tube and left the animals in the other two tubes unfed. On the next day the unfed animals were oriented ventrally toward the daylight, while this was not the case in the rapidly growing larvæ which had been fed. I have obtained the same result by feeding the larvæ of one lot of eggs with fat, while another lot was given lean meat. The latter grew more rapidly than the former. While those fed on fat were oriented ventrally in diffuse daylight, direct sunlight was necessary to bring about this effect in those fed on meat.

I might have doubted that this was the effect of light, had I not been able to prove that with a decrease in the intensity of the light the phenomenon becomes less distinct, and finally disappears entirely. I do not think that the ventral orientation could have been the effect of heat, as the animals move away from a non-luminous source of heat, as 
we shall see presently. Because of the preponderance of this rentral orientation, it is no easy matter to demonstrate the negative heliotropism of the young larvæ in a test-tube; they assume the ventral orientation, and no longer trouble themselves about the direction of the rays of light.

The orientation of the larva of Musca toward a source of hect.-If a Musca larva in its movements comes to a spot where the temperature is only one degree higher than in the surrounding area, it stops and turns its head laterally. If in so doing its head encounters a spot with a lower temperature, it turns thither and continues to move in this direction. One can easily convince oneself of this by laying the tip of a finger on a spot on the outside of a test-tube containing the larræ. The increase of temperature of the spot touched can be ascertained by a sensitive and finely graduated thermometer. As soon as the animal comes to the spot touched by the finger, it turns its head. If it does not turn far enough to touch a cooler spot, it continues in the old direction to the region of higher temperature. According to this, the stimuli which reach the oral pole determine the orientation of the animal toward a source of heat also, just as in the case of light. If the experimenter puts a test-tube containing a large number of Musca larvæ into his pocket, where no light reaches them, the animals collect in a few minutes densely on that side of the tube which is turned away from his body. The same thing happens when the tube is exposed to the rays of a non-luminous source of heat.

If one-half of a tube is surrounded by a water jacket of a higher temperature, and the other half by a water jacket of room temperature, the animals in the warmer part become restless or perish; they are not oriented, however, and consequently crinnot save themselves by moving into that portion of the tube having a lower temperature.

In these experiments the animals were contained in a 
long test-tube $a$ (Fig. 4). The upper half was surrounded by a wide hollow cylinder $b$, the bottom of which was composed of the cork stopper $c$, into which $a$ fitted water-tight. The lower half of $a$ extended into the hollow cylinder $d$. $b$ and $d$ were filled with water to a given height. When the

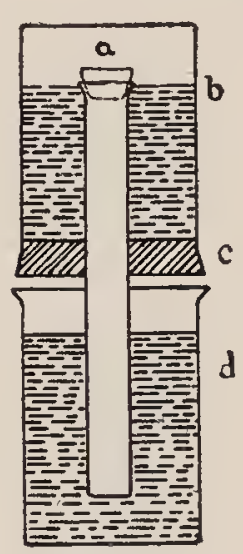

FIG. 4 temperature of the water in the cylinder $b$ was $34^{\circ}$, while that of the water in $d$ was $18^{\circ}$, the animals in the upper part of the tube became very restless, but did not creep into the cooler part of the tube. Nor were the animals oriented when the temperature in the lower part of the cylinder was higher than in the upper. Such an orienting effect of temperature was observed only when an animal came to the boundary between the warmer and the cooler zones at $c$. In such a case the animal moved into the zone having the lower temperature, but not into the other.

By means of diffuse daylight, however, it was an easy matter to drive the animals from a place of lower temperature to one of higher. This is possible because the light orients the animals and dictates to them sharply the direction of their progressive movement, while the same is done by a source of heat only to a slight extent. It was therefore possible to drive the animals from diffuse light into direct sunlight, notwithstanding the difference in temperature.

At low temperatures, even $+10^{\circ}$, it is scarcely possible to demonstrate the heliotropism of fly larvæ. Heliotropic experiments in these animals succeeded best at a temperature of $20-25^{\circ}$.

The orientation of Musca larve toward chemical stimuli. - If on a summer day a piece of meat is set in the open, blow flies collect on it in great numbers and deposit their eggs. There can be no doubt that a chemical stimulus attracts the animals and causes them to lay their eggs. 
Decaying meat has the same attractive effect on the larvæ of flies. If such animals are in a test-tube containing decaying meat, and the tightly fitting cork is loosened a little, the animals which were crawling between the meat and the open end of the tube turn and go back toward the meat. I moistened a small area on a plate by rubbing it with decaying meat. I placed some half-grown larvæ which I had taken from the meat in the middle of this moist surface. They at first crept toward the room side of the plate, but turned when they came to the boundary of the surface smeared with the putrid meat, and remained within it. Not until half an hour later, when the spot had dried completely, did they leave it. When I merely moistened a spot on the plate with pure water, the larvæ did not remain on it.

When I removed the animals from a cadaver and placed them on a glass plate, and brought a piece of decaying meat into their neighborhood, the animals crept toward it, even if in so doing they were obliged to move toward the window; this occurred, however, only when the animals were in the immediate neighborhood of the meat. When they were more than a centimeter and a half away from the meat, they were no longer attracted by it; they then followed the direction of the rays of light and starved in the neighborhood of food. Animals which had not yet tasted food were also attracted by the decaying meat. Fat, even when foul, attracted the animals only slightly or not at all; this is very remarkable, as the female flies are also more readily attracted by meat than by fat. I often placed a piece of horse flesh and a piece of horse fat side by side in the sun. At a time when the flesh was covered with eggs, the fat was almost free from them. It seems, therefore, as though the same chemical stimulus which attracts the larva causes the flies to deposit their eggs. Decaying cheese also attracted the larvæ, but ammonia and assafoetida were without effect. Some volatile 
substance must be formed in the decomposition of the proteids which attracts the Musca larvæ even from a distance.

The contact-irritability of Musca larvo.-It is a wellknown fact that Musca larvæ are inclined to crowd into cracks and crevices in the earth, and it is astonishing through what small cracks the adult larvæ can slip. This irritability might impress a Darwinian as though the animals wished to protect themselves from the light. That this contact-irritability is entirely independent of their heliotropism is shown by the fact that these animals crowd themselves under a completely transparent glass plate, even if by so doing they have to move toward the light.

The animals retain this form of irritability even when put into a vessel of water, in which they soon die. I noticed this phenomenon in feeding tritons with fly larvæ. Small stones lay on the bottom of the vessel, and the larvæ crowded themselves under them as eagerly and as skilfully as if they had always lived under them. The perniciousness of this irritability in the case in question is apparent when we remember that it keeps the animals from reaching the surface of the water again, so that they are drowned.

In these experiments I was struck by the fact that the animals, when placed under the surface of the water, do not swim upward and so avoid death, but swim downward. I cannot explain this fact. Under other conditions positive geotropism cannot be demonstrated in these animals.

The positive heliotropism of flies at the time of sexual maturity.-The fly, which as a larva is negatively heliotropic, is positively heliotropic in the state of sexual maturity. This reversal in the sense of heliotropism in changing to the adult state is not uncommon. Yet it is a striking fact that, while heljotropism is reversed, the orientation toward chemical substances is the same in the female flies at sexual maturity as in the larval state. 
Positive heliotropism can be demonstrated in flies by the same experiments as in plant lice; only it must be noticed that flies are provided with several more kinds of irritability than plant lice, and that in consequence heliotropism may be obscured when other stimuli besides light come into play. In one experiment, for example, I observed

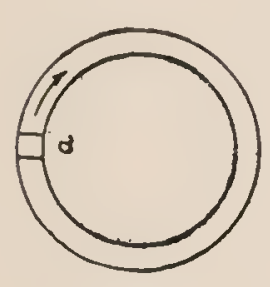

FIG. 5 that light, gravity, and heat were without effect on the flies, because the animals always remained on the cork stopper in the test-tube. Some substance was probably on the stopper that attracted the flies; for when I put the animals into a flask with a clean glass stopper, they reacted to light.

I am indebted to Professor Ernst Mach for a beautiful observation on the influence of light on the orientation of the house fly:

Several years ago I accidentally made an observation which I have never been able to follow further. While adjusting my rotating polarization apparatus in a dark room, by the help of sunlight, whereby a bright quadratic picture $a$ some $16 \mathrm{~cm}$. across (Fig. 5) was rotated three or four times per second in a circle of a radius of $30 \mathrm{~cm}$., a fly $F$ (Fig. 6) happened to enter the bundle of rays $L L$, went through the whole rotation as though stunned, and fell upon the table. I was able to repeat this experiment twice. The fly, which was apparently sound, escaped while I was giving my attention to something else.

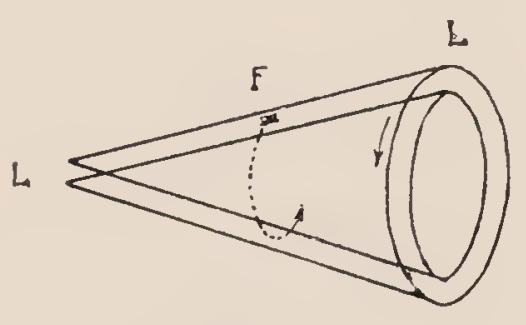

FIG. 6

In this case, then, the same effect was produced by rotating the rays of light as by revolving the fly on a centrifugal machine. ${ }^{1}$

1 Radl has recently come to the conclusion that the reactions of insects on a centrifugal machine are indeed caused by the light. If this is correct, they are identical with Mach's observation. [1903] 
X. THE NEGATIVE HELIOTROPISM OF THE LARV E OF TENEBRIO MOLITOR

The larvæ of a beetle Tenebrio molitor, which can easily be collected in large quantities, are also suitable animals upon which to demonstrate negative heliotropism. When such animals are placed on a plate, they creep to the room side of it; if the intensity of the light is sufficiently great, they remain there. If the plate be covered with dark-blue glass, the result of the experiment is the same. If the plate be covered with red glass, the animals move in the concave edge of the plate both toward the window and away from it; a definite orientation does not occur. Under red glass they behave just as in the dark; under blue glass, just as in the light.

I covered one-half of a plate with blue glass and one-half with red glass, so that the boundary between them lay in the direction of the rays. The animals were distributed equally over the plate at the beginning of the experiment. All the animals in the blue half moved to the room side of the plate, but none in the opposite direction; in the red half they moved in all directions. The animals moved from the blue into the red, but never from the red into the blue. When I covered one-half of the plate with opaque cardboard, the other half with red glass, so that the boundary between them again coincided with the direction of the rays of light, the animals scattered in all directions in the two halves of the plate. After a long time, however, the greater number collected under the cardboard.

The experiments which have been described were made in direct sunlight. If on a dark day the plate is some distance from the window and the light is not very intense, the animals, which at the beginning of the experiment were in the middle of the plate, will gradually creep toward the room side; when, however, they reach the shallow groove in the 
plate, they do not again leave it, and now creep toward the window also. The animals are forced to bring the surfaces of their bodies as much as possible in contact with other solid bodies.

These phenomena are not altered when the plate is covered with blue glass. If, however, it is covered with red glass, the animals, even when in the middle of the plate, move as frequently toward the window as toward the room side. So far as the stereotropism of these animals is concerned, it must be added that the animals collect in the concave edges of dark boxes.

It might be supposed that the function of stereotropism is to protect the bodies of the animals from evaporation as far as possible. I covered one-half of the bottom of a box with a moist cloth and the other with a dry one, and, after putting fifty animals in each half of the box, I placed it in the dark. After two hours not a single animal was found in the moist half of the box. The animals flee from moisture and seek dry spots. Contact-irritability and negative heliotropism determine the habits of these animals, which live protected from the light, in flour.

The negative heliotropism of the larva of June bugs.The behavior of the larvæ of Melolontha vulgaris is quite similar to that of Tenebrio molitor. As they move for the most part while lying on their sides, their orientation takes place rather slowly; nor do they follow in the direction of the rays of light as sharply as do the animals which have been described above. They flee from the light and move from the window to the room side of a vessel.

The following experiment, which also serves to give an idea of the time required for experiments on these animals, shows that only the more refrangible rays are of chief importance in bringing about the heliotropic phenomena: At 10:40 o'clock I placed twenty-three larvæ in the middle 
of a round plate covered with blue glass. The animals moved to the room side of the plate, tried to creep over the edge, and at 10:45 came to rest on the room side. I waited five minutes, and at 10:50 substituted red glass for the blue. The animals scattered equally over the whole plate, and at 11 nine animals were on the window side, the rest about uniformly scattered over the whole plate. I then substituted blue glass for the red. At 11:07 all the animals were collected on the room side of the plate. At 11:10 I again covered them with red glass. The animals immediately began to creep over the plate in all directions. At 11:20 twelve animals were collected near the window, six in the middle, two on the side, and three on the room side of the plate. I kept the plate covered with red glass, and watched to see whether after a time the rays going through the red glass would not also bring about an orientation. No change occurred in the course of the next hour. Gradually, however, more and more animals moved to the room side of the plate, and at 3:30 all but five animals were collected here. The animals, therefore, finally show a negative heliotropism under red glass also. The rays passing through red glass are therefore similar in their effects to those which go through blue glass, only they are not so effective. In this respect the behavior of these animals corresponds with that of plants.

The larvæ burrow into the ground. Negative heliotropism may co-operate here, but stereotropism is without doubt the chief factor concerned.

The question arises whether it is not geotropism which causes the animals to bore into the ground, as in the case of roots. In order to determine this I made the following experiment: I filled a hollow cardboard cylinder, some $5 \mathrm{~cm}$. in diameter, with earth. The cylinder was about $20 \mathrm{~cm}$. high. I fastened the cylinder on a stand, with its longitudinal axis vertical, and brought it so near to a table that it 
just touched two larve lying upon it. I also placed two larvie on top of the cylinder. If the animals were negatively geotropic, the upper animals should have buried themselves more quickly than the lower. But the opposite was the case. After forty-five minutes the lower animals had burrowed upward so that they were completely out of sight; the upper were not buried until an hour later. Therefore, even though they may be negatively geotropic, for which I have as yet no proof, the contact-irritability of these animals determines that they shall burrow into the ground.

\section{THE DISTRIBUtion OF HELIOTROPIC PHENOMENA IN THE} ANIMAL KINGDON

The experiments which have thus far been described were carried out on insects.

So far as experiments on representatives of the other divisions of the animal kingdom are concerned, I have confirmed the identity of animal with plant heliotropism on crabs (Gammarus locusta, Cuma Rathkii), naked snails and worms (leeches, planarians, earth-worms and others). Experiments on infusoria are already sufficiently complete to show that Sachs's laws of heliotropism also hold good for them. ${ }^{1}$

Investigations have not yet been made on Cœlenterates and Echinoderms; Trembley's experiments on Hydra, however, show that in their case also the relation is the same; at least it seems to me that Trembley's experiments cannot be interpreted unless we assume that the progressive movements of Hydra are determined by the direction of the rays of light.

I used the following method with aquatic animals: To prove that the direction of the rays determines the direction of the progressive movement, I used a long, four-cornered glass box, one wall of which was made of a watch-glass. The 
convex side of the watch-glass was turned outward. When direct sunlight fell upon the glass, the rays were focused a few centimeters behind the glass wall. Notwithstanding this fact, the positively heliotropic animals moved in the direction of the rays from the room side of the glass box through the focal point to the front of the box, although the intensity of the light was the greatest in the focus. This could be shown very beautifully in some tiny, positively heliotropic worms I found in the brackish water at Kiel, but whose identity unfortunately I failed to determine.

Positive heliotropism is encountered more often in the plant kingdom than negative heliotropism. It is worth while to mention the fact that positive heliotropism appears to exist in more species in the animal kingdom also than does negative heliotropism.

All caterpillars and Lepidoptera, whether they fly by day or night, can, according to my observations, be considered positively heliotropic. Thus far I have tried in vain to find negatively heliotropic Lepidoptera or caterpillars. The great majority of the other winged insects are also positively heliotropic.

We also encounter positive heliotropism in animals which live in water, and even in mud, and which therefore can never profit by light. I was much interested in some observations I made in this direction on a small Crustacean (Cuma Rathkii) which lives on the bottom of the bay of Kiel. The animal can be fished out of the mud in which it buries itself only with a dragnet. Notwithstanding this fact, the animal is strongly positively heliotropic. When I kept these small crabs in a glass vessel and allowed light to fall upon them from one side only, the moving animals collected at the side of the vessel nearest the light. The resting animals were oriented, and turned their oral poles toward the source of light and their median planes in the direction of the rays. 
When the source of light or the vessel was carefully turned about, the animals changed their orientation until their median planes were again in the direction of the rays of light. The directing force of the light exhibited itself here in the same manner as in the Euglenæ in the experiments of Stahl.

I next placed a small glass box filled with mud in the same vessel with the crabs. The animals did not scent the mud; at least not one of them moved into the box containing the mud. When I disturbed the animals (by touching them with a pencil), they first swam upward and then, if I did not disturb them further, slowly fell to the bottom. If an animal happened to fall upon the mud, it immediately became lively as soon as it touched the mud. It burrowed into it eagerly, after which it was impossible to get the animals to react to light. The other animals which fell to the glass bottom of the vessel remained inactive.

Thus we see that contact with the mud had a greater effect than light; contact-irritability is more intense than heliotropism. It is in this way that it happens that the animal, besides being a poor swimmer, lives away from the light in spite of its positive heliotropism.

\section{XiI. THE DEPENDENCE OF THE ORIENTATION UPON THE FORM OF THE BODY}

In the introduction I have called attention to the fact that the orientation of an animal toward light, like every phenomenon of irritability, is determined by two factors: first, external causes - in this case the light-and, second, internal causes, namely, the structure of the animal.

So far as the structure of the animals is concerned, we are dealing in this paper exclusively with animals whose bodies consist of two morphologically symmetrical halves, and which have morphologically different ventral and dorsal 
surfaces and morphologically different oral and aboral poles. We disregard all other detailed morphological peculiarities, because those mentioned suffice to explain the orienting movements of an animal, as they do for the movements of plants. The distribution of irritability on the surface of an animal corresponds to the above-mentioned morphological relations. Elements at the surface of the body symmetrically situated with reference to the median plane have equal irritabilities. This condition compels the animal to orient itself toward a source of light in such a way that the rays of light strike the symmetrical points in the body at equal angles; this is the case when the animal places its median plane in the direction of the rays of light. Points on the dorsal or ventral surface equidistant from the median plane have unequal irritabilities, the irritability being in general the greater the nearer the points are to the oral pole. In the same way, the irritability of a dorsal element is different from the irritability of the opposite ventral element. If these assumptions regarding the connection between irritability and the main structure of an animal are correct, it follows, without further discussion, that an animal with bilateral symmetry is compelled to place its median plane in the direction of the rays of light and to move in this direction either toward or away from the source of light. We must therefore prove that the described distribution of irritability on the surface of an animal is not fiction, but reality.

1. The oral pole of an animal is more irritable heliotropically than the aboral pole, no matter whether the animal has eyes or not.

I have already mentioned that the blind adult Musca larva immediately places the entire median plane of its body in the direction of the rays of light when sunlight strikes only its oral pole. When, however, the oral pole remains in the 
shade, while the aboral pole is exposed to the sun, the animal does not bring its median plane into the direction of the rays of light and does not move in this direction, although it may become very restless. Light therefore prescribes the direction of the progressive movements in these animals when it strikes the oral pole, but it does not have this effect when the light falls only upon the aboral pole.

I have already called attention to Engelmann's observation that Euglena viridis reacts clearly only when the light falls upon the oral pole. Euglena viridis possesses a pigment spot at this pole which is called the "eye-spot." Physiologically this expression is a very unhappy one, as we do not know that this spot has anything in common with the functions of our eye. Engelmann, however, expressly states that the most sensitive spot of the Euglena is not the pigment spot, but the colorless protoplasm lying just in front of it.

The earthworm has no eyes. Hoffmeister found that the animals recede from the light when the anterior end of the body is illuminated. If, however, the oral pole is shaded and the rest of the body is illuminated, no effect is produced. Darwin repeated and corroborated this experiment. ${ }^{1}$

Fresh-water Planarians have eyes and are negatively heliotropic, but their sensitiveness to light is not very great. I cut Planarians in two in the middle. The oral piece reacted to light soon after the operation, just as the whole animal. Not once, however, did I see indications of a heliotropic movement in the aboral piece before regeneration had set in $^{2}$ (which is very complete in these animals), even though the aboral piece was by no means inactive, but crept around very energetically in the glass and reacted promptly when touched. When I placed both pieces on the window

1 DARwIx, Die Bildung der Ackererde durch die Thätigkeit der Würmer, trans. Dy CARUs, 1882, pp. 11 ff.

2 I observed different facts in an American fresh-water Planarian. [1903] 
side of the vessel, the oral piece moved toward the room side, but not the aboral piece. When the oral piece moved from the room side toward the window, it soon turned about. Under similar conditions the aboral piece continued to creep until it reached the window. When the vessel containing the animals was carefully reversed, the aboral animal was not affected, but the oral animal immediately moved toward the room side.

It can easily be shown that in leeches the head, which contains the eyes, reacts more energetically toward light than the aboral pole. If some small stones are lying on the bottom of a beaker which contains such animals, and the vessel is suddenly illuminated, the animals push their heads under the stones, while the aboral pole remains at rest even though exposed to the light. It is astonishing to notice how long after the illumination the reaction appears. It is not unusual for thirty to seventy seconds to elapse between the illumination and the beginning of the movement. Hoffmeister observed a still longer latent period in the case of the earthworm. It would be unnecessary to show that in animals which possess eyes the oral pole is more sensitive toward light than the aboral. We may therefore-accept it as certain that the oral pole of an animal is more sensitive toward light than the aboral, whether the animal does or does not possess eyes.

In consequence of this fact, it is difficult for an animal to move perpendicularly or obliquely to rays of light emanating from a sufficiently intense source, for, as the oral pole is more sensitive than the aboral, the former must turn more energetically toward or away from the source of light (depending upon whether the animal is positively or negatively heliotropic) than the aboral.

2. The heliotropic irritability is also different on the ventral and dorsal surfaces of a dorsiventral animal. To 
my knowledge, no observations on this subject have as yet been made on animals.

Planarians and leeches afford an example of the difference between dorsal and ventral irritability. In leeches the ventral surface, which has no eyes, is more sensitive to light than the dorsal surface. It has already been said that this animal leaves the dorsal side of its aboral pole exposed to the light, if only its head is protected from the light. Such animals stick to the side of a beaker, so that their ventral surfaces, which carry the suckers, are directed outward. If diffuse light of a sufficient intensity falls upon the ventral surfaces of the animals, most of them leave the window and move to the room side. The animals then turn their dorsal surfaces to the light.

In this case, as in all the others, only the more refrangible rays are chiefly active. When the animals are covered with red glass, orientation does not follow, or only after some time. If blue glass is held over them, the orientation takes place just as in diffuse daylight.

The difference between the irritability of the ventral and the dorsal surfaces of dorsiventral animals is therefore comparable with that between the oral and the aboral poles.

3. The dependence of the irritability of a dorsiventral animal on the symmetry of its body must yet be discussed. Those elements of the body of a dorsiventral animal which occupy symmetrical positions with reference to the median plane have equal irritabilities.

The facts which prove this are to such an extent objects of daily experience that a brief allusion to them will suffice. If a touch on one side of the animal calls forth a movement to the left, then the same stimulus applied to the opposite symmetrical spot on the body will cause the same amount of movement to the right. An object appearing in the right field of vision causes the same amount of movement as one 
appearing in the left at the same distance from the median plane. In short, we can say that the symmetrical plane of an animal from a morphological standpoint is also the symmetrical plane from a physiological standpoint.

This distribution of irritability on the surface of an animal determines the orientation of dorsiventral animals toward a source of light. If the median plane lies in the direction of the rays of light, the symmetrical points of the surface of the animal are struck by the rays at an equal angle. The effects of the stimuli on the right and left halves of the body annihilate each other, since they are equal in intensity and opposite in direction. The light can therefore produce no tendency to turn to the right or the left. When, however, the median plane is oriented obliquely toward the source of light, unequal forces act upon symmetrical elements, and a tendency to turn must arise which continues until the median plane coincides with the direction of the rays of light.

This dependence of irritability on the form of the body causes Musca larvæ to move away from the source of light precisely in the direction of the rays, and plant lice to move just as precisely in the direction of the rays toward the source of light. The heliotropic movements of an animal are therefore dependent on the symmetrical relations of its body, in the same manner as was shown by Sachs to be the case in plants.

To show how far these conceptions of heliotropic phenomena in animals differ from the prevailing notions on the subject, especially those of the Darwinians, I shall give the views of Romanes on this subject. Romanes mentions the well-known facts that insects of all kinds fly into the flame, that many birds are attracted by the light of lighthouses, and fishes by the lanterns. He explains the phenomenon as follows: "The habit must be attributed to mere curiosity, or 
desire to cxamine the new and striking object;" and then quotes some remarks which he found in the manuscripts of Darwin: "Query: Why do moths and certain gnats fly into candles, but not into the moon when the same is at the horizon? I noticed long ago that they fly much less frequently into candles on a moonlight night. When a cloud passes over the moon, they are again attracted by the candle." Romanes believes that: "The answer must be that the moon is a familiar object, which insects consider as a matter of course, and so have no desire to examine it."

As we have seen, it is not the "new and striking" object and "the desire to examine it" which drive the insects to the lamp, for they are attracted, as I have shown, also by the natural source of light, the sun. No reason seems to exist to my mind for believing that the moon is a more familiar object to the insects than the sun. I cannot well see, however, how Romanes harmonizes the phenomena of negative heliotropism in animals with "the desire to examine unfamiliar objects." The history of science has taught us that confusion always reigns when anthropomorphic motives are brought into scientific research. Before the time of Galileo a body sinking in a fluid "sought its place."1 Galileo and his followers put an end to the sovereignty of this psychology, at least in inanimate nature. Mankind has had no reason to regret this revolution. In biology, however, even at this date, protoplasmic substances still move toward the source of light "because of curiosity."

\section{SUMMARY OF RESULTS}

I shall conclude by summarizing the more important results of my investigation:

I. The dependence of animal movements on light is in every point the same as the dependence of plant movements on the same source of stimulation.

1. See MACH, Gsschichte der Mechanik, 1st ed., p. 117. 
1. The direction of the median plane, or the direction of the progressive movements of an animal, coincides with the direction of the rays of light. ${ }^{1}$

2. The more refrangible rays of the visible spectrum are exclusively or more effective, than the less refrangible rays, in causing the orientation of the animals, as is also the case in plants.

3. Light of a constant intensity acts as a constant stimulus in animals as well as in plants.

4. The intensity of the light is of importance in animal heliotropism, in so far as only light of a certain intensity can cause heliotropic movements, and in so far as with an increase in the intensity the orientation of the animals toward the source of light becomes more exact. Direct sunlight causes winged insects (ants, Lepidoptera, plant lice, etc.) to fly, while diffuse light usually causes them only to creep. Positively heliotropic animals will move toward the source of light, even if in so doing they go from places of greater intensity of light to places of less intensity; negatively heliotropic animals move away from the source of light, even if in so doing they pass from regions of less intense light to regions of greater intensity.

5. Heliotropic movements occur only between certain limits of temperature. An optimum temperature lies beween these two limits at which the heliotropic movements occur most rapidly and precisely. This holds true also in plants.

II. The orientation of an animal toward a source of light depends on the form of the body, just as the orientation of a plant to light depends on the form of the plant.

1. Symmetrical points on the surface of the body of dorsiventral animals possess equal irritabilities. ${ }^{2}$

1 If there is only a single source of light. If there are two sources of light of different intensities, the animal is oriented by the stronger of the two lights. If their intensities be equal, the animal is oriented in such a way as to have symmetrical points of its body struck by the rays at the same angle. [1903]

2 Equal in magnitude, not in direction. [1903] 
2. The heliotropic irritability of the oral pole of an animal is different from the irritability of the aboral pole, and is generally greater than the heliotropic irritability of the aboral pole.

3. The irritability of the ventral surface is different from the irritability of the dorsal surface.

These three conditions taken together cause dorsiventral animals to place their median planes in the direction of the rays, and to move toward or away from the source of light in this direction.

4. Eyeless animals (such as the larvæ of Musca vomitoria) behave in this respect like animals having eyes.

III. The heliotropic irritability of an animal manifests itself frequently only at certain epochs of its existence.

1. In winged ants this epoch is the time of the nuptial flight.

2. In plant lice it is the time when wings are present.

3. In the larvæ of Musca vomitoria negative heliotropism is most prominent when they are fully grown.

4. In a large number of animals the sense of heliotropism is of the opposite kind in the larval and the adult states.

5. Both the night and day Lepideptera are positively heliotropic, and their heliotropism is similar to that of every other positively heliotropic animal. The period of sleep of the night Lepidoptera, however, falls in the daytime, and only for this reason is their heliotropism manifested exclusively at night.

IV. In many animals heliotropic irritability is connected with sexuality. Aside from the nuptial flight of ants, the fact must be mentioned here that in ants and Lepidoptera the males are heliotropically more sensitive than the females.

V. The behavior of an animal depends on the sum total of its different forms of irritability. In this way it may happen that Cuma Rathkii and the caterpillars of the willow. 
borer, which live in the dark, are positively heliotropic without deriving any benefit from this form of irritability.

VI. There is one form of irritability widely distributed throughout the animal kingdom, which has been studied but little, and which can easily be confounded with negative heliotropism. It consists in many animals being compelled to orient their bodies against the surfaces of other solid bodies in a certain way, or bringing their bodies in contact with other solid bodies on as many sides as possible (stereotropism). Certain animals seek only the concave corners and edges of boxes (Forficula auricularia, ants, Amphipyra, the larvæ of Musca vomitoria, etc.); while others fasten themselves only to the convex edges and corners (caterpillars of Porthesia chrysorrhœa).

VII. A non-luminous source of heat may influence the orientation, but generally it is not able to prescribe the direction of the progressive movements of animals. In this way it happens that animals which move away from a source of heat may be forced by the light to move from diffuse light into sunlight, and to remain exposed to the high temperature of the sunlight, even though this may cause their death. The influence of a non-luminous source of heat can best be compared to the influence of a weak source of light, which is just sufficient to hinder a negatively heliotropic animal from going toward the source of light, but is not sufficient to force the animal to move accurately in the direction of the rays.

We have yet to draw a conclusion from the results of these experiments, which could not be formulated until now. We have seen that the heliotropic movements of animals possessing a nervous system are determined in all respects by the same external conditions and depend in the same way on the external form of the body as do the heliotropic movements of plants, which have no nervous system. These heliotropic phenomena cannot therefore depend upon specific characteristics of the central nervous system. 
XIV. ADDENDUM: SOME FURTHER EXPERIMENTS ON THE GEOTROPISM OF INSECTS

As I have several times had occasion in this volume to mention the influence of gravity on the orientation of animals - a subject which in this form has not yet been discussed in physiological literature - it may perhaps be desirable to add a few further facts on animal geotropism. I must say beforehand, however, that my experiments in this field are not yet completed, and that I intend to return to this subject.

1. I have found that caterpillars (for example, Bombyx neustria) when placed in a hollow vessel creep vertically upward. When we wish to pour such caterpillars out of a vessel, we employ a method opposite to that used in pouring out a liquid; we must hold the mouth of the vessel upward. When such caterpillars are contained in a glass vessel, the diffuse daylight entering from above in itself would bring about this effect; I therefore made this experiment in wooden vessels. When the opening of the vessel was directed downward the animals crept upward, and not an animal escaped from the vessel. Geotropism, however, like heliotropism, is especially evident only at certain epochs in the life of the animal; for the geotropic experiments were not at all times successful even in the same animals.

2. Small beetles, particularly Coccinella, which can always be procured with ease in great numbers, were placed in a wooden box, and to protect them from the effects of light I put the box in a dark closet. The animals, which were at first scattered over the whole box, were found the next day collected at the highest point in the box, on the upper side, where they remained. When I turned the box about, they changed their orientation and moved again to the top. The behavior of Coccinellidæ and other beetles (particularly leaf 
beetles) is somewhat remarkable, as many other animals, such as caterpillars, plant lice, etc., do not react geotropically after they have been kept in the dark for some time.

3. Another phenomenon can be observed in Coccinellidæ, to which I have already referred in my former paper on the "Orientation of Animals toward the Center of Gravity of the Earth." Cockroaches, for example, usually assume a position on the vertical walls of boxes; they never remain for any length of time on the horizontal bottom, where their ventral surfaces are turned toward the center of gravity. If, for example, the roaches are scattered about on the four vertical walls of a box, and the box is carefully and slowly turned through an angle of $90^{\circ}$ on a horizontal axis, only those animals whose ventral surfaces are turned toward the center of gravity in the new position begin to move. They leave the plane on which they were up to the time the box was turned and again seek a vertical plane from which to suspend themselves. Yet the animals on the other three vertical walls remain at rest while the box is turned. This seems to indicate that the animals cannot turn their ventral surfaces toward the center of gravity for a long time. Whether this means that the animals' extremities can bear the pull of the load of the body better than its pressure, I cannot say. The same phenomenon is also observed in Coccinellidæ. If the box is placed obliquely, the two oblique planes on which the animals turn their ventral surfaces toward the center of gravity are first abandoned.

4. Aside from the peculiarity which has been mentioned, the Coccinellidæ, like roaches, are found hanging on walls in all possible orientations toward the center of gravity of the earth. Great differences are therefore found in this regard also, for there are animals (such as the newly hatched Lepidoptera) which put the median plane of their bodies in 
the direction of the vertical and turn their heads upward. There are, however, also animals which orient themselves in exactly the reverse manner and turn their heads downward. To this class belongs the garden spider, which we find hanging in this position in the center of its web for hours. I found the same behavior in one of the Diptera, which I have not yet classified.

5. If a beetle or a house fly (from which the wings have been removed) is placed on the disk of a centrifugal machine, which is rotated slowly, the insect turns its body around the same axis, but in an opposite direction to that of the revolving plate.

If the velocity of the machine is increased, these compensatory movements cease. These animals therefore behave in this respect exactly as Mach claims vertebrates behave which possess a labyrinth. ${ }^{1}$ But while the movements of vertebrates continue for some time after the movement of the centrifugal machine has ceased, but in a sense opposite to those occurring during rotation, I have never been able to bring about these compensatory after-movements in insects. ${ }^{2}$

6. When one hemisphere of the brain of a house fly is removed the same disturbances in orientation appear as after the same operation on a rabbit. The fly from which the left hemisphere has been removed moves continually toward the right in its progressive movements. These deviations are greater or less according to the success of the operation. I showed in an earlier paper that the turn-table reactions of dogs and rabbits deprived of a hemisphere might be unsymmetrical. If a fly which has lost the left half of its brain is placed on a rotating disk, we find that on turning the disk in the direction of the hands of a watch as seen from above,

$1 \mathrm{MACH}$, Grundlinien der Lehre von den Bewegungsempfindungen (Leipzig, 1875).

2 The observations of Lyon and of Rádl suggest the possibility that these phenomena depend upon the eyes of these animals. When the eyes are removed or blackened the reactions cease. [1903] 
the fly makes slight or only weak compensatory movements Yet when the disk is turned in the opposite direction the fly reacts very promptly (apparently even better than before the operation). After destroying both hemispheres or amputating the head I no longer obtained compensatory movements in the fly. The experiments of Mach show that the compensatory movements of vertebrates emanate from the head; according to Mach, the labyrinth is to be considered the essential organ. Such an organ, however, does not exist in the head of a fly.

7. If the halteres are removed from a fly, it can no longer fly upward; in the attempt to fly it immediately falls to the ground, where it frequently tumbles about. GleichenRusswurm established this fact during the last century. I found that such a fly reacts normally on the centrifugal machine. The destruction of the halteres does not therefore have the same effect as the destruction of the labyrinth in frogs, birds, or mammals, in which, according to the experiments of Högies and Schrader, compensatory movements cease when the labyrinth is destroyed. The conjecture expressed by others, and by me in my first publication, that the direction of sound has an influence on orientation has thus far led me to no new facts.

8. I have not yet been able to demonstrate compensatory movements on the centrifugal machine in caterpillars, Musca larvæ, and snails. 


\section{II}

FURTHER INVESTIGATIONS ON THE HELIOTROPISM OF ANIMALS AND ITS IDENTITY WITH THE HELIOTROPISM OF PLANTS ${ }^{1}$

IN a former paper I showed that the dependence of animal movement upon light is identical with that of plants on the same source of stimulation. ${ }^{2}$ I showed that the law put forward by Sachs for the heliotropism of plants, namely, that the direction of the rays of light determines the orientation, holds good also for animals. Freemoving animals are compelled to execute their progressive movements in the direction of the rays of light, as is the case with the swarm-spores of certain Algæ. It was further proved that the more refrangible rays of the visible spectrum are the rays that are solely, or at least chiefly, effective in bringing about the movements of these animals; as is the case in the heliotropic movement of plants. After I had proved the identity of this relationship point for point, I believed it permissible to designate these reactions of animals by the same term as that used for the identical reactions of plants, namely, "heliotropism."

At that time, however, I had proved this identity only in the case of free-moving animals. The task still remained to ascertain and investigate the influence of light upon the orientation of sessile animals, and to decide whether the influence of the light is in this case also similar to that upon sessile plants. It is known that in plants the direction of the

1 Pflügers Archiv, Vol. XLVII (1890), p. 391.

2 Part I, p. 1. See also LOEB, Sitzungsberichte der Würzburger physikalischmedicinischen Gesellschaft (1888); Groom UND LoEB, Biologisches Centralblatt, Vol. X (1890). 
light rays determines the orientation of the organs of the plant. It is characteristic of the organs of sessile plants that heliotropic curvatures are produced when the plant is illuminated from but one side. A growing stem continues to bend when illuminated from one side only until the growing tip lies in the direction of the rays of light. Progressive movement in the direction of the rays of light, which is the rule for free-moving animals and plants, is of course impossible for sessile organisms. Everyone who has cultivated flowers in a room has no doubt observed the heliotropic bendings in the plants. The question now arises whether these heliotropic curvatures can also be produced in sessile animals when illuminated from one side only. I shall show in the following pages that this is, indeed, the case.

1. The experiments described here were made on the large marine Annelid, Spirographis Spallanzanii. It lives in a tube which is quite flexible, yet sufficiently rigid to keep the animal in a definite position. The tube is formed from the secretions of the animal. The aboral end of the tube is fastened (by a secretion) to stones or other solid objects. The gills of the animal, which are arranged at its anterior end in several spiral turns radial to the longitudinal axis of the animal, are usually found unfolded and projecting beyond the open end of the tube. As the tube is almost impervious to light, the latter will act chiefly upon the gills. So far as we know at present, the animal has no eyes.

The animal can move freely inside the tube, the inner surface of which is perfectly smooth, and can be removed from it without the slightest injury by cutting open the tube. I have occasionally seen the worm leave the tube of its own accord, when the water in the aquarium became bad.

The layman seeing these animals in the tubes with their 
gills fully unfolded takes them at first for plants bearing a palm-like crown (the gills) upon a long naked stem (the tube). A slight jar, however, causes the animals to draw back their gills rapidly into the tubes.

When the animal is taken from the sea and kept in an aquarium, it is at first indifferent to the light. This continues until the animal has attached itself by its foot to the bottom of the aquarium - a period often of several days. As soon as this has taken place, however, the orienting influence of the light begins to be noticeable. If light falls upon the animal from one side only, heliotropic curvatures make their appearance in the tube. The animal turns its oral pole toward the source of light and bends its tube until the axis of its radially expanded gills lies in the direction of the rays of light. The animal maintains this orientation as long as the direction of the rays of light remains unaltered.

2. To test more accurately to what extent the direction of the rays of light determines the orientation of the animals, I put them into an aquarium which stood at the window, and which could be completely screened from the light by a zinc box. The outlines of the aquarium are indicated in the drawings (Figs. 7 and 8) by black lines, the outlines of the zinc box by dotted lines. The wall abcd of the zinc box could be moved vertically upward, so that the amount of light entering the aquarium could be regulated. The zinc box, the walls of which were painted black on the inside, was so placed over the aquarium that the movable wall was on the window side of the aquarium. If this wall was raised only slightly; as shown in Fig. 8, the rays entered the aquarium almost horizontally. When it, was drawn farther up, as in Fig. 7, rays entered from above in addition to the horizontal rays. These were more intense than the rays entering horizontally.

On December 14, 1889, I put nine vigorous specimens of 
Spirographis Spallanzanii, each about $15 \mathrm{~cm}$. long, on the bottom of the aquarium, with the longitudinal axes of their tubes perpendicular to the plane of the window. Eight of them lay with their oral poles toward the room side efgh (Fig. 7) of the aquarium; one with its oral pole toward the window side. The first two days passed without any change

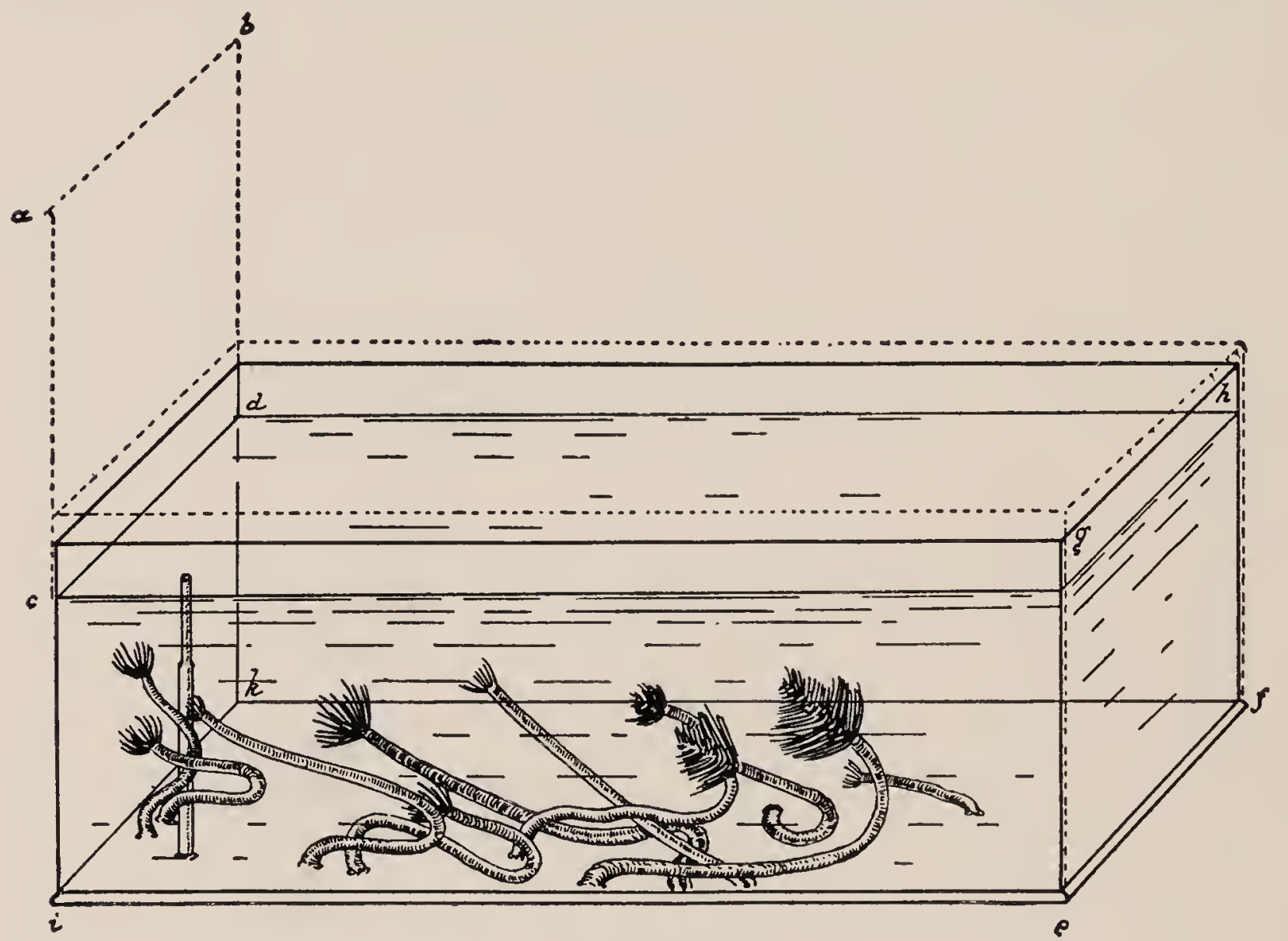

FIG. 7

in the orientation; the animals first attached the aboral ends of their tubes to the floor of the aquarium. In the course of the third day the tubes of six of the animals, which were placed with their oral pole toward the room side, began to bend in an almost horizontal plane, the concavity of the curvature being directed toward the window. The other two animals, which had likewise been placed with their heads toward the room side, first elevated the head end and then curved the tube concavely toward the window. Finally, the ninth animal, which I had placed in the aquarium with its head toward the window, raised its head a little. 
Within the next few days the six first-mentioned animals further elevated their heads, so that the animals on December 22-eight days after being placed in the aquariumwere all similarly oriented toward the light. The head was directed toward the window, and the axis of symmetry of the gills which were exposed to the light lay in the direction of the more intense rays of daylight which entered from

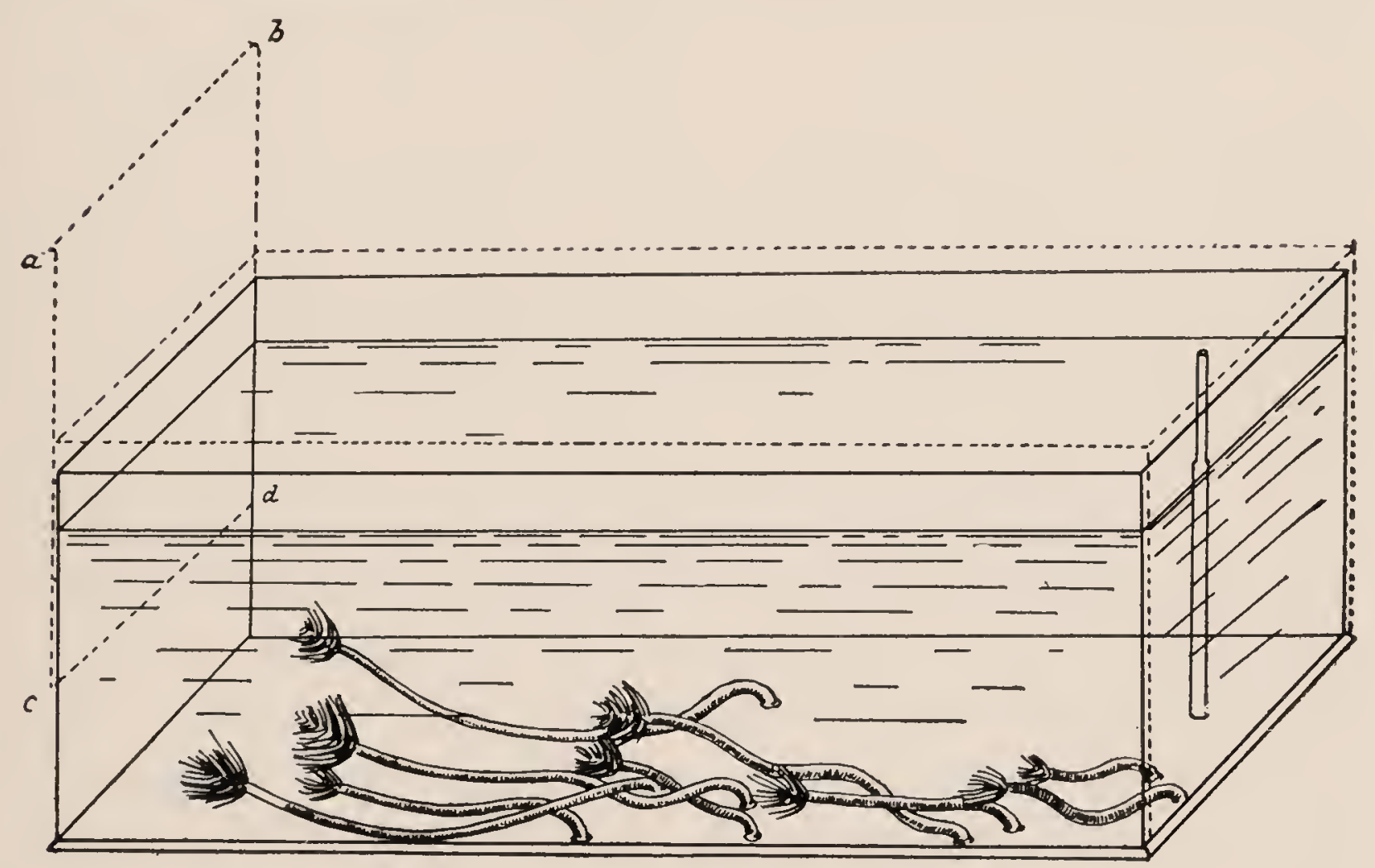

FIG. 8

without and above. I waited to discover whether this orientation would last. The aquarium remained undisturbed until February 16, 1890 ; that is, for more than two months. The animals also did not change their positions, as indicated in Fig. 7.

3. On the afternoon of February 17, 1890, the aquarium was turned $180^{\circ}$ about its vertical axis, and the zinc box was again inverted over the aquarium so that the movable end was directed toward the window. By turning the aquarium around in this way, the heads of the animals, which had been until then directed toward the source of light, were suddenly turned toward the room side of the 
aquarium. My object in turning the aquarium around was to see whether a change in the direction of the rays of light would cause the animals to reverse their heliotropic curvatures and to turn their heads again toward the source of light. There was no change during the course of the afternoon and

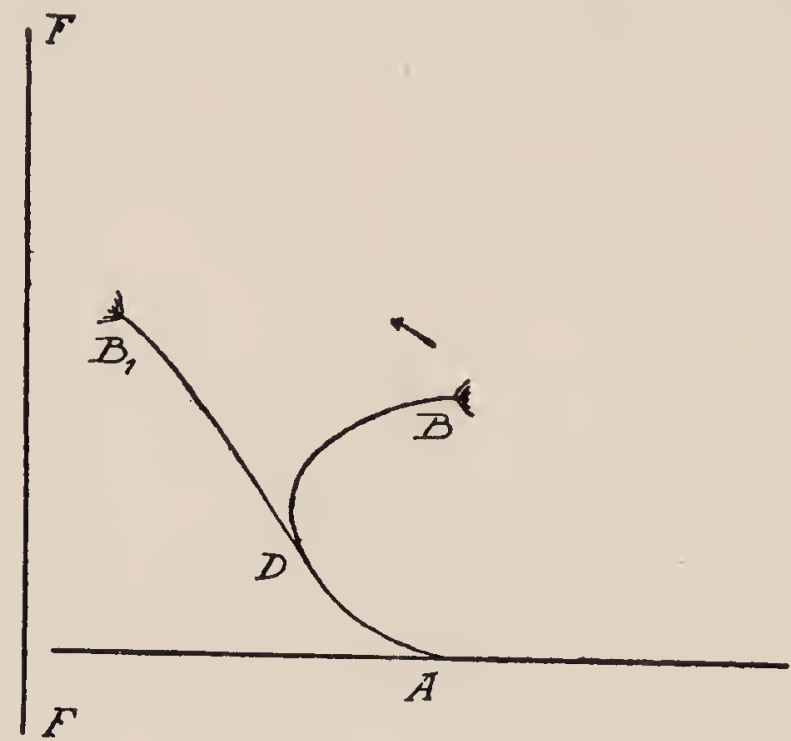

FIG. 9 night. But toward noon of the following day I found two animals, which in the morning had still been in the position $A B$ (Fig. 9), in the position $A B_{1} ; F$ indicates the plane of the window. The portion $D B$ of the tube had described the surface $D B B_{1}$ about the point $D$ as center. A similar change in the orientation of all the remaining animals took place during that and the following day. In this experiment the direction of the rays of light was modified somewhat; the wall abcd was left quite low, so that almost nothing but horizontal rays entered the aquarium (Fig. 8). I wished to determine whether the animals would continue to follow the direction of the rays and so assume an almost horizontal position. This did, indeed, occur. On February 22, 1890, five days after reversing the aquarium, the orientation was accomplished, as indicated in Fig. 8. The animals had turned their heads toward the source of light, and the axes of their gills lay almost horizontally in the direction of the rays of light. I left the conditions of the experiment unchanged until toward the end of March, and during all that time the animals maintained their orientation.

4. If the rays of light fall vertically from above into the aquarium, Spirographis directs its tube vertically upward, exactly as a stem grows vertically upward into the open air. 
This experiment was made in another aquarium, in which the light rays entered chiefly from above. The animals in the large aquarium of the zoölogical station at Naples are usually found mainly in this position; the light enters this aquarium chiefly from above. Here, however, where freeswimming forms easily disturb the orientation of Spirographis, it is not always so perfect as when all possible disturbing causes are avoided, as in an aquarium used only for such experiments.

5. It follows from these experiments that gravitation exerts only a slight effect, if any, upon animals which are subjected simultaneously to the effects of light and gravity. It was, however, necessary to discover whether a geotropic erection of the animals would not occur under the influence of gravity alone in a completely darkened room.

On March 21, 1890, I placed a large number of Spirographis in a horizontal position upon the floor of an aquarium in the dark room. On March 24 most of the animals had attached themselves by their aboral ends to the bottom of the aquarium. The oral ends of the tubes were then elevated until the gills no longer touched the bottom of the aquarium. The axis of the spiral did not stand vertically (as was the case when light fell vertically into the aquarium, or as should have been the case had the animals been geotropically irritable), but only at a slight angle from the horizontal. The animals remained in this position until the end of the experiment, which was interrupted in the middle of April. Gravity therefore has no important influence upon the orientation of Spirographis Spallanzanii.

6. The contact-irritability of the gills is manifested by the fact that they bend away from solid surfaces. This form of irritability can modify the result of the heliotropic experiments upon the animals. I placed several of the animals upon the floor of an aquarium which was so shallow 
that the animals could not erect themselves. They were so placed in the aquarium that their longitudinal axes lay perpendicular to the side $a b$ (Fig. 10) of the aquarium, and their pedal extremities $M$ touched the glass wall $a b$. The side $a$. faced and was parallel to the plane of the window. The

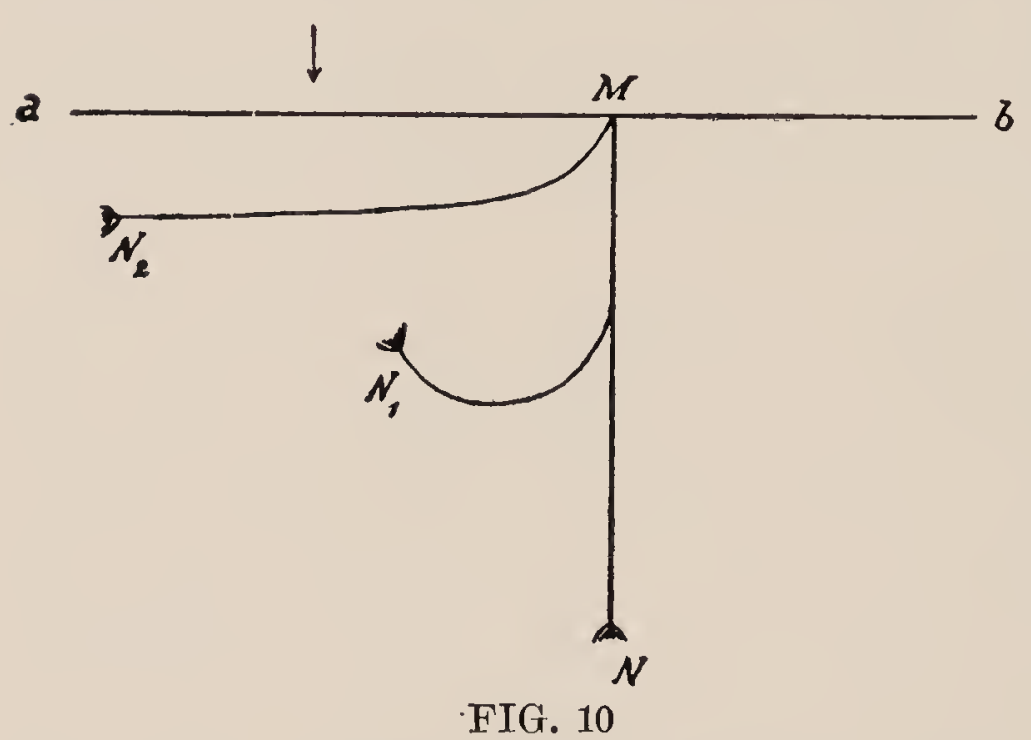

animals fastened themselves to the wall $a b$, and then began to react, in their characteristic way, to the light, by which the head was turned and the tube became concave toward the source of light. The tube $M N$ assumed the position $M N_{1}$. As soon, however, as the tentacles touched the glass wall $a b$, the tip $N$ turned away from the glass wall. The heliotropic bending gradually affected all the elements of the tube $M N$, so that the Spirographis finally reached the position $M N_{2}$, in which it remained throughout the period of observation-four months.

I repeated this experiment a number of times, always with the same result.

7. The heliotropic phenomena of Spirographis took place both in direct sunlight and in diffuse daylight. The minimum light intensity just sufficient to bring about these phenomena is very small. I have not yet studied the effect of rays of different refrangibility in producing these phenomena. Since thus far the more refrangible rays have proved to be the most effective heliotropically both in plants and animals, it is to be suspected that Spirographis also will prove no exception.

8. As is well known, Sachs has formulated the law that 
radial plants are orthotropic; $i$. e., they place their longi-tudinal axes in the direction of the rays of light, or of gravity. It will have occurred to the reader that Spirographis, the body of which, like that of all Annelids, is built on the dorsiventral and not on the radial plan, reacts toward the light as a radial plant organ. I have, however, already emphasized the fact that only the radially arranged gills of the animal are exposed to the light, while the remainder of the animal is inclosed in the tube. These observations, therefore, show that a radial animal organ also obeys the law of orientation established by Sachs for plants (even though Spirographis possesses a central nervous system, which the plant does not).

It is also of physiological interest that the respiratory organ of Spirographis is so highly sensitive to light that the orientation of the whole animal in space depends essentially upon this sensitiveness. This fact may perhaps explain why Branchiomma, a Serpulida quite similar in structure to Spirographis, has well-developed eyes upon its gills.

9. If Spirographis is carefully removed from its tube, it is not able to raise its body from the floor. In such a condition it creeps about like an earthworm, only much more slowly. I have occasionally seen such animals creep to the window side of the aquarium. They appeared, however, to suffer from contact stimuli, to which they were constantly exposed in this condition; they all died within a few days.

10. I am not in a position to make a definite statement concerning the mechanics by which the heliotropic curvature of the tube is brought about in these animals. The wall of the tube of an adult animal is $1.25-1.5 \mathrm{~mm}$. or more thick. It is very flexible and elastic. If the animal is taken out of the tube after the latter has been bent through the heliotropic reactions of the animal, the tube nevertheless maintains its 
curvature. The wall on the outer concave side of the tube is therefore permanently shortened. It might seem that the limit of elasticity of the tube is so low that it retains, like a piece of lead, a curvature imparted to it through the muscular force of the animal. But this is not the case. I put a thick rod of lead into the straight tube of a Spirographis and bent it till the tube was strongly curved. The lead rod was allowed to remain in the tube. When a week afterward I withdrew the rod from the tube, it retained only a trace of the curve impressed upon it. Similar failure followed my attempt to straighten by the same method, a heliotropically curved tube. Yet, as I have already shown, Spirographis is able to straighten its curved tube within a few hours after a change in the direction of the rays of light, and, what is more, the tube remains straight. The tube retains its curvature even after it has been split open. The animal has, however, besides pressure and pull, another means at its disposal to change permanently the orientation of the tube, namely, the production of a secretion and the formation of a new layer within the tube. The idea that permanence in the curvature is attained in this way is supported by the fact that the inner layer of the tube is much more elastic than the outer layers, so that the formation of a new inner layer on one side of the tube might curve it permanently. The following fact supports this view: If a tube is cut open lengthwise, the cut margins roll inward. If the individual layers are separated, as can be done easily, it is seen that the tendency of the inner layers to curl up is greater than that of the outer layers, and that of the innermost, newest layer is the greatest of all. The formation of a new inner layer on one side of the tube would, therefore, be sufficient to maintain the curvature of the tube permanently. The formation of a new layer cannot be observed directly. One is also disappointed in the hope of finding one side of the wall of the 
tube thicker than the other, for the thickness of the wall of perfectly straight tubes varies greatly in different places of the same cross-section. The thickness of the wall is therefore no criterion in answering our question. I can therefore formulate the following theory of the origin of the heliotropic

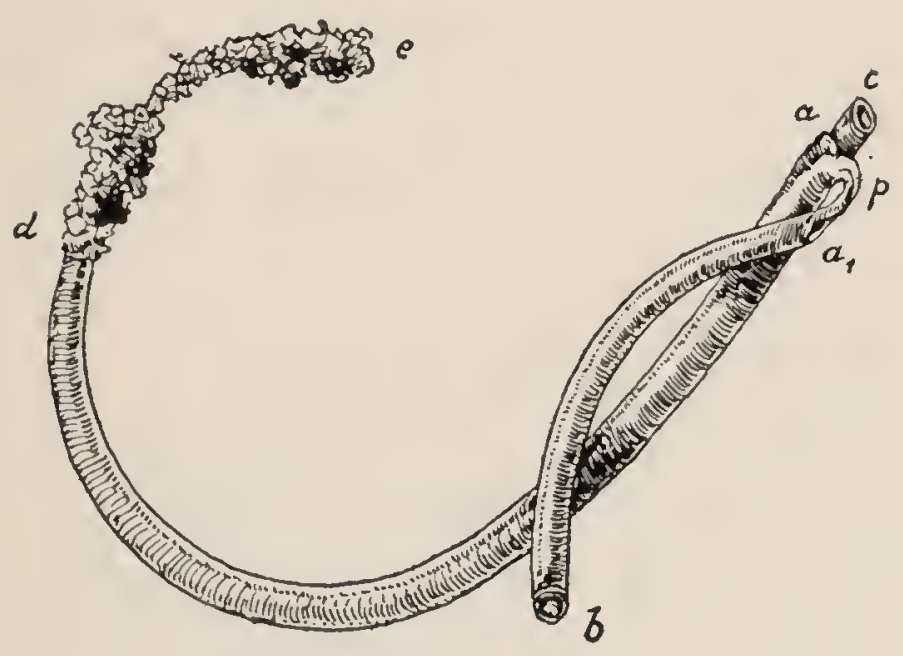

FIG. 11 curvature in the tube, only by reserving the right to test, and perhaps modify it later. I believe that, when illuminated from one side only, the animal strives at first to bring the axis of its gills as nearly as possible in the direction of the rays of light. In doing so the animal perhaps bends the tube by aid of its muscular force. Since the tube, however, tends to resume its original position because of its elasticity, the body of the Spirographis must rub more strongly against the concave wall of the tube than against the other. This increased friction brings about a great activity of the skin glands, whose secretion forms the material of the tube. That friction indeed leads to secretion, and with it to the formation of a tube, I have been able to prove directly in the case of the Actinian, Cerianthus membranaceus. I have been able to establish the following facts regarding Spirographis which seem to indicate a similar behavior. I cut small pieces from the tube. The animal was in consequence obliged to rub against the cut margins during its movements; and a copious secretion was indeed formed in a short time, which soon closed the opening with a new membrane. There is, moreover, always more or less friction on the anterior margin of the tube when the animal stretches out its head. In fact, the tube grows constantly from this 
end, as illustrated in Fig. 11. In this experiment I had cut a long broad piece $a a_{1}$ out of the tube at $a$, so that the anterior piece of the tube $a_{1} b$ remained attached to the rest of the tube only by a thin piece $p$. After the operation the animal showed its gills at $a$, and no longer used the piece $a_{1} b$ of the tube. New material was deposited at $a$ within a few days, and in the course of three weeks the new piece ac was formed. Its light color readily characterized it as new. I had at the same time cut away the aboral end of the tube completely. Before my very eyes the movement of the aboral end upon the sand caused the secretion of a sticky mass, to which particles of sand became attached. In this manner the new piece of tube de was built, consisting of grains of sand cemented together by the glandular secretion from the tube: The newly formed piece was perfectly smooth on the inside. The secretion from the skin glands continues as long as there is any noticeable amount of friction. When I removed Spirographis from its tube and placed it in a smooth test-tube, practically no secretion occurred. Secretion occurred only from the parapodia in the form of long, fine threads, similar to those produced by the spinning glands of spiders. If, however, the naked Spirographis was laid upon the sand, the aboral end was soon covered by a shell of sand kernels. I have never, however, seen the animals form a complete tube when removed from their old ones; for in their exposed condition they soon die.

\section{II}

Spirographis Spallanzanii attains its heliotropic orientation when illuminated from one side by curving its flexible tube; new growth of the tube is not necessary. There are other Serpulidæ, however, the calcareous tubes of which are stiff and inflexible. These Serpulidæ, like Spirographis, expose their gills to the light, and these, too, react according 
Further Investigations on Heliotropism 101

to their structure as radial organs. Such a Serpulida, if heliotropically irritable, must place the longitudinal axis of its cylindrical tube in the direction of the rays of light. If the calcareous tube is brought into any other position with reference to the source of light, the animal must make use of one of two possibilities in order to regain its proper orientation: either it must lengthen its calcareous tube and bend the newly growing part until the axis of the tube again

$$
a \leftarrow-b
$$

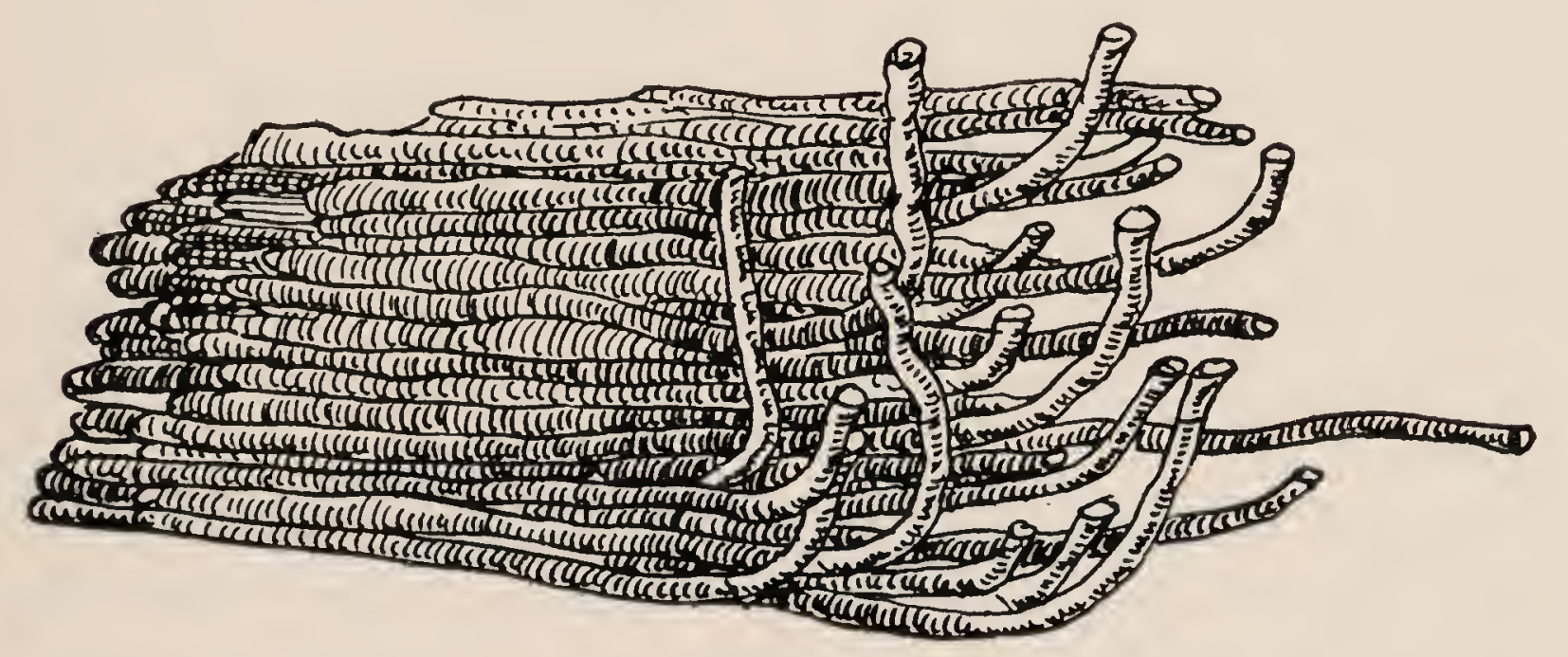

FIG. 12

lies in the direction of the rays of light, or else leave its tube entirely and build a new one having the proper orientation. The animal makes use of the first of these possibilities. I experimented with Serpula uncinata. These Annelids inhabit calcareous tubes and are gregarious. Large white blocks are found in the Gulf of Naples which consist entirely of the tubes of countless numbers of such Annelids massed together. I noticed that the individual tubes in such a mass all had the same orientation, and in those cases in which the blocks showed the base upon which they had rested on the horizontal bottom of the ocean it was plainly visible that the tubes must have stood in the water with their longitudinal axes vertical. Serpula can, like Spirographis, move about freely within its tube. I laid a large block of innumerable annelid tubes, each of which 
stood almost mathematically straight and parallel, upon the floor of the aquarium so that the longitudinal axes $a b$ of the tubes, which had previously been vertical, now had a horizontal position (Fig. 12). The light fell into the aquarium from above. I noticed that in the course of the next day the Serpulidæ, which like Spirographis presented only their radially arranged gills to the light, bent them strongly upward. Individual tubes then began to grow, and in such a way that the newly formed portions of the tubes all bent upward until the free tip of the tube lay in the direction of the rays of light (which in this case was identical with the direction of gravity), after which the tubes continued to grow in the direction of the rays of light (and of gravity). Within six weeks the entire block was covered with tubes which curved upward; not a single individual had continued to grow in the original direction $a b$. The figure shows the Serpulidæ curving upward at the free edge of the block. The final effect in this case therefore again corresponds to the theory of geotropism and heliotropism as presented by Sachs: the axis of the gills which react as a radial organ lies finally in the direction of the rays of light (and of gravity). While in the case of Spirographis, however (the tube of which is flexible), this effect was brought about through a change in the orientation of the old tube, the same effect was attained in the case of Serpula (the tube of which is inflexible) only through the heliotropic curvature of that portion of the tube which was in the process of growth.

In the above-mentioned experiment the direction of the light rays was identical with the direction of gravity. I have not yet been able to decide whether light alone determines the orientation of the tube, or whether gravity also plays a rôle. I hope later to make a series of experiments regarding this point. 


\section{III}

1. I have endeavored to find other animals in which heliotropic curvatures are formed only in the growing parts. These efforts have been successful in the Hydroids. Stems of Sertularia (polyzonias?) were cut off near the root and fixed in the sand in an inverted position, so that the cut end was directed upward. The stems were placed near a window through which the light fell obliquely and from above. The animals began to regenerate; new polyp-bearing stems grew from the cut end as well as new roots; ${ }^{1}$ but while the new stems grew upward and toward the window, the roots grew downward and toward the room side. The polyp-bearing shoots are positively, the roots negatively, heliotropic. That the negatively heliotropic elements were true roots was proved by the fact that when brought in contact with a solid body they attached themselves to it and continued to grow over its surface in close contact with it. They could be loosened from their attachments only by force. The polypbearing stems do not possess this kind of contact-irritability. The heliotropic phenomena will be readily understood by the aid of Figs. 13, 14, and 15: $a b$ is the old stem, $b$ the cut end the stem is fixed in the sand to the point ac. From the cut end $b$ arise newly formed roots $W_{1}$, which bend downward away from the light and toward the room side of the aquarium. The new polyp-bearing shoots $S$ grow upward and toward the window. The arrow marks the direction of the rays of diffuse daylight in this experiment.

2. In these experiments new growths occasionally sprang from the middle of the old stem, which, so far as their contact irritability was concerned, reacted as roots. Those tencirils which attached themselves to solid bodies were always negatively heliotropic. They grew downward and

1 Which is of importance in the theory of organization. 
toward the room side, and remained free of Hydranths. (See Fig. 13, $W_{2}$.)

On the other hand, I saw also new polyp-bearing stems arise from the old stems, although much less frequently; these grew in the opposite direction, namely upward.

3. That in the case of Sertularia it is, indeed, only the growing parts which produce the heliotropic curvatures is

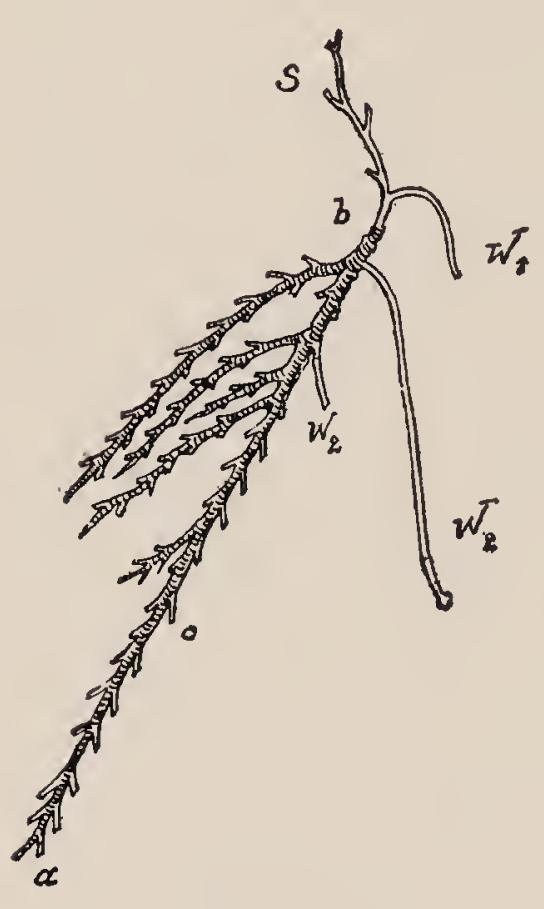

FIG. 13

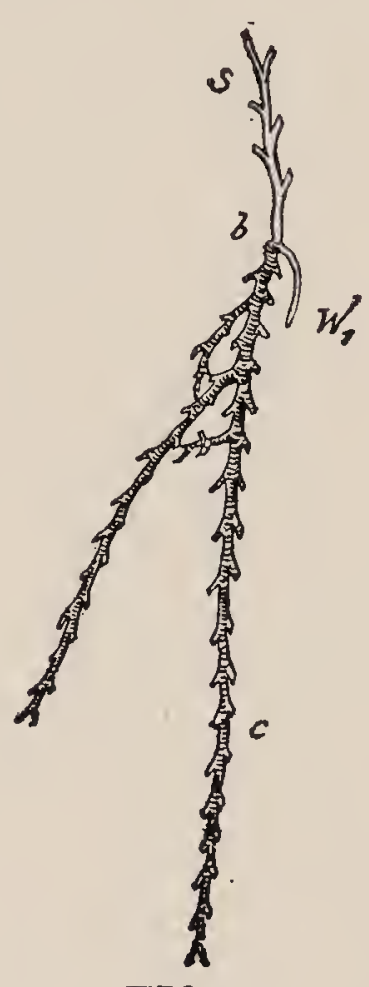

FIG. 14

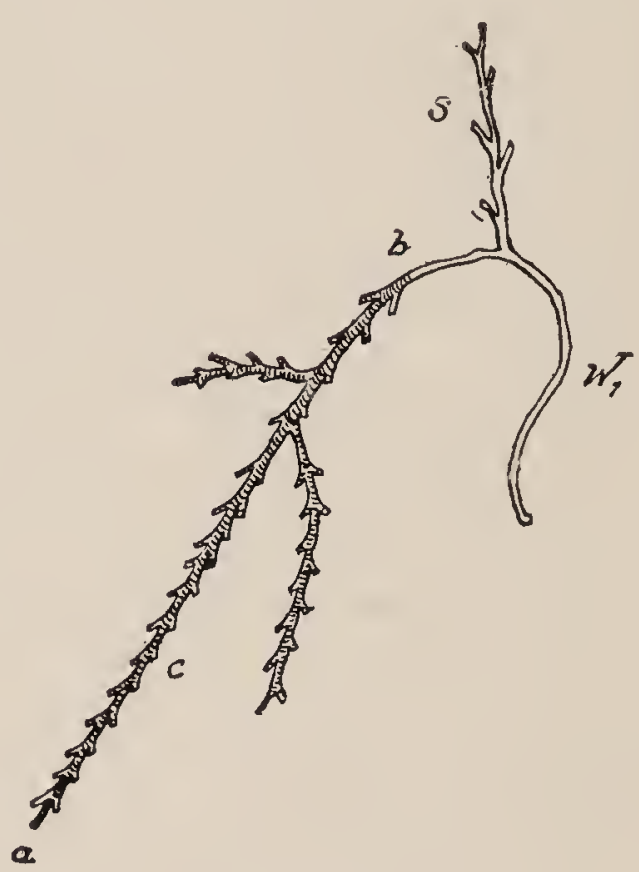

FIG. 15

shown by the following experiment. The growing tips were cut off a large number of Sertularia stems. The stems began to grow, and in the course of a few days sent out new sprouts. The new growth is strikingly different in color from the old stem; while the latter is rather brown (from having been covered by Algæ?), the color of the new growths is a light yellow. The growing elements curved themselves until the growing points lay in the direction of the rays of light and then continued to grow in this direction. During all this time no change in the orientation of the old stem occurred, nor did any take place in other uninjured stems, in which no linear growth occurred during this time.

How far gravity played a rôle in these experiments I was 
unable to determine accurately. The Sertularia cultivated in a dark room ceased to grow, though I question whether this was entirely due to lack of light.

4. Light (and perhaps gravity) influences not only the orientation, but also the position of the newly formed organs. I have observed, and not in the case of Sertularia only, that the new polyp-bearing branches always arise from the upper surface of the stem. In Fig. 15, a new stem $S$ springs from the upper side (the side directed toward the source of light) of the stolon $W_{1}$. I do not desire to discuss these points more minutely here, as they will form the basis of a paper which is to appear soon, on the form of animals.

The experiments on Sertularia described here serve only to complete the general consideration of animal heliotropism and to show more fully the identity of animal and plant heliotropism. The special investigation of the heliotropic behavior of Hydroids is to be the subject of future study. That this is both an interesting and a fruitful field is shown by the beautiful work of Hans Driesch; which has just appeared, on the "Heliotropism of Hydroids." I Driesch arrives at the following result:

The stolons which are produced instead of polyps under unfavorable conditions in Sertularella polyzonias, are with the exception of the first, which is turned away from the light from the very beginning, all positively heliotropic at first, becoming negatively heliotropic after the growth of the daughter-stolons. They arise from the side of the mother-stolon, which is turned toward the light. (P. 152.)

This observation of Driesch agrees very well with mine. I shall return to them in my "Physiological Morphology of Animals."

The results of this study may be summarized as follows:

1. Certain sessile animals (Serpulidæ, Hydroids) which are compelled to react to light and gravity as radial organ-

1 Zoologische Jahrbücher, Vol. v. 
isms always place the axes of their radial organs in the direction of the light rays, as do the radial organs of sessile plants.

2. The fact that sessile animals, such as the Serpulidæ, have a central nervous system, while plants have not, does not bring about any difference in the heliotropic effect.

3. If the light enters from one side, there are produced in the above-mentioned animals heliotropic curvatures which correspond to those obtained in sessile plant organs under similar conditions.

4. There are sessile animals which attain these heliotropic curvatures only during the period of growth, as is the case with certain plants. Sertularia and Eudendrium, among others, belong in this group, in which only the growing parts are able to bend heliotropically; Serpula uncinata, which is able to change the orientation of its otherwise stiff tube only when the latter is growing, also belongs in this group.

5. Spirographis Spallanzanii, the tube of which is flexible, is capable of heliotropic curvatures without accompanying phenomena of growth, as are also certain jointed plant organs which attain their heliotropic orientation without phenomena of growth.

Although I do not consider my study of animal heliotropism ended with this paper, yet I think I have shown that the heliotropism of sessile animals is essentially identical with the heliotropism of sessile plants. 


\section{III}

\section{ON INSTINCT AND WILL IN ANIMALS ${ }^{1}$}

1. In the biological literature one still finds authors who treat the "instinct" or the "will" of animals as a circumstance which determines motions, so that the scientist who enters the region of animated nature encounters an entirely new category of causes, such as are said continually to produce before our eyes great effects, without it being possible for an engineer ever to make use of these causes in the physical world. "Instinct" and "will" in animals, as causes which determine movements, stand upon the same plane as the supernatural powers of theologians, which are also said to determine motions, but upon which an engineer could not well rely.

My investigations on the heliotropism of animals led me to analyze in a few cases the conditions which determine the apparently accidental direction of animal movements which, according to traditional notions, are called voluntary or instinctive. Wherever I have thus far investigated the cause of such "voluntary" or "instinctive" movements in animals, I have without exception discovered such circumstances at work as are known in inanimate nature as determining movements. By the help of these causes it is possible to control the "voluntary" movements of a living animal just as securely and unequivocally as the engineer has been able to control the movements in inanimate nature. What has been taken for the effect of "will" or "instinct" is in reality the effect of light, of gravity, of friction, of chemical forces, etc. The following may be added by way of fuller explanation: 
The position which the tube of Spirographis Spallanzanii assumes in space is such, as we have seen, that the animal turns its oral pole toward the light, and puts the axis of its radial gills into the direction of the rays of light. The direction of the rays of light is the condition which determines the orientation of these animals unequivocally. If the question should arise as to how to hold a great number of living Spirographes continually and voluntarily in a definite position in space, this could be done, as our investigations have shown, by simply allowing the rays of light to fall upon the animals in the direction which we wish the animals to assume and hold. If anyone endeavors to compel Spirographis to assume a definite spatial orientation either through "instinct" or "will," he will be obliged to seek the aid of the rays of light in order to obtain the desired result, even if he afterward believes that, beside, before, behind, after, or between the light rays the "instinct" or "will" of the animal co-operated with the light to bring about the movement. He will further be able to convince himself that the direction of the light, if sufficiently intense, is alone and unequivocally able to determine the orientation.

The direction of the "voluntary" movements of the winged plant lice is determined by the direction of the rays of light. The animals are forced to turn their oral poles toward the light and to move in the direction of the rays of light. If the animals are introduced into a transparent vessel, they live and die on the side of the vessel which is turned toward the light. If anyone should wish to force these animals to move in a fixed direction toward a definite point "voluntarily," he knows now how this may be accomplished. He need only allow sufficiently intense light to fall upon the animals in the direction in which it is wished that they should go.

As is well known, the direction of the rays of light, par- 
ticularly that of the more refrangible ones, determines also the orientation of the organs of a plant. By the help of light the botanist controls the orientation of a plant at will. Why should he maintain that the "will" or the "instinct" of the plant co-operates with the rays of light when the orientation is determined solely and unequivocally by the latter? The movements of an animal toward the light are, however, as I have shown, identical point for point with the movement of a plant toward the light. Wherever the orientation of plants has been satisfactorily controlled experimentally, light has, indeed, been considered the sole determining factor ; but in the case of animals, in which in similar experiments light is without doubt also the sole determining factor, "instinct" and "free will" have still been considered to play a rôle.

Just as the direction of the rays of light (particularly that of the more refrangible ones) is the essential factor in the examples described above, and in many others given in my papers on heliotropism, so in other cases it is gravity, in others again contact with solid bodies, in still others chemical forces, etc., which determine the movements of the animals.

2. In order to state the cause which determines in each instance the "voluntary" movements of an animal, I designated the movements by their external cause. I spoke therefore, as has long been the custom in plant physiology, of heliotropism when the direction of the rays of light determines the direction of the movements of an animal or its orientation; of geotropism, when gravity, or of stereotropism, when contact with solid bodies, determines the orientation or the movements; etc.

A zoölogist asked me reproachfully what had been gained by designating as "stereotropism" what had been designated as "instinct." I was discussing the fact that certain animals creep into the crevices of solid bodies, and the zoölogist 
was of the opinion that the animal behaves thus through "instinct." If a physicist finds that liquids rise in a capillary, or that one liquid forms a convex while another a concave, meniscus in a glass tube, he will be less easily satisfied than the zoölogist, according to whom everything is done through "instinct." The physicist will endeavor to discover more precisely what conditions underlie the phenomenon. This, it seems to me, is also the problem of the biologist - a problem which is not even recognized, much less solved, by saying the cause of such or such a motion is an "instinct." From a biological standpoint one would at first take it for granted that light causes animals to creep into crevices. But I was able to show that the animals creep into the crevices between solid bodies even when the solid bodies are perfectly transparent and are exposed to a strong light; secondly, that the animals behave in a similar way when put in a perfectly dark room. Light is not, therefore, the physical cause which determines this phenomenon. I proved this for Forficula, ants, the larvæ of Musca vomitoria, etc. Plateau had previously established this fact by a similar experiment upon Cryptops, with which I was not familiar at that time, however. The animals creep into narrow crevices, therefore, not because of the light, but because they are forced to bring as much of their bodies as possible in contact with solid bodies. The friction and the pressure produced by the solid bodies are therefore the determining cause. This view, that light has nothing to do with the phenomenon, but that it is the friction produced by contact with solid bodies, has this advantage over the traditional phrase "It is instinct," that pressure and friction are physical agencies which, like light, can be controlled quantitatively and qualitatively, and by which we can prescribe unequivocally the "voluntary" movements and the "voluntary" orientation of an animal.

I will here add that, while there are a large number of 
animals which are forced to bring their bodies in contact with solid objects on all sides as far as possible, there are others which show exactly the opposite form of irritability and immediately draw themselves away from a solid body with which they chance to have come in contact. To these belong the Nauplii of Balanus perforatus, the tiny Mysidex of the Bay of Naples, the gills of Spirographis Spallanzanii, etc. That that form of irritability which I have called "stereotropism" plays a prominent rôle in lifephenomena, however, follows from the fact that the entrance of the spermatozoon into the egg (as shown by the investigations of Dewitz ${ }^{1}$ ) is governed by this form of irritability, and that the migration of leucocytes is likewise determined largely by contact-irritability. I have, moreover, incidentally found, in my investigations on the influence of external stimuli upon the form of the body, that stereotropism influences not only the shape, but also the size and velocity, of the growth of certain organs. These investigations were made upon Hydroids. I succeeded in producing stereotropic curvatures (away from solid bodies) in certain organs with the same certainty that I produced heliotropic curvatures. Certain organs, when not in contact with solid bodies, attain, within the same period of time and under otherwise similar conditions, only one-tenth the length which they attain when in contact on one side with a solid body. It is for these reasons that I have made no mistake and performed no useless task in calling attention to the importance of this contact-irritability in the animal kingdom, to which I have found it necessary to give a special name.

3. I have thus far given only examples in which a single source of stimulation determines the "voluntary" movements of animals. But in a large number of cases the movements of animals are not dependent upon one cause of stimulation

IDewrtz, Pflügers Archiv, Vol. XXXVII. See also MAssart, Bulletin de l'Académie royale de Belgique (Bruxelles, 1888). 
alone; more frequently several causes co-operate, and the movements which are produced in this way, the cause of which is again sought by many in the "will" of the animal, are only the resultant of various causes operating at the same time. In very intense light the full-grown larvæ of Musca vomitoria move away from the light in the direction of the light rays: they pass by a piece of meat lying in their way. If the light is sufficiently weak, however, the chemical influence of the volatile substances arising from the meat exceeds the orienting influences of the light and the larvæ crawl to the meat. With other animals which are still more susceptible to chemical stimuli-as, for example, the male Lepidoptera, which, as is well known, are attracted to the female from great distances entirely through the effect of chemical stimuli-heliotropism may be entirely masked by these chemical stimuli. It is not always an easy matter to say, from the movements which an animal always executes, what are the conditions determining these movements.

4. Another complicating circumstance is still to be added. Life-phenomena are phenomena of irritability; $i . e$. , they are not dependent solely upon the external causes acting upon the organism at a given moment, but upon these and the conditions present within the organism taken together; and the latter conditions are in themselves variable. The study of animal heliotropism revealed the fact that one and the same animal may react differently toward the light during different periods of its existence. The caterpillars of Porthesia chrysorrhœa after having fasted through the winter are energetically positively heliotropic. After the animals have eaten, heliotropism may still exist, but intense light, which formerly determined their movements with definiteness, has no more effect upon them than did quite weak light previously. Plant lice become sensitive to light - that is to say, positively heliotropic-only after they have fed; the 
larvie of Musca vomitoria are energetically negatively heliotropic only when fully grown, etc. In ants sensitiveness to light is, as I have shown, connected with sexuality. The males are more sensitive than the females; at the time of the nuptial flight the males and females become energetically heliotropic, while the so-called workers remain practically uninfluenced by the light. There must also be mentioned the change which occurs in the sense of heliotropism of many animals in different stages of their development. The fullgrown larva of Musca vomitoria is negatively heliotropic; yet the sexually mature insect is positively heliotropic. Such a behavior is quite widely distributed.

Finally, it is not infrequently possible to change at will, through the influence of light, positively heliotropic animals to negatively heliotropic animals, and the reverse. The larvæ of Balanus perforatus, the larvæ of certain worms, and indeed a large number of other animals, become positively heliotropic when they are left in the dark for a long time. If they are brought into light of sufficient intensity, they become negatively heliotropic after a time, and this the more quickly the more intense the light.

We do not, therefore, always meet with simple conditions in analyzing the causes which determine the "voluntary" movements of an animal; but, however complicated they may be, the "voluntary" movements of animals are nevertheless, as our experience indicates, always unequivocally determined only by such circumstances as determine also the movements of bodies in inanimate nature.

5. To be sure, many of the authors who oppose my con clusions would protest if it were said of them that they hold the "will" to be something which cannot be explained on physical or chemical grounds. But if some physical agency is pointed out which prescribes unequivocally the orientation of an animal body or the direction of its movements, which 
were formerly believed to be determined by the "will" of the animal, the authors are still dissatisfied. They did not doubt that ultimately a physical solution of the question would be found, but they expected something more sublime, something which is more closely related to the mysticism of the ganglion cells. Of course, our knowledge of the process is not exhausted when it is proved that the direction of the rays of light prescribes the direction of the progressive movements of Hrmatococcus swarm-spores or the Nauplii of Balanus; just as little as the knowledge of the chemical effects of light is today exhausted. Yet no one will say that "instinct" is the determining circumstance in these physical phenomena.

6. Just as the past generation of physiologists felt it to be a handicap that instead of looking for the causes of life-phenomena, investigators were satisfied with the phrase, "The vital force is the cause," so it is a handicap to us that within the more limited sphere of the so-called psychic lifephenomena the influence of this scholastic method of thinking has survived to the present time. The handicap lies in the fact, that if one says that "instinct" or "will" determines a motion, the true problem involved is ignored or concealed. This true problem is the analysis of the circumstances which in each case determine unequivocally the "voluntary" movements of an animal. It was the object of this paper to point out that we must endeavor to solve this problem with as little concern for "instinct" and "will" as for "vital force." 


\section{IV}

\section{HETEROMORPHOSIS ${ }^{1}$}

\section{INTRODUCTION}

IT is well known that a number of animals possess the power of forming a new organ in the place of an organ which has been lost. It has always been taken as a matter of course in animal physiology that the regenerated organ is necessarily identical in form and function with the one which has been lost. The experience of the botanists, however, shows that this does not hold true in the case of plants, and a few sporadic observations upon animals - which, however, have not been taken into consideration for this problem - seemed to suggest that similar conditions might be found in animals. I have undertaken the task of finding out whether and by what means it is possible in animals to produce at will in the place of a lost organ a typically different one-different not only in form, but also in function. It is my purpose to report the results of these experiments in the following pages.

The organs which I tried to substitute for each other in these experiments are the oral and aboral poles (head and foot). I have succeeded in finding animals in which it is possible to produce at desire a head in place of a foot at the aboral end, without injuring the vitality of the animal. The animal shown in Fig. 16, a Tubularian, has by artificial means been so altered that it terminates in a head at both its oral and aboral ends. If, for any reason, it were necessary to create any number of such bioral Tubularians, this demand could be satisfied. In another Hydroid, Aglaophenia pluma,

1 Wärzburg, 1891. The pamphlet is dated 1991, although it appeared in 1830. 
it is possible so to change the form of the animal that it terminates at both ends either in oral (Fig. 17) or aboral poles, and yet continues to live. On the other hand, I have found animals in which all attempts at the transformation of organs have thus far been unsuccessful. To this group belong Cerianthus membranaceus and many other Actinians. I succeeded, however, in bringing about a permanent change of form in one of these animals (Cerianthus membranaceus), in which I was able to cause the growth of any number (within certain limits) of mouths, one above the other, in one and the same animal.

The regeneration of lost organs in animals has often been made the subject of study, usually, however, only to see which organs can be regenerated, and further to study more closely the anatomical or histological details of the process of regeneration. But it has rarely been considered that these phenomena can give us an insight into the conditions that control the morphogenesis of animals. Where this has been done, it has almost always been with the intention of showing that under all conditions only one and the same organ grows from any definite point on the animal.

Allman ${ }^{1}$ was perhaps the first to define this sharply as a law of the formation of organs. From the well-known experiments of Trembley, ${ }^{2}$ Dalyell, ${ }^{3}$ and from his own observations, he formulated the theory of the "polarity" of the animal body. Allman cut pieces from the stem of Tubularians and marked the end which had been directed toward the head of the animal. Even though this cut end was morphologically entirely similar to the other, yet a head was formed only at this oral end, while no head was formed at

\footnotetext{
1 George J. Allman, Report of the British Association for the Advancement of Science, 1864.

2 A. Tremblex, Mémoires pour servir d̀ l'histoire d'un genre de polypes d'eau douce d̀ bras cn forme de cones (Leide, 1744).

3 J. G. DALYeli, Rare and Remarkable Animals of Scotland (London, 1847).
} 
the opposite end. That Allman chose the name "polarity" for this behavior suggests the possibility that he may have thought of the analogy of this fact to the behavior of a magnet; for a fragment of a broken magnet always has a north pole at that end which in the original magnet was directed toward the North Pole. If however, the book of Dalyell is subjected to a close scrutiny, it is found that this author occasionally (at two or three places in the book) mentions observations which do not harmonize with the theory of polarity. In these cases, however, Dalyell believed that he was dealing with accidental monstrosities which this careful and patient observer did not consider of sufficient importance to follow out experimentally, or to take into consideration for a theory of organization.

W. Marshall ${ }^{1}$ builds on Allman's theory of polarity in his experiments upon Hydra. When Hydra vulgaris is cut into pieces, "one is struck most forcibly with the extraordinary polarity of the animal, in consequence of which new tentacles and a new mouth are always formed at the oral edge of the cut piece" (p. 698).

A further expression of this idea is found in Nussbaum's papers on "The Divisibility of Living Matter."' Nussbaum found that when a piece is cut from an Infusorian, new cilia develop from the edge of the wound in the same number and in the same position that they occupied before the injury. He goes even farther than Allman and concludes that

Every minute particle of living protoplasm is oriented; otherwise we could not understand the regular appearance of new cilia at definite points when the infusorian bas been divided. Just as we can distinguish in an infusorian between the anterior and the posterior, right and left, and dorsal and ventral surfaces, so each minute particle of protoplasm must likewise be oriented according to the three axes in space.

1 W. Marshald, Zeitschrift für wissenschaftliche Zoologie, Vol. XxXVII (1882).

2 M. NUssbauM, Archiv für mikroscopische Anatomie, Vols. XXVI and XXIX. 
Almost all the numerous other authors who have worked upon the regeneration of organs in animals also regard it as self-evident that the regenerated organ must be identical with the lost organ in form and function. The facts which I shall bring forward in the following pages will show, however, that this theory is certainly too narrow. For I succeeded in doing away with "polarity" first of all in that very animal upon which Allman based his theory of "polarity"—namely, in Tubularia.

One of the first authors who concerned himself with the study of the phenomena of regeneration, Charles Bonnet, looked upon them in a less biased way than did Allman. Bonnet, to whom Trembley had very early communicated the fact of the phenomenal regenerating power in Hydra, attempted to convince himself of the truth of Trembley's statements; since, however, he was unable to obtain Hydra, he tried whether similar results could not be obtained upon worms. Bonnet used two species of worms in his experiments. In the first species, which he designates as vers rougeâtres, he found the conditions which are typical for Hydra, and which correspond to the theory of "polarity." If the head of such a worm was cut off, a new head was formed at the cut end; when the tail was cut off, a new tail was formed at the point of section. If the head and tail were both cut off, a head was formed at the oral end, and a tail at the aboral end. In a second species, the vers blanchâtres, the results were not so regular. When only the head or tail was cut off, the lost part was always regenerated. If, however, a piece was cut out of the middle of the worm, it happened that such a piece formed a tail at the oral end, instead of a head. Bonnet observed this three times. ${ }^{1}$

I have found no reference in the literature which would indicate that these observations of Bonnet have ever been

1 CH. Bonnet, Euvres d'histoire naturelle et de philosophie (Neuchâtel, 1779), Vol. I (Traité d'insectologie). 
repeated and confirmed. I am not in a position to state whether they are correct or not.

The theory given by Bonnet is in some points similar to a theory brought forward by Duhamel in his Physique des arbres, and to which Sachs goes back in his papers on "Stoff und Form der Pflanzenorgane."

Bonnet believes that just as there are specific germs for the development of the entire animal, there are also special germs for the development of the various organs; he assumes the existence of certain head germs and certain tail germs. In order, however, that these germs may develop, they must be particularly well nourished. Their nutrition is accomplished, as in plants (according to Duhamel), by various kinds of saps, one of which serves for the nutrition of the head, while the other nourishes the tail. The latter flows from head to tail, the former in the reverse direction. If, now, the head is cut off, the saps which heretofore served to nourish the head, can now be utilized for the nutrition of the head germs, and the latter begin to grow out at the cut oral end into a new head. In a similar way the tail germs may begin to grow when the tail is cut off. It is assumed that the tail germs and the head germs are distributed evenly throughout the body of the vers rougeatres; for this reason a head must always grow from the oral end of a fragment cut from any portion of the animal, while the aboral end must always give rise to a tail. Upon the other hand, in the vers blanchatres the head germs are found only in the neighborhood of the head, while the tail germs are distributed through the entire body. For this reason the worm regenerates a new head when the head is cut off, while a new tail is formed at either end when a piece is cut out of the middle of the worm. ${ }^{2}$

1 Arbeiten des botanischen Instituls in Würzburg, herausgegeben von SACHS, Vol. II (1882), pp. 452 and 689.

2 CH. Bonvet, Considération sur les corps organisés, Art. 259 ff.; QEurres (Neuchâtel, 1779), Vol. VI, pp. 48 ff. 
I shall not discuss the importance of the theory of Bonnet. I only mention it here because it takes into consideration the fact that sometimes a tail may be formed instead of a head, which is not done in Allman's theory of polarity. I shall avoid all theoretical discussions in this paper, and con. fine myself to the task of showing whether and how it is possible to cause with certainty in an animal the growth of an aboral pole in the place of an oral one, and vice versa, at will.

For the formation of an organ which in form and function is different from that which has been lost I shall use the term heteromorphosis. By the term regeneration I understand the replacement of a lost organ by one which is identical with that which has been lost.

\section{HETEROMORPHOSIS IN TUBULARIA MESEMBRYANTHEMUM}

A layman would be in doubt as to whether he should call a specimen of Tubularia mesembryanthemum a plant or an animal. From a much-branched system of roots (or stolons), which are attached to a solid substratum, arise numerous delicate unbranched stems, several centimeters high, which end in polyps that are usually red and look very much like flowers. These polyps take up and digest the food for the animal. The animals belong to the class of Hydroids and are found in great numbers in the Bay of Naples.

The zoölogists have developed a very complicated terminology for the individual organs of the Hydroids, which may be very useful in purely descriptive morphology, but does not take into consideration the forms of irritability of the various organs. Causal morphology, which attempts to discover the circumstances that determine form, has to consider first of all the irritabilities of the individual organs. For the purposes of the physiologist it is therefore necessary to take these into account in describing and naming the various organs. 
I distinguish in Tubularia, according to the differences in irritability, between the stems and the root. By the root is understood that part of the Tubularian which is endowed with a special contact-irritability (stereotropism), by virtue of which it attaches itself to solid bodies and keeps the animal in a fixed position. By the stem is understood that part of the animal which bears the sexual elements and the polyps, and which is endowed with the opposite irritability, in consequence of which it grows away from the substratum to which the animal is attached. This simple terminology, which is based upon the irritability of the organs, will suffice for our purposes. Of the entire animal only the polyps can move spontaneously; the stem is immovable. If we cut a piece out of a stem, we must discriminate between its oral and aboral ends, according to the orientation of the piece in the original uninjured animal. The oral end is that which was originally directed toward the polyps, the aboral end, that which was directed toward the root. I shall now describe the main experiments individually.

1. I cut off the roots and polyps of a series of stems, and put these mutilated stems with their aboral ends vertically into the sand sufficiently deep to keep them in a vertical position. At the free oral ends, which were surrounded on all sides by sea-water, new polyps were formed in a short time-at the proper temperature and with favorable specimens within two days. These corresponded in form with the old polyps. No growth took place at the ends which were buried in the sand, no matter how long the observations were carried on (in some instances for several months).

When I put stems with their oral ends in the sand, $a$ polyp was formed at the free, aboral pole. In favorable cases this was formed in a few days. Neither a polyp nor a root was formed at the oral end, which had been covered by sand, no matter how long I continued my observations. 
Contrary to the theory of the "polarity" of the animal body, therefore, fragments of Tubularia mesembryanthemum are able to form polyps even at their aboral ends.

2. I supported pieces cut from the stem of Tubularia mesembryanthemum in such a way that both cut ends were

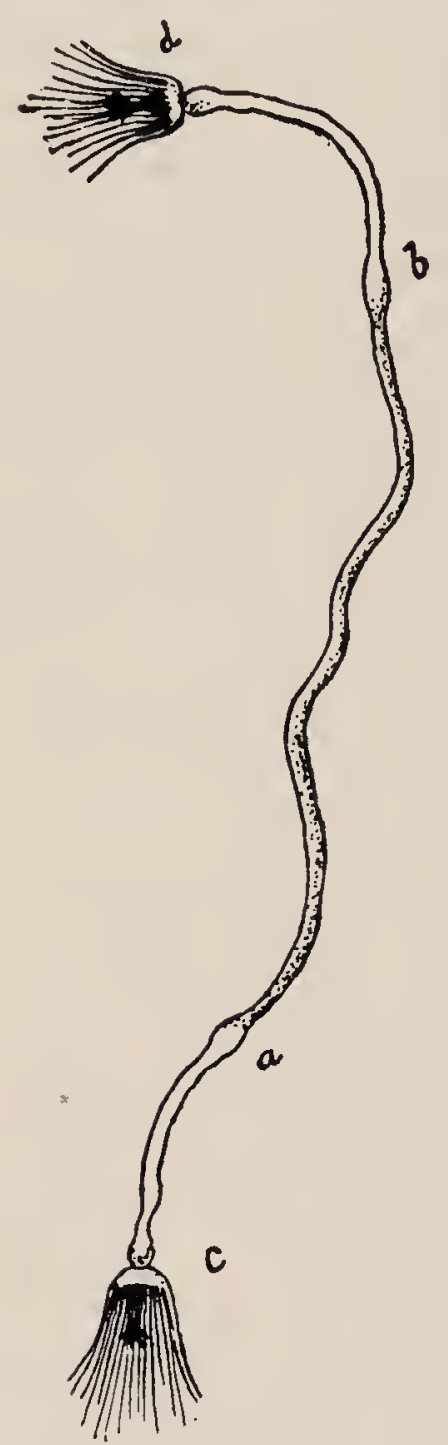

FIG. 16 surrounded by water. To do this I supported them in the meshes of a long wire net, or in the holes of a metal plate set up in the aquarium for this purpose. Polyps were formed at both the oral and the aboral ends of the fragments, so that the stem terminated in a head at each end. Fig. 16 represents such an animal sketched from life and enlarged twice. $a b$ is the piece removed from the old Tubularian. Polyps were formed at both ends, and the stem then grew in length from both ends. ac and $b d$ are the new pieces that grew after the formation of the polyps.

I have in this way been able to produce at any time any number of animals which terminate in an oral pole at each of the two ends of their body. I shall hereafter designate animals which terminate in a head at each end bioral animals.

I would particularly emphasize the fact that such an animal remains bioral for the rest of its life. It is a well-known fact that in a normal animal the oral polyp is lost spontaneously after some time, and that a new one is formed sooner or later in its place. In the case of the bioral animals a constant blooming, shedding, and reappearance of the polyps occurs, not only at the oral end, but also at the aboral end, during the entire duration of their life.

3. I was able, therefore, not only to cause the develop- 
ment of a head at both ends of the fragment of a stem, but also to prevent the formation of a head at will, by simply putting this pole into the sand. When both poles are put in the sand, no head is formed at either end. If one of the poles which has been in the sand for some time, and on which the formation of a head has been prevented in this way, is pulled out of the sand so that it is again surrounded on all sides by water, a head may form at this end. If the animal is covered only by an exceedingly thin layer of sand, a polyp will still be formed which makes its appearance between the grains of sand, much as the stem of a germinating seed may grow through a thin layer of earth.

One of the poles of a piece of a stem was pushed between two slides laid upon each other and held together by thin rubber bands. Needles were placed between the two slides, and one end of the stem of the Tubularian was laid in the wedge-shaped space thus formed. In this way the end was subjected to slight pressure. No polyp was formed at the end subjected to this slight pressure, no matter how long I waited; while at the other end, which was not pressed upon and was surrounded by water, a polyp was formed in the usual time. When the piece was removed from between the slides, a new polyp frequently developed at the end that had been subjected to the pressure. That light is not necessary to the formation of a polyp was proved by the fact that pieces of Tubularian stem will grow new polyps in a darkened vessel. The experiments described were made in wellaërated aquaria.

4. When the polyps and the roots are cut off from long stems of Tubularia mesembryanthemum, it is found that the new polyps are always formed one, two, or three days earlier at the oral than at the aboral end. I believe that the cause of this phenomenon, which may be considered as an intimation of "polarity," lies in the fact that when long pieces are 
cut from the stem, the lumen at the cut oral end is usually wider than that at the aboral end; for when I cut from the middle of the stem shorter pieces, which showed no difference in the diameter of the lumina, a polyp often formed earlier at the aboral end than at the oral.

5. The size of the newly formed polyp also depends to a certain extent upon the diameter of the stem at the cut end. When the diameter was very small, the polyp was also very small; when the diameter was large, the polyp was also larger.

6. It might still be imagined that, besides the mechanical factors thus far considered, a physiological factor might also play a rôle. It might be thought that the substance of which the polyp is formed is present in a larger amount at the oral than at the aboral pole. To test this point I chose a large number of very long Tubularian stems that had been cut off close to the roots, and at the cut ends of which polyps had been grown. I bisected these stems transversely, and kept the oral and aboral halves in separate beakers. If the substance required for the formation of the polyps were unequally distributed in the stem, then the one series of fragments should have formed polyps sooner than the other series. This was never the case; but — as was again noted - every fragment formed a polyp sooner at its oral than at its aboral end, even though the difference in time often amounted to only one half-day or less.

7. While I have always succeeded - with suitable material, and with the experiment under the proper external conditions - in making a head grow at the aboral end of the stem, I have thus far not yet succeeded in making a root grow at the oral end of a stem. When I cut off the stems close to the substratum to which the roots were attached and brought the aboral ends in contact with the walls of the aquarium, the end, when it grew at all, attached itself to the 
solid body and became a root; however, when contact with the wall of the aquarium was broken so that water surrounded the root on all sides, a polyp was formed also at the end of the root. In my further experiments I shall try to find conditions under which the animal will form roots at both poles with just as great certainty as it now forms heads. From the experiments thus far discussed, I can only conclude that the formation of polyps in Tubularia mesembryanthemum can be brought about much more easily than the formation of roots.

III. THE LIFE-PHENOMENA OF THE ORAL POLE OF TUBULARIA MESEMBRYANTHEMUM

Doubt might arise as to whether the two heads of a bioral Tubularian manifest the same life-phenomena; as to whether the two morphologically equal poles are also identical physiologically. I shall show that this is, indeed, the case, and in doing so shall dwell a little more upon the differences in the irritability of stem and root.

1. The stem and root of Tubularia mesembryanthemum have an entirely different contact-irritability. If the root is brought in contact with a solid body, it attaches itself to it, and in its further growth remains closely attached to the surface of the solid. If an attempt is made to lift the stem from the solid body, it tears off close to the root, the latter remaining attached to the base upon which it grew. The polyp has exactly the opposite irritability. When the polyp comes in contact with a solid body-for example, when the stem lies horizontally upon the bottom of the aquarium - it soon grows away from it. The growing region of the stem (which is situated close behind the polyp) becomes convex against the solid substratum.

This (stereotropic) bending occurs only in the growing part of the stem, and persists when growth has ceased, just 
as do geotropic or heliotropic curvatures in many growing plants. To bring about this stereotropic curvature it is necessary that the polyp itself should come in contact with the solid body. If any part of the stem alone comes in contact with the solid, no bending occurs, even though the growing part of the stem, close to the polyp, touches the solid. The contact-irritability of the polyp is opposite in kind to that of the stem; the stem is positively, the polyp is negatively, stereotropic.

The negative stereotropism of the polyp may be clearly demonstrated in the following simple manner: Beheaded Tubularians were fixed in a beaker half-filled with sand in such a way that one end was fixed in the sand, while the other end just touched the side of the vessel. As soon as the new polyps were formed and the Hydroids began to grow in length, the tips of all the stems bent away from the glass sides of the vessel. The direction of the rays of light had no effect upon this process.

In all these experiments the polyps formed at the aboral end behaved exactly like those formed at the oral end.

2. I have not succeeded in bringing about either heliotropic or geotropic curvatures in Tubularia mesembryanthemum. When I fastened the stem in the middle, and when both ends were surrounded by sea-water on all sides, the stem of the bioral Tubularian continued to grow in the direction of the old piece; it mattered not whether it lay in a vertical or in a horizontal position, or in which direction the light struck it. This is a remarkable fact, for, in looking at a colony of Tubularians, one might easily be led to think that they possess heliotropic or geotropic irritability, as the stems of such a colony upon the surface of a solid are all arranged in the same way. Yet the similarity in the orientation might be determined in the main by their contact-irritability. The oral ends of the young stems 
grow almost perpendicularly away from their substratum. If, in addition, the separate stems stand very close together, as is usually the case, the contact of the polyps with each other influences their orientation. This has the same effect as would be brought about by causing each separate polyp to grow in a narrow hollow cylinder. The individual stems must thus not only grow away from the surfaces to which they are attached, but they must grow away from it in approximately straight lines.

3. Dalyell has observed in Tubularia indivisa-a form very similar to Tubularia mesembryanthemum-that the polyps drop off after they have existed a certain length of time, and that after a longer or shorter period new polyps are formed in their places. As soon as a new polyp has been formed, the stem begins to grow in length immediately under it. The growth continues as long as the polyp exists; as soon as it drops off, growth ceases. ${ }^{1}$

I observed the same condition of growth in Tubularia mesembryanthemum. The longitudinal growth of the stem was continued to a region just beneath the polyp, and it continued as long as the polyp existed; when the latter dropped off, growth ceased; when a new polyp was formed, the stem again grew in length. In the bioral polyps an increase in length occurred simultaneously at both ends of the stem, so that these stems reached a much greater length in a shorter time than any of the normal specimens that were ever brought to me by the collectors of the Zoölogical Station in Naples. That the stem grows in length close behind the polyps at both ends of the bioral animal is clearly shown by the fact that the newly formed part is thin and transparent, and thus can be readily distinguished from the older opaque portions of the stem. Therefore in its growth also the aboral pole of Tubularia behaves like the oral.

1 Rare and Remarkable Animals of Scotland (London, 1847). 
4. I did not succeed in observing the polyps in the process of taking up food. Yet I have noticed in both the oral and the aboral polyps the same sudden closure of the tentacles which occurs in Actinians when they seize their food and swallow it. I shall show later in Actinians that heads which have been newly formed in abnormal places behave like normal heads in the matter of taking up food.

IV. THE DISCREPANCY BETWEEN ALLMAN'S THEORY OF POLARITY AND THE BEHAVIOR OF TUBULARIA MESEMBRYANTHEMUM

1. I mentioned in the introduction that Allman based his theory of polarity on observations made upon Tubularia. The discrepancy between Allman's ideas and my observations compels me to enter into a more detailed discussion of his theory.

The passage in Allman's treatise which is of interest to us is the following:

There is thus manifested in the formative force of the Tubularia stem a well-marked polarity, which is rendered very apparent if a segment be cut out from the center of the stem. In this case, no matter in what position the segment may be, that end of it which was directed downward or proximally, while it formed a part of the unmutilated hydroid, will never develop a polypite, but will extend itself as a simple prolongation of the coenosarc; while the upper or distal end, instead of becoming simply elongated, will shape itself into a true polypite; and all this is true, though of course not the least difference in structure or form can be detected between the two extremities at the time of section. ${ }^{1}$

Allman adds in a note that the observations of Dalyell, who made numerous regeneration experiments upon Tubularia indivisa, are in perfect accord with his own. By reading Dalyell's paper one, indeed, finds the same idea expressed as by Allman, although the term "polarity" is not used. It might be thought that Tubularia indivisa, upon

1 Loc. cit., pp. 392 ff. 
which Allman and Dalyell experimented, behaves typically differently from Tubularia mesembryanthemum, upon which I made my experiments. A certain difference seems, indeed, to exist. Dalyell states that the stem of the Tubularia indivisa bends upward, when laid horizontally; this I have not observed in Tubularia mesembryanthemum.

Yet I do not believe that the conditions determining the form of Tubularia indivisa differ in principle from those in Tubularia mesembryanthemum. For Dalyell notes that he once observed the growth of polyps from both ends of a piece cut from the middle of a Tubularia indivisa. "It may be conjectured that the summit of both had originally constituted a single embryo, which by partition developed into two, becoming progressively symmetrical in maturity."

To explain the formation of a head at both ends of the stem in the single case just described, Dalyell therefore assumes that the new head divided in the course of its development. Such a division would, in consequence, always have to occur in the case of Tubularia mesembryanthemum, which without exception forms a head at both ends, if both ends are surrounded by water and have a sufficiently great diameter, and a dividing embryo would therefore have to exist in every piece of the stem of Tubularia mesembryanthemum. Even if one were willing to consider this hypothesis, it yet could not be made to harmonize with Allman's theory of polarity; for, according to this theory, both embryos would necessarily have to develop always at the same end, namely, at the oral one; yet I have never found two heads to develop here side by side.

2. I might mention that it is possible apparently to obtain such results in Tubularia mesembryanthemum as Allman describes, if the stems used in the experiments are cut off close to the root, and if care is taken, in choosing the

1 Loc. cit., p. 35. 
specimens, to select only those which are very thin at the base. Such a selection might easily be made accidentally in an experiment. In this case one might notice that polyps arise only at the oral end, especially if the experiments are not continued for a very long time. Just as Allman regards such a behavior as the expression of polarity in the animal body, some botanists speak in analogous cases of "morphological forces." I believe that the "morphological force" which decides that a polyp forms first at the oral end of a Tubularian segment is essentially nothing more than that the diameter of the tube is very small at the aboral end of the stem. Yet I prefer not to enter into a discussion of such hypothetical things in this paper.

\section{HETEROMORPHOSIS IN AGLAOPHENIA PLUMA}

While in Tubularia we dealt with but a single stem which under ordinary conditions ends in a root at one end and in a polyp at the other, we have to deal in what follows with colonies of animals. The place of the head is here taken by a more or less ramified stem possessing many polyps. At the other end is formed a root (as in Tubularia). We shall confine ourselves to experiments upon the stems. We shall call the end directed toward the root the aboral or basal end of the animal; the other, free end, the oral or apical end. I wished to determine whether it was possible to make a new tip grow in place of the root at the basal end of the stem, or vice versa, and how we might accomplish this.

1. Aglaophenia pluma (see Figs. 17-19) consists of a main stem from which lateral branches are given off on both sides. These side branches carry polyps upon their upper surfaces; they are slightly convex toward the tip of the main stem and arise from it at an acute angle, which opens toward the tip of the main stem. The side branches are the shorter 
the nearer the tip they are. These points enable one to distinguish between the basal end (originally directed toward the root) and the apical end (originally directed toward the tip) of a stem from which the tip and root have been cut.

2. I cut off some stems of Aglaophenia pluma close to the

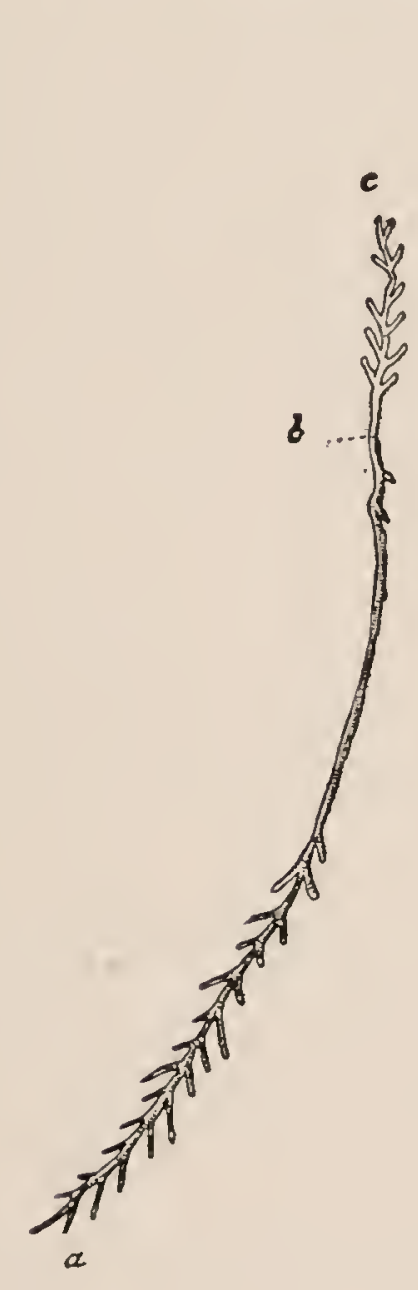

FIG. 17

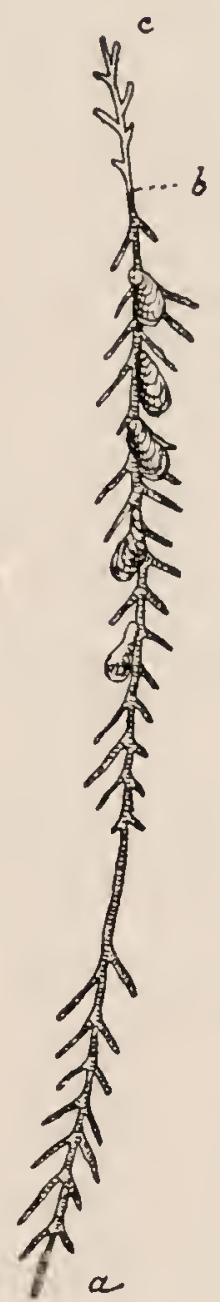

FIG. 18

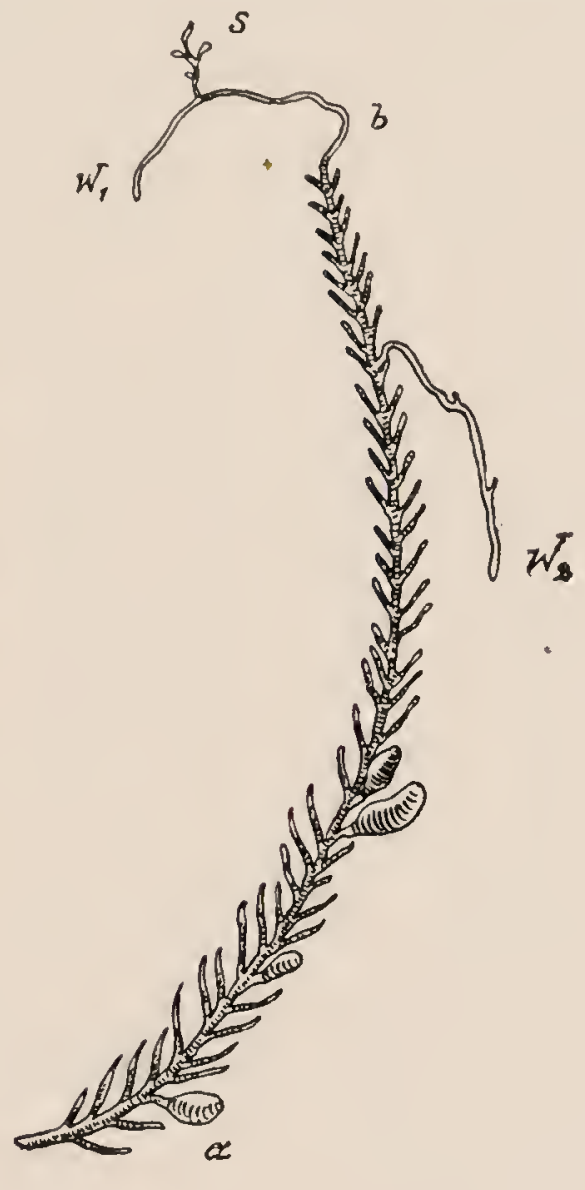

FIG. 19

root, and fixed them vertically, but with their tips downward, into the sand. The tips were planted just deeply enough to keep the animals in a vertical position. The remaining part of the stem was surrounded by water. In a number of these animals new tips, which continued to grow upward, were formed at the basal ends (Figs. 18, 19). At first the old main stem grew in length by growing vertically upward. From this there then arose the lateral branches. The new polyps which were formed grew only upon the 
upper surfaces of the lateral branches, and were therefore directed, not toward the old, but toward the new tip of the animal. Furthermore, the acute angle at which the new lateral branches arose from the main stem opened toward the zenith; the convexity of the new branches was also directed toward the zenith. In this way animals were therefore formed which ended in a tip at both ends - animals that were biapical; just as though one were to grow a new top upon a tree in place of the roots, without, however, allowing the old apex to go to pieces. In the specimens illustrated in this paper the tips are still relatively small. My stay at Naples was too short to allow me to wait for them to reach maturity.

3. When stems of Aglaophenia which had been cut off close to the roots, the tips of which, however, were left intact, were suspended vertically and in an upright position in water, a new root was invariably formed at the basal end, and never a new tip.

It therefore seems that the position of the stem of Aglaophenia determines to a certain extent whether a heteromorphosis, or only a regeneration, of the lost part occurs at the basal cut end.

4. This fact is further supported by the following observation: When stems of Aglaophenia from which the tips and roots had been cut were suspended vertically in the aquarium so that both cut ends were surrounded by water, a root was always formed upon the end directed downward, it mattered not whether it was the basal or apical end.

In many cases branches were formed at the end directed upward, yet in other cases a root was formed here also. A root was formed most frequently upon the ends directed upward when the basal end of the stem was pointed in that direction; branches were formed most frequently when the apical end of the stem was directed upward.

It is therefore possible to create bibasal Aglaopheniae, 
and, according to the experiments thus far made, the method by which this is accomplished consists in cutting off the tips and roots of the stems, and suspending the stems vertically in the aquarium with their bases pointing upward.

5. When such segments from which the tips and the roots have been cut off are laid horizontally, and in such a way that they are surrounded by water on all sides, roots grow only from the aboral ends of the fragments, while, as a rule, new tips grow from the oral ends. Exceptionally, however, roots grow from the oral ends also.

6. Bearing in mind the fact that roots may arise from either cut end and under all conditions, we may say that biapical animals may be produced by leaving the tips intact and cutting off the stems close to the root. If such stems are suspended vertically, with their tips downward, new tips may arise at the aboral end. If bibasal animals are desired, stems deprived of their tips are cut off close to the root, and are suspended vertically in the aquarium, with their tips downward. In all my experiments thus far performed only roots have been formed at the cut ends directed downward, while tips or roots have been formed at the cut ends directed upuard. Besides the influence which the position of the stem has upon the formation of organs, another at present unknown, and therefore uncontrollable, factor exists which renders possible the growth of a root at the cut end which is directed upward. Yet I believe it possible that purely external conditions (which were satisfied in the aquarium, and which possibly some day may be brought under control) determine this strong tendency toward the formation of roots.

It still remains to be investigated whether gravity or light or both circumstances have an influence upon the formation of the organs in this case. In all my experiments performed thus far in the dark room, no regeneration whatsoever of the 
lost organs occurred-a fact probably not entirely due to lack of light.

7. The roots were characterized by a distinct kind of contact-irritability and by a tendency to bend downward, which I shall now discuss.

When a root was formed at the cut end of a vertical stem, it at first grew horizontally for a short distance-when it did not come in contact with solid bodies - and then downward (Fig. 19, $w_{1}$ ). In stems lying horizontally the root grew directly downward. In animals thus operated upon, adventitious roots were also often formed at the middle of the stem. I have never found these adventitious roots upon the uninjured animals taken from the ocean. They grew directly downward toward the earth (Fig. 19, $w_{2}$ ). The phenomenon seemed strangest of all when such adventitious roots arose from a stem fixed in the sand in an inverted position (with the tip down); in this case the root grew toward the apical end of the animal. At times these downward-growing roots showed torsions such as are found in winding plants.

8. The newly formed main stems behave in a way opposite to that of the roots; they grow vertically upward. This contrast between the root and main stem is shown most beautifully when new stems with polyps arise from the newly formed root itself. In Fig. 19 is shown a branch which, after having been deprived of its tip, was fixed vertically in the sand with its tip directed upward. In place of the tip a new root $w_{1}$ grew from the main stem, at first horizontally and then downward. A young branch $s$ arises from the root $w_{1}$ and grows vertically upward.

In another stem all the lateral branches had gone to pieces; it had been suspended vertically. I believed that the animal had died, when from the middle of the stem branches began to arise, which proved to be both roots and polyps; the roots sprang from the lower portions of the stem, 
the new stem from the upper portions. The new stems grew upward, the roots downward. I have seen such new stems arise, not only from the main stem and the main roots, but also from the adrentitious roots. Here also the new stems always grew upward. Finally, I have seen new stems, which also grew upward, arise from stems lying horizontally. When, however, I cut off the tip from stems lying horizontally, and regeneration occurred without heteromorphosis or deformity of any kind, the new tip showed, so far as my present experience goes, no tendency to bend upward.

9. All newly formed stems arose from the upper surface of the stem or root (see Fig. 19, s), it mattered not whether they grew upon the main stem or upon the accessory roots. The accessory roots sprang from the lower surface of the stems when these lay horizontally. Whether all these phenomena are determined solely by gravity I shall attempt to decide by further experiment.

10. That form of contact-irritability which I have called stereotropism plays an important rôle in the growth of the root of Aglaophenia. When the roots come in contact with a solid body, they attach themselves to it (by means of a secretion?) and grow along its surface. This attachment is a phenomenon of irritability which is called forth by contact with the solid body itself; for when the root is brought in contact with a solid body, it does not immediately stick to it, but only after contact has lasted for some time (often as long as twenty-four hours). Only the growing part (tip) of the root is able to fasten itself to the surface of a slide. The root adheres so firmly to the solid body that it is impossible to separate the two by traction; the root tears before it can be pulled from the solid body. I have not as yet observed the branches of Aglaophenia bend away from a solid body. Yet I have proved with certainty that growing branches carrying polyps never attach themselves to a solid body with 
which they are brought in contact, no matter how long one may wait; while, on the other hand, this reaction is always obtained with certainty in growing roots. I have also assured myself of the fact that when adventitious roots are brought in contact with the wall of a beaker, they immediately attach themselves to it, and grow on its surface.

11. As soon as a root comes in contact with a solid body, it grows rapidly in length, and in a few days exceeds the length of other newly formed and equally long roots, which are prevented from contact with solid bodies. ${ }^{1}$

The attachment of the root to a solid body influences form in a second direction. While we observed above that new stems grow from the upper surface of a root when it is surrounded on all sides by water, we find, when the root is attached to a solid substratum, that the stems arise from that surface of the root which lies opposite the solid body. In their further development these stems also grow only straight upward, though not entirely vertically.

12. I have observed in the roots of Aglaophenia a phenomenon relative to growth which has thus far been known only in plants.

The longitudinal growth of the root is confined to a narrow region situated near the tip (while no longitudinal growth occurs in the remaining portions of the root). This could be shown in the following way: I permitted the roots of Aglaophenia to attach themselves to a slide and grow upon it. A slight bulging out soon occurred just behind the tips - the beginning of a new stem, which on the next day reached a length of $\frac{1}{2} \mathrm{~mm}$. and soon thereafter bore polyps. I marked the position of the beginning stem on the glass, by etching a line into the glass. The position of this new

1 DALYELL observed in Sertularia halecina that new growths occur which adhere to other solid bodies and thereby become abnormally long. "These coming in contact with a solid surface have a tendency to adhere and to extend in irregular prolongations surpassing the natural increment," Rare and Remarkable Animals of Scotland (London, 1847), p. 165. 
stem upon the glass remained permanently the same, though the tip itself moved forward at the rate of about $1 \mathrm{~mm}$. each day. The longitudinal growth must, therefore, have occurred in the narrow zone lying in front of the new stem.

13. These experiments were made in April when the

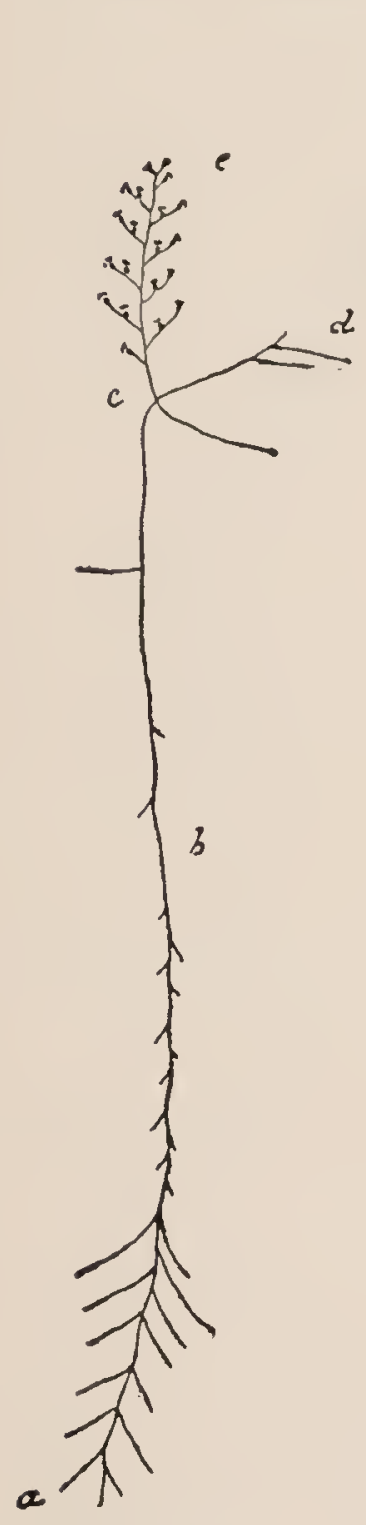

FIG. 20a

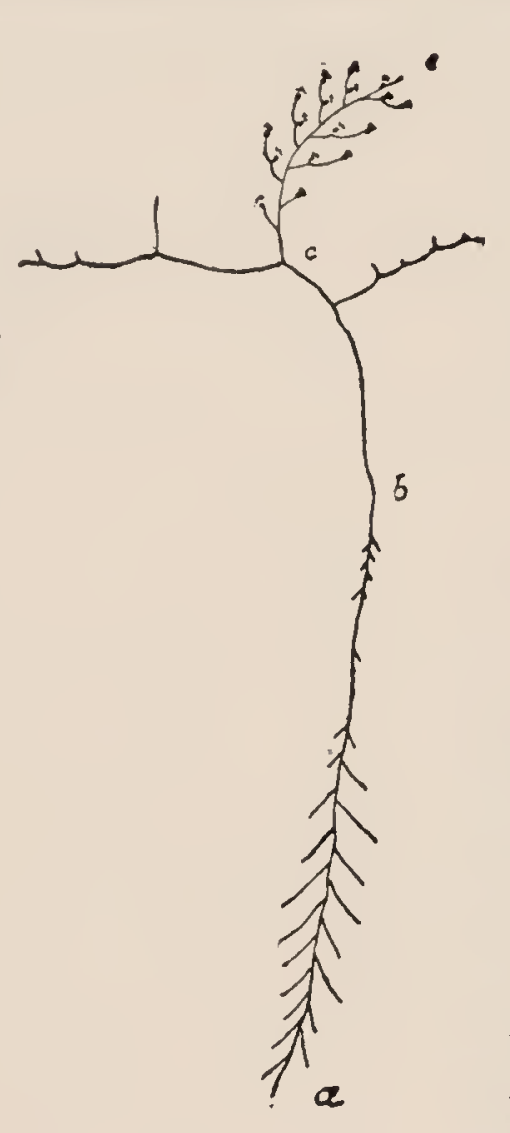

Aglaophenix were sexually mature. One day I observed a number of small (about $\frac{1}{2} \mathrm{~mm}$. long), whitish, cone-shaped larvæ that moved over the bottom of the aquarium toward the window, and remained there. The next morning, however, they had all disappeared, so that I can only suspect that these organisms, which in the moment of observation were positively heliotropic, may have been larvæ of Aglaophenia.

\section{HETEROMORPHOSIS IN PLUMULARIA PINNATA}

1. I have made a series of FIG. $20 b$ experiments, similar to those made upon Aglaophenia, upon Plumularia pinnata, which in form closely resembles Aglaophenia pluma. I wish to describe one of these experiments here.

A series of stalks were cut off close to the root and fixed vertically in the sand, so that the apical ends were covered by it. In individual instances, but only very rarely, a new tip was immediately formed at the aboral end, so that I ohtained biapical animals similar to those of Aglaophenia 
pluma; the new apex, however, was not so regularly formed. Occasionally a lateral branch was missing upon the side of the main stem. In the majority of the experiments, however, such figures as are shown in Figs. $20 a$ and $20 b$ were formed. A new piece $b c$ at first grew vertically upward from the main stem $a b$ until the new stem $c e$ was formed, which usually grew vertically upward; the polyps of this new stem $c e$ were all located upon the upper surfaces of the lateral branches, so that the new stem $c e$ was oriented symmetrically to the old stem $a b$ with respect to a horizontal axis. After this the main stem began to grow horizontally and finally downward.

2: The newly formed parts $b c$ arising from the prolongation of the main stem, and growing at first horizontally and then downward, all possessed the contact-irritability of roots, namely, positive stereotropism. When brought in contact with solid bodies, they attached themselves to their surfaces and behaved like the roots of Aglaophenia pluma. Only the growing parts of the roots were able to attach themselves in this way. Here also the influence of contact stimuli in determining the point of origin of branches again showed itself. While the branches arose, almost without exception, from the upper surface of the root, when it was surrounded by water, they were formed upon the side opposite the solid substratum upon which the roots grew, in roots which were attached to the surface of a solid body.

The experiments were made in an aquarium which was far removed from a window, and into which only very weak light fell almost horizontally. In spite of this, the branches grew vertically. This seems to indicate that light has no influence in this case in determining the place where new organs are formed.

The protoplasm retracted from that portion of the branch which was buried in the sand. 


\section{HETEROMORPHOSIS IN EUDENDRIUM (RACEMOSUM?)}

Eudendrium (racemosum?) (Figs. 21a and 21b) consists of a main stem which terminates in a polyp at its upper end and in a root at its lower end. The root adheres to solid bodies. Stout lateral branches arise from the stem and

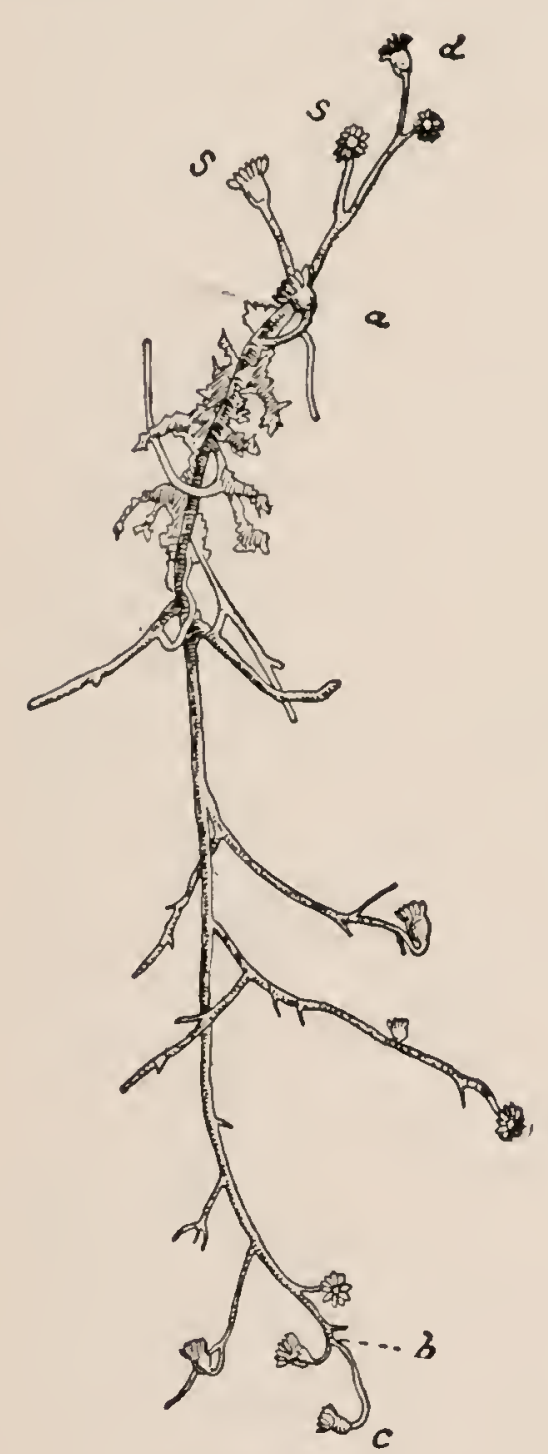

FIG. $21 a$

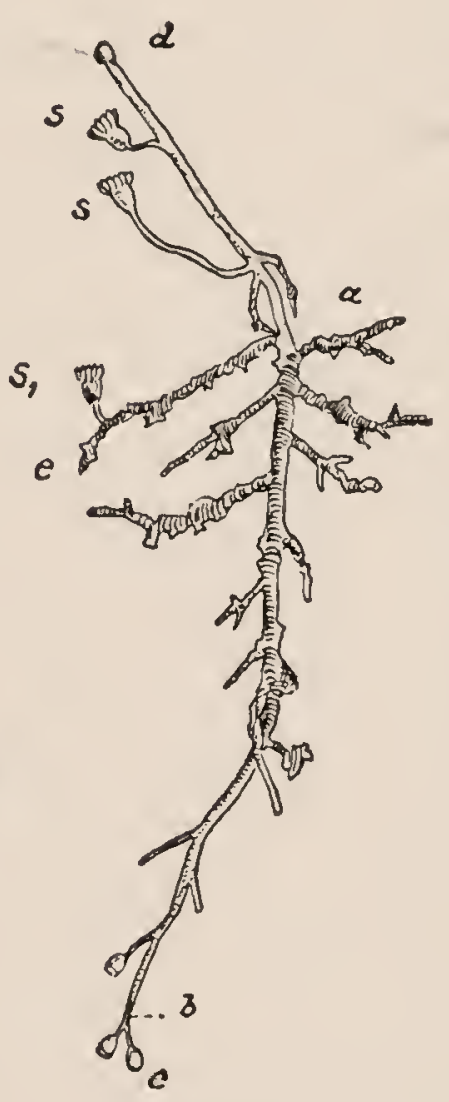

FIG. $21 b$ grow upward. They also carry polyps at their tips. New branches may again arise from these, all of which are directed toward the tip of the main stem. I cut off the tips and roots from stems of Eudendrium and suspended them in part with the tip, in part with the base directed downward in the aquarium. Both ends were surrounded by water. The stem began to grow from the two extremities, and polyps were formed at both ends (Figs. 21a and 21b). All Eudendria became biapical (just as does Tubularia mesembryanthemum under similar conditions); with this difference, however, that in addition to the new tip, roots were at times formed, at one of the cut ends, which was never the case in Tubularia.

To maintain the pieces of Eudendrium stems in a vertical position in the aquarium, I pushed them through lead plates in which fine holes had been punched. The plates rested 
upon beakers. While the upper end of the animal was, therefore, in the aquarium, in which the sea water was continually renewed, the lower ends of the stems dipped into the beakers in which the circulation of the water was much less perfect than in the rest of the aquarium. Striking differences existed between the new growth which occurred at the lower end and that at the opposite end. The lower end in the poorly aërated water formed a new polyp upon the main stem, but its growth was slow, and the formation of new lateral branches occurred either not at all or only slightly as compared with the corresponding processes at the other end. (Possibly light and gravity may also have played a rôle in bringing about this result.) In what follows we shall consider only the new growths which occurred at the upper end of the vertically standing stem.

2. When the basal end of the stem was directed upward, and new side branches were formed, they were directed, not toward the old tip, but toward the new tip. In Figs. $21 a$ and $21 b a b$ is the old stem, $b c$ the regenerated tip, and $a d$ the heteromorphic tip at the aboral end. The newly formed branches $s$ are all directed toward the heteromorphic tip $d$.

In a larger number of cases new stems were formed also upon the old lateral branches after the stem had been turned upside down. Some of these did not grow downward toward the old tip, but in the opposite direction, upward, toward the new tip. Fig. $21 b$ illustrates such an instance. After the whole stem had been suspended in an inverted positjon in the aquarium, a new branch $s_{1}$ was formed upon the lateral branch $e$, and grew upward, toward the new tip. It had, moreover, been formed upon the upper surface of the branch $e$.

In the arrangement of new organs in Eudendrium we do not, therefore, deal with a "polarity" which is determined solely by internal structural relations, but with the effects of 
stimuli in which not only the internal structural conditions, but these and the external stimuli together, determine the result. The external stimuli with which we deal here seem to be light and possibly gravity.

3. The effect of light upon the formation of new polyps in Eudendrium is shown in an unmistakable way. When one compares the number of new polyps and branches formed upon the window side of the stem with those formed upon the room side, one finds that the number upon the window side is very much the larger.

The branches are, moreover, positively heliotropic. I cut off a Eudendrium at its base, close above the root; a new polyp was formed upon the tip of the stump that remained. The stem then began to grow rapidly. The growing, apical portion of the stem bent toward the window side of the aquarium. That part of the stem which was not growing actively showed no heliotropic curvature.

4. I have made a single observation which seems to indicate that currents in the water, if they are continued for some time and always in the same direction, can produce curvatures in a growing Eudendrium stem. The anal opening of a large Ascidian was situated near a growing Eudendrium stem, so that the stream of water ejected by the Ascidian struck the Eudendrium. The growing part of the Eudendrium which was struck by the current of water bent so as to have its concave side directed toward the source of the current. The other stems of the same culture which had been subjected to otherwise similar treatment had all bent toward the source of light. This observation also shows that the rheotropism of these Eudendrium stems - if we are, indeed, dealing with this phenomenonis able to overcome and to veil their heliotropism.

5 . In a few cases in which contact had been especially close, roots were formed in the middle of the stem, where it 
had been in contact with the lead plate. These roots attached themselves to the lead plates and spread over their surfaces. I have not yet succeeded in producing bibasal Eudendria.

VIII. HETEROMORPHOSIS IN SERTULARIA (POLYZONIAS ?)

Heteromorphosis can be produced in Sertularia; but just as the heteromorphoses in no two of the animals thus far

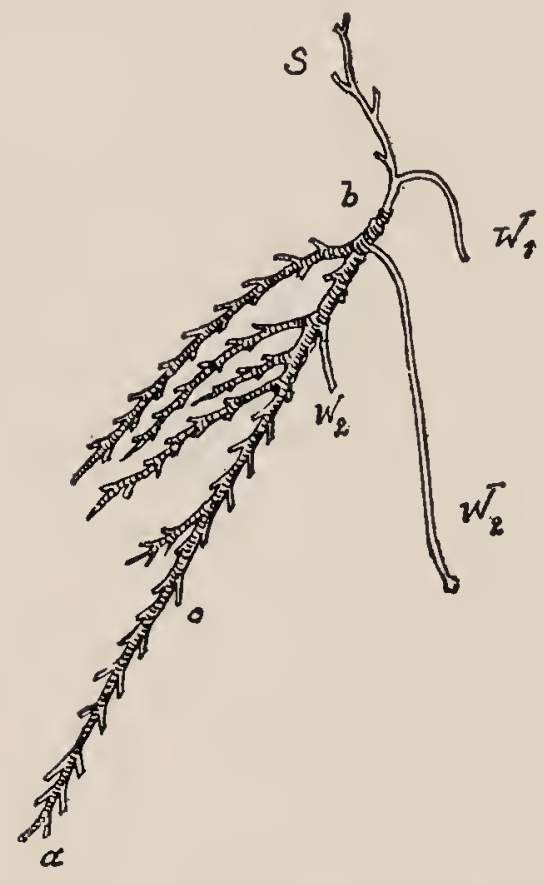

FIG. $22 a$

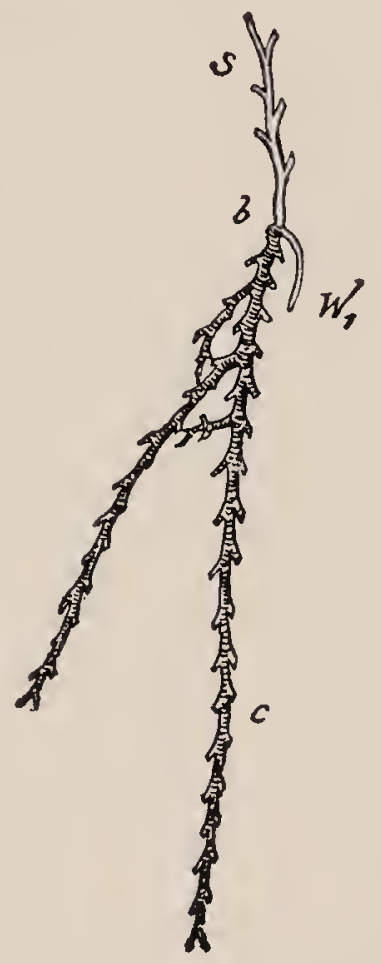

FIG. $22 b$

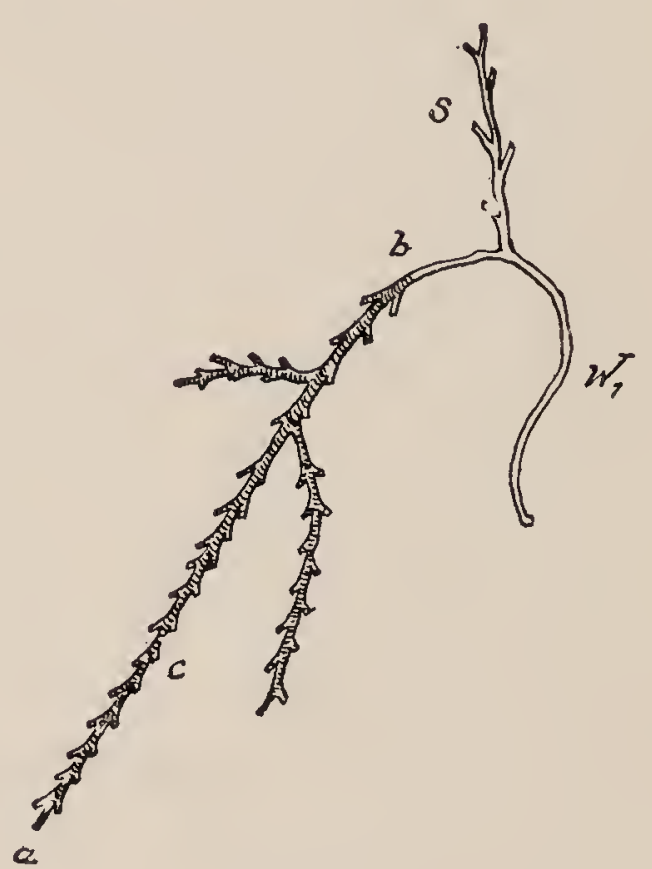

FIG. $22 c$

considered are exactly similar in every detail, heteromorphosis in Sertularia has its specific characteristics also. I cut off stems of Sertularia close to the root and fixed them in an inverted position in the sand. Both roots and stems (Figs. 22a and 22b) grew from the cut basal end. But while the stems grew upward (and in the direction of the rays of light), the roots grew downward (and away from the source of light). ${ }^{1} \quad$ As shown in my earlier observations, the roots of Sertularia are negatively, the branches positively, heliotropic. Biapical stems can be easily produced in the manner described. Not uncommonly the condition represented in Fig. $22 c$ is found, in which a negatively helio-

1 See paper ii, p. 89. 
tropic root arises from the main stem, and from this (upon the side directed toward the light) spring new positively heliotropic stems. This arrangement corresponds with that so often found in Plumularia pinnata.

The roots are positively stereotropic as in the other Hydrozoa. When brought in contact with a solid body they attach themselves to its surface. As soon as the roots have attached themselves, the position of the new stems forming upon the roots is determined by the contact stimulus; the new stems arise from those points on the surface of the root which are diametrically opposite the substratum to which the root is attached.

The fact that roots and stems may arise simultaneously and side by side from the basal end of an organ has also been observed in certain organs of plants; e.g., in fragments of leaves which form both roots and stems at their bases. The protoplasm retracted from that piece of the Sertularia stem which was covered by sand.

2. When the tips were cut off of stems which were fixed in the sand in a vertical and upright position (with the tip upward), simple regeneration of the tip followed in the great majority of cases. Only once or twice did I see a root arise from the tip of a vertical and upright stem.

3. In inverted stems occasionally new stems arose from the middle of the old stem upon the side directed toward the source of light. These grew in a direction determiner by their positive heliotropism; when the light came from above, they grew upward toward the basal cut end.

Roots which were formed in the middle of inverted stems (Fig. 22a) grew downward and toward the room side of the aquarium, when the light fell upon them from above.

4. Driesch has observed a phenomenon of growth in specimens of Sertularella polyzonias which were cultivated under "unfavorable" conditions that I have never found in 


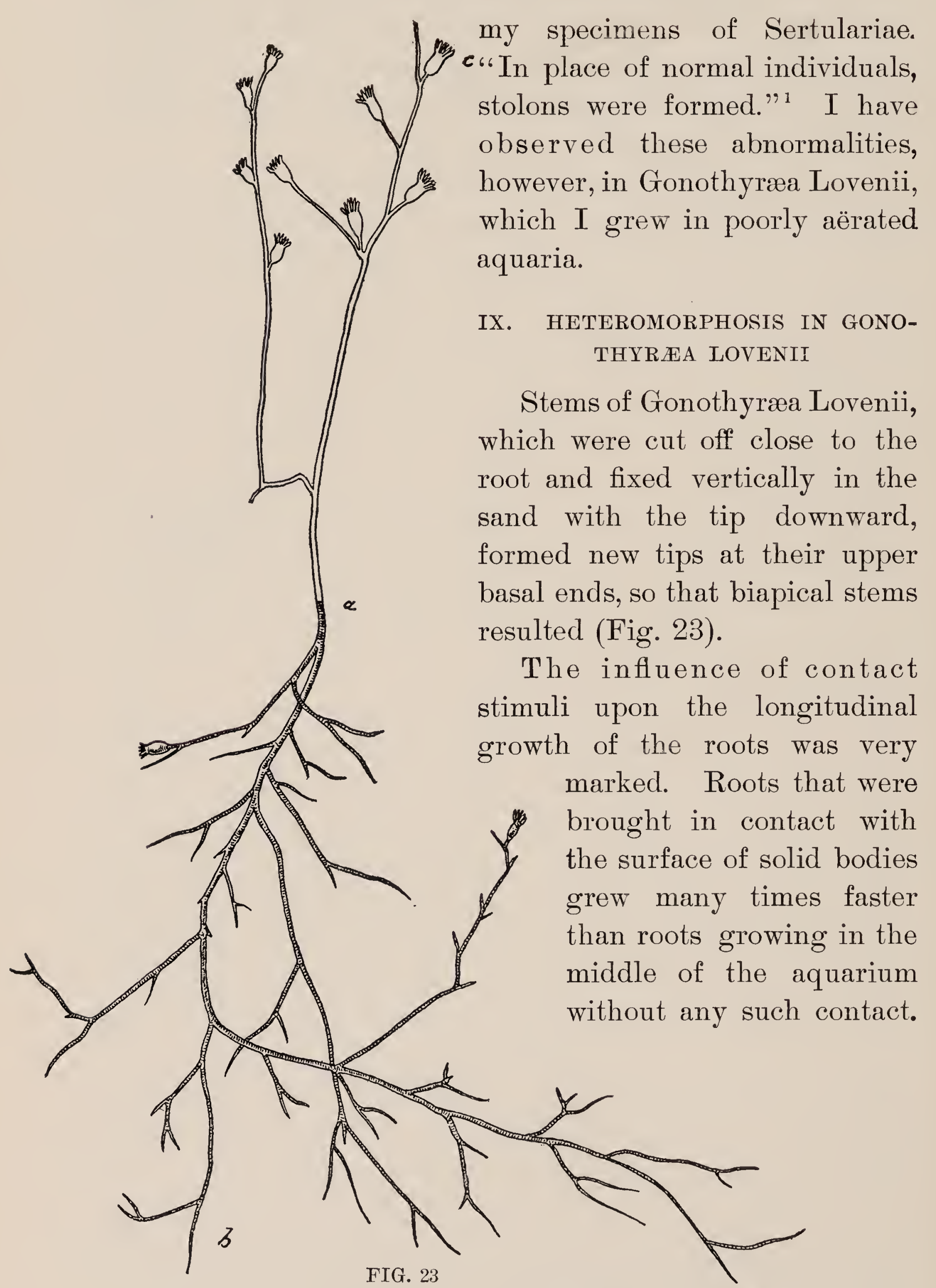

1 H. DRIEsCH, "Heliotropismus bei Hydroidpolypen," Zoologische Jahrbücher, herausgegeben von SPENGEL, Vol. V, p. 150. 
When the roots were brought in touch with the surface of the water, the latter acted upon the root as a solid body. The root began to grow rapidly in length, attaching itself to the surface of the water (as if it were the surface of a solid body). Whenever a root adhered to a solid body new stems arose from that surface of the root which lay diametrically opposite to the solid body. Usually these branches then grew

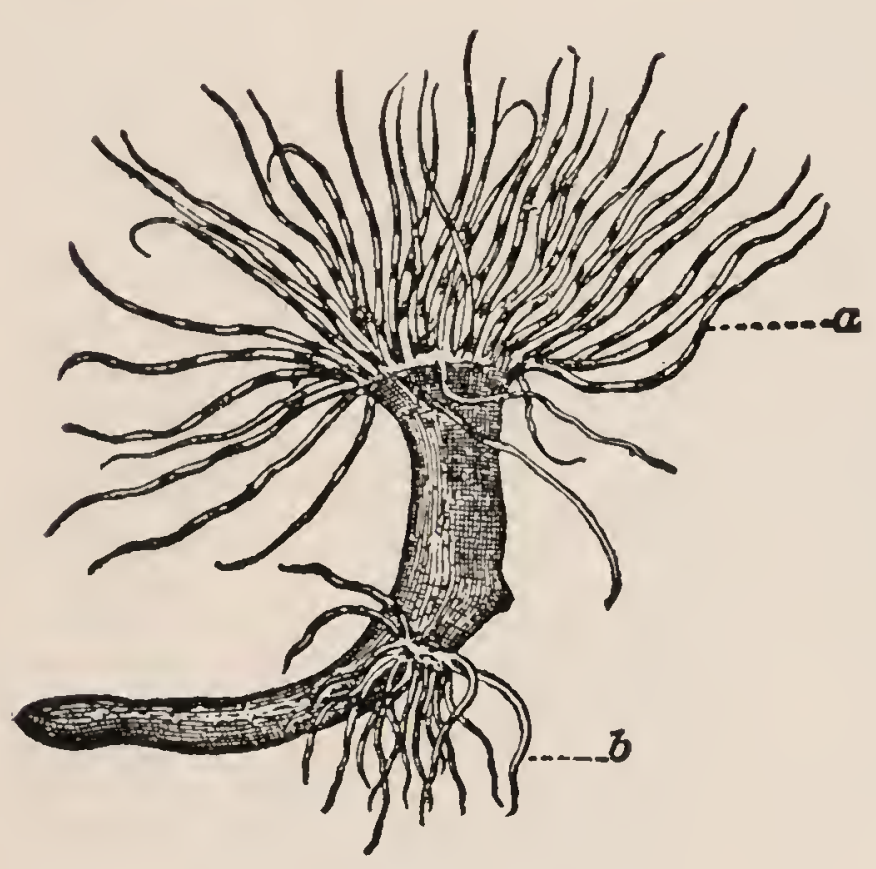

FIG, 24

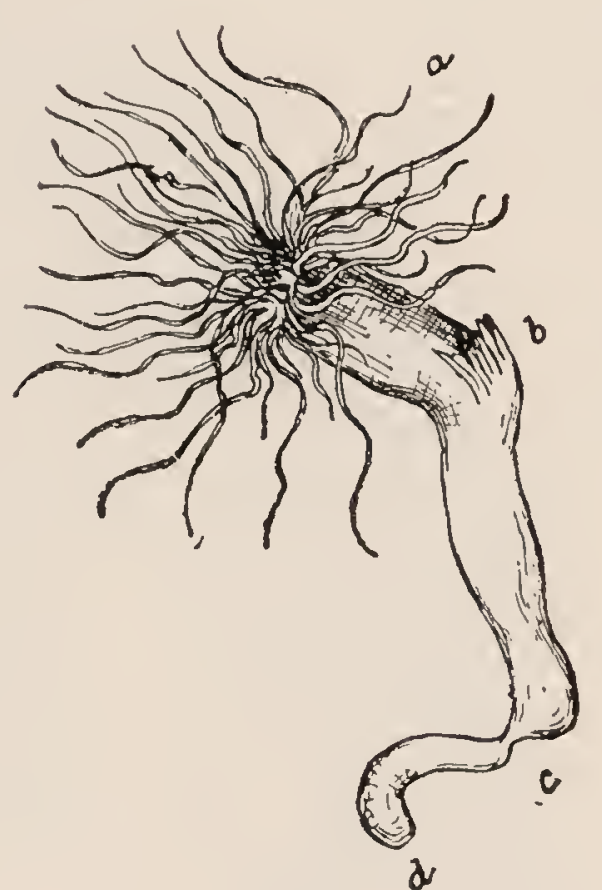

FIG. 25

perpendicularly away from the surface of the solid body. When new stems arose from roots growing along the surface of the water, the stems grew vertically downward.

\section{ON THE FORMATION OF TENTACLES IN CERIANTHUS MEMBRANACEUS}

1. I shall now discuss some experiments upon animals which seem to behave in accordance with Allman's theory of polarity, inasmuch as in these I did not succeed in producing a head in the place of an aboral pole. The experiments led however to the production of several heads lying one above the other in one and the same animal (see Figs. 24, 25). The irritability of the new heads could easily be compared with that of the old. In these experiments, more- 
over, I had the opportunity of studying new relations between irritability and body form, and in addition one of the fundamental conditions which underlie growth. These latter observations point to a greater similarity between the general life-phenomena of animals and plants than has thus far been known.

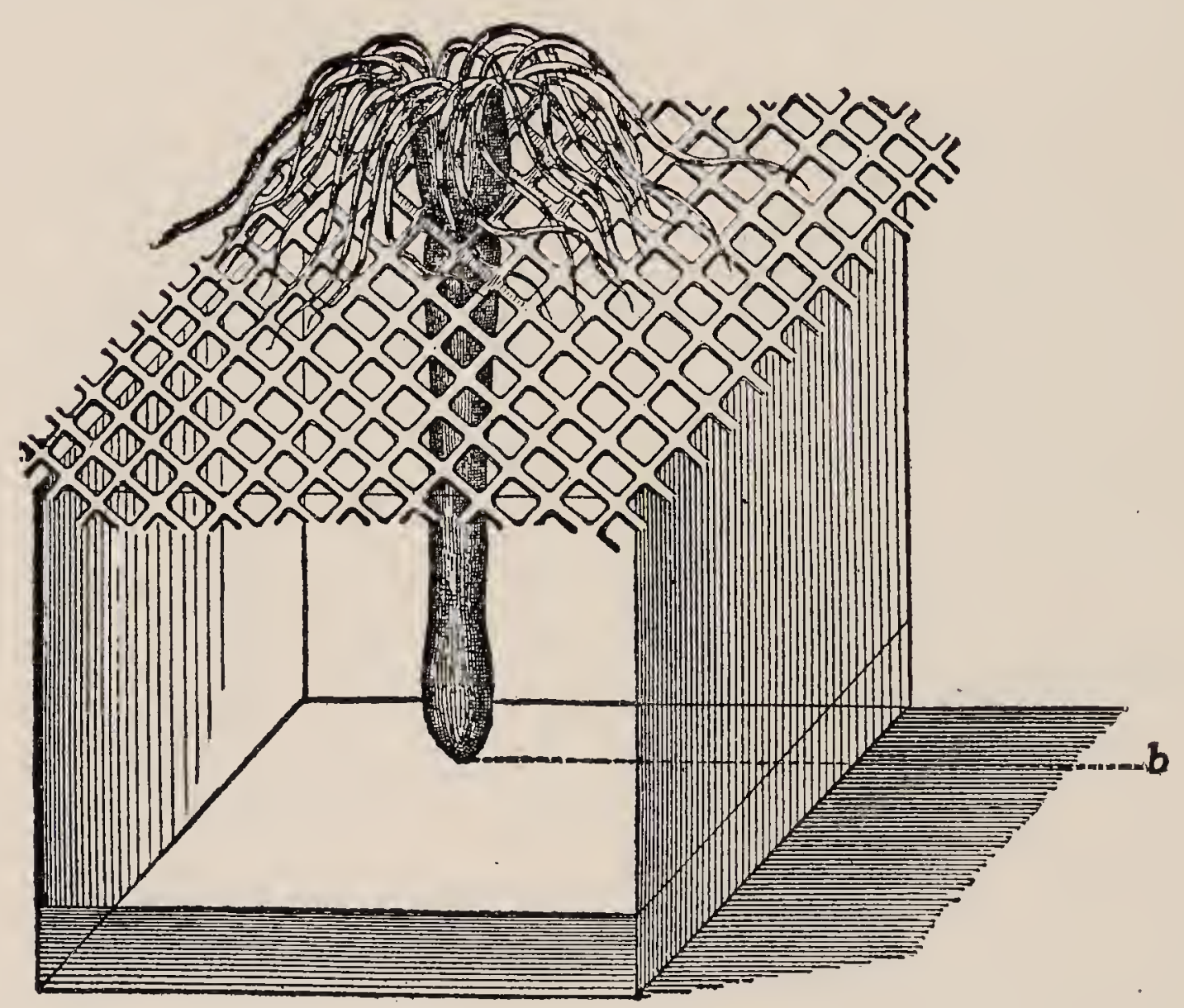

FIG. 26

The experiments were made upon Cerianthus membranaceus. The animal consists of a long, soft, and smooth cylindrical body, carrying a heavy crown of tentacles at its oral end (Fig. 26, a), while at its aboral end (Fig. 26, b) it is smooth and rounded.

The tentacles at the oral pole are arranged about the oral plate in two concentric circles; the outer circle consists of long, the inner of thin and short tentacles. In the middle of the circle is situated the oral opening, which serves also the functions of an anus. The body of the animal is hollow 
and the reader who is unacquainted with zoölogy can best picture the animal to himself by imagining a sack made up of an elastic, contractile wall, the opening of which is surrounded by tentacles.

The animals which I obtained in Naples were $5-10 \mathrm{~cm}$. long. They are very common in the Mediterranean and are especially adapted to physiological experi-

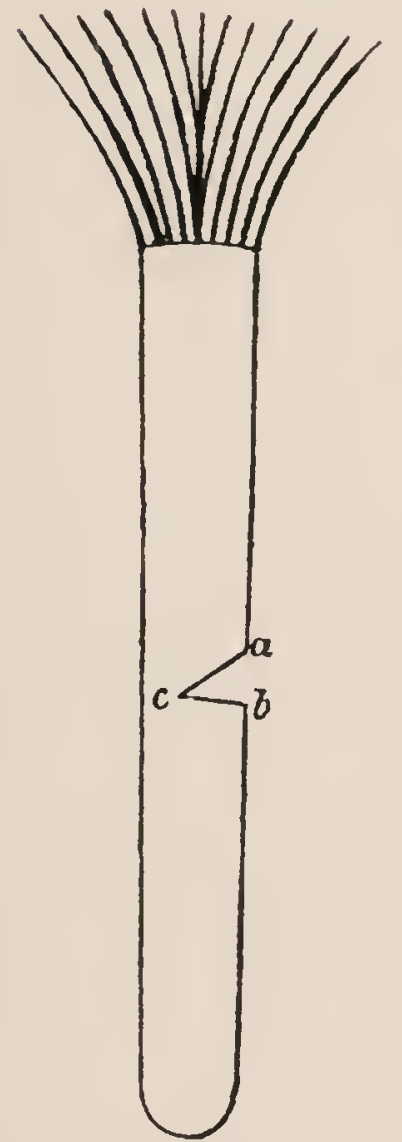

FIG. 27 ments because they are very tough and comparatively long-lived. The animal lies buried in a mucous envelope in the sand. It

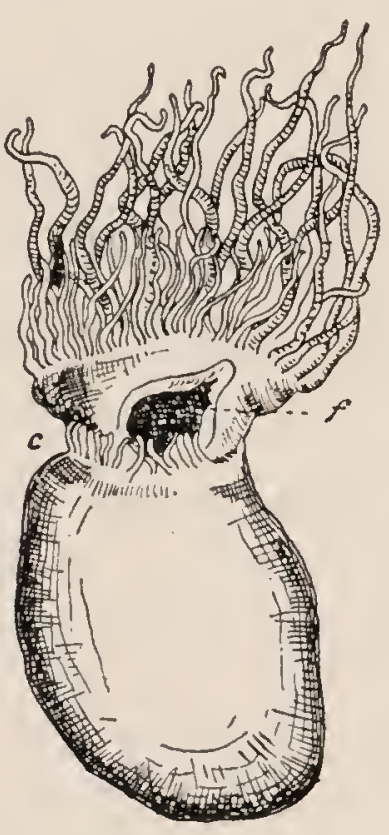

FIG. 28

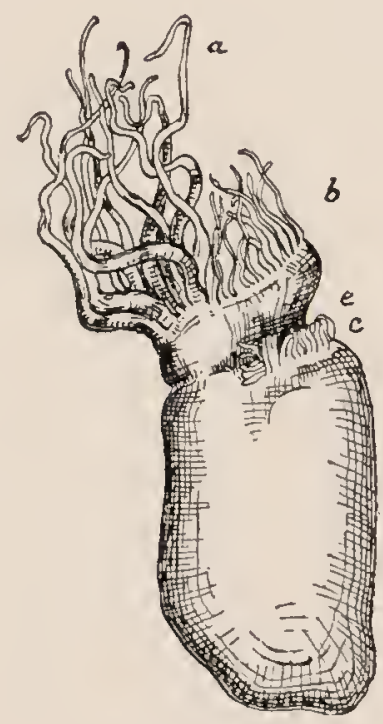

FIG. 29

only thrusts its head outside of the envelope to catch small marine animals for its prey. The envelope is formed by a secretion from the skin.

I made diagonal incisions ( $a c b$, Fig. 27) into the middle of a large number of such Cerianthi. After a few days new tentacles begin to spring from the cut surface $b c$, which grow rapidly and correspond in form, color, and marking with the tentacles at the oral pole. I have never seen even an indication of the formation of new tentacles at the other cut surface, ac. Figs. 28 and 29 represent such an animal eight days after an incision had been made. Tentacles 
spring from the lower cut surface; the upper cut surface $f$ is free from tentacles. The cut surface ac (Fig. 27) suffers changes which lead to a rounding off of this part, and make it resemble a foot. I have made more than a hundred such experiments, and yet have always obtained the same result. In order to have new tentacles form it is necessary to prevent the lips of the wound from healing together during the first few days after the operation. I attained this end most easily by laying the operated animals upon a wire screen; the animals would push their aboral ends through the meshes of the screen up to the incision. The wire then pushed itself between the lips of the wound, and so prevented the edges from healing together. First the outer row of tentacles and an oral plate were formed; then an inner row of tentacles originated; so that finally such an animal possessed two morphologically identical heads the one situated above the other. Such animals are represented in Figs. 24 and 25; $a$ is the old, $b$ the new head. The new head in Fig. 24 is about three months old; that in Fig. 25 is much younger. By similar means I also succeeded in producing animals with three heads, situated one above the other. There was nothing to prevent the production of a still larger number of heads lying one above the other, if there had been any object in doing this. I noticed that the formation of a new head and the growth of the new tentacles generally occurred more quickly and were the more considerable the nearer the incision lay to the oral pole. In animals with three heads, that lying nearest the foot had the smallest tentacles.

When the incision was made very near the aboral pole, no new head whatsoever was formed. Fig. 25 shows an animal into which I made two incisions at the same time$b$ near the middle and $c$ near the aboral end of the animal. It will be seen that new tentacles have grown from the 
incision $b$, while none have grown from the incision $c$ near the foot end, even though the lips of the wound were prevented from healing together. In the drawing which was made from life, the cut is relatively far removed from the foot end of the animal. This is because the aboral end $c d$ lying behind the second cut is greatly stretched, while that portion of the animal lying anterior to the incision is contracted. It will also be seen that after such an incision both parts become independent of each other to a certain extent; much as after transverse section of the spinal cord in one of the higher animals the incision renders the two parts of the animal comparatively independent.

3. I found no statement in the literature as to whether or not such observations had already been made upon other animals. It is, however, known that a new Hydra may spring from the body of an old one, which increases in size, and after a certain time separates from the mother to lead an individual existence. As long as it remains attached to the mother, the whole is to be regarded as an animal with two heads situated one above the other; for the body cavities of the young and the old animal communicate with each other. Yet such a Hydra is essentially different from our Cerianthus. While in Hydra not only a head, but a whole body is formed, only the oral plate is formed in Cerianthus. While the new animal becomes detached after some time in Hydra, the new head in Cerianthus remains permanently attached to the mother. Furthermore, while in Hydra the newly formed individual has the same number of tentacles, and the same cylindrical form as the mother, the number of tentacles that grow in Cerianthus is dependent upon the size of the incision. The smaller the incision, the smaller is the number of tentacles that are formed. Only a segment of a head, corresponding to the size of the incision, is therefore formed when an incision is made into a Cerianthus. A 
study of the illustrations shows that the number of new tentacles corresponds to the size of the incision, and increases or decreases as the length of the incision increases or decreases.

4. I cut rectangular pieces from the wall of Cerianthus

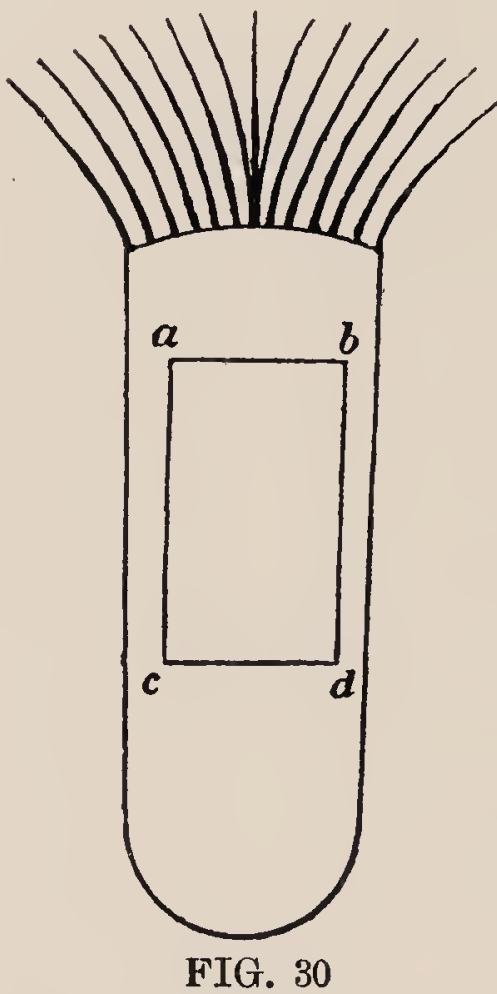

FIG. 30

by cutting off the head and the foot transversely, and dividing the resulting hollow cylinder by a longitudinal cut. These rectangular pieces formed tentacles upon only one of the four cut edges. This was upon that edge which was originally directed toward the oral pole of the animal. If $a b c d$, Fig. 30, represents such a piece, the new tentacles sprang only from the edge $a b$. The three remaining sides remained absolutely free from all evidences of new tentacles.

Thus far this experiment corresponds in behavior with that of an analogous experiment upon Hydra. ${ }^{1}$ But while the piece removed from Hydra forms a new cylinder, and a closed body-cavity before the new tentacles sprout, ${ }^{2}$ the tentacles upon the pieces of Cerianthus are formed without a new body-cavity originating, and even while the entoderm is still exposed. The cut edges that are free from tentacles may never heal together, and a new body-cavity may never

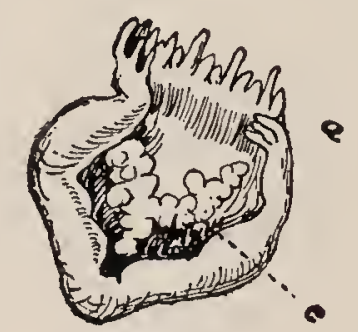

FIG. 31

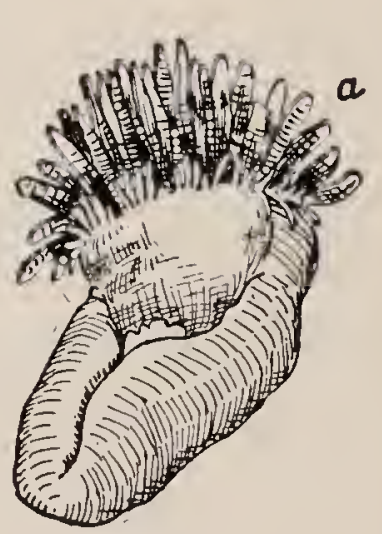

FIG. 32 be formed. In Fig. 31 is given a picture of a fragment of Cerianthus bearing large tentacles while the body-cavity is still open and the entoderm still exposed. The cut edges show inversions and puckerings, to which I shall return

1 See IschIKA WA, Zeitschrift für wissenchaftliche Zoologie, Vol. XLIX, p. 441.

2 Nussbaum, Archiv für mikroskopische Anatomie, Vol. XXIX. 
later. Fig. 32 shows the animal two months later. The cut edges have coalesced, and the animal resembles somewhat a normal Cerianthus. The inner row of small tentacles has also been formed in addition to the large peripheral ones; but - and herein these phenomena again differ from those observed in Hydra - the number of tentacles is only a fraction of the number of tentacles of the normal head, corresponding to the size of the oral cut. Nor do the ends of the two rows of tentacles meet to form a closed circle.

After what has been said, it need not be emphasized that the formation of tentacles is independent of food-supply, as the taking up of food is impossible without a body-cavity. When the pieces are too small, no tentacles whatsoever may be formed.

From all that has been said, the following observations are easily understood. When a Cerianthus is cut completely in two transversely, the aboral piece forms a new head, bearing a normal number of tentacles, while at the lower end of the oral piece, which has to regenerate a foot, new substance is deposited upon the cut surface which restores the continuity of the ectoderm at this end and assumes the rounded form of the foot.

If head and foot are both cut from a Cerianthus, the middle piece forms new tentacles at the oral and a new foot at the aboral cut surface (the latter is formed more quickly than the former). But this is possible only as long as the pieces exceed a minimal size.

5. I have tried to control the place where new tentacles are formed by contact stimuli or by orienting the animal in different ways against gravity and light. All of these experiments have thus far been unsuccessful, if for no other reason, because it was impossible to maintain the animal in any abnormal position for any length of time. The following chapter will give the reasons for this behavior. 
For the time being, therefore, the place where the tentacles in Cerianthus are formed is determined by the following law, which is only a somewhat modified expression of Allman's theory of polarity:

The place where the tentacles are formed on a fragment of Cerianthus is dependent upon the orientation which the fragment had in the uninjured animal. The tentacles always grow upon the cut surface which was directed toward the oral pole of the uninjured animal.

If for any reason, therefore, we might wish to know how a fragment of Cerianthus had been oriented in the uninjured animal, we should only have to wait until new tentacles were formed; the side upon which the tentacles sprouted would be that which was directed toward the head.

\section{RELATIONS BETWEEN FORM AND JRRITABILITY IN CERIANTHUS}

As is well known, it is possible to determine from the physical behavior of a fragment of a crystal how it was oriented in the crystal. I have tried to determine whether or not relations between body form and irritability can be shown to exist in living animals comparable to those existing between the geometrical form and the physical behavior of crystals. Such a relation, indeed, exists in Cerianthus, and this can be recognized, not only in the uninjured animal, but also in the animal deprived of its head or foot. This is true, under certain conditions, even in fragments of an animal. In this way it is sometimes possible to recognize from the behavior of a fragment toward external conditions which of its ends was originally directed toward the oral pole.

When the external conditions permit of it, Cerianthus membranaceus assumes a position in which its long axis is absolutely or nearly vertical, and in which its oral pole is directed upward and its aboral pole downward. If the head. 
or the foot of Cerianthus is amputated, or if a piece is cut out of a Cerianthus, the fragment, if not too small, and if external conditions permit of it, again assumes a vertical position, with its oral end directed upward and its aboral end directed downward. I shall now describe these phenomena in greater detail.

1. If a Cerianthus is laid upon the bottom of a vessel covered with sand, after a few minutes the foot of the animal begins to bend downward near its tip and to bore into the sand. In half an hour or less (at the proper temperature and with irritable animals) the entire animal has buried itself vertically in the sand up to its head. It remains permanently in this position, if other circumstances do not induce it to move.

2. A wire net, the meshes of which are so narrow that the body of a Cerianthus can only with difficulty be drawn through them, is supported horizontally upon a glass vessel and set into the aquarium. A Cerianthus is laid upon the wire net. After a few minutes the foot of the animal begins to turn downward, and to bore through one of the meshes of the wire net. That portion of the foot which has passed through the wire net assumes an absolutely or nearly vertical position, and remains so permanently. No change occurs at the oral pole, except that the tentacles close together so that they look like a brush, the handle of which is formed by the remaining portion of the animal. The animal crowds its body more and more through the mesh in the net, until it finally attains the vertical position shown in Fig. 26.

3. This orientation can also be reached within half an hour. But while the Actinian generally remains in the sand after having buried itself vertically in it, an animal upon the wire screen rarely retains the orientation described longer than two days; it either works itself entirely through the wire screen, or else retracts its foot to bore it through another mesh 
in the screen, or to move off the screen entirely. If, as soon as the animal has assumed the position shown in Fig. 26, the wire screen is turned over so that the foot of the animal is directed upward, the foot is not withdrawn, but begins to bend vertically downward from the tip. The bend-

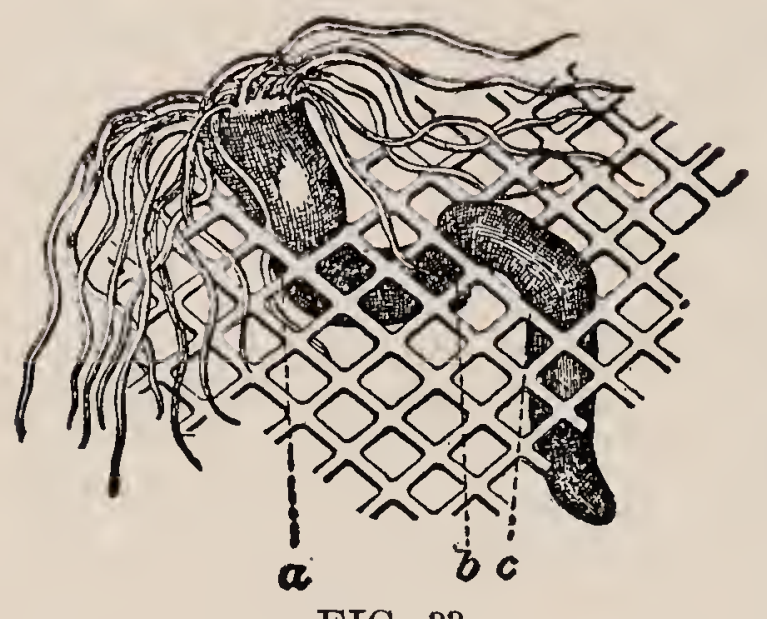

FIG. 33 ing then passes from one element of the body to the next, from the foot to the head. As soon as the tip of the foot again touches the screen, it pushes itself through it as far as possible. If the wire net is again turned over, the whole process is repeated anew. In this way the animal can be compelled, by the help of gravity alone, to weave itself through the meshes of the screen several times "of its own accord."

Fig. 33 shows a Cerianthus which has thrice passed through the meshes of the screen in this way. The drawing is taken from life.

4. Such a bending downward, which has been accurately studied in negatively geotropic roots, has never been demonstrated, so far as I know, in animals. I will therefore cite another experiment which better illustrates the course of this reaction. If a Cerianthus be put into a test-tube filled with sea-water, and the test-tube be placed so that the head of the animal is down and the foot up, while the long axis of the animal is vertical, the tip of the foot begins after some minutes to bend vertically downward. In Fig. 34 is shown the course of such an experiment. Several minutes before 12 o'clock the animal was placed in a test-tube in the position described. At 12 the foot of the animal had begun to bend downward (Fig. 34, a); in the next thirteen minutes the bending gradually advanced toward the head (Fig. 34, b). 
Five minutes later the foot reached the bottom of the testtube (Fig. 34,c). The bending spread gradually to elements lying nearer the luead; as the foot could no longer advance vertically, it was pushed horizontally over the bottom of the test-tube; and at the same time the head, which until now had played no active part, was slightly raised (2:35 P. M., Fig. 34, d). The bending then passed from one element of the body to another, until the head was brought into an erect position (Fig. 34,e). Finally the entire animal righted itself so that at 1 o'clock it had the position shown in Fig. $34, f)$. The whole righting process had therefore occupied an hour. The animal remained in this position for two days, when it crawled out of the test-tube. I have repeated the experiment many times, but always with the same result.

5. If a Cerianthus is divided transversely in the middle, and both pieces are laid upon the wire screen, they work their way (often immediately after the division) through the screen with their aboral ends directed downward. If the head and foot of an animal are amputated, the middle piece may still show this reaction. When this occurs, the aboral end always bends downward, and works its way through the wire screen. Never have I seen the reverse occurthat such an animal assumes a position in which the oral pole is directed downward and the aboral pole upward. I wished to determine whether light or gravity had any effect upon the position of the new organs formed in these headless and footless animals when fixed vertically in sand, with their aboral ends directed upward; in no case, no matter bow often I fixed the animals in an inverted position in the sand, did I succeed in retaining them in this position longer than two days. Nor did they remain with their aboral cut ends directed downward in a narrow test-tube the long axis of which stood vertically. In all cases they turned their oral poles upward. 

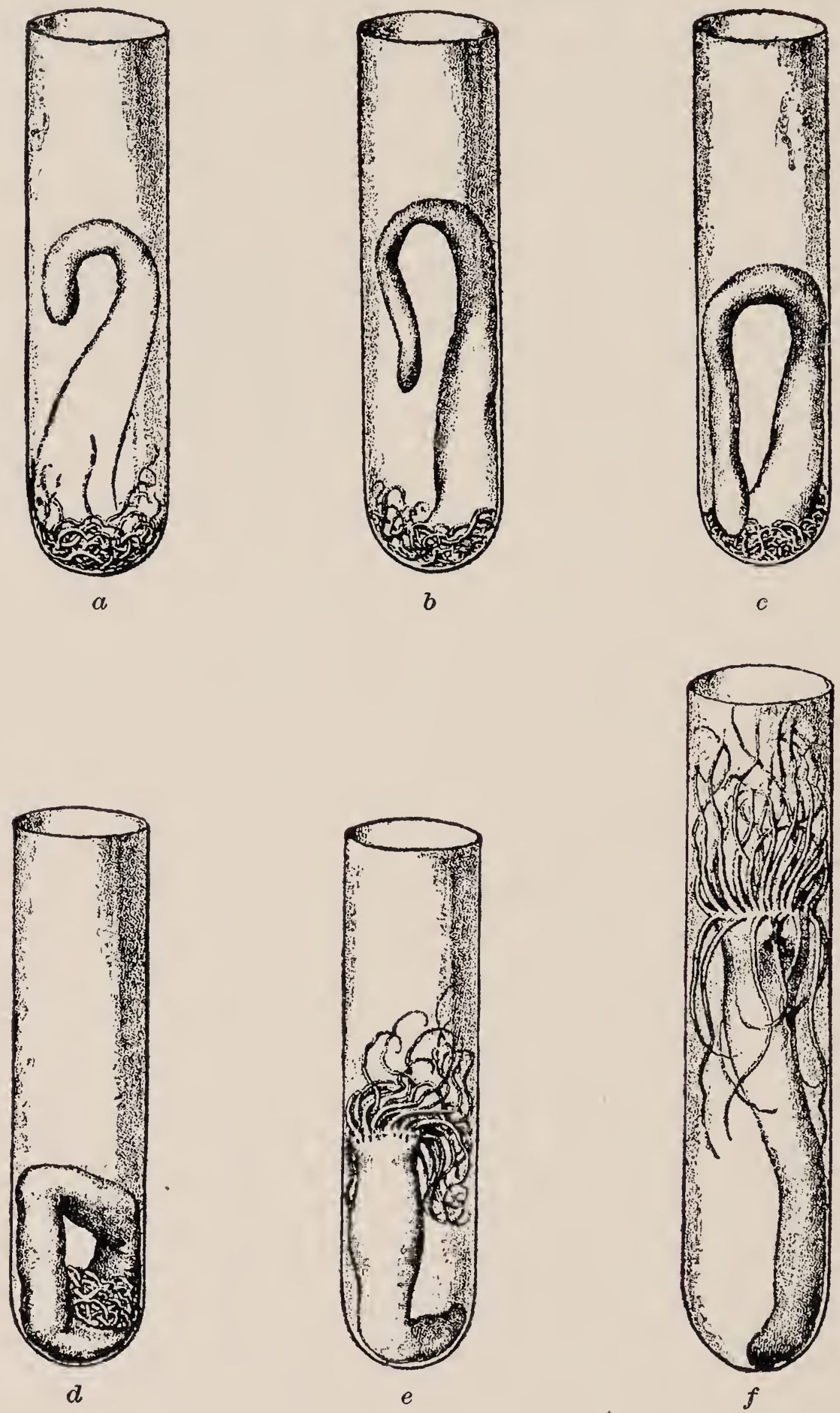

FIG. 34 
6. We have seen that in the uninjured animal it is the foot which first bends downward on the wire screen and assumes a vertical position; while the head is the last to assume this orientation. If an animal is cut across transversely, and the two pieces are laid side by side upon the wire screen immediately after the operation, the aboral fragment carrying the uninjured foot begins to bend down vertically sooner than the oral fragment which carries the head.

This difference in the irritability of the two portions of the animal can be shown very prettily by making a transverse incision at about the middle of the animal, so that both pieces still hang together. If such an animal is laid upon the wire screen, immediately after the operation, the foot works itself through the mesh in the net to the incision and assumes a vertical position, while the oral piece extending from the incision to the heads usually remains lying horizontally upon the wire screen.

7. If the heads of Cerianthus, which have been cut off close to the oral plate, and which no longer work their aboral poles through the wire mesh when laid upon it, are laid upon the sand for a time, they also at length assume a position in which their long axis is in a vertical position. One receives the impression at first that one is dealing with normal animals buried deep in the sand. The method by which they retain their vertical position is remarkable. Certain of the cells of the ectoderm secrete a mucoid substance to which kernels of sand become attached. But this secretion is formed only on the base of the pieces which have been cut off just below the oral plate. The kerneks of sand which adhere to the base have a greater specific gravity than the animal itself, and this keeps the animal in an upright position.

8. All these experiments succeed equally well in the light 
and the dark. The fact that the animal assumes a vertical position in every case seems to indicate that gravity is the determining factor. I have tried to see whether the animal would assume upon the centrifugal machine the position of one of the radii, that is, with its foot directed toward the periphery and its head toward the center of the rotating disc. But the animal had always to be kept in a vessel of water in these experiments, and the currents set up in the water by the rotation interfered with the movements of the exceedingly soft animal. Even when the animal was fastened to the wall of the vessel by a needle, its free ends were always set in motion by the water. Nothing remained, therefore, but to introduce the animal into a long test-tube which was fastened radially upon the revolving table, and to observe whether the animal directed its foot or its head toward the center of the revolving table. The experiments which have been performed thus far have not given a uniform result.

9. The animal retains a vertical position permanently only when at the same time contact stimuli act constantly upon its entire surface. The animal retains a vertical position permanently in the sand, but only for a few days at the best upon a wire screen. Iwas also able to keep the animal permanently in a horizontal position in a closely fitting test-tube. The head which projected beyond the lips of the test-tube was directed vertically upward.

How strongly these animals are compelled to bring as much as possible of their bodies in contact with other solid bodies is evidenced by the fact that they crowded themselves forcibly under lead blocks and lead plates which I had laid upon the bottom of the aquarium. This is the same form of contactirritability that is found in Forficula, larvæ of Musca, winged ants, etc. - a phenomenon which I have described in greater detail in previous publications. ${ }^{1}$

1 "The Heliotropism of Animals," p. 1, and also "Further Investigations on tho Heliotropism of Animals," p. 89. 
10. The morphogenetic polarity discussed in the preceding chapter therefore corresponds with a polarity in regard to the orientation of a Cerianthus toward gravitation. Since, however, we are as little acquainted with the structural conditions which determine the orientation of a Cerianthus as with the structural conditions which determine that the formation of tentacles only occurs at the oral end of a fragment, the question as to whether the same conditions underlie both phenomena cannot as yet be discussed.

XII. FURTHER REMARKS ON THE FORM AND LIFE PHENOMENA OF THE NEWLY FORMED HEADS IN CERIANTHUS

1. If a transverse incision such as described in sec. $x$ be made fairly close to the head, the edges of the wound do not draw together so easily. In this case new tentacles, a new oral plate, and a new mouth are formed at the oral cut edge. The part above the incision may persist for months, but finally it drops off like a wilted leaf.

If, on the other hand, the incision is made in the middle of the animal, the tendency for the edges of the wound to heal together is very great. New tentacles (external and internal) and a new oral plate are formed; but never has a mouth formed in any of the cases observed thus far. The newly formed head was therefore of no use whatsoever to the animal. If we look more closely at such a head (Fig. 24,b), the ectoderm is seen to pass over into an oral plate at $b$, which is covered with two rows of tentacles. An opening no longer exists in the ectoderm.

We saw, moreover, that quadrangular pieces cut from the wall of a Cerianthus formed tentacles upon one side only. A second circumstance to be considered is the fact that the elastic tension of the inner layer of the wall is greater than that of the external. In consequence of this, the three remaining cut edges, upon which no tentacles are formed, 
roll inward, so that only the ectoderm is visible externally (Fig. 35). Because of these mechanical conditions the part assumes after some time-especially when the new tentacles begin to grow - an appearance which reminds one in some ways of a normal Cerianthus. Of course, many pieces remain permanently monstrosities. So far as the experiments performed hitherto are concerned, a mouth has never been formed in these pieces. This is a remarkable fact, and seems to indicate that the animals have a source of food-supply which

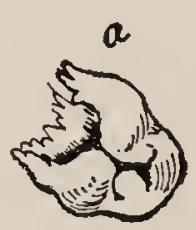

FIG. 35 differs from that of the uninjured animal, for they remain alive, and do not diminish markedly in bulk even in the course of months.

2. These mouthless heads when brought in contact with food reacted exactly as normal heads. The reader is probably acquainted from personal observation with the behavior of an Actinian when a piece of meat is laid upon the tip of one of its tentacles. The tentacle becomes concave toward the piece of meat, winds itself about the meat-as a vine about a support-and finally bends so that the piece of meat reaches the middle of the oral plate, where the mouth is situated in normal animals. In Cerianthus the inner tentacles then fold over the meat; some or all of the external tentacles then follow in a similar way, and it looks as though the tentacles were pressing the meat into the mouth. The meat reaches the stomach, and the tentacles then unfold. But this reaction is certain to occur only when the substance laid upon the tentacles has certain chemical and mechanical characteristics. If a grain of sand is laid upon the tentacles instead of the meat, the tentacles do not bend in as described.

If a piece of meat is carefully laid upon the tip of the external tentacles of a newly formed head, which has no oral opening, they also seize it in the manner just described; 
they carry it to the middle of the newly formed oral plate, and the inner tentacles cover the meat and press it against the oral plate; the outer tentacles then also cover the meat, and the animal struggles several minutes in vain to press the meat into a mouth which does not exist. The external tentacles are then withdrawn from the center of the oral plate and expanded, and the same is done with the internal tentacles. The piece of meat agrin reaches the edges of the tentacles (probably through ciliary motion) and drops off.

The experiment can be repeated with the same result any number of times upon the newly formed heads which have no oral opening; they always react when a piece of meat is laid upon the tentacles. No trace of memory is present.

In order to have the new head react with certainty, it is necessary that the substance laid upon the tentacles have the same characteristics as that necessary to call forth the reaction in the old head. Pieces of meat are always carried to the center of the oral plate by the tentacles of the new head, but this does not occur when kernels of sand are used. If the head of a Cerianthus is amputated, the animal does not again take up food until the new mouth has been formed and the tentacles have attained a certain size. We shall see that other Actinia behave differently in this respect.

3. The fruitless attempts of the mouthless heads to take up food is somewhat comical in the light of an optimistic teleology. The physiologist, however, takes it for granted that the tentacles of the mouthless head must react in a similar way to chemical and mechanical stimuli as the tentacles provided with a mouth, because they have the same external form, and possibly also the same structure. That the meat is finally brought to the mouth through these reactions (bendings), and that when the meat has reached the mouth the bendings again are reversed, does, of course, not influence the immediate effect of the contact between ten- 
tacles and meat; just as a moth must react to a flame with progressive heliotropic movements, even though it afterward derives no actual benefit, but actual harm, from this sort of reaction.

4. Cerianthus remains permanently in one place if its body is in contact with solid bodies and if it is properly fed. If the feeding is interrupted, it occasionally leaves its tubes in the sand to burrow anew after some time in some other part of the sand. If, however, the head of the Cerianthus is amputated, this otherwise sessile animal becomes a complete nomad. It burrows, remains for a few hours in its tube, crawls out again, buries itself anew in some other place in the sand, only to leave its new home after a short time, etc. When the tentacles have again grown, the animal becomes more sessile again.

XIII. THE IMPORTANCE OF TURGOR FOR THE GROWTH OF THE TENTACLES IN CERIANTHUS

1. Although the analysis of the mechanical conditions which influence the growth of plants has made great strides, a physiology of animal growth does not exist even by name in the modern text-books of animal physiology. It may therefore be permissible to describe here a very simple experiment which shows that one of the fundamental conditions necessary for the growth of vegetable tissues - turgormust be fulfilled in animal tissues also in order that growth may occur.

It may be known to the reader that the growth of plants decreases or entirely stops when they wilt, but that it increases when a plentiful supply of water is at hand. It is believed that the cell contents of the growing part of a plant take up water energetically from their surroundings (due to the salts of the organic acids contained in them). In consequence of this absorption of water, the cell-walls 
are stretched. This stretching of the cell membrane permits the deposition of new material in the cell-growth. When the hydrostatic pressure in the cells of an organ attains a height in which the cell membrane is tensely stretched, the organ is said to be turgescent.

In the course of the experiments detailed in the preceding chapter I accidentally discovered a means by which the turgor of a part of the tentacles of a Cerianthus can be diminished, while in the others it remains unaltered. I used this method to determine whether a diminution in the turgor would decrease or stop the growth in animal organs. If a transverse incision is made into the body of a Cerianthus such as is necessary to cause the growth of a second head, the incision has a striking effect upon the behavior of the tentacles. If one watches such an animal when its tentacles are stretched out, it is seen that those tentacles which are situated above the incision are distinctly, often as much as one-half, thinner and shorter than the remaining tentacles. This difference is shown distinctly in Fig. 29 which represents the same animal as Fig. 28, only viewed from another side. This difference in the turgor of the tentacles is permanent when the incision is made near the oral plate, and when the edges of the wound are not allowed to heal together. As soon as the wound heals, the turgor of the tentacles is re-established. If the irritability of such wilted tentacles is compared to the irritability of the turgescent tentacles of the same animal, it is found that the irritability is not markedly changed during the first few days after the incision.

If a piece of meat be carefully laid upon the tip of such a wilted tentacle, it is carried to the mouth in the same way as by an erect tentacle. Only it seemed to me that the movement of the wilted tentacle was slower and more awkward than that of the turgescent tentacle. 
The explanation is sometimes given that the tentacles of Actinia are stretched by a contraction of the muscles of the body-wall which forces water out of the body-cavity into the hollow tentacles. The turgor of the tentacles of Cerianthus cannot well be brought about in this way; for, if this were the case, the turgor of all the tentacles would have to be decreased when the body-cavity is opened; but only the turgor of the tentacles above the incision is diminished, while it remains the same in the others.

2. I amputated the heads of a large number of Cerianthi. After some time - which was, within certain limits, shorter as the temperature of the water was higher-new tentacles were formed at the cut edge. I waited until the newly sprouted tentacles had reached a length of $5-10 \mathrm{~mm}$. when stretched out. I then made a partial transverse incision into the body and prevented the wound from healing together. The tentacles above the incision lost some of their turgor, and ceased to grow from that time on. The remaining tentacles, however, continued to grow and after several weeks reached a length of $30 \mathrm{~mm}$. or more when stretched out.

As I had to bring my experiments to a close, I could not determine whether the wilted tentacles could again be made to grow by restoring their turgor. I hope to be able to make this and further experiments on growth at another time.

The fundamental condition for growth in plants is therefore also found in animals.

XIV. ON THE EXTERNAL CONDITIONS WHICH DETERMINE THE FORMATION OF TUBES IN CERIANTHUS MEMBRANACEUS

If a Cerianthus is laid upon the sand, and a sufficient time is allowed the animal to burrow, it is noticed after several days that the hole in which it lies is covered with a 
smooth coating. If the Cerianthus is pulled out of the sand, it is found to be incased in a soft tube which is smooth internally and covered on the outside with fine grains of sand. The animal can be drawn out of the tube without injury. It seems very human that the animal should arrange itself comfortably in the sand and protect itself against unwelcome visitors by building a tube about itself. This formation of tubes by Cerianthus appears to belong to those cases to which the old phrases of "instinct" and "artistic impulse of animals" might be applied. It may perhaps be of interest to some of the readers to become acquainted with the following simple experiments which show upon what foundation anthropomorphic conceptions of life-phenomena are occasionally based.

1. If a Cerianthus is carefully drawn out of its tube and laid upon a very thin layer of sand in which it cannot burrow, a secretion is soon formed at the surface of those portions of the body which rub against the sand. The surrounding particles of sand stick to this secretion. The continued movement of the animal, and the propagation of stimuli from those parts of the animal which are rubbed in the sand to neighboring parts of its surface, cause the secretion to be poured out over the entire surface of the body of the animal. The tube is then completed. It is at first very thin, but becomes thicker in the course of time, as more secretion is poured out in consequence of the continued friction. According to these observations, therefore, the entire process of "tube-building" is nothing but a process of secretion, the stimulus for which is found in the friction of the surface of the body against solids. This is confirmed by the following experiments.

2. The thickness of the tube is dependent upon the degree of friction. If one Cerianthus is laid upon sand, while another is introduced into a carefully cleaned test- 
tube, the former constructs a firm tube of mucus in the course of a few hours, while the latter which is kept in a test-tube, and the skin of which encounters but little friction from the smooth glass, forms a scarcely perceptible veil in the course of twenty-four hours. The greater amount of friction brings about a greater secretion and a more extensive tube-building.

3. I fastened a Cerianthus to the underside of a cork floating at the surface of the aquarium. The Cerianthus was fastened to the cork by passing a pin through its body. The head and foot of the animal hung down loosely upon either side of the pin. I waited four weeks, but no membrane was formed upon the parts which did not come in contact with solid bodies. But a secretion occurred at those places where the Cerianthus rubbed against the cork or the head of the pin. The mass of mucus secreted at these points attained the thickness of a finger in four weeks.

The wound occasioned by the pin was not the cause of this secretion, but only the friction, for I observed the same phenomena in uninjured Cerianthi which remained for some time in the meshes of a wire screen. Only in the latter case it is very difficult to keep a Cerianthus very long in this position without movement.

The formation of a tube by Cerianthus offers therefore the same evidence of "artistic impulse" as the secretion of saliva during mastication.

\section{EXPERIMENTS ON ORGANIZATION AND IRRITABILITY IN SOME OTHER ACTINIA}

1. I have made experiments similar to those upon Cerianthus on the determination of the situation of the new head in a number of other Actinians-Actinia équina of the Bay of Naples and the East Sea, Actinia cari, Adamsia Rondelletti, Anemonia sulcata, Cereactis aurantiaca, etc. 
I always found that new tentacles were formed only upon the oral edge of the piece cut from the animal, while a new foot was formed only at the aboral end. Even though I have not thus far been able to cause a head to develop at the aboral end in Actinia as in Tubularia, still I consider it probable that this also will succeed because of a note I found in Contarini's 'Trattato delle Attinie, ${ }^{1}$ according to which Diquemare, ${ }^{2}$ who worked on regeneration in Actinia, once noticed such a heteromorphosis. A piece cut transversely from an Actinian "riprodurre in vece di un piede degli altri tentacoli e un altra bocca, cosi mangiara da due parti nello stesso tempo.",

2. In these experiments the Actinia equina of the East Sea ${ }^{4}$ behaved in some respects differently from Cerianthus. When I cut transverse pieces from Actinia equina, they formed tentacles only, and these without exception at the oral pole; I never saw a new foot formed upon transverse pieces of this animal. The wound only healed at the aboral pole; no regeneration whatever occurred here, and the bodycavity of such an animal communicated with the outer world at both poles. Most remarkable, however, was the fact that both poles took up food, the aboral mouth being even more voracious than the normal mouth at the oral end. Substances were swallowed by the aboral mouth which the oral mouth as a rule does not take up. If a paper wad soaked in sea water is laid upon the normal mouth of an Actinia equina of the East Sea, it is not swallowed; while a piece of crab meat, which by our tongue cannot be distinguished from the taste of the paper wad, is immediately taken up by the animal. I tied a paper wad to one end of a stout thread

1 N. Contarin, Trattato delle Attinie (Venezia, 1844).

2 I have not becn able to obtain Diquemare's work.

3 Very recently Professor Torrey, of the University of California, has observed heteromorphosis in an Actinian. [1903]

${ }^{4}$ The Actinia equina of the East Sea is not identical in its physiological behariof with the Actinia equina of the Bay of Naples. 
and a piece of meat to the other, and then threw the whole upon the outstretched tentacles of a hungry animal. The tentacles which came in contact with the meat reacted at once by bending by which the meat was carried to the mouth; the tentacles in contact with the paper did not react. I removed the thread and reversed the position of the meat and paper upon the oral plate, so that the tentacles which had before been touched by the paper were now in contact with the meat. The tentacles touched by the meat carried it to the mouth, while the tentacles touched by the paper let it fall. The meat was then crowded into the mouth, and the thread was pulled in after it; but the paper and a piece of the thread remained outside of the oral opening. No change occurred within the next twenty-four hours. After this period the thread was ejected, but without the meat. The latter had probably been digested. I have often repeated this experiment with the same result; only occasionally the thread was ejected earlier, and then a piece or all of the undigested meat was still attached to the thread.

I divided an A. equina into two pieces by a transverse incision. The oral piece - which I shall call the head piece - had the old normal mouth at its oral end; the body-cavity was open also at the aboral end of the head piece, and food was taken up here likewise, even though no tentacles were present. The old oral mouth of the head piece showed the same choice in the taking up of food after the division of the animal as before. But the aboral mouth of the head piece at times took up paper wads and swallowed them. Yet I saw it refuse paper wads while at the same time it eagerly took up pieces of crab meat.

While the old mouth at times refused meat, the aboral mouth was nearly always ready to take up food.

3. The following means, however, served to decrease the irritability of the mouth toward chemical and mechanical 
stimuli. When I stood the head piece upon its oral mouth for but one hour, so that the mouth was in contact with the bottom of the vessel, the animal would take up no food for a long time (often as long as twenty-four hours) when again turned over. By similar means I could bring about the same effect at the new aboral mouth. But it was necessary to keep the animal with its aboral mouth downward a much longer time - half a day perhaps — and then the effect lasted only an hour. In this experiment it cannot be that every abnormal stimulus simply inhibits the irritability of the mouth. For a series of artificial mouths made by transverse incisions into an animal ate meat despite the wounds, immediately after the division of the animal. The (transitory) loss of the specific irritability of the mouth is probably due to the contact stimuli which act upon the mouth.'

4. I laid pieces of Actinia which took up nourishment at both ends upon the side and tried to see whether both mouths would take up food at the same time. I first held a large piece of meat against the aboral mouth, which was, as usual, tightly closed in consequence of the contraction of the circular muscle fibers. The meat caused the mouth to open and to seize and slowly crowd it into the body-cavity. Before the piece of meat had been entirely swallowed I offered another piece to the oral mouth. This was also taken up. At the same moment the act of deglutition was interrupted at the other mouth by a firm contraction of the ring muscles. After a few moments, when the meat had been crowded into the oral mouth, the musculature at the aboral end relaxed and the piece of meat dropped out of the mouth. When I fed the two mouths successively, that which had been fed first gave up its food when the other began to take up its food. Peristaltic waves seemed to pass frequently in both directions in these animals in which the body-cavity

1 Or the lack of oxygen. [1903] 
is opened at both ends. The meat after being swallowed was often ejected undigested. Actual nutrition of these animals was, of course, impossible.

5. We have thus far considered only the head piece of a divided Actinian. The foot piece which possesses an uninjured foot at its aboral end, and a cut edge at its oral end, soon regenerates tentacles at this end and a normal mouth is formed. But even before the tentacles and mouth have begun to regenerate, the oral opening assumes the functions of a mouth. Pieces of meat are taken up and swallowed, while I have never seen it take up wads of paper or grains of sand.

6. While the entire body of Cerianthus, with the exception of the oral plate, is endowed with contact-irritability, this contact-irritability is confined to the basal surface of the body of Actinia equina. By means of this surface the animal attaches itself to solid bodies. The surface of the foot has here the same function as the roots of Tubularia, only with this difference, that through (spontaneous) internal changes the Actinian can again leave the surface of the body to which it is attached, while this is not possible in Tubularia. It is interesting to note that the nature of the surface of the solid body is not a matter of indifference in calling forth these reactions. When no other object was near, the animal attached itself to the glass wall of the aquarium and slid about upon it; when, however, I placed the shell of a Mytilus in the aquarium, and the animal came in contact with it in the course of its movements, it immediately attached itself to it and remained there, it mattered not whether the shell was empty or occupied.

The surface of an ulva leaf in the aquarium acted in the same way as the surface of the mussel. While the animal would always leave the glass to which it was fastened, to attach itself to an ulva leaf when brought in contact with it, 
the reverse phenomenon-namely, that the animal would leave the ulva leaf or the mussel shell to attach itself to the glass-occurred but rarely. This contact-irritability of the foot does not change when the head or the larger portion of the oral part of the animal is amputated. I kept the oral end of such a piece of an animal in contact with the bottom of the aquarium, while its foot extended upward. A slide was placed in contact with the foot, to which the animal might easily have attached itself, but this it did not do. As soon, however, as an ulva leaf floating in the aquarium came in contact with the foot, the animal attached itself to it immediately.

7. The aboral pieces of transversely divided Actinia which still had a foot remained alive longer than the oral pieces. The latter usually succumbed to a fungus disease after a few weeks. The disease began ordinarily in the tentacles which had lost their turgidity after the operation. Contarini seems to have made a similar observation in operations on Actinia (Aiptasia) diaphena.

\section{SUMMARY OF RESULTS}

In conclusion I wish to summarize briefly the chier results of these investigations.

I. We saw first of all that there are certain animals in which it is possible to control the place where an organ is formed by external conditions, in such a way as to substitute in place of a lost organ one which differs from it in form and function (heteromorphosis). It is thus possible to produce at will forms with normal vitality, which differ from the hereditary forms produced by nature in a definite way. In detail the heteromorphoses thus far accomplished are the following:

1. In Tubularia mesembryanthemum:

a) If a piece of stem, which must not be below a certain 
minimal size, is cut from Tubularia mesembryanthemum, and both its ends are surrounded by water, a head is formed at both ends (Fig. 16). The head is usually formed more rapidly at the oral than at the aboral end. In this way it is possible to produce any number of bioral animals without interfering with their vitality.

b) If a piece of root is carefully separated from the base to which it was attached, so that it is surrounded on all sides by water, a head is formed at its aboral end. The root continues to grow but forms no polyps if it is allowed to attach itself anew as a root.

2. If a portion of the stem is cut from Aglaophenia and suspended vertically in the aquarium, it always forms a root at its lower end, according to observations made thus far; it matters not whether the apical or the basal end is directed downward. Either a tip or a root is formed at the end directed upward (toward the zenith), but a tip is formed the more readily when the apical end is directed upward.

It is therefore possible to create biapical and bibasal forms (Figs. 17 and 18) in Aglaophenia; yet the certainty with which bibasal animals can be created is greater than that with which biapical animals can be produced.

3. If the stems of Plumularia pinnata are cut off close to the root and fixed in a vertical position, but with the tip downward, a new tip instead of a new root may arise from the basal end, which continues to grow upward; more frequently a root first springs from this end, from which arises a stem that grows upward (Fig. 20, $a, b$ ).

$4 a)$ If a piece is cut from the stem of Eudendrium, and both ends are surrounded by water, new tips are formed at both extremities (Fig. 21, $a, b$ ). Yet a variation occurs at times which $\mathrm{I}$ have not observed in the beforementioned animals; namely, a new tip and a new root may grow from the same cut end. 
b) It was possible to cause the growth of roots in the middle of a stem by bringing this point in contact with solid bodies.

5 ( $\iota)$ It was possible to grow tips from the basal cut end of a stem of Sertularia polyzonia by fixing the stems in the aquarium with their basal ends upward (toward the source of light). New roots were usually formed at this end in addition to the new tips (Fig. 22, $a, b$ ).

b) So far as my present experiments go, new branches were formed only on that side of the old stem or root which was turned toward the light.

6. It was possible to produce biapical animals in Gonothyræ Lovenii.

II. In a long series of animals, particularly Actinians, I have not yet succeeded in causing a heteromorphosis of any kind. In these animals the position of the regenerated organ is determined (as far as our present knowledge goes) by the orientation of the fragment occupied in the uninjured organism, a new head is formed at the oral edge of a piece of such an animal, while a new foot is regenerated at the aboral end. This law governs regeneration, not only in Actinia, but also in Hydra, in certain starfish which I have studied, in a series of worms, snails, crustaceans, and animals stili higher in the scale.

III. The same behavior in organization is therefore found in the animal kingdom as in the vegetable. As is well known, it is possible to control the position of a new organ (just as in the animals given under heading I) in certain plants, while in others (as in Actinians) regeneration is dependent upon conditions which are at present unknown, and are apparently internal.

IV. The following are mentioned as some of the different forms of irritability which influence the orientation of animal organs : 
1. Contact-irritability (stereotropism).

a) In a series of Hydroids the root attaches itself to the surface of a solid body as soon as it comes in contact with it, and continues to grow over its surface, adhering to it as closely as possible.

The stems of these Hydroids do not possess this irritability. In Tubularia, in which the polyps are large enough to permit one to experiment upon them, it can be shown that they possess the opposite kind of contact-irritability. When brought in contact with the surface of solid bodies they bend away from it. By taking advantage of this irritability of the polyps, it is possible to bring about permanent (stereotropic) curvature in the Tubularian stem.

b) Only the tip of a root which is growing attaches itself to the surface of a solid body.

c) In order that the root may attach itself it is necessary that the contact stimulus should act for some time.

d) It has already been mentioned that contact-irritability can cause the growth of roots in the middle of a stem-for example, in Eudendrium.

e) When in a number of Hydroids stems arise from the roots which have become attached to a solid body, these new stems originate on that side of the roots which lies diametrically opposite the solid body.

2. Heliotropism.

a) In Sertularia (polyzonias?) the branches are positively, the roots negatively, heliotropic. Only the growing parts show any heliotropic curvatures.

3. Geotropism (?).

a) When possible, Cerianthus membranaceus always assumes a position in which its long axis is vertical, its oral pole upward, and its aboral pole downward. If the animal is placed in a different position, the foot tries to gain its normal orientation by bending vertically downward. 
b) The main root and the adventitious roots of Aglaophenia, when they do not come in contact with the surface of solid bodies, bend downward and continue to grow in this direction; while the stems bend upward, grow toward the zenith, and arise from the upper surface of a horizontally growing root.

$\mathrm{V}$. The following phenomena are of importance in the general physiology of animal growth :

1. For the growth of the tentacles of Cerianthus, as for the growth of plant tissues, it is absolutely necessary that the hydrostatic pressure in the cells of the organ exceeds a certain amount (that the organ is turgescent).

2. The growth of the roots of Aglaophenia, Sertularia, and other Hydrozoa occurs only in a small region near the tip of the roots as is the case in the analogous plant tissues.

3. When the roots of Aglaophenia, Gonothyræa, Plumularia, and Sertularia become attached to solid bodies, they grow in length much more rapidly, and their absolute growth is much greater than when they are surrounded on all sides by water. This has already been demonstrated by Dalyell in other. Hydrozoa.

VI. Of the special results the following only may be mentioned: If a transverse incision is made into the bodywall of Cerianthus near the oral plate, only those tentacles situated above the cut lose their turgidity, while the remaining tentacles retain theirs. The turgidity can therefore not depend upon a contraction of the body-wall which forces water into the tentacles. 


\section{V \\ GEOTROPISM IN ANIMALS ${ }^{1}$}

\section{GEOTROPIC CURVATURES IN ANIMALS}

As a continuation of observations which I have already published $^{2}$ I wish to give in the following pages some further facts which show that certain animals are compelled to orient their bodies in a definite way toward the center of the earth, as are certain plants.

In order to show more clearly the similarity between the behavior of animals and that of plants in this respect, I quote the following passage from Sachs on geotropism in the plant kingdom:

Whenever portions of a plant are moved by any cause whatsoever from their habitual position into a different position with reference to the horizontal, they bend until they again assume the same relation with the horizon which they had originally. This bending, which is brought about through the mere change in position, is the effect of a geotropic stimulus, the consequence of some property of the organs which does not give them any rest until they are again at their proper angle with the direction of the force of gravitation. ${ }^{3}$

These geotropic bendings of plants, as Sachs adds, "are brought about exclusively through growth, and only those organs which are still capable of growth can therefore regain their normal position with reference to the horizontal."

I have pointed out in an earlier paper that the roots of Aglaophenia pluma, a Hydroid, have the tendency to grow downward. Curvatures at the same time take place in this animal which are determined by internal causes, and which

1 Pflügers Archiv, Vol. XLIX (1891), p. 175. and 89.

2 Sitzungsber. der Würzburger physik.-med. Gesellschaft, 1888, and Part I, pp. 1

3 J. SACHs, Vorlesungen über Pflanzen-Physiologie, 2d ed. (Leipzig, 1887), p. 717. 
somplicate the phenomena of geotropism. Recently, however, I have discovered geotropic bendings in the growing portions of a different Hydroid (Antennularia antennina) which are not masked by any secondary phenomena.

Antennularia antennina consists of a main stem about $1 \mathrm{~mm}$. in diameter, and often $20 \mathrm{~cm}$. long, which usually arises perfectly perpendicularly from a felt-like mass of very fine rootlets. From the main stem spring in regular order very delicate, short, unbranching lateral twigs, upon the upper surface of which are found polyps and nematophores. If such a stem is placed in any position deviating from the vertical, the tip of the stem, if it grows at all, bends sharply back toward the vertical and continues to grow vertically upward. Only the newly growing portion of the tip is able thus to change its orientation. If the orientation of the animal is not again altered, the stem grows absolutely vertically upward; it is negatively geotropic. The roots, upon the other hand, grow vertically downward; they are positively geotropic; yet the direction of their downward growth is not so perfectly straight as that of the upward-growing stem. As often as the orientation of the stem with reference to the vertical is changed, if any new growth whatsoever occurs, the stem bends toward the vertical and grows upward in this direction.

But not only the orientation of the organs, but also the place where new organs originate, is dependent to a large extent upon gravitation. But I will speak of these facts at another place, and publish therewith the pictures necessary to illustrate them.

These curvatures during growth are independent of the light. They。ocur equally well whether the stems are grown in the dark or the light room; when cultivated in the light, so far as I have been able to see, their orientation is not affected in the least by the direction of the rays of light. 
Other conditions than light and gravity which might have influenced the perfectly vertical growth of the stem, or its geotropic bendings, when its position with reference to the vertical was altered, were shut out in these experiments. I was not able to make any experiments upon the centrifugal machine, as it takes some twenty-four hours to bring about the geotropic bendings, and because $I$ could avail myself of no motive power which ran uninterruptedly for so long a time. There can be no doubt, however, that we are dealing in this case with a geotropic phenomenon.

There are, on the other hand, animals which are capable of geotropic curvatures through muscular contractions without any accompanying phenomena of growth. We find such conditions in an Actinian, Cerianthus membranaceus. This animal has the habit of burying itself vertically in the sand. If the animal is brought into any other orientation toward the vertical, it bends its body downward, beginning with the foot, until the entire animal has again a vertical position. ${ }^{1}$

\section{GEOTROPISM IN FREE-SWIMMING ANIMALS AND ITS SIGNIFICANCE FOR THE BATHOMETRIC DISTRIBUTION OF CERTAIN PELAGIC ANIMALS}

1. Since the geotropic effects in the vegetable kingdom have been studied, in the main, only upon sessile organs, and are known in these only in the form of curvatures during growth, objection might perhaps be taken to the fact that I intend to speak of geotropism in free-swimming animals. But the term "geotropism" signifies only a dependence of the orientation upon gravitation, without saying anything concerning the mechanism of this dependence. It would therefore be mere pedantry if we should declare that such dependence could be designated geotropism only in sessile and not in motile animals. I will lose no time in arguing this question,

1 Part I, p. 155. 
and rather quote the words with which $\nearrow$. Sachs describes the geotropism of motile plants:

I have already called attention to the remarkable way in which Plasmodia crawl up the stems of plants, flower-pots, and other comparatively high objects. They can be induced to do this most readily when moist glass plates are fixed vertically into tan bark containing young Plasmodia which are just ready to creep to the surface. In the course of a few hours the mesh-like bodies crawl to the highest points of the glass plate, which can now be removed and observed directly under the microscope in order to watch their movements more accurately. It can scarcely be doubted that this impulse to crawl upward is to be considered the effect of a geotropic stimulus; that is to say, that an as yet unknown effect of gravitation upon the molecular structure of the protoplasm influences the movements of the molecules in such a way that the effect which we have described is finally brought about. It is scarcely necessary to add that the individual mechanical factors which play a rôle in this process are entirely unknown. ${ }^{1}$

Others dispute the idea that the Plasmodia are geotropic. They claim that these phenomena are the expression of a rheotropism and hydrotropism.

I showed in one of my earlier papers that certain insects behave in a way analogous to the movements of Plasmodia. In the case of insects these movements are certainly not dependent upon rheotropism and hydrotropism. If certain insects, such as Coccinellæ for instance, are introduced into a closed wooden box (and are in addition kept in a dark room in order to shut out every effect of light), they have a tendency to creep up the vertical walls of the box and to collect in the highest regions. The behavior of these animals toward gravitation corresponds with that which Sachs has described for Plasmodia.

A similar phenomenon is noticed in marine animals. In this connection it contributes toward the understanding of bathometric distribution of these animals. The reader is

1.J. SACHS, op. cit., p. 639. 
undoubtedly familiar with the fact that many marine animals are found only at certain depths in the ocean. Thus far it has not been determined what conditions compel these animals to behave in this way, Groom and I together have shown how the heliotropism of certain pelagic animals must lead to periodic depth-migrations. ${ }^{1}$ I can show by several examples that gravitation is also of importance in the distribution of pelagic animals at various depths, and that it is this force which compels certain animals to live in the surface regions of the water.

Everyone who will watch the animals found about the rocks or piles near the surface of the ocean during a quiet sea will notice the relatively large proportions of Echinoderms. Some of these Echinoderms - such, for example, as Cucumaria cucumis which is found in great numbers in the Bay of Naples - live near the surface of the water, or not more than $30 \mathrm{~m}$. below it. It can be readily shown, however, that Cucumaria cucumis is, like the Plasmodia or the Coccinellæe, compelled to crawl upward on vertical surfaces, and that apparently gravitation determines this behavior. Cucumaria cucumis has an elongated pentagonal body some 10 or more cm. long, carrying at its oral pole radially arranged arborescent tentacles. Upon each of the five edges are found two parallel rows of tiny feet by which the animal is enabled to crawl upward, even upon smooth glass plates. If these animals are introduced into an aquarium, they creep about the bottom until they reach a vertical wall, up which they climb to remain at its highest point, and when possible immediately under the surface of the water. The animal remains permanently in this position and behaves like a sessile animal.

If such a Cucumaria cucumis is permitted to attach itself to a vertical glass wall which can be turned about a hori-

1 Groom und Loeb, Biologisches Centralblatt, Vol. X (1890). 
zontal axis in the aquarium, the animal endeavors unceasingly to creep upward as often as the plate is turned through an angle of $90^{\circ}$. We are not dealing in this case with a compensatory motion brought about by centrifugal force; while the plate is being turned the animal remains quiet, and not until some fifteen or twenty minutes later does it begin to move upward.

Nor do we deal in this case with the effect of daylight entering the aquarium from above. If the animals are kept

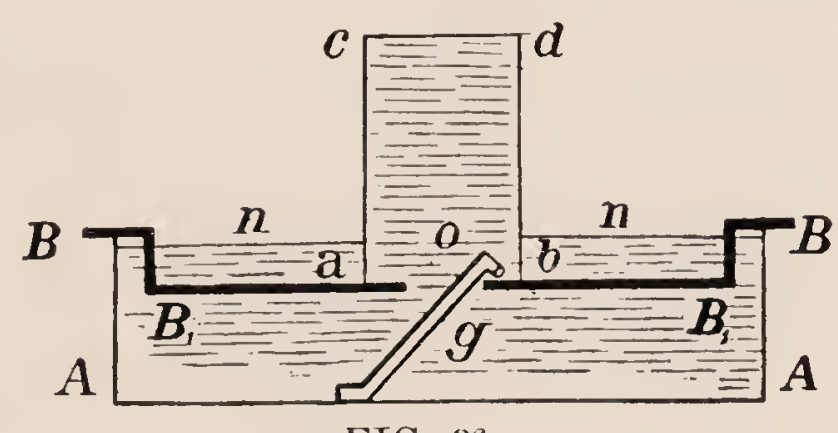

FIG. 36 in an aquarium into which light is allowed to enter by suitable means only from below and without, the animals continue to creep up the vertical surfaces, the direction in which they move not being influenced in any way by the light.

One might think that the need of oxygen determines the movements of Cucumaria toward the surface of the water, but it can be shown that this also is not the case. If a large beaker, from which the air has been removed by filling it with water, is placed in an aquarium upside down - that is to say, with the bottom of the beaker directed upward - the Cucumariæ nevertheless continue to creep up to the bottom of the beaker. They do this even when the experiment is made as shown in Fig. 36. A bridge $B B$ is suspended in the aquarium $A A$ so that its horizontal part $B_{1} B_{1}$ is below the surface of the water $n$ in the aquarium. In the bridge is a small circular hole o, over which the inverted beaker $a b c d$, in which the air has been displaced by water, is placed. Fresh water is supplied through o under slight pressure by means of a suitably curved glass tube $g$. The Cucumarix nevertheless leave the neighborhood $o$, collecting either on the bottom of the beaker $c d$ or upon its vertical sides near $c d$. 
Yet the hydrostatic pressure does not compel the animals to move upward to the surface of the water, for when the animals are put into a flat dish containing only $1-2 \mathrm{~cm}$. of water, so that the animals are just under the surface of the water, they also move to the vertical wall of the vessel and attach themselves to it, even though they are no nearer the surface of the water in this position than anywhere else in the aquarium. The nature of the conditions of the experiment shuts out the possibility of rheotropism and hydrotropism.

Experiments with the centrifugal machine yielded no results, as the animals do not move at all during the rotation of the machine. The one condition which compels the animals to seek vertical surfaces and crawl upward is gravitation. I imagine that the way in which gravity compels the animals to move upward is similar to that which compels insects, such as the butterflies which have just left their pupa case, to crawl upward. Immediately after hatching, the wings of the butterfly are not yet unfolded, and the animal runs about in a restless way until it reaches a vertical surface upon which it creeps and remains, with its head directed upward, for a relatively long time, until the unfolding of the wings or other conditions cause the animal to become restless again. In cockroaches this dependence of rest and unrest upon gravitation is still more apparent. They remain perfectly quiet only when the weight of their bodies exerts a pull upon their legs, but not when the weight of their bodies presses upon their legs. Cucumaria cucumis seems to behave in a similar way. The direction of the pull, however, influences in addition the direction of the progressive movements upward. Yet it is possible that these reactions in Cucumariæ and insects are dependent upon specific organs, as in the case of vertebrates.

This relation of Cucumariæ to gravitation compels the 
animals to live in the upper regions of the sea. If a larva were driven into deep water, it would be compelled to crawl upward without ceasing, through its negative geotropism, until it had reached the surface again, or until death brought an end to these efforts.

2. Other marine animals, which we also know to live only in the upper regions of the sea behave as does Cucumaria cucumis. Actinia mesembryanthemum of the Bay of Naples belongs to this class. If I remember correctly, however, I have never observed this behavior in the Actinian of the East Sea, which has the same name, but differs somewhat in its characteristics, from that found in the Bay of Naples. But certain starfish, such as Asterina gibbosa, are also negatively geotropic. All the experiments which I have made on Cucumaria are successful also in Asterina gibbosa, with this difference, however, that when the exceedingly voracious Asterina has reached the highest point on a vertical surface and finds no nourishment there, it does not remain in this position permanently, as does Cucumaria, but begins to move or drop down after two days or even less time. Asterina gibbosa also lives only at the surface of the sea.

Positive heliotropism acts, of course, in a way similar to negative geotropism. Asterina tenuispina lives near the surface of the sea, as does Asterina gibbosa. It is, however, not geotropic, but positively heliotropic. I laid a large number of the two species together in a heap in an aquarium into which light fell almost horizontally from one side only. Within a short time the two kinds of animals were clearly separated; the tenuispinæ crawled along the bottom toward the source of light; the gibbosæe distributed themselves in all directions over the bottom and crawled upward upon the vertical walls without the direction of their movements being influenced by the light. In the ocean, in which only those rays of light which enter vertically are of importance for the spatial 
orientation of the animals, positive heliotropism must compel Asterina tenuispina to move to the surface of the water in the same way as negative geotropism does Asterina gibbosa. Since Asterina tenuispina is always positively heliotropic (and does not alter its heliotropic sense as do the nauplii of Balanus), periodic depth-migrations do not occur in it as they do in the nauplii of Balanus because of the change in the heliotropic sense of the latter.

3. Preyer briefly mentions in his exhaustive work on the movements of starfish "the tendency of these animals to crawl upward" which he noticed in his observations on the climbing movements of starfish. Preyer writes as follows:

The great tendency of starfish to move upward cannot be dependent upon lack of oxygen, lack of food, or changes in the temperature, or currents in the water, nor to a desire for light, for the upward movements occur also where these conditions are not at work. Some property of the bottom, or of that particular spot on the bottom upon which the animal rests, and which has become unfit for the attachment of the animal or its prolonged residence, probably compels the animal to climb upward. Yet parasites which I often found in the ambulacral feet of Luidia may also cause them to move upward, in so far as these stimuli may appear to the animal as originating from the horizontal bottom. ${ }^{1}$

The first sentence in this generalization is incorrect, as light is indeed the factor which compels Asterina tenuispina to crawl upward. It is shown by the following experiment that the nature of the bottom upon which the animal rests does not compel it to move upward. If Asterina gibbosa is placed in a cubical box having glass sides, the animals leave the horizontal basal wall and climb up the vertical sides. If the box is turned through an angle of $90^{\circ}$ about a horizontal axis, the animal leaves the wall, which has now become basal, and climbs up to, and remains upon, the wall which it left before when it lay horizontally. The character of the wall was p. 96.

1 W. Preyer, Mittheilungen aus der zoologischen Station zu Neapel, Vol. VII, 
therefore not the cause of the movement of the starfish. Finally, when Preyer believes that parasites compel the animals to move upward, it is impossible to see why the parasites do not compel the animals which are fastened upon the vertical wall to leave it, as the stimuli caused by the parasites must now seem to the animals to originate from the vertical wall. Yet in reality Asterina gibbosa (and much more Cucumaria cucumis) remains upon the highest point of the vertical wall. I believe it much more rational to take into consideration the effect of gravitation, which acts in a vertical direction, in explaining the upward movements of certain starfish.

\section{THE GEOTROPISM OF HIGHER ANIMALS AND ITS DEPENDENCE UPON THE INNER EAR}

The higher animals are also compelled, within certain limits, to assume a definite orientation toward the center of the earth. It is easily seen in many fishes that they orient themselves toward the center of the earth, both while swimming and while at rest, and that they direct their bellies, but never their backs, downward. If we try to compel such a fish to lie upon its back, it endeavors and succeeds, as soon as liberated, in reassuming its usual orientation. It is not physically impossible that such a fish should swim with its back downward, and only physiological factors are present which compel the fish to direct its belly toward the center of the earth. Even we are compelled to assume a certain position with reference to the center of the earth; we discover it when we bend our heads so that the top of the head is downward. The force which compels us to assume a definite orientation toward the vertical is naturally not very great, yet it has a definite intensity, and it requires an external stimulus of a certain intensity, or a definite effort of the will, to act against this force in order to overcome it. 
There is a second, better observed and more accurately studied, effect of gravitation upon higher animals. This has to do with the axes of the eyes, which are also compelled to assume a definite position with reference to the horizontal. If the head of a fish is forcibly brought into a different position from that which it occupies normally with reference to the center of the earth, the eyes tend to reassume, either entirely or in part, their old orientation. These lasting changes in the position of the eyeballs with reference to the axes of the head which accompany a permanent change in the position of the head are also present, as is well known, in human beings. They can in this case, as in the case of animals, be compensated through appositely working optical conditions or internal stimuli, but a definite force exists in this case also to compel the axes of the eyes to assume their proper angle with the horizontal.

Light has nothing to do with these phenomena; they occur also, as is well known, in the dark, and in persons totally blind.

We deal rather in these cases, as we know, with the activities of a definite organ, namely, the inner ear. Schrader found that frogs from which the inner ear has been removed upon both sides no longer endeavor to regain their position when laid upon their backs, ${ }^{1}$ and Breuer has corroborated this observation. ${ }^{2}$ According to a paper by Sewall, ${ }^{3}$ to which I do not, however, have access, and with which I am acquainted only from the abstract which Breuer gives of it, the compensatory movements of the eyes in sharks and skates were seriously affected by such lesions of the labyrinth as led to decided and permanent disturbances in their movements. These experimental facts, indicative of the dependence of geotropic orientation upon the ear, are not numerous

1 Schrader, Pflügers Archiv, Vol. XLI.

2 Breuer, Pflügers Archiv, Vol. XLVIII, p. 237.

3 Sewall, Journal of Physiology, Vol. IV. 
compared with those on the dependence of movements upon the ear. And so it happens that Aubert and Delage ${ }^{1}$ acknowledge the latter relation, but do not, as it seems to me, acknowledge the former. I myself do not doubt the belief of Breuer and Mach ${ }^{2}$ that the organs associated with the permanent changes in the position of the eyeballs, in movements of the head, lie in the inner ear; and I think it probable, in further accord with these authors, that these organs are more especially the otoliths. I therefore add the following results, obtained in a large series of experiments on the inner ear of the shark (Scyllium-canicula), principally to show that my ideas of the importance of the inner ear in the geotropism of the higher animals rest upon personal observations. $^{3}$

I. If the otoliths are removed (either with a pair of forceps or a small sharp spoon) from a shark (Scylliumcanicula) upon one side, say the left, the following changes occur in the animal in its orientation toward the center of the earth:

1. The animal no longer swims, as does the normal, so that its medium plane is vertical, but it has a tendency to turn the left or operated side downward at an angle of from 20 to $50^{\circ}$, or even more, with the horizontal.

2. The same change in the orientation can be noticed when the animal is lying quietly upon the bottom of the aquarium. It then frequently lies resting upon the left lateral fins, while the right fin often does not touch the bottom of the dish.

3. When the animal is in the normal primary position,

1 AUBERT, Physiologische Studien über die Orientirung (Tübingen, 1888).

2 MACI, Grundlinien der Lehre von den Bewegungsempfindungen (Leipzig, 1875), p. 110 .

3 In an addendum to his paper on the Functions of the Central Nervous System, etc. (Braunschweig, 1888), STEINER describes his experiments on "the seni-circular canals of sharks." This author has overlooked the fact that it is the function of the inner ear to call forth compensatory motions and positions, and so has failed to make observations on the geotropic functions of the ear. (See MACH and BreUEr.) 
its eyes are rotated more or less about the long axis of the body toward the left, so that the left eye looks downward, while the right eye looks upward. The (persistent) geotropic movements of the eyeballs upon changing the orientation of the animal toward the center of the earth still occur, with this modification, however, that when the whole animal is turned about its longitudinal axis the amount of the compensatory movement of the eyes is added algebraically to that caused by the lesion of the ear.

4. Only a slight change is noticeable, usually, in the position in which the pectoral fins are kept. The left lateral fin is turned toward the back while the right is turned toward the abdomen.

II. If the otoliths of such an animal are removed from the other side also, all the phenomena described above disappear. Instead, however, now the following abnormalities appear:

1. The animal is no longer compelled to turn its ventral side toward the center of the earth. If one carefully attempts to lay it upon its back the animal does not resist, and when prevented from falling over, it remains permanently upon its back. When left to itself, the animal is often found lying upon its back, or swimming in this position, even when it is entirely well and vigorous.

2. The permanent alterations in the position of the eyeballs, when the orientation of the animal toward the center of the earth is changed, are lacking.

III. If the antrum is opened and the same injuries are inflicted as are necessary to remove the otoliths-without, however, injuring these or the nerves of the antrum-the phenomena described under I and II do not appear. Nor is this the case when large pieces are removed from the semicircular canals without injuring the ampullæ.

IV. If the left auditory nerve of the animal is cut, the 
phenomena described under I are observed, only in a greater degree. If both auditory nerves are cut the phenomena described under II set in.

$V$. If the otoliths are removed from one side and the auditory nerve is cut upon the other, the animal behaves as those described under II; with this difference, however, that the eyes of the animal are turned about the longitudinal axis of the animal toward that side where the auditory nerve is cut.

Also, as regards the so-called forced movements - which are not considered in this paper-the animal behaves as if it had been operated upon only on one ear, namely, that whose auditory nerve is cut.

VI. A shark whose head has been cut off, and which, as is well known, still swims, is no longer forced to assume a definite orientation toward the center of the earth. When laid upon its back it no longer attempts to reassume its accustomed position with its ventral side downward.

The disturbances in the geotropic orientation which follow the cutting of the auditory nerve have thus far continued for a month.

These observations, it seems to me, prove that the geotropic phenomena observed in the shark are dependent upon the inner ear, and support the assumption of Breuer and Mach that they are called forth by the ototiths.

But we can determine how the inner ear forces an animal to maintain a certain position with reference to the horizontal as little as the botanists can explain how geotropic curvatures are called forth in plants. We can only say, in harmony with Goltz, ${ }^{1}$ or Mach and Breuer, that the animal comes to rest only under a certain arrangement of strain and pressure in the peripheral ends of both auditory nerves. This arrangement exists in the shark when the animal turns

1 Goltz, Pflügers Archiv, Vol. III. 
its ventral side toward the center of the earth, and keeps its longitudinal axis almost horizontal; but in every other position of the animal the altered distribution of strain and pressure upon the ends of the auditory nerves forces the animal again to assume its customary angle with the horizontal. This force is maximal when the animal lies on its back. If one of the auditory nerves is cut the animal is compelled to assume an oblique position in which the injured side is directed downward more than usual; if both auditory nerves are divided, no force exists to compel the animal to assume any definite geotropic position. ${ }^{1}$

I will give a single example to illustrate what may be the further biological significance of geotropism. The reader is acquainted with the marked difference in the pigmentation of the ventral and dorsal sides of many animals, especially that of the fishes. "This difference is to a large extent independent of light and is determined by conditions which accompany development. In part, however, it is directly dependent upon light; the back, which is permanently directed toward the source of light, must become richer in pigment than the ventral side. Cunningham has, indeed, been able to show recently that flatfish develop pigment upon their lower surfaces in an abnormal way when these are illuminated by the help of mirrors, as strongly as their upper surfaces. ${ }^{2}$

1 I no longer believe that the direction of the waves of sound has any effect, which I considered possible in my preliminary paper on geotropism.

2 J. T. CunnINGHAM, Zoologische Anzeiger, Vol. XIV (1891). 


\section{VI \\ ORGANIZATION AND GROWTH ${ }^{1}$}

I. THE DEPENDENCE OF ORGANIZATION IN ANTENNULARIA ANTENNINA UPON THE ORIENTATION OF THE ANIMAL TOWARD THE CENTER OF THE EARTH

1. THE difference between physiological morphology and the purely descriptive morphology lies in this, that the former endeavors to control the formation of organs. Its aim is, therefore, primarily a technical one. Since almost everything is still to be done in this direction, at present those cases will be especially welcome in which the means of control of the morphogenetic processes are very simple and yet perfect. I have found that in Antennularia antennina, a Hydroid, it is possible to control the morphogenetic process with the aid of the position given it with reference to the center of the earth. By this means we can not only at will cause another organ to grow in the place of an amputated one, but we can compel an uninjured existing organ to stop growing in its old form and form an entirely different organ.

2. In Antennularia antennina (Fig. 37) a straight unbranched stem $S S$, about $1-2 \mathrm{~mm}$. in diameter and often more than $20 \mathrm{~cm}$. long, arises from a mass of roots $W$. Delicate lateral branches of limited growth and directed slightly upward spring from the stem in regular order. Upon the upper surface of these are polyps and nematophores, which are indicated only by points in the drawing. In order to save room, only the lower third of the stem has been drawn.

I have previously pointed out that Antennularians show a decided geotropism; that is to say, the growing parts of in 1891.

1 Würzburg: Georg Hertz, 1891. Although dated 1892, this pamphlet appeared 
these animals are compelled to assume a definite orientation toward the center of the earth. ${ }^{1} \quad$ If the growing stem of an Antennularian is put int 3 any other than a vertical position.

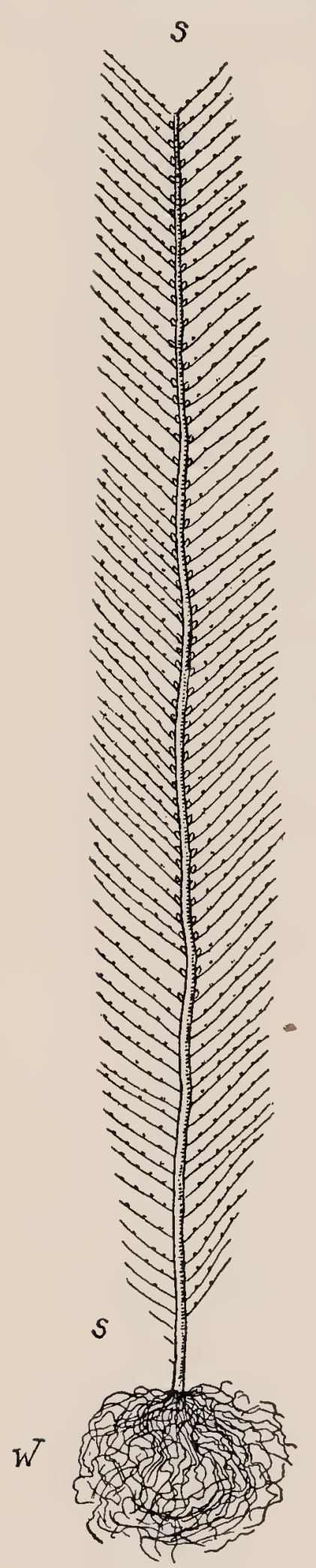

FIG. 37 the growing tips bend until the stem again stands vertically, after which it grows straight upward. The root, upon the other hand, grows vertically downward, but not so directly as the stem. The stem is negatively, the root positively, geotropic. The stem $a b$ (Fig. 38) originally stood vertically. I tilted it so that it formed an angle $c b a$ with the vertical; the newly formed piece $b c$ grew vertically upward after this change in position. I then put the stem back into its old position, after which growth continued in a vertical direction cd. Fig. 39 also shows an upward bending of the stem. Roots growing downward are shown in $W$ (Figs. 40 and 41). While the tip of the growing stem does not weary of growing vertically upward whenever its position toward the horizon is slightly altered, the root is much more sensitive. It does not bear

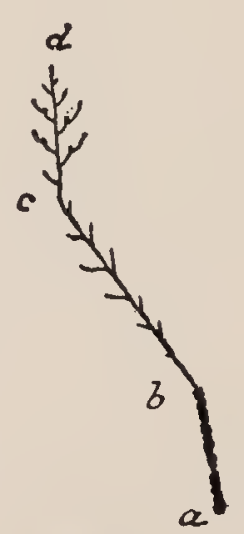

FIG. 38

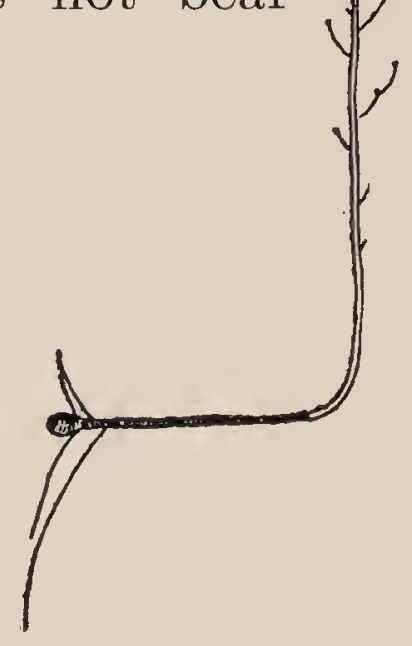

FIG. 39

changes in position very well, and I have been able to demonstrate positive geotropism only in newly grown

1 Part I, p. 176. 
roots. Light has no demon- a orientation of Antennularia. one side only, the animals and the same occurs also in uli, however, affect the orien- strable effect upon the Even when lighted from continue to grow upward; the dark. ${ }^{1} \quad$ Contact stimtation. When the roots

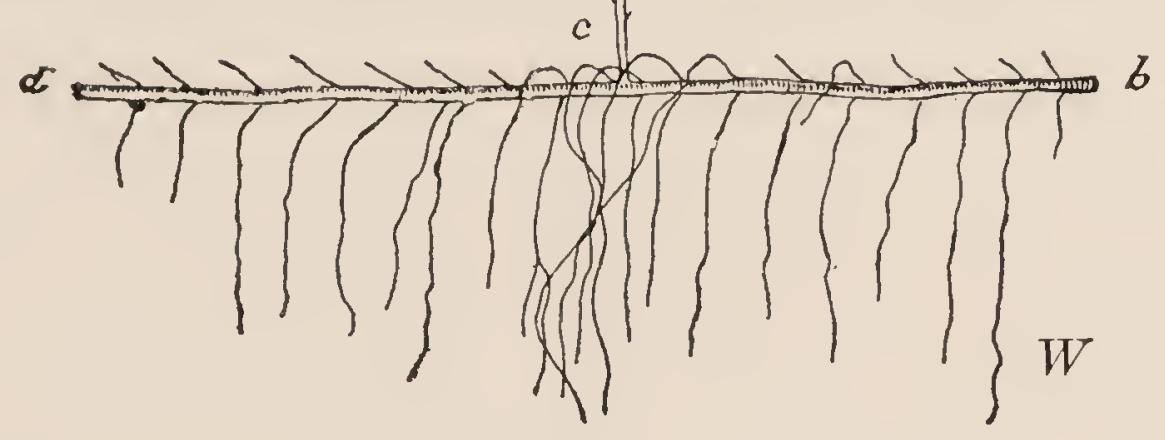

FIG. 40

are brought in contact with a solid body, they attach themselves to it and grow over its surface. The stems and branches do not possess this form of irritability.

3. With these preliminary remarks, I shall show how regeneration and heteromorphosis depend upon the orientation of the $\alpha$

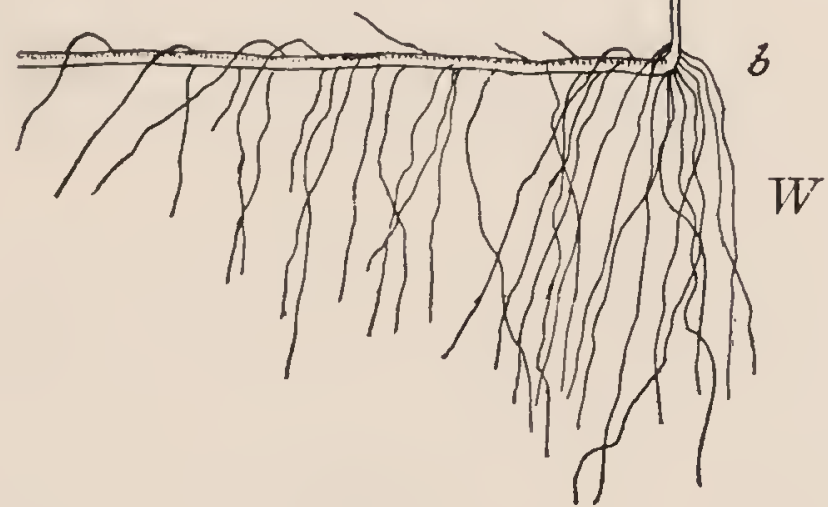

FIG. 41

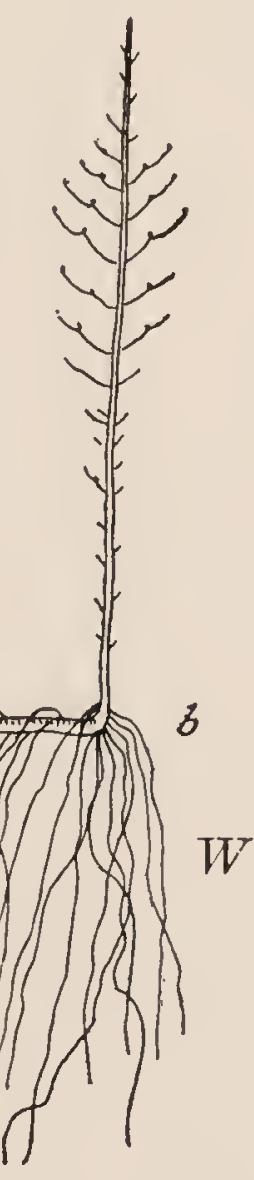

$W^{\top}$

stem with reference to the horizon.

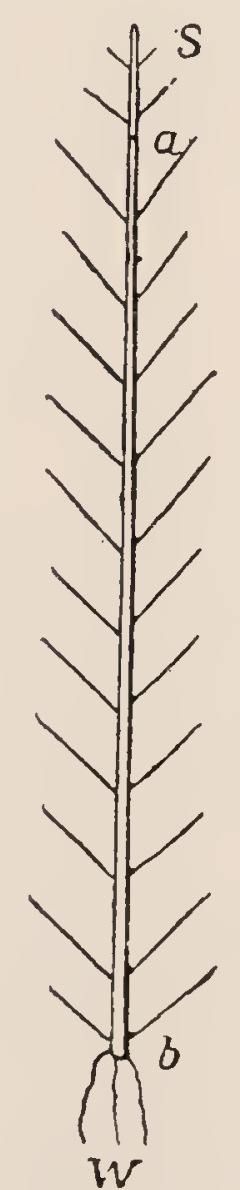

FTG. 42

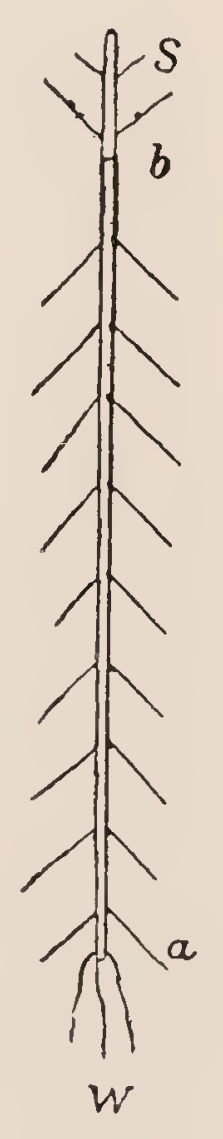

FIG. 43 a piece cut from the stem of an Antennularian; $a$ is the

1 The specimen grew only slightly in the dark. This may, however, have happened only by chance. 
apical and $b$ the basal end (root). $a b$ is suspended vertically in the aquarium so that both cut ends are surrounded by water; $a$ is directed upward, $b$ downward. Complete regeneration occurs, a new stem $S$ being formed at $a$, while one or more roots $W$ are formed at $b$; the former grows

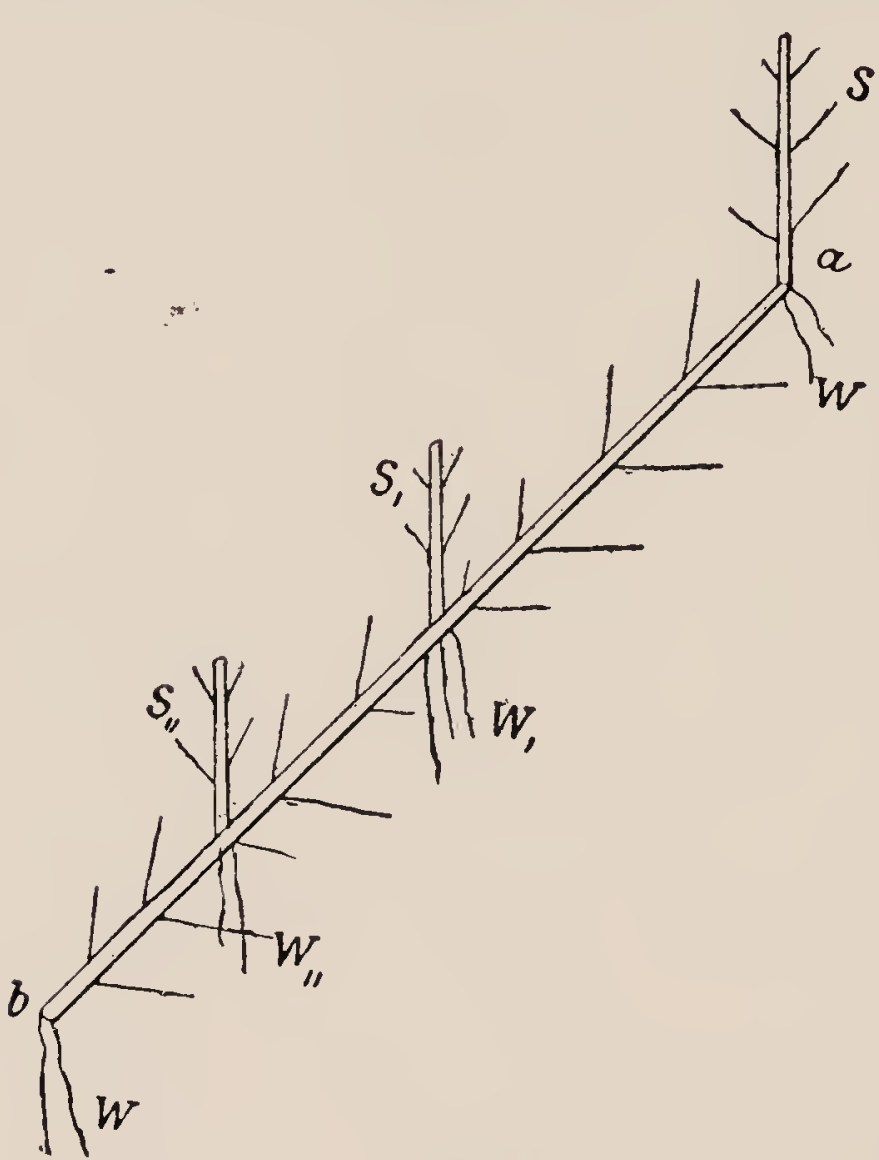

FIG. 44 upward, the latter downward.

We repeat the same experiment with another piece of stem, only this time the basal end $b$ is directed upward, while the apical end $a$ is directed downward. A stem $S$ is formed at the basal end which is directed upward, while one or more entirely normal and growing roots are formed at the apical end which is directed downward (Fig. 43). Deli-

cate lateral branches spring from the new stem which are directed slightly upward and carry polyps upon their upper surfaces. The new branch is an image of the old. In this way a heteromorphosis is readily brought about in Antennularia.

4. If a piece of the stem of Antennularia is suspended, not vertically, but obliquely, in the aquarium (Fig. 44), a stem $S$ which grows vertically upward arises from the upper cut end; from the lower end $b$ spring roots which grow vertically downward; it matters not whether $b$ is the apical or the basal cut end. Thus far this experiment shows nothing new. But branches $S_{\mathrm{i}}$ and $S_{\mathrm{ii}}$, and roots $\mathrm{W}_{\mathrm{i}}$ and $\mathrm{W}_{\mathrm{ii}}$, may arise from any other element of a stem put obliquely. The 
essential point is again this, that the place of the new growth is determined by the orientation of the stem toward the center of the earth, inasmuch as stems arise only from the upper surface, the roots only from the lower surface, of the element. That a new stem may arise from the upper surface of any

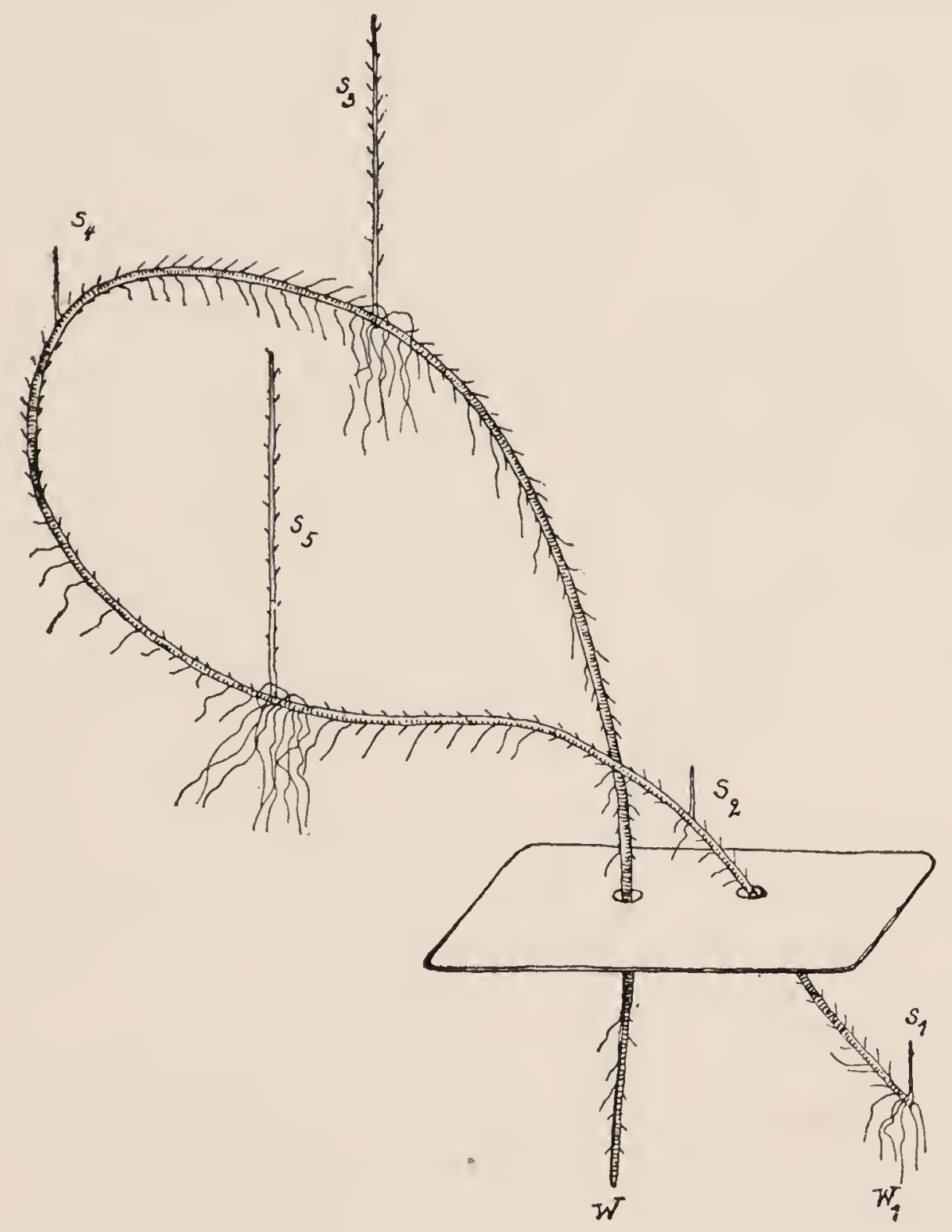

FIG. 45

element of a stem which is put obliquely is evidenced by the fact that such stems arise at times from the upper surface of the lowest elements of the animal. Such a case is shown in Fig. 45. The stem of an Antennularian was bent so that the two ends were directed obliquely downward. New stems, $S_{1}, S_{2}, S_{3}, S_{4}, S_{5}$, have formed upon the upper surfaces of different elements of the old stem. $S_{1}$ has grown from 
the upper surface of the lowest element. That stems are formed upon the upper, while roots are formed upon the lower, surfaces of the stem, when it occupies any other than a vertical position, is best shown when stems of an Antennularian are suspended horizontally in the aquarium

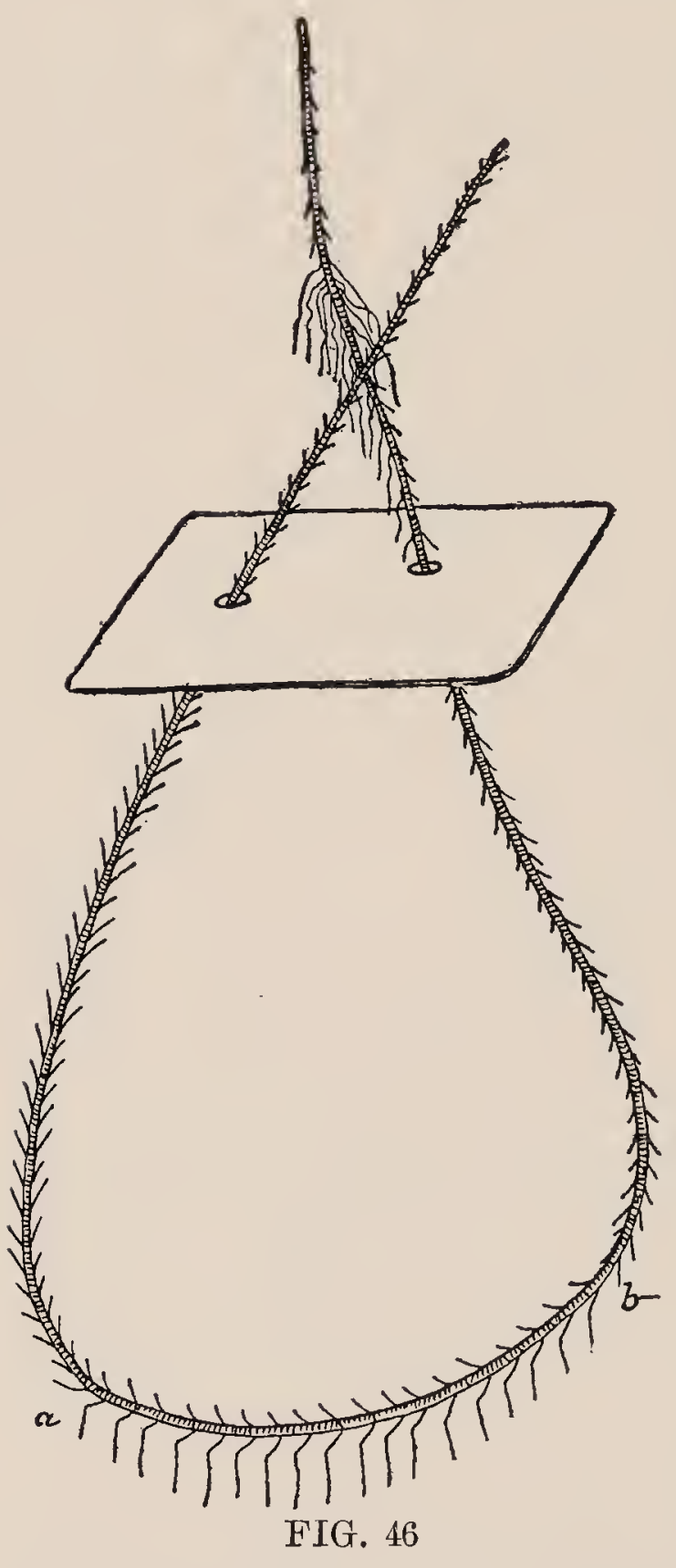
so as to be surrounded on all sides by water. If the experiment is continued for a long time, other phenomena occur, especially root formations, a discussion of which we shall give later. If this fact is borne in mind, Fig. 40 may serve to illustrate such an instance. In Fig. 41 is shown how a new stem arises from the basal cut end and from the upper surface of a stem lying horizontally.

II. CONTINUATION OF THESE EXPERIMENTS AND HETEROMORPHOSIS IN UNINJURED ORGANS

1. It is not only possible by the aid of gravity to cause the growth of another organ in place of one that has been lost, but we can also cause an uninjured organ to grow into a typical organ of a different kind by making use of the same force.

If a piece is cut from the stem of an Antennularian, and this is laid horizontally into the aquarium so as to be surrounded by water, the tiny lateral branches situated on the under side of the stem begin to grow after some time- 
about one or two weeks. But they do not grow in the old direction obliquely toward the tip of the stem, but instead vertically downward. Nothing of this sort happens to the branches situated upon the upper surface. The newly formed organs which grow vertically downward look exactly like

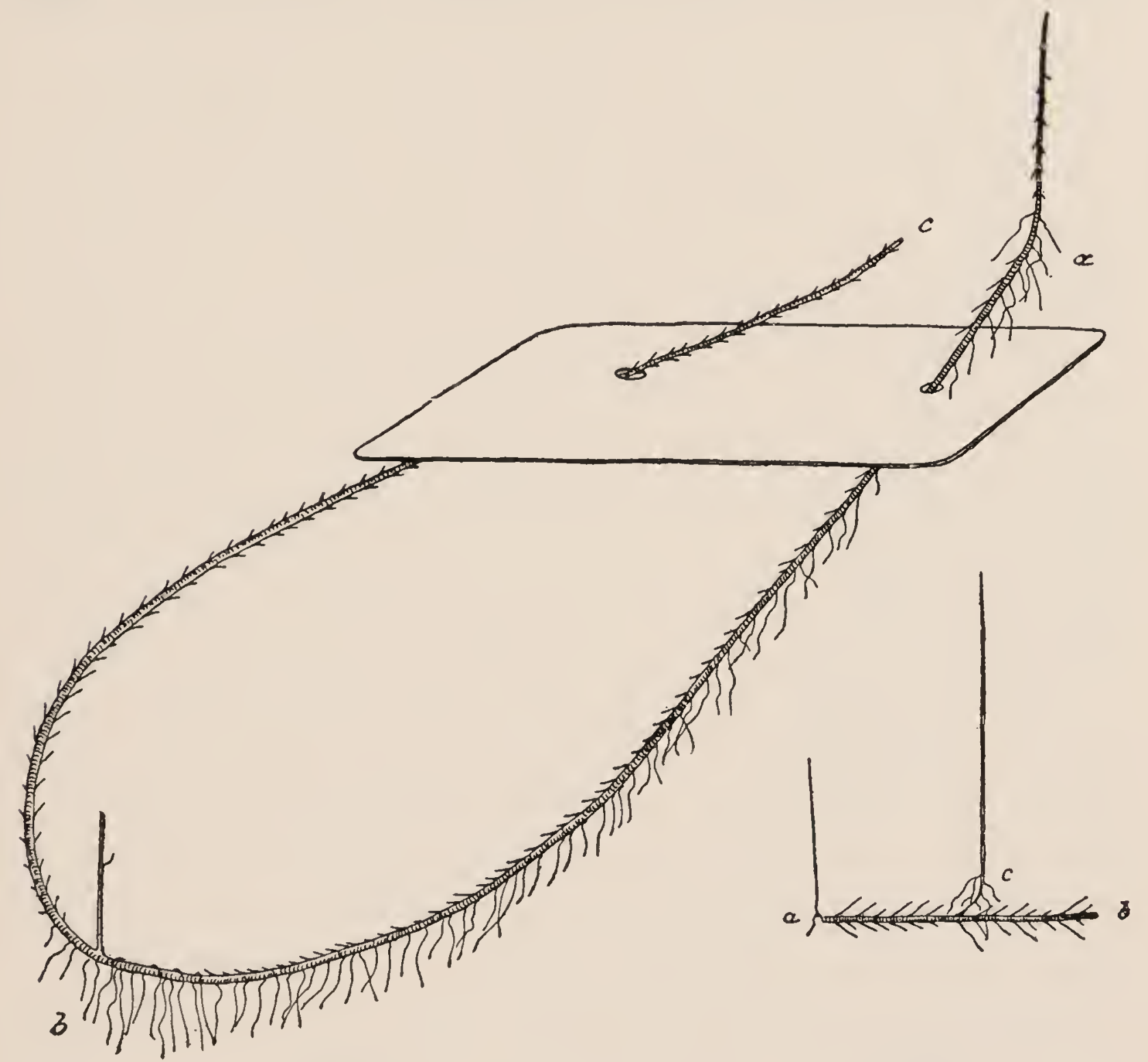

FIG. 47

FIG. 48

roots, and that they are indeed such can be proved physiologically. If they are brought in contact with a solid body, they attach themselves to it and grow over its surface. Besides being positively geotropic, they are also positively stereotropic - a form of irritability possessed only by the roots, but not by the branches, of Antennularia. Such an Antennularian stem is shown in Fig. 40, in which all the branches of the lower surface have been transformed into 
roots. Several of the branches on the upper surface in the neighborhood of the growing stem $c d$ have also become roots, but this is an exception which we shall discuss later.

2. I cut some long pieces from the stem of an Antennularian, bent them, and hung them into the aquarium, as shown in Figs. 45, 46, 47. Both ends were oriented in the same way toward the center of the earth. After some time (Fig. 46) the branches of the under surface of the nearly horizontal piece $a b$ began to grow as roots. Some time later the same occurred in all those branches, the tips of which were directed downward. In Fig. 47 is shown a second specimen of the same series of experiments, but drawn six week later. All the branches from $a$ to $b$, the tips of which were directed downward, have grown into roots. By carefully studying Fig. 45 this phenomenon can again be observed; only I must call attention to the fact that this drawing was made very shortly after the beginning of the experiment, and that the formation of roots had consequently not progressed very far.

Macroscopically the course of the heteromorphosis of a branch is as follows: The tip of the branch situated upon the lower side of the stem dies, and the polyps and nematophores disappear. The new root then sprouts from the free distal end of the branch, without any operative injury whatsoever having been inflicted upon the branch.

3. While I always succeeded under the conditions described above, in causing the lateral branches to grow into roots, I succeeded only once in making them grow into a stem. This is shown in Fig. 48. A short piece $a b$ of an Antennularian stem was laid horizontally in the water. After some time the tip of one of the lateral branches $c$ began to grow vertically upward. No new lateral branches were formed upon it. A similar negatively geotropic new growth arose from the aboral cut end at $a$.

4. The heteromorphoses possible through changes in the 
orientation of Antennularia toward the center of the earth are not exhausted by what has been said in the foregoing. It is possible to cause the growing tip of the stem to cease its growth and develop into a root. This is done by inverting the tip. I cut long pieces from the stems of Antennulariæ and hung them vertically in the aquarium, but in an inverted position. A rapidly growing stem was formed at the upper-that is to say, the basal-end. When it had attained a certain height, I turned the whole animal about a horizontal axis through an angle of $180^{\circ}$. Not in a single instance did the tips of the stems bend upward-as the negatively geotropic parts of plants usually do under the same conditions-but they ceased to grow and one or more roots formed at their tips.

Fig. 49 shows a stem at the end of such an experiment; $a b$ is the piece of stem upon which the experiment was started. It was at first fixed

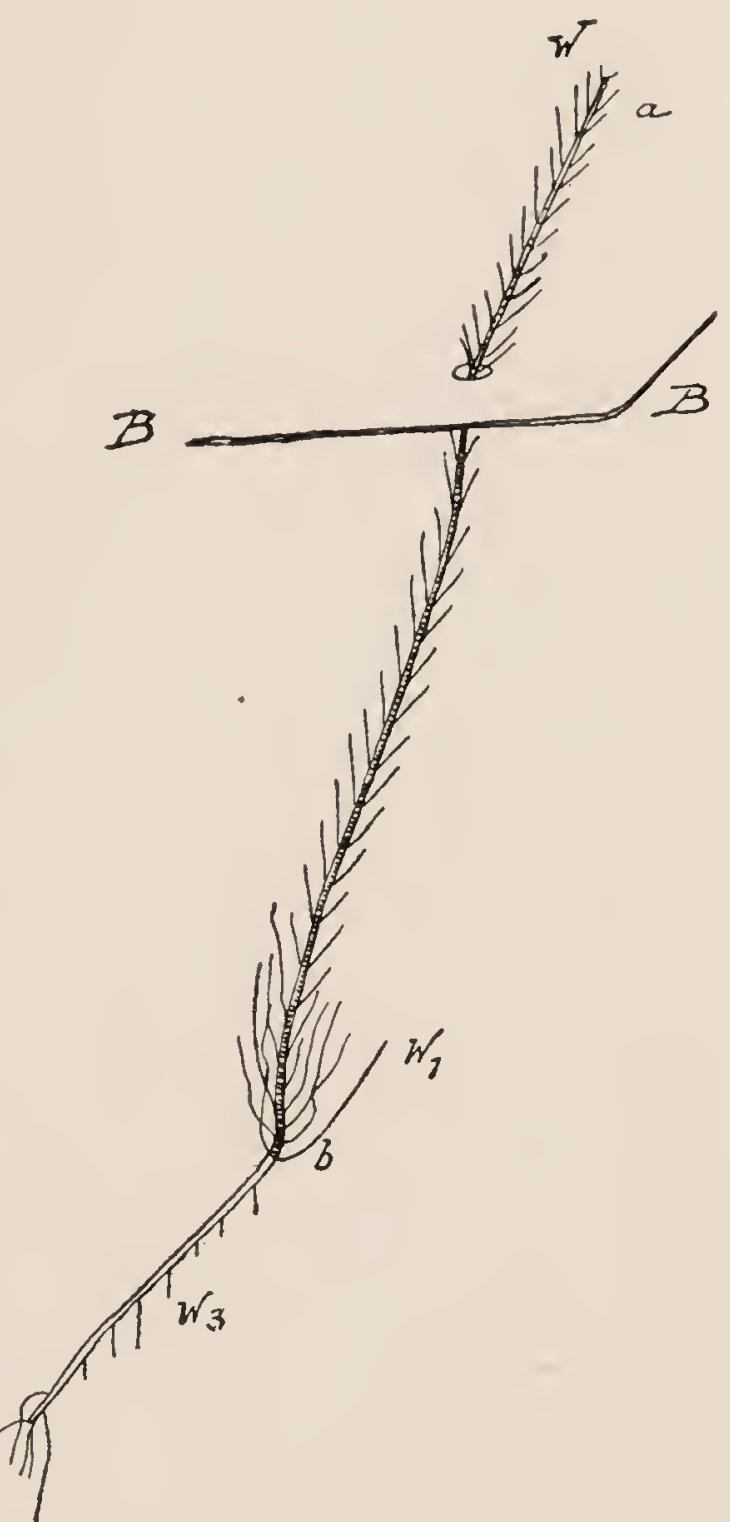

FIG. 49 in an inverted position, so that the basal end $b$ was directed upward, and the apical end downward. The new stem $b c$ sprang from the basal extremity, grew vertically upward, and gave rise to lateral branches which were directed slightly upward and carried polyps upon their upper surfaces. The roots $W_{1}$ formed at $b$, at the base of the new stem, as was usually the case when the old stem did not stand entirely vertical. 


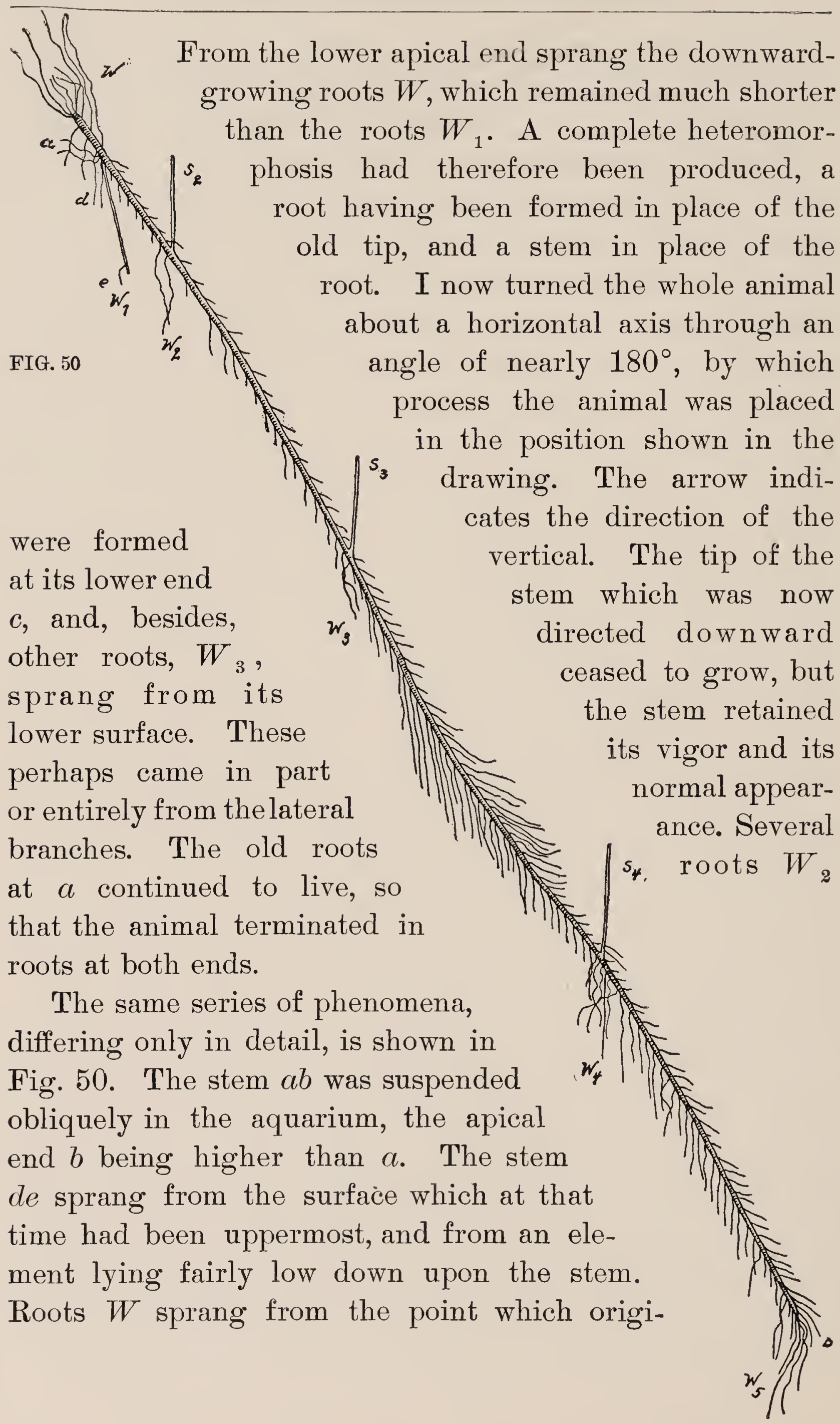


nally lay opposite de, and from the lower cut end $a$ of the stem. When the new stem had attained the length de, I turned the whole animal about its axis, so that $b$ and $e$ were directed downward, and the specimen was in the position indicated in the drawing. The stem de ceased to grow, and a delicate root $W_{1}$, growing downward, was formed at $e$. The drawing was made soon after the root began to grow. Besides being formed at $e$, roots $W_{5}$ also grew from the apical cut end $b$ which was directed downward; the three stems $S_{2}, S_{3}, S_{4}$, were formed upon the upper surface of the stem with their corresponding roots $W_{2}, W_{3}, W_{4}$.

5 . In all these experiments one fact - which is, however, not a specific property of Antennularia - constantly repeats itself: new growths arise much more easily and numerously from the basal than from the apical end. I have observed the same fact in Actinia mesembryanthemum, and A. von Heider mentions it in connection with Cladocora. ${ }^{1}$

In general, too, the formation of roots in Antennularia is dependent upon the formation of a stem. If a stem is formed at any point, roots also form after some time, at first upon the under surface of the old stem, then upon the upper surface in the immediate neighborhood of the new stem, and finally at the lower end of the new stem itself (Fig. 40). It is self-evident how such phenomena complicate the influence of orientation upon the place where new organs are formed.

6. I cannot say precisely how the position of the new organs formed in Antennularia is determined by the orientation of the animal toward the center of the earth. Naturally the effect of gravitation cannot be different in animate from what it is in inanimate nature, and there would be no reason for being disappointed should it be found that the apparently mysterious effect of gravitation upon organization in Anten-

1A. vow HeIder, Wiener Sitzungsberichte, Vol. LCCCIV, Part I (1881), p. 664. 
nularia consists, for example, in simple hydrostatic effects. It would also be entirely useless to ask whether gravity is "the cause" of organization in Antennularia; naturally it is only one of the conditions determining organization. Under ordinary conditions, however, it is of paramount importance. ${ }^{1}$

I add these remarks since it seems to me that the work of Pflüger on the effect of gravitation upon the division of cells is misunderstood in some of its points, and its value to the further development of physiological morphology is not appreciated as it should be.

7. I shall in conclusion describe an experiment which I found in the botanical literature on the transformation of organs in plants through external forces. The reader may see from it that plants and animals are not essentially different in regard to the physiology of organization.

I choose as an illustration Noll's ${ }^{2}$ experiments on Bryopsis, which perhaps represent the most successful experiments ever made on the control of organization in plants through external forces. According to Noll, the

so-called "leaf-barbs" (leaves) of Bryopsis mucosa arise near the tip of the upright stem. They are hollow tubes which spring from the main stem in two rows at an angle of about $45^{\circ}$. The roots arise from the lower part of the plant and grow away from the light into the ground, where they become attached to the particles of earth much as do the root hairs of the higher plants. The stems and leaves do not show this reaction to contact stimuli.

The morphological differentiation of the organs seems to be much less marked in Bryopsis than in Antennularia, for, according to Noll, "by examination of a single organ doubt can easily arise as to whether the fragment originates from a root, a stem, or a leaf tubule." In a normal Antennu-

1 In his Grundriss der Naturlehre, MACH defines cause and effect as follows: "Die auffallendste Bedingung der eingetretenen Veränderung pflegt man die Ursache, die Veränderung selbst die Wirkung zu nennen." 1888).

2 F. NoLL, Arbeiten des botanischen Instituts in Würzburg, Vol. III (Leipzig, 
laria it is an easy matter to diagnose the organ from which a fragment has originated. If such a Bryopsis is laid horizontally upon the ground, "the growing stem becomes erect at its tip, the leaf tubules again grow obliquely upward at their usual angle, and the root tubules grow downward into the dark earth." Noll then tried "whether a root could be produced from the tip of the stem, and a new stem from the root tubules." He suspended plants from which the roots had been cut in glass tubes in an inverted position, that is, with the tip downward. When the experiment was brought to a close after some time,

the tip in a number of plants had greatly increased in length, but instead of growing upward it had grown downward into the sand, was bent, and intimately connected with the grains of sand; in short, it had been transformed into a typical root tubule. Even the few leaves which had been formed had also grown into roots. Another series of these specimens did not show this transformation; in these the tips turned upward at an acute angle and returned in their old direction; they remained stems.

In regard to the latter point the behavior of Antennularia is essentially different. When the tip is directed downward, its growth as a stem ceases immediately; never under these circumstances does it bend so as to grow in its old upward direction.

Further growth can now take place only in so far as the tip continues to grow as a root. In Noll's experiments a tubule which grew upward arose at the upper basal end, and in some cases showed a beginning barbule formation. According to Noll, light is the essential factor in the control of the morphogenesis.

III. THE ARTIFICIAL PRODUCTION OF A VARIETY OF ANTENNULARIA ANTENNINA

The main stem of Antennularia antennina is unbranched. In the hundreds of specimens that passed through my hands 
in Naples I did not find a single exception to this rule. When a piece was cut from the stem of Antennularia antennina and a new stem grew out of it, either through regeneration or through heteromorphosis, the newly formed stem had, under ordinary circumstances, all the characteristics of
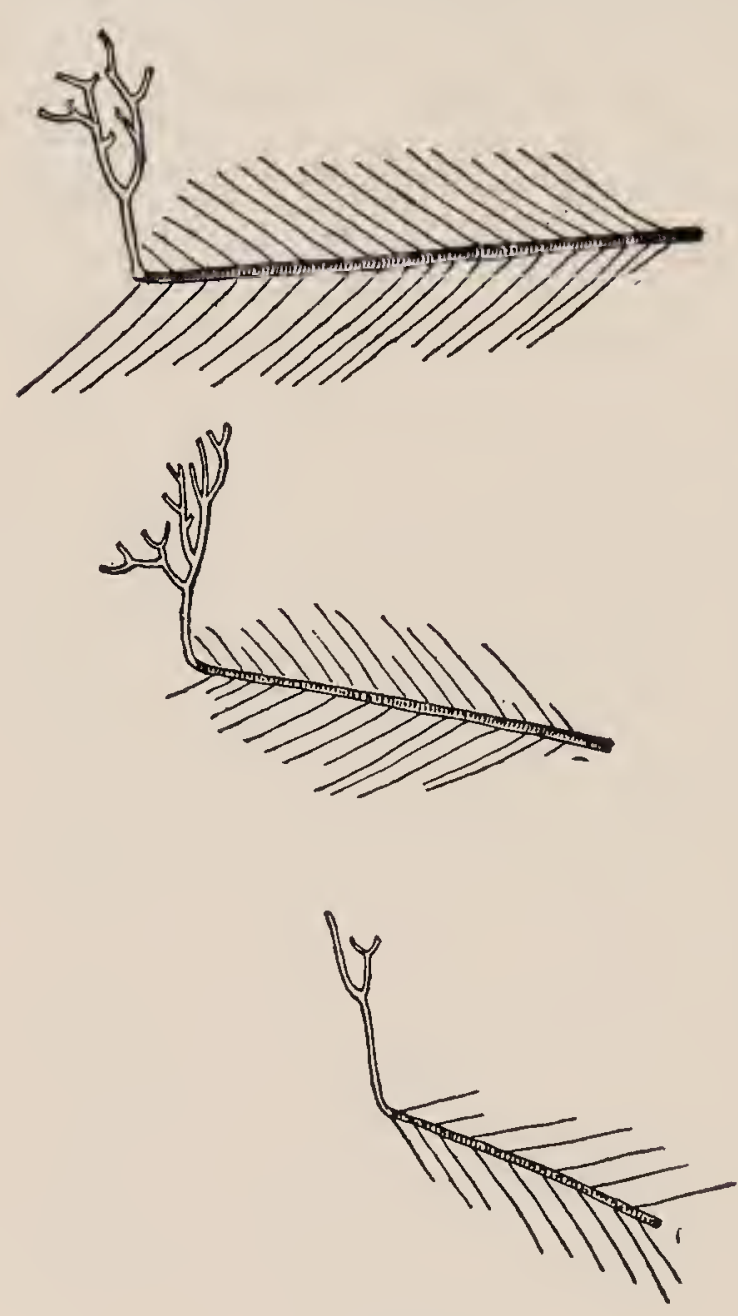

FIG. 51 the old-it grew upward without branching.

Under certain external conditions, however, an entirely different kind of stem was regenerated. At first a short stem grew straight and vertically upward, but from this soon sprang a new branch having the diameter of the old. Since every branch one after another gave rise to other new lateral branches, which after a time no longer grew perfectly vertically upward, forms of Antennularia with many ramifications originated. Faithful representations of three such forms are given in Fig. 51. They all arose from stems lying absolutely horizontal, or nearly so, two at the apical end and one at the basal end. No branches were formed.

I have, moreover, made experiments to decide whether pieces of the old stems which under abnormal conditions gave rise to variations would produce unbranched or branched antennina stems when brought back to normal conditions. Under these conditions they formed only unbranched antennina stems.

Under the abnormal conditions, however, not all the stems branched; this happened only in part of the specimens. 
I shall have to continue the experiments before I can discuss the conditions which determine the formation of these abnormalities.

\section{ON THE INTERNAL CAUSES OF ORGANIZATION IN}

\section{TUBULARIA MESEMBRYANTHEMUM}

1. In most animals at a cut edge with a definite orientation only one kind of organ originates. If the tail of a lizard or a salamander is amputated, only a tail and not a head is regenerated in its place, and a lobster develops a new pincer in place of the one lost, and never anything else. ${ }^{1}$ Nor can it be said that only the higher animals show this inflexibility in organization. As shown by all the experiments which have been made upon it for a hundred and fifty years, Hydra behaves thus; even in Infusoria Nussbaum found such a relation to exist between the new growth and the orientation of the wound. ${ }^{2}$ In plant physiology the instances in which complete control of organization through external forces is possible are very scarce. In the majority of these experiments, also, it seems that internal, and at present unknown, factors essentially determine the position of the organs. If we wish to control these internal conditions in animals, we must try to obtain further information concerning them through experiments. In order to give the reader a clearer idea of the rôle of these internal causes in animal morphogenesis I will remind him of the experiments upon Cerianthus membranaceus, given in the first volume of my Physiological Morphology. ${ }^{3} \quad$ The oral pole $a$ of Cerianthus differs markedly morphologically from the aboral pole $b$ (Fig. 52). To be brief, I will mention only one differencethat tentacles arise from the oral pole. If a rectangular piece

1 This is no longer correct. Herbst has sbown that in Crustaceans in the place of an eye a new eye or a new tentacle can be produced at desire. [1903]

2 M. Nussbaum, Archiv für mikroskopische Anatomie, Vols. XXVI and XXIX.

3 See Part I, p. 115. 
cdef is cut from the body of an Actinian, tentacles are formed upon only one of the four cut edges, namely, upon the oral surface ef. From this and similar experimentswhich may be read in the original-it follows that the place where the new tentacles are formed in a fragment of Cerianthus is determined by the orientation which

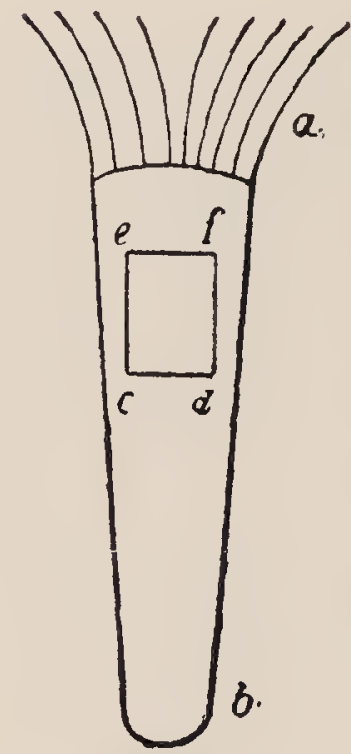

FIG. 52

the fragment had in the intact animal; just as in every fragment of a broken magnet the position of the poles is determined by the orientation of the piece in the unbroken magnet. From this analogy - which, however, goes no further - the term "polarity" has recently been applied to the organization of such animals. According to this idea, Cerianthus would be a typical "polarized animal."

Now, Cerianthus is not a satisfactory subject for investigation of the causes of "polarity ;" Tubularia mesembryanthemum, a Hydroid, adapts itself much better to these experiments. ${ }^{1}$ Tubularia ends in a root at its aboral end, and in a polyp at its oral end. If a piece is cut from the stem, and both cut ends are surrounded by water, a heteromorphosis results, as I have shown in a former paper. A polyp arises from both cut ends, and a bioral animal is obtained. Yet the formation of polyps is different at the two ends in one particular: it is always more rapid at the oral than at the aboral pole. In so far as this fact renders it possible to determine which was the oral and which the aboral end in the uninjured stem, it can still be considered as an expression of polarity. The following experiments deal with the internal conditions which determine the difference in time between the formation of the polyps at the oral and at the aboral ends in Tubularia.

The starting-point of these experiments is J. Sachs's 1 See Part I, p. 115. 
theory of organization." Sachs assumes that "with differences in the forms of organs are comnected differences in the substances composing them," and that "from principles which hold for all sciences we must assume that the differences must be derived causally from the differences in chemical constitution" (p. 425). "We shall have to assume the existence of just as many specific formative substances as there are different forms of organs in the plant." The specific morphogenetic substances are affected through external conditions, especially gravity and light, "so that in certain cases the spatial arrangement of the different organs is determined thereby." The monstrous formation of an organ at the place where normally another organ should exist - as in the case of heteromorphoses - Sachs attributes to an absence of the specific substances necessary for the formation of the normal organ at this place, and the presence instead of the specific formative substances of another organ (p. 464). Sachs also explains why any regeneration whatsoever of roots or stems occurs in plants deprived of them. "Why is it that the simple removal of a piece calls forth a regeneration of organs at places where this never would have occurred without some disturbing influences such as the removal of the piece?" (p. 470). The answer is as follows :

I assume that as long as a green-leafed plant with an upright stem is nourished and growing, the specific formative substances for the root flow from the assimilating leaves to the system of roots at the lower end of the stem, while the substances forming the stem flow in a similar manner upward toward the growing points of the stem and the branches. If a piece is cut out of the stem or the root, the cut surface in itself offers a barrier to the further movement. The specific formative substances contained in it will, in consequence, collect in the neighborhood of the two cut surfaces. Those substances leading to the formation of roots will collect at what

$1 \mathrm{~J}$. Sacrs, Arbeiten des botanischen Instituts in Würzburg, Vol. II (Leipzig 1882), pp. 452, 689 . 
has thus far been the lower end of the piece, while those substances leading to the formation of stems will collect at the upper end. Since their flow is stopped here, which would have been impossible in the uninjured plant, they give rise to the roots and stems at the corresponding ends. In a piece of leaf capable of regeneration both kinds of formative substances will be moving simultaneously toward the basal end to flow toward the stem; stopped

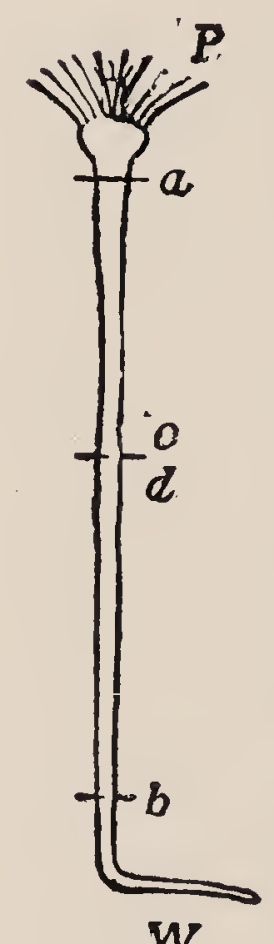
by the cut surface, they collect there to form stems and roots simultaneously. (P, 470.)

Finally, "the different formative substances are produced only in limited quantities" (p. 468).

Corresponding to this theory we assume that there are present in the stems of a Tubularian specific substances for the formation of polyps which gather at both cut ends and thus bring about the formation of polyps. These substances must, however, ceteris paribus, collect more quickly, or in sufficient amounts, sooner at the oral than at the aboral cut end; and the cause of

$w$ this difference is to be determined by experiment.

FIG. 53

3. It might at first be thought that the specific substances necessary for the formation of polyps exist from the beginning in greater amount at the oral end of a Tubularian stem than at the aboral, and that a polyp is, in consequence, formed more quickly at the oral than at the aboral end. To ascertain whether this be true I amputated the root and the polyp of Tubularians and cut the remaining stem $a b$ (Fig. 53) in half by a transverse incision between $c$ and $d$. All four cut ends were surrounded by water. If now the substance necessary for the formation of polyps were present in larger amounts in the oral half $a c$ than in the aboral piece $b d$, the former should form polyps sooner than the latter. Yet polyps were formed at about the same time upon both pieces, only the oral cut ends $a$ and $d$ developed polyps much sooner than $c$ and $b$. I have already 
described this experiment in the first volume of my Physiologicul Morphology. During my last stay in Naples $\bar{i}$ repeated it, and found that in one case the polyps at $a$ were formed about twenty-four hours earlier than at $d$. But this difference is not great enough to explain the difference in time in all cases between the formation of polyps at the oral and at the aboral end, which not infrequently amounts to one or more weeks.

4. Another possibility is conceivable. The substances necessary for the formation of polyps are formed in the stems of the Tubularia, and move in both directions, but the movement occurs first in the direction from root to oral pole (upward), and more easily than in the reverse direction. If this assumption were correct, then when we divide the stem $a b$ as above, the polyp at the oral cut end $d$ of the aboral piece $b d$ ought to be formed distinctly earlier than at the aboral end $c$ of the oral piece $a c$, because the formative substance of the polyp has to move in the first case in the direction from root to polyp (upward); in the second, in the reverse direction.

I picked out eight healthy specimens of Tubularia from a colony, amputated root and polyp, and divided the remaining pieces $a b$ by a transverse incision between $c$ and $d$. The pieces $a c$ were fixed in an inverted position, the pieces $b d$ in an upright position in narrow glass tubes standing vertically, so that only the ends $c$ and $d$ were surrounded by water and could form polyps. After three days two polyps were formed on the $d$ ends; upon the following day all the $d$ ends carried polyps. On this day the formation of polyps only just began on the $c$ ends in two of the specimens; not until four days later was regeneration complete in all the specimens.

In a second experiment I used nine Tubularian stems. After three days all the $d$ ends had polyps, while regeneraation had only begun in two $c$ ends; only three days later 
had regeneration begun in all of these specimens. In other experiments, also, the same results were obtained. That the orientation of the Tubularian stem toward the center of gravity has no effect I have mentioned in the preceding paper iv; and I have corroborated this finding by careful control experiments. I suggested in paper iv that regeneration perhaps occurs more rapidly at the oral pole because a distinct difference in the diameter of the lumen usually exists between the two extremities. The experiments cited above show that this suggestion is incorrect; there is no difference in diameter at a transverse section, and yet regeneration always occurs much earlier at the one cut end than at the other.

5. If the Tubularian stems used in the experiment are very turgescent, and highly pigmented, the difference in time between the formation of the oral and the aboral polyps may be very slight, and amount to only a few days. Very often one finds pale Tubulariæ which form polyps within the usual time at the oral pole, in which, however, the polyps at the aboral pole are formed only after one or more weeks. I made the following experiment upon such animals: I fixed eight animals (I) taken from one colony vertically, in the sand, but with their oral ends downward, in order to prevent the formation of polyps at these ends. At the same time I suspended seven other animals (II) of the same colony also vertically and in an inverted position in the aquarium, but so that both ends were surrounded by water. These animals had to form polyps at both cut ends. All animals were kept in the same aquarium, and were therefore exposed to the same temperature. After three days the first few new polyps were formed at the oral pole of those Tubularians (II) of which both ends were surrounded by water. "On the next day all of these had formed polyps at the oral cut end. The same day, however, polyps were formed in five of the animals 
of the other group (I) - at the aboral end; and on the day following, regeneration was complete in all of the animals of this group. In Group II, on the other hand, in which the animals had formed polyps at the oral pole, regeneration did not set in at the aboral cut end until five days later; and not until six days after this was regeneration complete. The formation of polyps at the aboral pole was therefore complete eleven days sooner in the animals of Group I, in which the formation of polyps was prevented at the oral pole, than in the animals of Group II, in which polyps were formed at the oral pole. By preventing the formation of polyps at the oral pole, the formation of polyps at the aboral can therefore be accelerated. I have repeated this experiment some ten times, but always with the same result; usually the delay in the formation of polyps at the aboral end caused by allowing polyps to develop at the oral end was even greater than that here given. This simple experiment, which can easily be repeated, and, according to my experience, with certainty, may be thus explained in the light of Sachs's theory. The specific formative substances for the polyps are present only in limited amounts, these being sufficient for the formation of only one polyp in the Tubularian stem at the moment that it is cut. When both poles are subject to the same external conditions, these substances reach the oral cut end first. If, however, the formation of polyps is rendered impossible at this end, they wander to the other end. ${ }^{1}$ If the formation of polyps is not prevented at the oral end, a polyp can form at the aboral end only after a sufficient amount of the formative substance for the polyps has been formed anew.

6. The question now arises as to whether in the stem of Tubularia such a migration of substance toward the cut end can, indeed, be observed to precede the regeneration of a

1 It is possible that in some cases of compensatory hypertrophy, for example in the testicles, specific formative substances for the organs play a similar role. 
polyp. If a Tubularian stem is examined immediately after the polyp has been cut off, separate red pigment granules are found distributed quite regularly throughout the stem. On the following day a denser aggregation of these granules is observed in the vicinity of the cut ends, and after two or three days they are often so numerous that the cut end seems saturated with red. Soon thereafter the polyp is formed into which the red granules are collected. This occurs also in the formation of a polyp at the aboral end. I will not, of course, assert that these pigment granules are the formative substances of the polyps in the sense of Sachs's hypothesis. It is rather to be assumed that, since pigment granules have no active motion, movements probably occur in the protoplasm of the Tubularian stem, at first in the direction from the aboral end to the oral, and later, when the oral polyp has been formed, in the reverse direction. It might also be that in some cases in which external stimuli have an influence upon organization these stimuli bring about or modify such protoplasmic streaming. The movement of protoplasm in plants under the influence of external stimuli has been observed directly by Wortmann. ${ }^{1}$ The migration of the pigment granules toward the cut ends actually observed, and the connection between this migration and the formation of polyps, probably indicate that one is justified in believing that organization in Tubularia-and also other animals - is associated with the migration of formative substances.

7. For the time being we may, therefore, believe that a heteromorphosis can be brought about whenever the specific formative substances can wander in different directions in the animal body; while in the case of animals possessing "polarity" this migration is possible in only one direction. When heteromorphosis occurs in Tubularia, but the oral

1 Wortmann, Botanische Zeitung (1887). 
polyp is formed sooner than the aboral, we should have to assume that the substances necessary for the formation of new polyps move in both directions in the Tubularian stem, but this movement occurs more rapidly, or earlier, in the direction from root to polyp, than in the opposite direction.

8. I shall in conclusion mention the fact that under one condition the polyp may occasionally be formed sooner at the aboral pole than at the oral, even when both are subject to the same external conditions. This can happen when the formation of polyps is retarded, or made almost impossible, through external conditions. According to my former observations, this can also happen when the pieces of Tubularian stem used for the experiment are very short.

\section{IRRITABILITY AND ORGANIZATION IN TUBULARIA}

We saw that the physical condition which determines the orientation of Antennularia in space has an effect also upon the position of its organs; the same relation also exists between irritability and organization in Tubularians. Tubularia mesembryanthemum is neither heliotropic nor geotropic; light and gravity also have no effect upon the origin of new organs in Tubulariu. On the other hand I have found that the animal is highly stereotropic; that is, the orientation of its organs is very clearly affected by contact with the surface of solid bodies. The root of Tubularia is positively stereotropic, that is, when brought in contact with the surface of a solid body it attaches itself to it and grows over the surface. Possibly through the slight friction a substance is secreted which causes the root to stick. The polyp, however, is negatively stereotropic. When brought in contact with a solid body, it turns away from it until it is again surrounded by water, after which it grows in the direction determined by the orientation of the polyp. The same stimulus which determines the orientation of Tubularia in 
space is also of importance for its organization. For it is possible to control organization in Tubularia by contact stimuli to such an extent that by their help we can prescribe in a large measure whether a root or a polyp shall grow from the aboral cut end of a Tubularian. If the aboral end of a piece of Tubularian stem is surrounded by water, so that it does not rub against the surface of solid bodies, almost without exception a polyp is formed upon it instead of a root; heteromorphosis occurs instead of regeneration. If, however, the same end is brought into permanent contact with the surface of a solid body-if, for example, the stem is laid horizontally upon the bottom of the vessel--a root is often formed. But to accomplish this it is necessary to keep the dish quiet, and care must be taken that the same side of the stem remains in contact with the solid body; otherwise it does not attach itself. If the stem does not attach itself, a polyp is soon formed, just as a polyp is formed at the tip of a Tubularian root which has been freed from the bottom of the vessel, as soon as it is surrounded by water. Of course, it is in every case necessary that the general conditions of growth of the animal, which we shall discuss later, be fulfilled. I remember that only once in hundreds of individual experiments did I see a root grow without contact with a solid body. ${ }^{1}$ But a factor came into play here which still brings this case under the general grouping. I had cut a section from the stem of a Tubularian, and had laid it horizontally upon the bottom of a glass vessel. A root sprang from the aboral end which attached itself to the bottom of the vessel. Simultaneously with this root arose a second, which curved upward somewhat, and which continued to grow as a root without contact with a solid body. The exception was probably

1 In recent experiments on Tubularia crocea I have frequently observed the formation of roots at the aboral end, even if the latter was not in contact with a solid body. [1903] 
only apparent. It may be that I had to do with a bifurcation of a root which had been produced through contact stimuli, of which the one branch continued to grow without contact. But, as before, I did not succeed in causing the growth of a root at the oral pole in even a single instance; it may be that this is impossible in Tubularia by any of the means which I have employed thus far. In the light of my previous experiments on Antennularia and Aglaophenia, I need not emphasize that this is only a special characteristic of Tubularians.

If a root is formed at one extremity of a Tubularian, and this has grown vigorously for some time, its tip not infrequently becomes faintly red, and this coloration deepens rapidly; in such cases a polyp forms at the tip of the root, which then immediately bends away from the substratum upon which the root lias grown and grows almost perpendicularly upward. We then have a colony of two polyps which are attached to the base upon which they grow by a common root. The difference between such an animal and the heteromorphic bioral animal lies in the fact that the latter lacks an inherited organ, the root. In its place a polyp has been formed.

\section{EXPERIMENTS ON ORGANIZATION IN CIONA INTESTINALIS}

1. The animals in which I have thus far been able to produce heteromorphoses stand low in the animal scale; we have dealt only with Hydrozoa. It might be thought that these "colony-forming" animals are an exception to the general rule, and that only in them a movement of formative substances can take place in both directions in the sense of Sachs's theory. Such a view is supported by the fact that "polarity" is very pronounced in a series of Actiniæ, and that, in spite of my efforts, I have not succeeded in bringing about heteromorphoses in them. The fact that a solitary 
Hydroid, Hydra, should conduct itself, so far as known, as a strictly "polarized" animal, might render it still more probable that heteromorphosis is simply a characteristic of "colony-forming" Hydrozoa. To settle this question I made

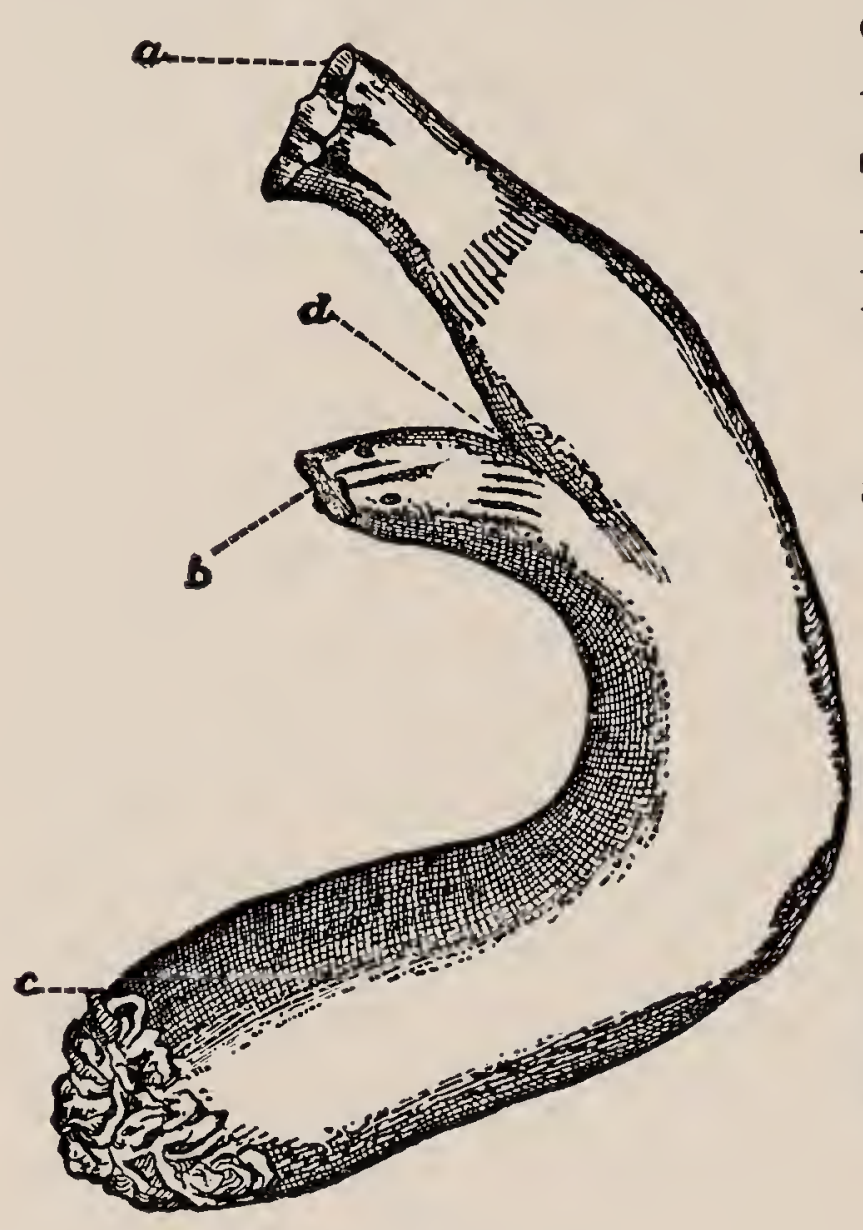

FIG. 54 experiments upon Ciona intestinalis, a solitary Ascidian. The Ascidians, as is well known, stand near the vertebrates in the animal scale.

In Fig. 54 is shown in almost natural size the exter-

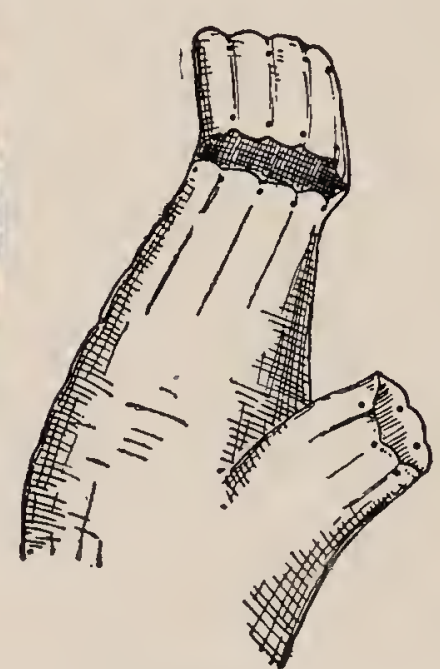

FIG. $5 \tilde{j}$

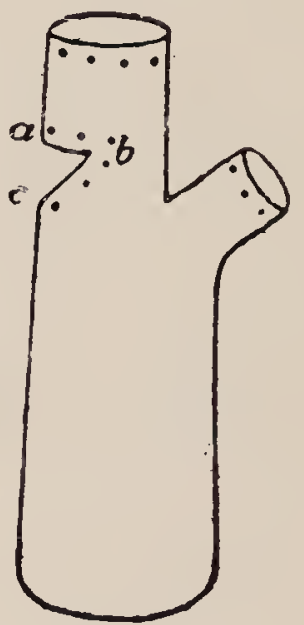

FIG. 56

nal form of a Ciona intestinalis. $a$ is the oral opening, $b$ the excretory opening, $c$ the foot, which, like the foot of the Hydrozoa, attaches the animal to the surface of solid bodies. At the outer edge of both tubes are situated the ocellæ, which are just visible to the naked eye (Fig. 55). The following experiments deal with the formation of these ocellæ. If the animal behaved like a purely "polarized animal," such as Cerianthus, then, when a lateral incision cba (Fig. 56) is made into it, ocellæ should be formed only along the lower cut edge $b c$, just as the tentacles are formed only along the lower cut edge of Cerianthus. But the opposite occurs in Ciona. After a short time ocellæ (indicated by points in the drawing) are formed along both edges of the wound ( $a b$ and 
the regeneration of the central nervous system. Scarcely another animal is so well adapted to the study of this problem as Ciona. The brain of Ciona consists of a snow-white ganglion, somewhat larger than the head of a pin, situated in the angle $d$ near the surface between the two tubes $a$ and $b$ (Fig. 54). The mantle of Ciona is very transparent, so that the ganglion can be removed with certainty without seriously injuring the other organs of the animal. The only difficulty encountered in the experiments is that the animal contracts when touched, and that the ganglion is then no longer visible. I overcame this difficulty in the following way: The root of a Ciona which had been kept in the aquarium was carefully detached from its base. The animal was then quickly removed from the water and laid upon a dark glass plate. As long as the animal was not touched, it remained relaxed. I then brought the tip of a pair of scissors behind the ganglion, and by a rapid cut severed the connection between the ganglion and the pedal part of the body. Without removing the scissors, I seized the ganglion with forceps, drew it out, and removed it by a second snip of the scissors.

The Ascidian usually bears the extirpation of the ganglion well, and after the operation behaves in a way not expected from our general conceptions of animal physiology.

2. The first phenomenon worthy of note is that after extirpation of the central nervous system the reflexes persist. Since Ciona is a sessile animal, the reactions of the uninjured animal to external stimuli are limited to simple contraction and stretching out of the body. This contraction is brought about by a highly developed muscular system. If the aquarium is slightly shaken, the animal contracts quickly, to relax only when everything is again at rest. Such a contraction of the whole animal also often follows when it is carefully touched with the point of a needle. If 
the tip of one of the tubes is touched, this only may contract. It is generally believed that when this occurs the external stimulus which brings about a change in the sensory nerve endings is conducted to the central organ, here to be transformed into a motor impulse which causes the muscles to contract. These ideas harmonize with the facts. But it is also generally believed that without the central nervous system the reflexes are no longer possible. This idea, as the following experiments will show, is not true in Ciona intestinalis. When the ganglion has been removed from a Ciona, it at first remains fully contracted. After some time, however, in favorable cases, as early as the next day the animal again relaxes. If a drop of water is allowed to fall into the basin of water, the entire Ciona contracts rapidly, just as an animal whose central nervous system is intact.

If, therefore, a normal Ciona and one without the central nervous system are kept in the same vessel, both have the same reflex irritability qualitatively. It is nevertheless possible to differentiate clearly between the reaction of the normal and the brainless Ascidian. In the latter the threshold of stimulation is much higher than in the former. To determine this I utilized a procedure which I have employed in a series of comparative experiments on the irritability of the lower animals, and which might be used with advantage in human beings. I allow a drop of water to fall from a pipette upon the organ to be stimulated, which lies a certain distance (varying according to circumstances) beneath the surface of the water. If the same pipette is always used, the weight of the drop is nearly always the same, so that the height which the drop must fall just to bring about a reaction is a convenient measure of the threshold of stimulation. In what follows I shall give the height of the fall of a drop of water which just sufficed to bring about a contraction of the entire Ciona. The normal and the brainless animals 
were kept in the same vessel, and at the same distance below the surface of the water. Under $a$ are given the height of the fall in normal, under $b$ in brainless, animals. The figures are given only to show how constant is the difference in the threshold of stimulation between the two animals; the absolute height of the threshold of stimulation cannot be determined from them.

\begin{tabular}{rl}
\multicolumn{1}{c}{$a$} & \multicolumn{1}{c}{$b$} \\
$8 \mathrm{~mm}$. & $65 \mathrm{~mm}$. \\
4 & 75 \\
10 & 80 \\
& 80
\end{tabular}

In two other animals I obtained the following values.

\begin{tabular}{ll}
$a$ & \multicolumn{1}{c}{$b$} \\
$6 \mathrm{~mm}$. & $22 \mathrm{~mm}$. \\
8 & 20
\end{tabular}

The temperature was $13^{\circ} \mathrm{C}$.

This difference in irritability may be due to the fact that the wave produced by the drop of water stimulates the motor nerves or muscles directly, and that the threshold of stimulation is higher for these than for the sensory nerve endings.

3. It can be shown by a different experiment that another path for the conduction of stimuli must exist in the brainless animal than in the uninjured, where stimuli no doubt travel over the nerves for the most part. If an incision is carefully made in the tube of a Ciona whose ganglion has been removed, not only the injured portion, but the entire animal, contracts, just as when the same stimulus is applied to an uninjured animal. This also may occur when one point of the oral edge is carefully touched with a needle. I imagine that in a brainless Ciona the motor nerves or muscles lying nearest the point of incision are stimulated mechanically, that these contract, and that the jarring or pulling 
associated with this contraction stimulates the neighboring nerves or muscles, thus causing the latter to contract. In this way a conduction of stimuli is brought about without the presence of a central nervous system, the effect of which is the same as when a central nervous system is present. There is so little difference between the latent period of stimulation and the time the body remains contracted after the stimulation, in the normal and in the brainless animal, that it cannot be determined by mere observation unaided by special apparatus.

What occurs here in the entire animal happens in a limited portion of an earthworm when it is cut across transversely and the two pieces are sewed together. As Benedikt Friedländer has shown, both pieces are still able to perform co-ordinated movements of locomotion. ${ }^{1} \quad$ I have repeated the experiments of Friedländer upon leeches, and have observed the same series of phenomena in them.

Two years ago I made some observations upon a marine Planarian, Thysanozoon brocchii, which are similar in certain respects to those upon Ciona, into a discussion of which, however, I cannot enter here. ${ }^{2}$

4. Since I had received the impression that Ciona is heliotropic, I tried to see whether a Ciona robbed of its nervous system would also react to light by heliotropic bendings. The object of my experiments was thwarted by an unwished for, but perhaps interesting, result. In the course of four weeks all the animals (which had not died) had regenerated a new brain. I repeated the experiment, because I did not believe my first results. But I can no longer doubt

1 Benedikt Friedländer, Biologisches Centralbl., Vol. VIII (1889).

2 The Darwinians would probably call the reaction of a Ciona intestinalis to contact the consequence of a "protective instinct." These "protective instincts," so far as I can see, are said to consist in this, that the animal has by natural selection acquired, in the course of the customary million years, certain cerebral contrivances which are now inherited from one generation to another. But in the case of Ciona these hereditary "instincts" cannot well be located in any special portion of the brain, for they continue to exist after the removal of this organ. 
their correctness. The growth of the new ganglion is fairly rapid. Instead of one large ganglion, several small ones were formed. For these reasons I have not followed further the question as to whether Ciona is at all heliotropic. Dr. P. Mingazzini has also observed the regeneration of the brain of Ciona after its removal; he suggests that the new ganglion is formed from the ectoderm, as in the embryo.

\section{THE RELATION BETWEEN REGENERATION AND THE} CONCENTRATION OF THE SEA-WATER IN TUBULARIA

1. Now that we have seen that it is possible to alter the inherited form of an animal by substituting one organ for another, we shall pass to experiments on the general physiological factors which underlie regeneration and growth in animals. These experiments, as the preceding ones, had to be made with the simplest experimental accessories, and in addition they were not completed when I was compelled to leave Naples. Since they can be continued only at the seashore, I shall here give only those experiments which have been carried to a certain degree of completion.

If the polyp of a Tubularian is amputated, a new polyp regenerates. What general physiological conditions must be fulfilled in order that this may occur? Since the stem begins to grow as soon as the polyp is formed, the second question arises: What conditions influence growth? The following experiments are intended to answer these questions.

I shall first show how the absorption of water affects regeneration. The reader will know that from the point of view gained by a study of osmosis, the living protoplasm of plant cells is characterized by its permeability to water and its total or partial impermeability to many substances dissolved in the water. There is no reason for believing that animal protoplasm behaves any differently in this respect 
from vegetable protoplasm. If a Tubularian stem is transferred from pure sea-water to sea-water that has been diluted by the addition of distilled water, water must enter the cells, and these must in consequence become more turgid. On the other hand, if the Tubularian is placed in sea-water, the concentration of which has been raised through evaporation, the absorption of water by the animal must fall below the normal, and water must finally pass from the cells into the solution. The following experiments show how changes in the concentration of the sea-water affect regeneration. The temperature in all these experiments varied between $12^{\circ}$ and $15^{\circ} \mathrm{C}$., and was always the same for the same series of experiments. The number of animals in the individual dishes of the same series of experiments was also nearly equal; every dish contained 300 c.c. of sea-water.

2. I distributed a large number of fresh, healthy stems of the same colony of Tubularia into three salt solutions. The first of these consisted of sea-water to which $33 \frac{1}{3}$ per cent. of its volume of distilled water had been added; the second, of ordinary sea-water; and the third, of sea-water which had been evaporated to 75 per cent. of its volume. In order to have all the solutions exactly alike except in concentration, the two former were heated to the boiling-point, filtered, and, like the third, shaken for some time in the air. After two days nine of the twelve stems in the dilute seawater had regenerated the polyps which they had lost; in the normal sea-water only six of the sixteen stems had regenerated; in the concentrated sea-water regeneration had not taken place in a single stem. Six hours later regeneration was complete in all the Tubularians in the dilute seawater, and on the following day this was also the case in the ordinary sea-water. Three days later a change occurred at the cut end of one of the animals in the concentrated salt solution which looked like a beginning of regeneration, but it 
went no farther. I now divided the concentrated sea-water and the animals contained in it between two vessels, and added enough distilled water to one of them to restore it to the concentration of normal sea-water. After a few days all the animals in this vessel regenerated their polyps in an entirely normal manner, but no regeneration whatsoever occurred in the other. I have repeated this experiment several times, always with the same result. While, therefore, a great lowering of the concentration of the sea-water $\left(33 \frac{1}{3}\right.$ per cent.) not only does not inhibit regeneration, but may accelerate it, an increase in the concentration to a like amount suffices to prevent regeneration altogether, or almost entirely, without, however, injuring the power of regeneration, provided the animal does not remain too long in the concentrated solution.

3. In order to determine the limit of concentration in which regeneration is possible, I evaporated normal sea-water to $90,80,75,70,60$, and 50 per cent. of its volume. The solutions were filtered and shaken for some time in the air. Regeneration took place in the first three solutions, but not in the last three. Regeneration, however, did not occur simultaneously in the first three solutions, but the more slowly, the more concentrated the solution. After lying for several days in the 70 per cent. solution, the Tubulariæ had not lost their power of regeneration, but in the stronger solutions even this had suffered. When the Tubularians were brought back from the stronger solutions into normal sea-water, they no longer formed polyps. The tissues of the stem were shrunken and often separated from the periderm. Upon the other hand, when I added as much as 50 per cent. of distilled water to ordinary sea-water, a distinct inhibition of the process of regeneration could not once be noted; only upon the addition of 100 per cent. of distilled water did regeneration cease. If still more distilled water, 
about 200 per cent., is added to the sea-water, a remarkable phenomenon occurs: large pieces of protoplasm escape from the Tubulariæ without losing their form. They are surrounded by a transparent membrane which is formed perhaps through their contact with the sea-water. I saw a piece 1-2 cm. long escape from a Tubularian in this way.

4. That an increased absorption of water by the tissues favors regeneration when it does not exceed a slight amount; while a greater absorption is harmful, might seem contradictory. But it is an entirely general and well-known fact that when water enters cells in too large quantities it acts as a poison. Hoppe-Seyler, for instance, attributes the death of frozen plants which are thawed out too rapidly to the fact that "in freezing, the water separates to a large extent from the solids and collects in crystals. When thawed out rapidly, the particles of solid matter lying nearest these crystals are flooded with water."

5. The pieces regenerating in the highly dilute solutions often show changes which indicate the great turgidity of the tissues, in consequence of the abnormally great absorption of water. Before the polyp is formed, globular excrescences appear at the cut ends, and the new polyps are thicker and much more nearly globular than the normal polyps. The opposite phenomena are noted in the strongly concentrated salt solutions, in which polyps remain exceedingly small.

6. In conclusion I wish to give a few figures on the relation between regeneration and the absorption of water. In order not to repeat what has already been said, I shall take the figures from experiments in which the concentration of the sea-water was increased by the addition of sodium chloride, or decreased by the addition of fresh water (Serino water). According to Forchhammer, the amount of salt

1 Hoppe-Seyler, Physiologische Chemie (Berlin, 1877), p. 30. 
contained in the sea-water taken from the ocean in the vicinity of Naples is about 3.8 per cent., of which about 3 per cent. is sodium chloride. ${ }^{1}$ The following table shows how with an increase in the amount of $\mathrm{NaCl}$ regeneration is inhibited more and more, and finally prevented altogether. The figures in the first horizontal line of the table indicate how many grams of $\mathrm{NaCl}$ were added to each 100 c.c. of sea-water. In the first vertical line are indicated the number of days that have elapsed since the introduction of the Tubulariæ into the solutions. In the horizontal lines after each date are given the number of polyps regenerated up to that date. The stems were all taken from the same colony, and each solution contained twelve stems.

TABLE I

\begin{tabular}{|c|c|c|c|c|c|}
\hline Date & $\begin{array}{c}\text { Normal } \\
\text { Sea-Water }\end{array}$ & $\begin{array}{l}0.6 \underset{\text { g }}{\mathrm{NaC}} \\
\mathrm{Na}\end{array}$ & $\begin{array}{l}10 \mathrm{~g} . \\
\mathrm{NaCl}\end{array}$ & $\begin{array}{l}1.3 \mathrm{~g} \\
\mathrm{NaCl}\end{array}$ & $\begin{array}{l}1.6 \mathrm{~g} . \\
\mathrm{NaCl}\end{array}$ \\
\hline 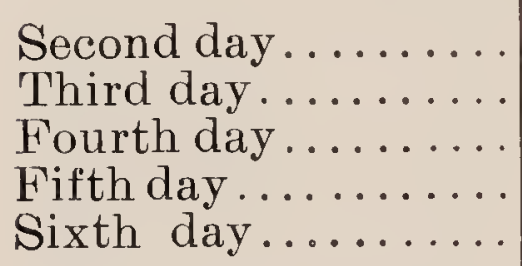 & $\begin{array}{r}4 \\
10 \\
10 \\
12 \\
12\end{array}$ & $\begin{array}{r}1 \\
2 \\
8 \\
10 \\
11\end{array}$ & $\begin{array}{l}0 \\
0 \\
0 \\
5 \\
5\end{array}$ & $\begin{array}{l}0 \\
0 \\
0 \\
1 \\
1\end{array}$ & $\begin{array}{l}0 \\
0 \\
0 \\
0 \\
0\end{array}$ \\
\hline
\end{tabular}

In order not to make the table too long, I will add that no regeneration whatsoever occurred in the most concentrated of these solutions - that in which $1.6 \mathrm{~g}$. of sodium chloride had been added to each 100 c.c. of sea-water. The retardation of regeneration was well marked, even when only 1 per cent. $\mathrm{NaCl}$ was added to the sea-water, and even in such a solution not all the animals regenerated. The repetition of these experiments yielded the same results.

The concentration in which no regeneration occurs is therefore reached when $1.6 \mathrm{~g}$. of $\mathrm{NaCl}$ is added to 100 c.c. of sea-water. This value corresponds to a concentration obtained by evaporating 100 c.c. of sea-water to 69 c.c., if the entire

1 Rотн, Allgemeine und Chemische Geologie, Vol. I, p. 524. 
amount of salt contained in the sea-water is counted as $\mathrm{NaCl}$. Our previous experiments had, indeed, shown that the concentration limit for regeneration in Tubulariae is reached when 100 c.c. of sea-water is evaporated to 70 c.c.

The second table gives the results of an experiment which shows the difference between the effect of increasing and decreasing the concentration of the sea-water. Each solution contained six animals. The percentages indicate the volume of normal sea-water, + indicating an increase, indicating a decrease, in the amount of water contained in it. The figures indicate the percentage by which the original volume has been increased or decreased.

TABLE II

\begin{tabular}{c|c|c|c|c|c|c|c|c}
\hline Date & $-26 \%$ & $-21 \%$ & $-8 \%$ & $\begin{array}{c}\text { Normal } \\
\text { Sea-W't'r }\end{array}$ & $+60 \%$ & $+50 \%$ & $+70 \%$ & $+100 \%$ \\
\hline Third day.... & 0 & 1 & 2 & 4 & 4 & 1 & 0 & 0 \\
Fourth day.... & 0 & 5 & 6 & 6 & 6 & 6 & 5 & 4 \\
Fifth day.... & 3 & 6 & 6 & 6 & 6 & 6 & 6 & 6 \\
\hline
\end{tabular}

I continued my observations for two weeks, but no regeneration occurred in the most concentrated of these solutions (-26 per cent.) beyond the three animals mentioned in the table.

7. The facts of this chapter may be shortly summed up in this: an absorption of water is essential to regeneration in Tubularia (and probably all animals). If the absorption of water is limited by keeping Tubularia in concentrated seawater, regeneration is retarded, and finally completely inhibited, by even a slight increase in the concentration. Regeneration is, however, not only not retarded but, if anything, accelerated, if the Tubularians are put into diluted sea-water. Only when the dilution exceeds a certain limit and the tissues are flooded with water a retardation of the regeneration occurs. While a decrease of 30 per cent. in the orginal 
volume of water contained in ordinary sea-water renders regeneration impossible, the same effect is not obtained until 125 per cent. of fresh water has been added.

IX. THE RELATION BETWEEN LONGITUDINAL GROWTH AND THE CONCENTRATION OF THE SEA-WATER IN TUBULARIA

1. One searches modern text-books of physiology in vain for a chapter on growth; it scarcely exists even by title. So far as I have been able to judge from the literature, observations on the physiological conditions necessary for growth in animals have been exceedingly few. I will give here what I have been able to find.

The oldest observations on the growth of animals are probably made by Bonnet, who was encouraged to do so through Hales's work. ${ }^{1}$ Bonnet measured the growth of worms. The choice of material for these experiments was unfortunate, as the length of these animals is subject to great variations because of the contractions of the body. Bonnet measured with calipers "la plus grande longueur du ver," and made these values the basis of his conclusions. He cut a worm into two, a second into four, a third into eight parts, etc., and tried to see whether the growth of the parts differed. His measurements showed "qu'il n'y a pas de différence considérable entre le progrès que font dans le même temps des moitiés et des quarts et ceux de huitièmes et de dixièmes" (p. 214); and further, "que la dernière portion est celle de toutes qui, en temps égal, prend le moins d'accroissement, et après elle, celles qui la précèdent immédiatement." It seems strange that the work of Bonnet stimulated no one to repeat his experiments upon more suitable material and with a better guarantee of accuracy.

So far as I can determine, further experiments on the conditions for the longitudinal growth of animals have been

1 Charles Bonnet, Euvres d 'histoire naturelle et de philosophie, Vol. I, "Traité d'Insectologie " (Neuchâtel, 1779), p. 193. 
made only by Semper. ${ }^{1}$ Semper started with the idea expressed by H. Spencer in his Principles of Biology: "All who have had experience in fishing in the Highland lochs know that where the trout are numerous they are small, and where they are comparatively large they are comparatively few." Semper studied the effect of the quantity of water upon the longitudinal growth of Lymnaeus stagnalis. Elodea canadensis furnished in abundance served as food. The stiff shells of the Lymnaei allowed an accurate measurement of the amount of growth. Semper found that when the food was equally overabundant the Lymnaei grew the more rapidly, within certain limits, the greater the amount of water in which they were contained. With an increase in the volume of water from 100 c.c. to 500 c.c. the longitudinal growth increased rapidly. As soon as the volume of water amounted to 5,000 c.c., a further increase had no effect upon growth.

After sixty-five days in one experiment, a shell

\begin{tabular}{|c|c|c|c|c|c|c|c|}
\hline 100 & c.e. & of & rat & was & & nn & ong \\
\hline 250 & " & " & "6 & " & 9 & " & " \\
\hline 600 & $"$ & " & $"$ & " & 12 & " & \\
\hline 2,000 & 6 & " & 6 & $"$ & 18 & “ & \\
\hline
\end{tabular}

Semper is inclined to believe that the dependence of the growth upon the amount of water present is attributable to the presence of a substance in the water "without which the other conditions for growth, even when present, can have no effect upon growth." In his book on The Conditions of the Existence of Animals, he describes further experiments in this direction:

The salts in the water, the presence of which can be demonstrated chemically, cannot be the cause of the stunted development. With the friendly aid of a chemist, Professor Hilger of Erlangen, I repeated my experiments with distilled water, or with water in

1 C. Semper, "Ueber die Wachstumsbedingungen des Lymnaeus stagnalis," Arbeiten aus dem Zool. zootom. Institut in Würzburg, Vol. I (Würzburg, 1874), p. 137; and Die naturalischen Existenzbedingungen der Thiere, Part I (Leipzig, 1880), p. 195. 
which the substances contained naturally (calcium carbonate, magnesium sulphate, etc.) were present to the point of saturation. The course of the experiments always showed that the salts contained in the water, and the presence of which had been demonstrated chemically had no particular effect.

In spite of this, Semper concludes

that some substance must be present in the water, probably in exceedingly small amounts, which, through its solution in the water, and its osmotic behavior toward the skin of the animal, is absorbed by the latter in definite, though perhaps small, amounts.

It seems to me that an important factor has not been recognized in these experiments. An excess of food (Elodea canadensis) was probably present in all the dishes, but it was not observed whether all the animals ate the same amounts - a factor upon which the results depend altogether. When I was raising butterflies I noticed how readily young caterpillars lose their appetites (especially immediately after hatching). In such cases the growth of the caterpillars of the same brood varies according to the amount of food they take up. It is quite possible that, if this point is taken into consideration, the experiments of Semper find a simple explanation.

I undertook my own experiments on growth to determine, first of all, whether the mechanics of growth is the same in animals as in plants. In plants it is believed that water enters the cells osmotically, that the cell-walls are stretched in consequence, and that through further changes this stretching of the cell-walls becomes permanent, and remains even when the intra-cellular pressure has again fallen off. If the water furnished a plant is decidedly reduced, longitudinal growth is diminished and finally stopped entirely. I have described an experiment in the preceding volume on experimental morphology which shows that those parts of the animal which have lost their turgidity are just as little able to grow as wilted plants. 
When a transverse incision abc (Fig. 59) is made transversely in a Cerianthus membranaceus fairly near the oral pole, the tentacles $T_{1}$ situated above the incision collapse like the wilted portions of the plant. When I made such a transverse incision in a Cerianthus the tentacles of which were growing actively, the tentacles

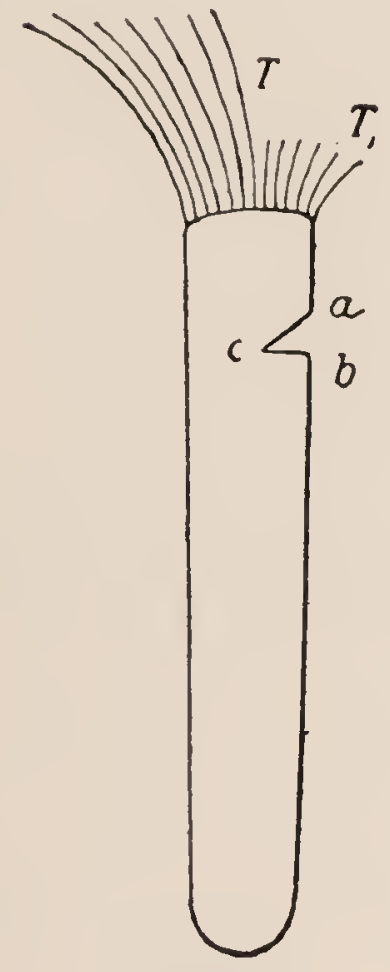

FIG. 59 above the incision ceased to grow, while the $\tau$. remaining tentacles went on. ${ }^{1}$ Cerianthus, however, could not be used for a detailed study of the dependence of animal growth upon cell turgor. Longitudinal growth cannot be measured accurately in contractile animals. Moreover, in the experiment on Cerianthus described above the amount of water absorbed by the organs cannot well be controlled experimentally. And, finally, the growth of Cerianthus is relatively slow. I therefore chose a more suitable animal upon which to experiment, namely Tubularia, and a different method of varying the amount of water absorbed - the osmotic. I am obliged to Dr. Wortmann for calling my attention to the plasmolytic experiments of de Vries.

The stem of Tubularia is surrounded by a rigid periderm, and an increase in length can be measured as accurately in this animal as in the rigid stem of a plant. I changed the concentration of the sea-water through the addition of sodium chloride or fresh water. The temperature was always the same for all the animals in the same series of experiments; the amount of liquid in each vessel was always 300 c.c.; and the number of animals in each vessel was also nearly always the same.

2. As is well known, Tubularia grows in length only when 1Sce "Heteromorphosis," Part I, p. 15. 
a polyp is present; or, what is perhaps more correct, Tubularia grows in length periodically at its oral end, every period beginning with the formation of a new polyp, and ending when this organ drops off. It is therefore necessary that all the animals of a series of experiments which are to be compared with each other should be in the same phase of growth. Since this does not necessarily occur naturally, I cut off the polyps of all of the animals when a series of experiments was started. In all these then began a new period of growth, in which a polyp was first formed, and after which the stem grew in length (the growing part of a stem being situated close behind the tip of the polyp). I waited until the polyps had dropped off in all the specimens, and then I knew that the period of growth "was at an end. I then compared the longitudinal growth in the individual specimens which had been subject to different conditions. Since growth always occurs with the formation of a new polyp, it follows, without further comment, that the concentration limits for the regeneration of the polyps are also the concentration limits for the growth of the Tubularian stem.

3. I cut pieces having about the same length and thickness from the stems of a large number of individuals and distributed them equally into various dishes containing seawater of different concentrations. Every vessel contained seven to nine animals. After eight days, in which time they had formed new polyps and grown vigorously, the Tubularians were removed and the amount of new growth was measured. The following table shows the increase in the linear growth of the individual Tubularians. The figures of the first horizontal line show the amount of salt, in per cent., contained in the different solutions used; in the vertical line under each of these figures is given the increase in the length of the individual Tubularians. 
TABLE III

\begin{tabular}{|c|c|c|c|c|c|c|c|c|c|}
\hline $5.1 \%$ & $4.8 \%$ & $4.4 \%$ & $+.1 \%$ & $\begin{array}{c}3.8 \% \\
\text { (Normal } \\
\text { Sea-Wat'r) }\end{array}$ & $3.2 \%$ & $2.5 \%$ & $1.9 \%$ & $1.6 \%$ & $1.3 \%$ \\
\hline $\begin{array}{l}\mathrm{mm} . \\
1 \\
\mathbf{1} \\
0.5 \\
0 \\
0 \\
0 \\
0 \\
. \\
.\end{array}$ & $\begin{array}{c}\mathrm{mm}_{2} . \\
2 \\
2 \\
3 \\
1 \\
1 \\
0 \\
0 \\
0 \\
. .\end{array}$ & $\begin{array}{c}\mathrm{mm} . \\
3 \\
4 \\
2 \\
3.5 \\
2 \\
6 \\
2 \\
\cdots \\
. .\end{array}$ & $\begin{array}{c}\mathrm{mm} . \\
11 \\
5 \\
3 \\
2 \\
2 \\
3 \\
3 \\
3 \\
4 \\
1 \\
0\end{array}$ & $\begin{array}{c}\mathrm{mm} . \\
\mathbf{1 0} \\
5 \\
5 \\
6 \\
3 \\
2 \\
4 \\
0 \\
. .\end{array}$ & $\begin{array}{c}\mathrm{mm} . \\
13 \\
7 \\
6 \\
1 \\
5 \\
5 \\
5 \\
. \\
.\end{array}$ & $\begin{array}{r}\mathrm{mm} . \\
19 \\
13 \\
8 \\
7 \\
11 \\
12 \\
4 \\
0 \\
. .\end{array}$ & $\begin{array}{c}\mathrm{mm} . \\
5 \\
5 \\
4 \\
3 \\
5 \\
3.5 \\
8 \\
2 \\
. .\end{array}$ & $\begin{array}{c}\mathrm{mm} . \\
1 \\
0.5 \\
0 . \overline{5} \\
0 \\
0 \\
0 \\
. . \\
. \\
. .\end{array}$ & $\begin{array}{c}\mathrm{mm} . \\
0 \\
0 \\
0 \\
0 \\
0 \\
0 \\
0 \\
. \\
.\end{array}$ \\
\hline$A \nabla \ldots 0.3$ & 1.1 & 3.2 & 3.8 & 4.4 & 6 & 10.6 & 4.4 & 0.3 & 0 \\
\hline
\end{tabular}

We obtain a better view of these results when we present them graphically. In the curve shown in Fig. 60 of the text the different amounts of salt contained in each 100 c.c. of the

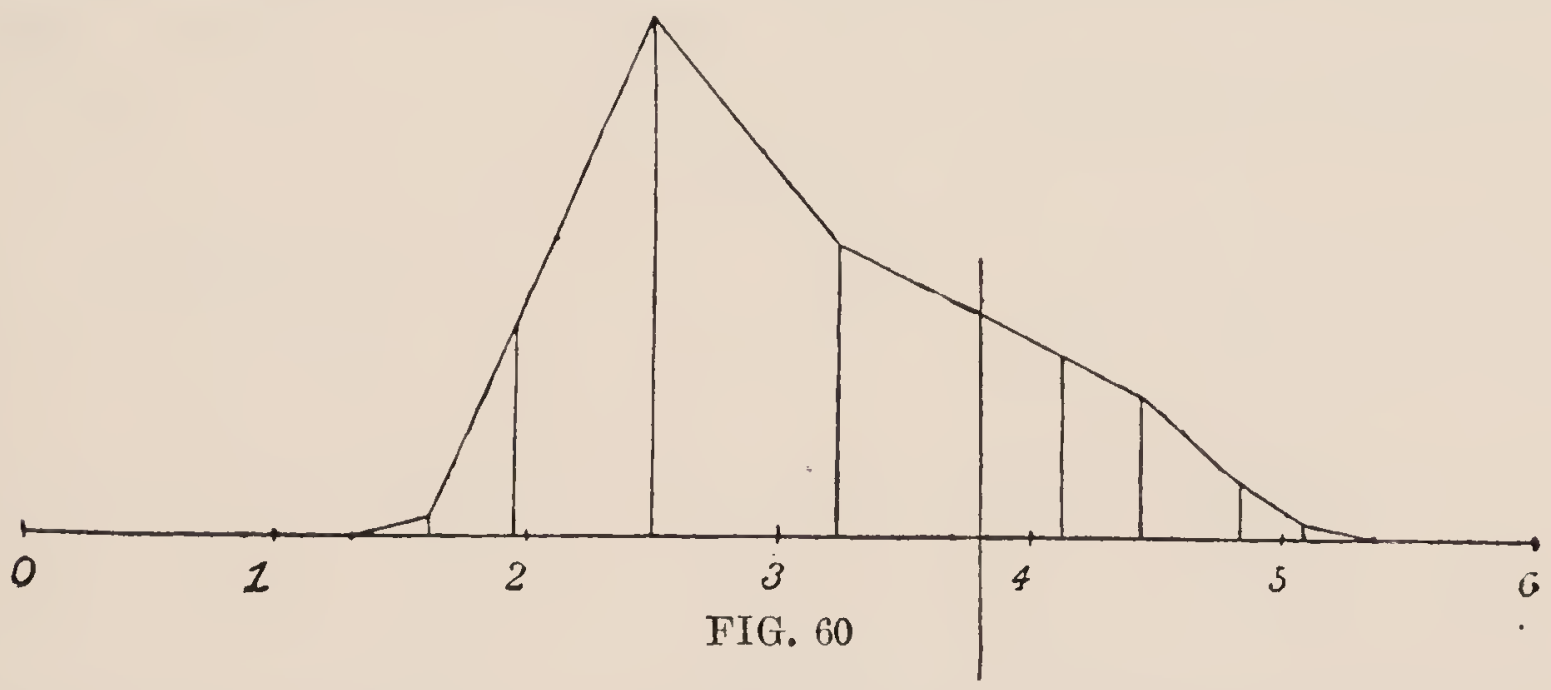

various solutions are represented on the axis of abscissas, the increase in the length of the animals on the ordinates. We see that the growth is nil in a 1.3 per cent. salt solution; that it is just perceptible in a 1.6 per cent. solution; that it increases very rapidly with an increase in the concentration, attaining a maximum in a 2.5 per cent. solution, and then decreases slowly with a further increase in the concentration. Maximal linear grouth does not occur in ordinury sea-water, but in markedly diluted sec-water. The meaning of the curve is very simple: the cells of Tubularia must be turgid in order to grow, and this is possible only as long as the 
concentration of the solution in which they are contained does not exceed a certain limit; this limit is attained when $1.6 \mathrm{~g}$. of $\mathrm{NaCl}$ is added to 100 c.c. of sea-water-when the solution contains 5.4 per cent. salt. As the concentration decreases, the Tubularian stem must absorb more and more water from the solution, and we therefore find linear growth to increase with a decrease in the amount of salt in the solution, until the limit is reached where the poisonous effects of the large amount of water show themselves. From this point on a further increase in the amount of water contained in the animal must cause growth to fall rapidly to zero. This accounts for the rapid fall in the curve between the values 1.9 and 2.5 of the abscissa.

A second experiment, performed under entirely similar conditions, yielded the same result as the above. This is shown by Table IV.

\section{TABLE IV}

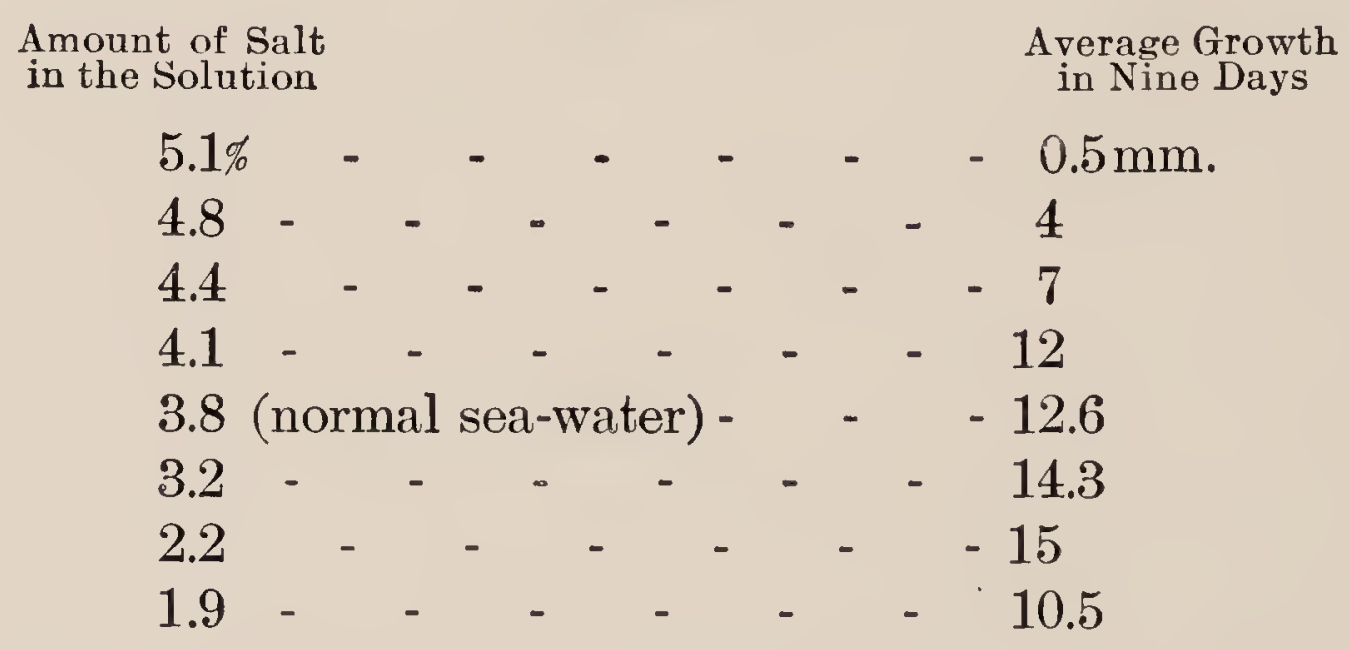

As in the preceding experiment, linear growth increases in this case also with a decrease in the concentration, attaining a maximum, not in ordinary sea-water but in a more dilute salt solution about 2.2 per cent. Beyond this point growth falls rapidly. In other experiments also-which, however, I omit here because I failed to measure the increase in the length of all the specimens, and therefore cannot tabulate them - I was able to show that the relation be- 
tween growth and the amount of water in the solution was in all points essentially the same as detailed here. We found in a preceding chapter that the polyp is regenerated later in a very highly concentrated or a very dilute solution than in a solution the concentration of which lies between these two extremes. We have therefore to determine in how far this circumstance compels us to make corrections in the experimental results given above. Between the concentrations 4.4-2.5 per cent. the difference in time between the formation of polyps in the different concentrations is so slight that they need not be considered. So far as the very concentrated solutions, 5.1 and 4.6 per cent., are concerned, I have made experiments which I have continued for weeks and months, and have found that the absolute increase in length during this time is practically zero, even though the animals formed new polyps repeatedly during this time.

4. I have not made any measurements on the increase in the thickness of Tubularia. Yet the effect of the concentration of the salt solution upon the diameter of the newly formed stem was very apparent even without measurements. The new stems formed in the more concentrated solutions. Those in which about $1 \mathrm{~g}$. of $\mathrm{NaCl}$ had been added to each 100 c.c. of sea-water were markedly thinner than the old ones which had been grown in ordinary sea-water. On the other hand, in the diluted sea-water the thickness of the new stems was not only not less than that of the old stems, but even greater.

5 . With an increase in the concentration of a salt solution the amount of oxygen dissolved in it decreases, and, as we shall see later that this is an important factor in regeneration and growth, we must determine whether differences in concentration influence the growth of Tubularia through their effect upon the amount of oxygen dissolved in the solutions and, if so, how much. The one direct measurement of the 
relation between the amount of oxygen dissolved in a sodiumchloride solution and its concentration that I have been able to find in the literature is the following: According to the experiments of Fernet" if I understand the term "titre de solution" correctly, the absorption coefficient

of a 5.42 per cent. $\mathrm{NaCl}$ solution at $16^{\circ} \mathrm{C} .=0.0284$

of a 0.72 per cent. $\mathrm{NaCl}$ solution at $14.1^{\circ} \mathrm{C} .=0.0293$.

The corresponding coefficients for distilled water are according to Bunsen ${ }^{2} 0.02949$ and 0.03030. The effect of concentration upon gas absorption is therefore so slight that it may be neglected in our experiments. Since data such as these are but few in number, I wish to add a few on the absorption of carbon dioxide.

Professor Zuntz, who brought Fernet's work to my notice, was so kind as to inform me that, according to his experiments, a saturated $\mathrm{NaCl}$ solution absorbs about one-third as much $\mathrm{CO}_{2}$ as distilled water. The figures of Fernet about correspond with these. According to this author, the absorption coefficient of $\mathrm{CO}_{2}$

in a 6.25 per cent. $\mathrm{NaCl}$ solution at $11.2^{\circ} \mathrm{C} .=09335$,

in distilled water (according to Bunsen) at $11^{\circ} \mathrm{C} .=1.1336$, difference $=0.2001$;

in a 2.22 per cent. $\mathrm{NaCl}$ solution at $14.1^{\circ} \mathrm{C} .=0.9463$,

in distilled water (according to Bunsen) at $14.1^{\circ} \mathrm{C} .=1.0291$; difference $=0$ 0828;

in a 0.83 per cent. $\mathrm{NaCl}$ solution at $16^{\circ} \mathrm{C} .=0.9591$, in distilled water (according to Bunsen) at $16^{\circ} \mathrm{C} .=0.9753$; difference $=0.0162$.

The decrease in the absorption coefficient of $\mathrm{CO}_{2}$ with an increase in the concentration of the $\mathrm{NaCl}$ solution from 0 to 6 per cent. is about 0.2 . This decrease about corresponds with that caused by an increase in the temperature from 10

1 Fernet, Annales des sciences naturelles, 4th Series, "Zoologie," Vol. VIII (1857). See also Zuntz, "Blutgase und resp. Gaswechsel," Hermann's Handbuch der Physiologie, Vol. IV.

2 Bunsen, Gasometrische Methoden, 2d ed., 1877. 
to $16^{\circ} \mathrm{C}$. Now, I have found that a rise of temperature of from 10 to $16^{\circ}$ not only does not diminish growth, but increases it, in spite of the fact that the Tubulariæ need more oxygen when the temperature is raised. It can therefore not be assumed that a decrease in the amount of oxygen contained in the sea-water brought about by the addition of sodium chloride in varying amounts up to $1.6 \mathrm{~g}$. to each 100 c.c. of water determines the decrease in linear growth. Professor Zuntz, to whom I appealed in the absence of more extensive experiments on the effect of concentration upon gas absorption, does not believe that so slight a difference in the amount of dissolved oxygen as was observed in the solutions employed in these experiments need be considered in my results, since animals get along well in summer, when the temperature is high and the demand for oxygen is correspondingly increased.

\section{SOME REMARKS ON THE EXPERIMENTS OF SCHMANKEWITSCH}

1. The proof which has been given in the preceding chapter that, with changes in the amount of water absorbed, the growth of animals is changed in the same way as the growths of plants, enables us, I believe, to give a physical explanation of some of the wonderful experimental results obtained by. Schmankewitsch in the artificial conversion of the genera Artemia mülhausenii, salina, and Branchipus. ${ }^{1}$

In 1871, during a flood,

the dam which separates the less salty water of the upper part of the Kujalnik Liman from the lower part, which is filled with salt precipitated from its own waters, broke through, diluting the water of the lower part to $8^{\circ}$ Beaumé, and causing a large number of Artemia salina to appear in it, which had evidently been brought down from the upper part of the Kujalnik and the salt-water pools in its vicinity. In the course of the following year the concentra-

1 IV. J. Sснмахkеwiтsch, Zeitschrift für wissenschaftliche Zoologie, Vol. XXV, Supplement (1875); ibid., Vol. XXIX (1887). 
tion of the water slowly rose to $25^{\circ}$ Beaumé, after which the salt again began to be precipitated. ${ }^{1}$

In the course of this time progressive changes occurred in Artemia salina, so that the Artemia present in the year 1874 had the characteristics of the species A. mülhausenii. These changes in detail are the following: (1) The adult animals of A. mülhausenii are not so large as the adult animals of A. salina. (2) Artemia salina has caudal bristles and caudal appendages, which are lacking in Artemia mülhausenii; as the concentration of the salt water increased, the caudal bristles became progressively smaller. (3) The surface of the gills is longer and narrower in Artemia salina than in Artemia mülhausenii. Ludwig (in Leunis's Synopsis) gives only the first two points. According to this author, the length of $\mathrm{A}$. mülhausenii is $6-8 \mathrm{~mm}$. ; that of Artemia salina, 8-10 mm. Schmankewitsch was able to convert A. salina into A. mülhausenii by increasing the amount of salt in the aquarium.

2. By growing Artemia in salt water that was gradually diluted, Schmankewitsch obtained a variety having the characteristics of the genus Branchipus Schaeff. The differences are very slight. Artemia has eight, Branchipus nine, footless terminal segments; and, what is of importance to us, Branchipus ferox attains a greater length, the less concentrated the salt water in which it lives.

3. If we do not allow ourselves to be influenced by the nomenclature of the systematist, the experiments and observations of Schmankewitsch show that the effect of the concentration of the salt shows itself most distinctly in the longitudinal growth of the entire animal and of some of its organs; and this always in such a way that with an increase in the concentration of the solution the longitudinal growth

1 Beaumés hydrometer is graduated, according to Wüllner, so that the point to which it sinks in water is marked 0 ; that to which it sinks in a solution of fifteen parts of sodium chloride and 85 parts of water, 15 . Water of $8^{\circ}$ Beaumé therefore contains about 9 per cent. salt; that of $25^{\wedge}$ Beaumé, about 22 per cent. 
of the entire animal or the individual organs is decreased. The result is therefore similar to that obtained in the previous experiments; wherefore I believe that the influence of concentration upon the conversion of the genera Artemia into Branchipus is to a large extent nothing but an expression of the dependence of animal growth upon the absorption of water. Schmankewitsch also mentions that in the same length of time the animals in the dilute salt solution (Branchipus) grow more rapidly than those in the concentrated salt solution (Artemia): "At the same temperature, the growth of specimens of Artemia salina in highly concentrated sea water is less than a third as great as the growth of Branchipus ferox in the less concentrated sea water." But that we are, indeed, justified in this case in substituting for the conceptions of "adaptation" and "change of species" - toward which Schmankewitsch takes a more critical view than most Darwinians - the osmotic dependence of longitudinal growth upon the concentration of the salt solution, is well shown by a fact, which is especially emphasized by Schmankewitsch, that the "change" persists in the succeeding generations only as long as the animals remain in the sea-water of the altered concentration - as should be the case if we are dealing merely with osmotic effects during the period of growth. Since not only the absorption of water, but also the secretion of water, must be considered in such experiments as have been detailed here, it is to be expected that the growth of all animals and all organs is not affected to the same extent by changes in the concentration of the salt solution.

4. Schmankewitsch interprets the effect of the concentration of the salt solution upon changes in the characteristics of the animals in a different way; he attributes it solely to the fact that with the increase in the concentration the amount of air dissolved in the salt solution is decreased, and 
its specific gravity increased. He seems to have overlooked the osmotic effects. The following indicates Schmankewitsch's (physiologically untenable) conception of the effect of the air dissolved in the salt water: "If the salt water is not diluted very gradually, the specimens of Artemia salina die of exhaustion, attributable probably to the increase of oxidations due to the greater amount of air in the more diluted sea-water." In this case we probably have to do with the fact that in the rapid dilution of the salt water the protoplasm of the cells of Artemia is suddenly flooded with water; ${ }^{1}$ it is an effect similar to that brought about by rapidly thawing out frozen organs. I will not deny, of course, that the decrease in the amount of oxygen dissolved in a salt solution can also inhibit growth when it exceeds a certain limit. This is shown very clearly by the following experiments.

\section{THE NECESSITY OF OXYGEN IN REGENERATION}

When sea-water was boiled, and Tubularian stems introduced into it after it had cooled, no regeneration of polyps occurred; if, however, I shook the boiled water for a time thoroughly with air, regeneration followed. Water therefore must contain a certain minimal concentration of oxygen in order to render regeneration possible.

But it can also be shown that the end of a Tubularian which is to regenerate must be surrounded by water containing a definite amount of oxygen, and that it is not sufficient to have merely the remaining portions of the Tubularia taking up oxygen. The bottom of an aquarium was covered with fine sand. I set glass tubes $5 \mathrm{~cm}$. long and $3-4 \mathrm{~mm}$. in diameter vertically in the sand. The upper end of the tube (Fig. 61) was drawn to a point $a$, which was just sufficiently fine to allow the stem of a Tubularian to pass

1 Recent experiments which $I$ have made point to the possibility that the rapid diffusion of salts from the animal when it is brought into more dilute solutions is, in some animals, the cause of death: [1903] 
through. I inserted into the tube a Tubularian from which I had previously cut off the root and polyp, so that the one end $b$ was in the tube and the other end $c$ was freely surrounded by water in the aquarium. A polyp was formed nearly always upon the free end $c$, but only exceptionally upon the end $b$ in the tube. The oxygen dissolved in the water within the glass tube did not suffice to render regeneration possible at the end $b$.

That regeneration only was inhibited, while the power of regeneration was preserved, is evidenced by the fact that if, after not too long a time, I brought the end $b$ back into fresh sea-water, regeneration occurred.

This also explains some of the facts mentioned in paper iv (Part I, pp. 123 and 124). I mentioned that when one end of a Tubularian is fixed in the sand, or in a narrow cleft between two slides, no regeneration occurs at this end

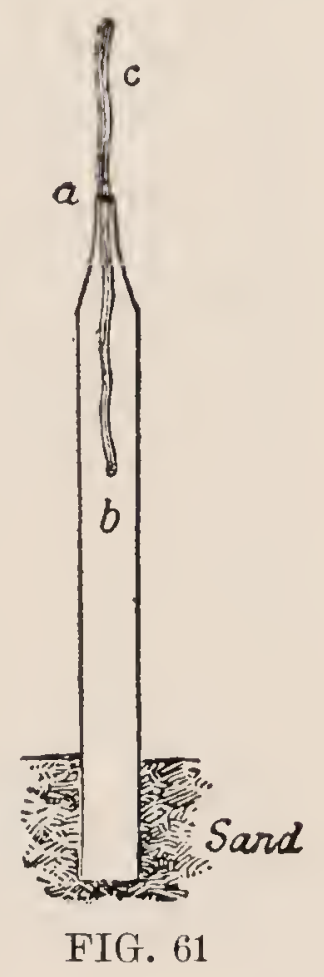
(even though death does not occur-at least for some time). At that time I was inclined to attribute the result to mechanical factors (pressure), but I believe now that we were probably dealing with lack of oxygen.

When I suspended Tubulariæ in the aquarium in such a way that the one end was very close to the surface of the sand, but did not touch it, and arranged my apparatus so that only a small current of water entered the surface of the aquarium, and kept the whole free from movement, no regeneration followed at this end. The cause for this is as above. According to Jacobsen, the layer of water just above the mud bottom of the ocean is poor in oxygen. ${ }^{1}$

It may be that a movement of protoplasm toward the end of a stem is possible only when this cut end is contained in

1 JACOBsEx, Annalen der Chemie und Pharmazie, Vol. CLXVII (1873). 
water which is rich in oxygen; in other words, that we are dealing with a case of "chemotropism."

XII. THE RELATION OF REGENERATION AND GROWTH IN TUBULARIA TO SOME OF THE INORGANIC SUBSTANCES CONTAINED IN THE SEA-WATER, ESPECIALLY POTASSIUM

1. The salts dissolved in sea-water may be of importance in regeneration and growth, not only through their osmotic effect, but also through their effect upon the metabolism of Tubularians. We have already become acquainted with their osmotic effect upon regeneration and growth. We will now investigate whether any of the substances dissolved in sea-water are indispensable for regeneration and growth in Tubularia. In the following experiments the weight of the salts always refers to the dry salt after the water of crystallization has been removed. One thousand parts of seawater, according to the analysis of Forchheimer, contain the following inorganic substances:

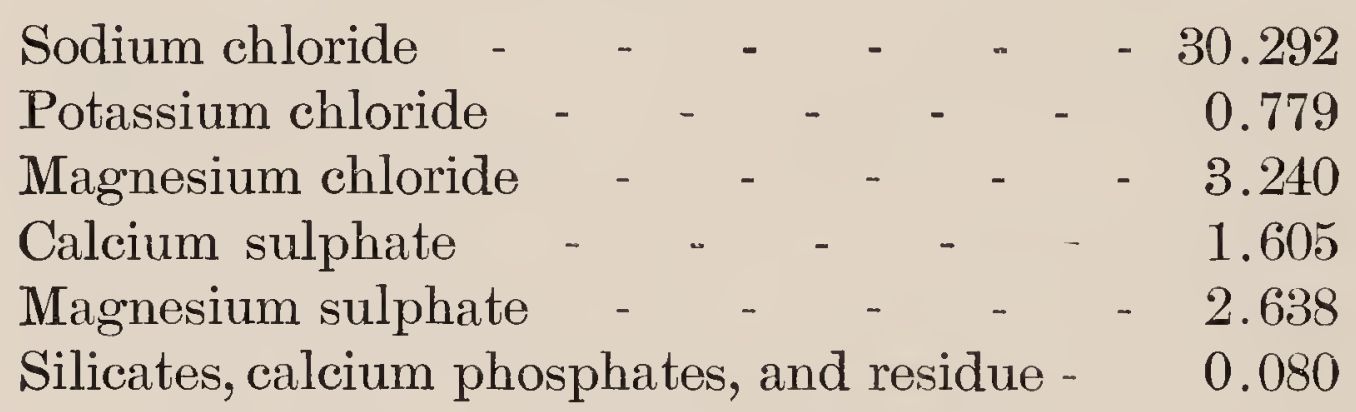

2. I added $11.3 \mathrm{~g}$. of $\mathrm{NaCl}$ to $300 \mathrm{c}$.c. of fresh water (Serino water) and the same amount to 300 c.c. of distilled water. The distilled water was thoroughly shaken in the air after the addition of the salt. The amount of salt in each of the solutions about equals that contained in ordinary sea-water. But while the Tubularians in a control dish of 300 c.c. of normal sea-water regenerated rapidly, no regeneration occurred in the animals of the same colony which were put into the pure $\mathrm{NaCl}$ solutions.

I now tried 300 c.c. of each of the following solutions: 
I

$3.3 \mathrm{~g} \cdot \mathrm{NaCl}$

100 c.c. fresh water
II

$3.3 \mathrm{~g} . \mathrm{NaCl}$

$0.03 \mathrm{~g} . \mathrm{KCl}$

100 c.c. fresh water
III
$1.6 \mathrm{~g} \cdot \mathrm{NaCl}$

$1.6 \mathrm{~g} . \mathrm{MgSO}_{4}$

$0.03 \mathrm{KCl}$

100 c.c. fresh water

No regeneration whatsoever occurred in the first solution; small and not entirely normal polyps formed in the second, but no growth occurred; normal regeneration and growth occurred in the third solution.

3. It might be possible from these experiments that the $\mathrm{MgSO}_{4}$ is essential for regeneration and growth, while $\mathrm{KCl}$ is only secondary. I therefore experimented with the following solutions:
I
$2.3 \mathrm{~g} \cdot \mathrm{NaCl}$
$1.0 \mathrm{~g} . \mathrm{MgSO}_{4}$
100 c.c. fresh water
II
$2.3 \mathrm{~g} \cdot \mathrm{NaCl}$
$1 \mathrm{~g} . \mathrm{MgSO}_{4}$
$0.03 \mathrm{~g} . \mathrm{KCl}$
100 c.c sea-water

No regeneration whatsoever occurred in the first solution; but regeneration and growth were normal in the second. After six days I divided the first solution and the animals contained in it into two vessels, adding $0.05 \mathrm{~g}$. of $\mathrm{KCl}$ to one of them, while I left the other unaltered. The animals contained in the dish to which I had subsequently added the $\mathrm{KCl}$ regenerated and grew in a normal way, while not even a suggestion of regeneration was apparent in the other. The presence of potassium in sea-water is therefore necessary for regeneration.

4. It had still to be decided which constituent of $\mathrm{MgSO}_{4}$ is essential for regeneration in Tubularia. I substituted $\mathrm{Na}_{2} \mathrm{SO}_{4}$ for $\mathrm{MgSO}_{4}$, and experimented with 300 c.c. of each of the following solutions:

I

$2.5 \mathrm{~g} \cdot \mathrm{NaCl}$

$0.8 \mathrm{~g}$. $\mathrm{Na}_{2} \mathrm{SO}_{4}$

100 c.c. fresh water

\section{II}

2.5 g. $\mathrm{NaCl}$

0.8 g. $\mathrm{Na}_{2} \mathrm{SO}_{4}$

$0.03 \mathrm{~g} . \mathrm{KCl}$

100 c.c. fresh water 
No regeneration whatsoever occurred in the first solution; in the second small abnormally formed polyps with tiny tentacles, which soon dropped off, developed. No growth occurred.

5. Whether other substances can be substituted for $\mathrm{NaCl}$ I was not able to investigate at this time. If we omit this salt from our conclusions, our experiments show that a small amount of potassium must be contained in solution if the polyps are to regenerate, but that for the formation of normal polyps and for normal growth magnesium is also required. Besides $\mathrm{NaCl}$ (for which substitutes may perhaps be found), these two substances suffice for regeneration and growth in Tubularia. ' In only one respect did the parts regenerated in my artificial solutions differ from those obtained in normal seawater--the periderm of the former remained soft, while that of the latter became hard. The importance of potassium in the formation of cells has been emphasized by HoppeSeyler :

Even though definite compounds of potassium with organic substances which might be considered essential to life-processes are unknown, the presence, without exception, of potassium in all organisms from the lowest to the highest compels us to assume that compounds of this metal play a necessary rôle in all general developmental processes. The organs of the invertebrates also always contain potassium, and those parts of plants contain the greatest amount of potassium which have the greatest developmental powers. The power of organisms to separate from and retain the potassium of the liquids which nourish them is shown especially well by the fact that fresh-water streams and lakes and sea-water contain only very small amounts of potassium beside much larger amounts of sodium, calcium, and magnesium, yet organisms growing in them contain much potassium, which they have been able to collect only from these waters which are so poor in potassium, and from nowhere else. ${ }^{2}$

1 This is not correct. The Serino water contains Ca which is also necessary for regeneration. [1903]

2 Physiologische Chemie, p. 61. 
XII. FURTHER OBSERVATIONS ON THE EFFECT OF DIFFERENT SALTS UPON REGENERATION AND GROWTH IN TUBULARIA

1. According to Hoppe-Seyler, potassium has a poisonous effect upon higher animals when introduced in too large amounts into the food. I have already shown that after the addition of 1.3 per cent. $\mathrm{NaCl}$ to sea-water, regeneration is still possible, but not growth. With this as a starting-point, I investigated whether regeneration is still possible after the addition of $0.6 \mathrm{~g} ., 1.0 \mathrm{~g} ., 1.3 \mathrm{~g}$., and $1.6 \mathrm{~g}$. of $\mathrm{KCl}$ to each 100 c.c. of ordinary sea-water. An opaque precipitate was immediately formed at both cut ends when I introduced Tubularian stems into the two most concentrated of these solutions. In not one of them did regeneration occur. After eight days I returned a number of these animals which had been in the potassium-chloride solutions to normal sea-water, in order to determine whether they were dead, or whether regeneration had only been inhibited in them. The animals returned from the 0.6 per cent. and 1.0 per cent. $\mathrm{KCl}$ solutions to normal sea-water regenerated and grew in this; the remainder, however, were dead.

I now added to each 100 c.c. of sea-water $0.16 \mathrm{~g}$. and $0.33 \mathrm{~g}$. of $\mathrm{KCl}$. In the weaker of these solutions all the animals regenerated, but much more slowly than the control animals of the same colony kept in ordinary sea-water. In the second solution only four of the nine animals regenerated. Growth was also much diminished. While longitudinal growth in the animals kept in the normal sea-water amounted to $11 \mathrm{~mm}$. upon the average, it amounted to only $1 \mathrm{~mm}$. in the same time and at the same temperature after the addition of 0.16 per cent. $\mathrm{KCl}$. No measurable growth occurred in the second solution. The addition of $0.33 \mathrm{~g}$. of $\mathrm{KCl}$ to 100 c.c. of sea-water therefore suffices to prevent growth entirely, while regeneration is not stopped until 0.6 per cent. $\mathrm{KCl}$ is 
added. In doses of $1.3 \mathrm{~g}$. to $100 \mathrm{c.c}$. of sea-water it is fatal to Tubularia.

2. $\mathrm{KNO}_{3}$ inhibited growth when $0.6 \mathrm{~g}$. was added to 100 c.c. of sea-water; regeneration was prevented by the addition of $1 \mathrm{~g}$. of $\mathrm{KNO}_{3}$ to 100 c.c. of sea-water. $\mathrm{NaNO}_{3}$ had a weaker effect; regeneration still followed the addition of 1.3 g. to 100 c.c. of sea-water; but when $1.6 \mathrm{~g}$. of this substance was added, regeneration did not occur. An experiment on growth gave the following average results; ten specimens were used in each case; the experiment lasted eleven days, and the temperature was about $16^{\circ} \mathrm{C}$.

\begin{tabular}{|c|c|}
\hline & Average Growt \\
\hline In normal sea-water & $7.3 \mathrm{~mm}$. \\
\hline Addition of 0.6 per cent. $\mathrm{NaNO}_{3}$ & 1.1 \\
\hline Addition of 1.0 per cent. $\mathrm{NaNO}_{3}-$ & -0.3 \\
\hline
\end{tabular}

The greater effect of the potassium over the sodium is less apparent in this experiment than in the chlorine compounds of this metal.

3. The poisonous action of $\mathrm{NH}_{4} \mathrm{Cl}$ upon Tubularia is very striking. An opaque precipitate is formed at both ends of a Tubularian stem when only $0.06 \mathrm{~g}$. of $\mathrm{NH}_{4} \mathrm{Cl}$ is added to 100 c.c. of sea-water. A quantity 0.03 g. to each 100 c.c. of sea-water suffices not only to inhibit all regeneration and all growth, but renders these life processes forever impossible; for when the animals are returned from such a solution to normal sea-water, they no longer regenerate and grow.

4. In conclusion I wish to direct the reader's attention to Fig. 62, which shows the regeneration and growth of three Tubularian stems taken from the same colony. They had been put into different salt solutions for the same length of time at the same temperature. $a b$ is in all cases the original piece cut from the animal. Fig. $A$ remained in ordinary sea-water. The piece $b d$ had grown at the oral end $b$, the piece $a c$ at the aboral end $a$; the polyps were very sturdy. The diameter of the new stem is nearly 
equal to that of the old. Fig. $B$ remained in sea-water to which 1.3 per cent. $\mathrm{NaCl}$ was added. Frail polyps have formed at each end with only four to six tentacles. The growth is practically zero, and the diameter of the newly grown parts is much less than that of the old stems. $C$ remained in sea-water to which 0.3 per cent. $\mathrm{KCl}$ was added. At the oral end $b$ a deformed polyp without tentacles has been formed, while no growth whatsoever has taken place. The drawing has been somewhat enlarged, and the piece $a b$ of Fig. $A$ is not shown in full length in order to save room; it was in reality as long as that shown in Figs. $B$ and $C$.

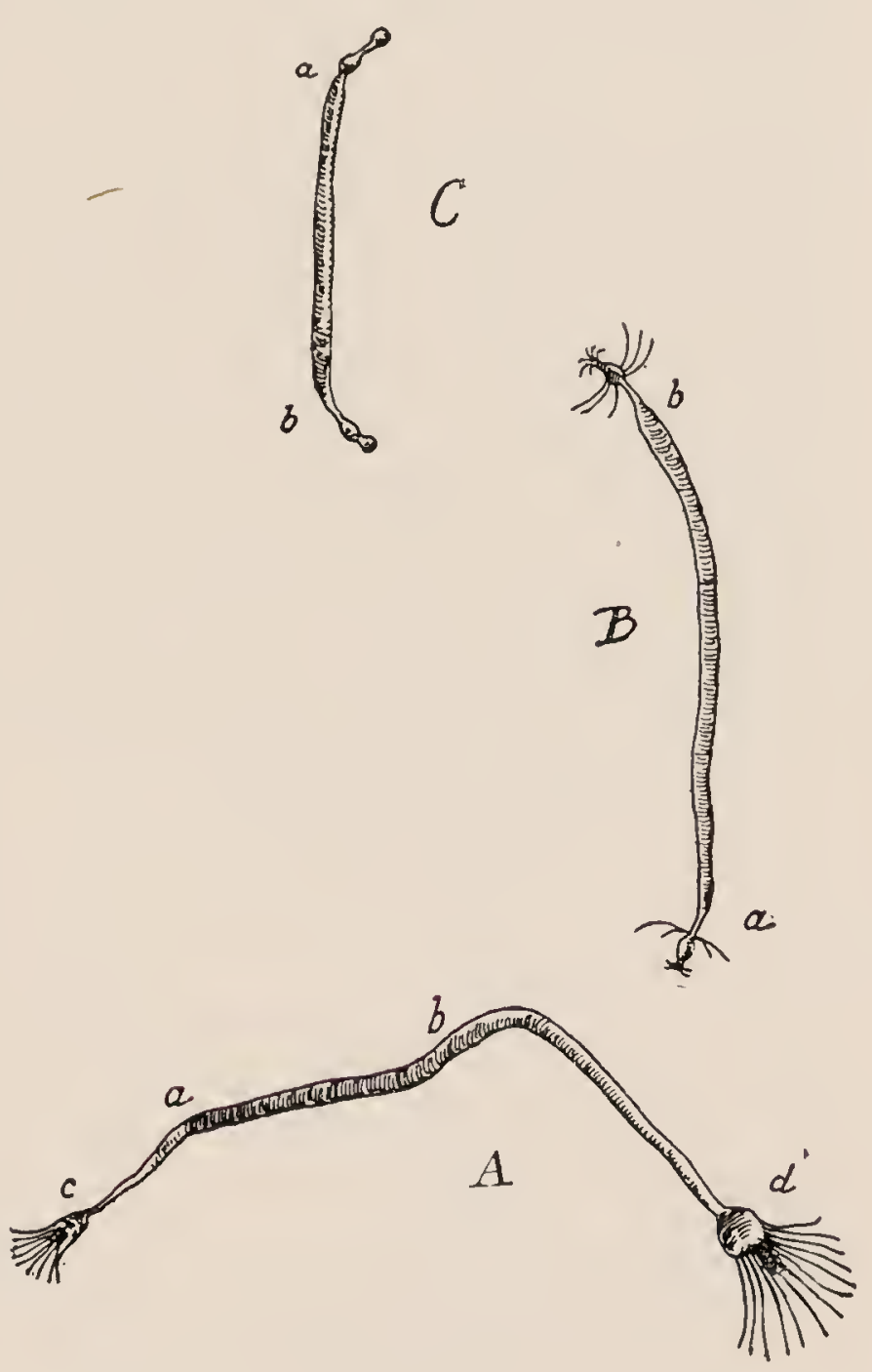

XIV. THE RELATION OF REGENERATION AND GROWTH TO THE QUANTITY OF SEAWATER

It is self-evident that for the regeneration and

FIG. 62 growth of Tubularian stems the quantity of sea-water is of importance only in so far as it enables the stems to obtain from it a sufficient amount of the inorganic substances necessary for regeneration and growth. As soon as this is the case, variations in the amount of sea-water should have no effect upon these processes.

In the experiments detailed thus far the amount of water contained in each vessel always amounted to exactly 300 c.c. 
This time I distributed the individual animals of a colony of Tubulariæ into different vessels, containing 10, 20, 50, 100, and 200 c.c. of sea-water. Each vessel contained six Tubularian stems, the roots and polyps of which had been amputated at the beginning of the experiment. The vessels were relatively flat, and only lightly covered with a glass plate to shut out particles of dust that might be carried in through the air. Oxygen could therefore readily reach the sea-water. The first polyps were formed after three days - five in each of the vessels containing 100 and 200 c.c. of sea-water, and two in each of the others. Two days later all of the animals had formed new polyps. The inhibition of regeneration was therefore only slight in the vessels containing but a small amount of sea-water. On the eighth day after the beginning of the experiment I measured the growth of the individual specimens, which was as follows:

In 10 c.c. of Sea-Water

$8.0 \mathrm{~mm}$.

12.0

6.5

5.5

7.0

4.0
In 200 c.c. of Sea-Water

$7.0 \mathrm{~mm}$.

8.0

13.0

9.0

10.0

3.0

Average $\overline{7.1} \mathrm{~mm}$.

$8.3 \mathrm{~mm}$.

I obtained about the same average values in the rest of the vessels. Ten c.c. of sea-water therefore contain sufficient inorganic material for normal regeneration and normal growth, and variations in the quantity of sea-water above this limit have no effect upon these processes. I have not made experiments with less than 10 c.c., as these barely suffice to cover the Tubularian stems. The result of these experiments is free from the complication which enters into Semper's experiments, in which the animals devoured an uncontrolled (and possibly uncontrollable) amount of vegetable food. 
XV. SOME CASUISTIC REMARKS ON HETEROMORPHOSIS

1. It was my intention to analyze the conditions underlying heteromorphosis in other forms as carefully as I have done in the case of Tubularia and Antennularia. Lack of time, however, rendered this impossible, so I was compelled to postpone these experiments. I wish, however, to add a few casual observations.

During the winter of 1889-90 I had already observed that in the aquarium the stems of Gonothyrea often grew into roots even when not injured externally. I thought at the time that lack of light and oxygen lay at the basis of these phenomena, but did not mention this fact, as I wished to make it the starting-point of new experiments. For the reasons given above, I did not succeed in mastering organization in this animal in the time at my disposal, and so have again postponed further work upon these experiments.

I have already called attention to the tendril-like bendings of the roots of Aglaophenia pluma in paper iv. These curvatures, dependent apparently upon internal causes, play perhaps a much more important rôle than I at first anticipated. They probably are responsible for the fact that the orientation of the organs, even those at a distance, does not occur with the same regularity as in Antennularia. My first experiments were made in very intense light, and it is possible that this is the determining factor in bringing about the downward growth of the adventitious roots in Aglaophenia. Considering the complexity of the conditions determining organization, experiments upon this animal with the klinostat might prove fruitful.

I found in Sertularia that new growths which had the form of roots, but were positively heliotropic, formed a polyp at their tips after they had attained a certain length, and then remained positively heliotropic. According to Sachs, certain substances are not only necessary in the formation of 
certain organs, but the specific reactions of an organ toward light and gravity are also dependent upon the nature of its substances. It might be believed, therefore, that the polypforming substances also determine positive heliotropism, while the root-forming substances determine negative heliotropism. These circumstances might therefore explain the apparent paradoxes in the reaction of Sertularia to light. I hope to be able to study this question experimentally, and therefore will not enter into any further theoretical discussion.

2. I mentioned in the previous volume of these studies that Bonnet and Dalyell had found that an organ of another kind may occasionally grow in place of one that has been lost. Dr. A. von Heider, of Gratz, called my attention to the fact that he, too, had observed and described such a case. ${ }^{1}$ I will give his description in full here:

I have often had the opportunity of testing in Cladocora the great powers of reproduction which Coelenterates in general are known to possess. Without discussing the rapid healing of wounds and the renewal of wornout portions of the body, the following case seems worthy of description. I cut off by a rapid incision, and as near the rim of the shell as possible, the polyp of a Cladocora, which was protruding a great distance beyond its shell, and allowed the animal to go on living in the aquarium. As early as the next day the tentacles of the animal, which had been robbed of its calcareous support, were entirely unfolded, the transverse wound at the opposite end had puckered to a conical scar, and the polyp moved over the bottom of the vessel by means of its tentacles. When examined with a lens some weeks later, the aboral end of the animal was completely healed and possessed of a plate running parallel to the oral plate, at the periphery of which were tiny elevations corresponding to the tentacles of the oral plate. In the course of two months these developed into full-grown tentacles. In the center of this new plate of tentacles was a round opening, the newly formed mouth, so that an entire oral plate had been formed at the cut end of the polyp, which differed in external

1 A. von HeIDer, Wiener Sitzungsberichte, Vol. LXXXIV, Part I (1881). 
appearance in no way from the old oral plate. A slight swelling of the body-wall showed the position of the original cut in this double polyp. The position of the latter also showed that the body itself had grown aborally.

\section{SUMMARY OF THE MORE IMPORTANT RESULTS}

I. The orientation of organs and the place where they originate can be controlled in Antennularia antennina at will through the following circumstances:

1. The stems are negatively, the roots positively, geotropic and positively stereotropic.

2. The place where the organs form is determined by the orientation of the animal toward the center of the earth, so that branches arise only on the upper surface of a stem; or, if the latter is in an absolutely vertical position, only from that cut end which is directed upward. The opposite holds for the roots, with this addition, however, that in the region where new stems originate new roots may at times also be formed upon the upper surface of the old stem.

3. If a growing but uninjured stem of Antennularia antennina is suspended with its tip downward, the stem ceases to grow as such, but roots may arise from the tip.

4. When a stem is placed horizontally or obliquely, the branches which are directed downward may grow as roots, even when they are not injured and not in contact with solid bodies.

II. If a piece is cut from the stem of a Tubularian, the regeneration of the polyp at the oral end may retard the formation of a polyp at the other. By suppressing the formation of the oral polyp one can accelerate considerably the formation of the polyp at the aboral end.

III. If an incision is made into one of the tubes of a Ciona intestinalis, ocellæ are formed at both edges of the wound.

IV. If the entire brain of a Ciona intestinalic is rxtir- 
pated, the reflexes are preserved, and only the threshold of stimulation for their production is raised.

$\mathrm{V}$. The brain of such an animal is regenerated in the course of a few weeks.

VI. Growth and regeneration in Tubularia is, as in plants, dependent upon the amount of water absorbed. Growth is increased by an increase in the amount of water absorbed; while it is decreased through a diminution in the amount of water absorbed. Growth is practically zero in sea-water containing 5.1 per cent. salt, though regeneration of polyps is still possible; when the water contains 5.4 per cent. salt, regeneration also is impossible. With a decrease in the concentration of the sea-water, growth becomes progressively greater, until it attains a maximum in water containing 2.5 per cent. salt. If the concentration is further diminished, growth decreases rapidly until a concentration of 1.3 per cent. is reached, when neither regeneration nor growth any longer takes place. The temperature was about $15^{\circ} \mathrm{C}$. in these experiments.

VII. When the pressure of oxygen is very low, regeneration no longer takes place; it is also necessary that the end at which regeneration is to occur be constantly surrounded by water containing a sufficient concentration of oxygen.

VIII. The salt solution in which Tubularia is to regenerate and grow must contain potassium and magnesium; yet potassium must be present only in small amounts. The addition of $0.33 \mathrm{~g}$. of $\mathrm{KCl}$ to $100 \mathrm{c.c}$. of sea-water prevents growth; an addition of $0.6 \mathrm{~g}$. to 100 c.c. of sea-water prevents regeneration also.

IX. The amount of sea-water has no noticeable effect upon growth in Tubularia so long as the animals are surrounded by a sufficient amount. 


\section{VII}

\section{EXPERIMENTS ON CLEAVAGE ${ }^{1}$}

1. Is the second part of my Untersuchungen zur physioTogischen Morphologie ${ }^{2}$ I showed that regeneration and growth in animals are, as in plants, a function of the amount of water contained in the cells. When I increased the amount of water in the cells of Hydroids by bringing these organisms into more diluted sea-water than that in which they usually live, the rate of growth increased with the decrease of the concentration of the sea-water. When I diminished the amount of water in the tissues of Hydroids by bringing these animals into a more concentrated solution than the normal sea-water, the rate of growth diminished too. We know that seedlings of plants need water in order to develop. It is the same in the animal egg, as recent investigations concerning the development of sea-urchins, starfish, arthropods, and fish showed me. If we reduce the amount of water contained in the egg of the sea-urchin by bringing it into more concentrated sea-water, the process of segmentation is retarded only as long as the increase in the concentration is small. As soon as the concentration is greater, however, the fertilized ogg does not segment at all. In one case the eggs had been fertilized at 9:40 A. м. A few minutes after the impregnation, one part $(a)$ of the eggs were put into sea-water to which $1 \mathrm{~g}$. of $\mathrm{NaCl}$ to 100 c.c. had been added. A second part $(b)$ were put into sea-water to which I had added $1.3 \mathrm{~g}$. of $\mathrm{NaCl}$ to 100 c.c. A third part $(c)$ were brought into seawater, the concentration of which was increased by the addition of $2 \mathrm{~g}$. of $\mathrm{NaCl}$ to $100 \mathrm{c.c}$; and a fourth part $(d)$

1 Journal of Morphology, Vol. VII (1892), D. 253.

2 Würzburg, 1892. Part I, p. 191. 
remained in normal sea-water. At 10:50 nearly all the eggs which had remained in normal sea-water were in the two-cell stage, while none of the eggs in the other solutions were yet segmented; in part $(a)$ the first egg was segmented at 10:55; in (b) the first segmentation took place at 11:45-nearly an hour later than in normal sea-water; and in $(c)$ no segmentation at all took place. That the amount of water and the intra-cellular pressure in these experiments varied with the concentration could be seen from the form of the cleavage spheres. In normal sea-water, and "still more in sea-water which was a little diluted by the addition of 10-20 per cent. of fresh water, the first two cleavage spheres were nearly perfect hemispheres. In sea-water of higher concentration the first two cleavage spheres became ellipsoidal in shape, approaching the sphere more the higher the concentration was. When $\mathrm{I}$ added more than $2 \mathrm{~g}$. of $\mathrm{NaCl}$ to 100 c.c. of sea-water, in a few hours plasmolysis took place, and the surface of the protoplasm began to shrink irregularly. But by bringing the eggs back into normal sea-water the normal form was restored in a few minutes.

2. Further investigations concerning this subject led me to another series of facts, which, as I believe, give the physiological explanation of some of the phenomena of cleavage. In my investigations concerning the regeneration and growth of Hydroids, I found that a salt solution which is just concentrated enough to prevent regeneration and growth by no means kills the Hydroids, or even annihilates the power of growth and regeneration. Hydroids which had been in such a solution for several days when brought back into normal sea-water began to regenerate and to grow. When I made the same experiments on fertilized eggs, the results were the same. A salt solution which is just concentrated enough to prevent segmentation does not annihilate the power of segmentation at once. But when I brought such eggs back 
into normal sea-water, I found that the manner of segmentation changes in a remarkable way, according to the time the eggs had been in the concentrated sea-water.

3. I fertilized eggs of sea-urchins at 9:30 in the morning, and at 9:43 a part of these eggs were put into sea-water to which $2 \mathrm{~g}$. of $\mathrm{NaCl}$ to 100 c.c. had been added. The rest of the eggs remained in normal sea-water. I will call the sea-water to which $2 \mathrm{~g}$. of $\mathrm{NaCl}$ to 100 c.c. had been added the concentrated solution, and the eggs which had been exposed to it the plasmolyzed eggs. At 10:20, before any segmentation even in the normal sea-water had taken place, I took a lot of eggs out of the concentrated solution and brought them back into normal sea-water. At 10:33 these eggs began to segment. The segmentation was a normal one, as only segmentation into two cells took place. At the same time segmentation had taken place in nearly all of the normal eggs. The only difference between the normal eggs and the plasmolyzed eggs was that the former at 10:33 were nearly all segmented, while of the latter only a small part had undergone segmentation. Ten minutes later, however, every second one of the plasmolyzed eggs was segmented, mostly into two, exceptionally into four, segments. But now the situation began to change. By this time the normal eggs began to reach the four-cell stage, and now many of the plasmolyzed eggs which had not yet segmented into two cells began to segment into three or four cells at once, without going through the two-cell stage at all. The cleavage took place in this way, that at the same time, or shortly after each other, spherical projections appeared on the surface of the egg, which at first were coherent, but which soon, at the same time or in quick succession, were separated. This kind of segmentation seems to be identical with that which $\mathrm{O}$. and R. Hertwig observed under other circumstances, and have described as Knospenfurchung. ${ }^{1}$ The further segmentation

1O. AND R. HERTwig, Jenaische Zeitschrift, Vol XX (1887). 
was the same in the plasmolyzed and in the normal eggs.

At 11 o'clock I brought a second lot of eggs back from the concentrated solution into normal sea-water. These eggs did not show the slightest trace of segmentation. At 11:22 the eggs began to segment, but in hardly any case did the eggs divide into two, but nearly all of them segmented into more cleavage spheres at once. The number and size of the cleavage spheres were not quite regular. There were mostly about four spheres in one egg; sometimes, however, five to eight. The size of the single cleavage spheres of the same egg varied, the smallest spheres being about the size of a cleavage sphere of the eight-cell stage, the largest that of a two-cell stage. At 11:44 the first segmentation was finished, and from now on the segmentation was perfectly regular. At 11:40 the normal eggs were in the eight-cell stage.

At 2:40 I brought another lot of eggs from the concentrated solution back into normal sea-water. Not one egg showed segmentation. At 2:50 the segmentation began. Just as in the 11 o'clock lot, hardly one egg segmented into two cleavage spheres. But while most of the eggs of the 11 o'clock lot segmented into from four to eight cells, most of the eggs segmented now into from eight to sixteen cleavage spheres at once. The number and size of the cleavage spheres varied again in the different eggs, but the striking feature this time was the prevalence of cleavage spheres of the size of the sixteen-cell stage. The normal eggs by this time were into the morula stage. At 4:05 another lot of eggs was brought back from the concentrated solution into normal sea-water. Not one egg had segmented. Twenty minutes later, however, nearly all the eggs were in cleavage. But this time they did not divide into sixteen, but into many more segments at once. I think that most of the eggs showed about thirty cleavage spheres. Of course, in this 
lot, just as in the foregoing lots of the same kind, I found cleavage spheres of very different sizes in the same egg. At 6:50 I repeated the same experiment, taking out a lot of eggs from the concentrated solution, and bringing them back into normal sea-water. Not one egg showed any trace of segmentation, but in a very short time - about twenty minutes - the eggs segmented at once into a great number of small cleavage spheres, the smallest and most numerous having the size of a cleavage sphere of about the sixty-four-cell stage. I repeated this experiment about twenty times, always with the same result, which in a few words may be expressed as follows: If we bring impregnated eggs into sea-water of a certain higher concentration, no segmentation takes place; but if we bring them back into normal sea-water, they divide in about twenty minutes directly into nearly, but not quite, so many cleavage spheres as they would contain by that time if they had remained in normal sea-water all the time. It must be added, however, that the normal eggs in this experiment are always ahead of the plasmolyzed eggs in regard to their stage of segmentation, and that their advance becomes the more obvious the farther they develop.

Eggs, after having been in the concentrated solution from twelve to twenty-four hours, do not segment at all if brought back into common sea-water. All these experiments are the more satisfactory the better the material is.

4. I varied these experiments by sometimes bringing the impregnated eggs into the concentrated solution immediately after impregnation, and sometimes later. The result remained the same, on the whole, and I will not dwell upon the details of these experiments. But the following fact may be of interest: I impregnated eggs in normal sea-water, and left them there until they were all in the two-cell stage. Then I brought them into the concentrated solution. The cleavage stopped directly. After having been there for three hours, 
I brought them back into normal sea-water; and now every cleavage sphere divided at once into more than two pieces, sometimes into eight or even more.

5. I concluded from the foregoing experiments that in the concentrated solution a segmentation of the nuclei might take place without any segmentation of the protoplasm. Eggs which had been impregnated in normal sea-water were brought into the concentrated solution and watched carefully. No segmentation of the protoplasm took place; but the nucleus divided, indeed, into two, and then further divisions followed. I tried, moreover, to see whether the protoplasm of such eggs, if brought back into normal sea-water, divided into as many cleavage spheres as there were nuclei preformed. I saw, indeed, that every nucleus becomes the center of one of these projections, which later on become cleavage spheres. Dr. Conklin was kind enough to stain some of the eggs which had been in the concentrated solution for some time and which showed no trace of segmentation. Some of these stained eggs showed very distinctly from four to about thirty distinct nuclei. In other eggs the segmentation of the nucleus was not so perfect. The nucleus, extremely enlarged, seemed to consist of several parts, which, however, were still connected. These eggs had been killed at a time when the eggs of the same lot which had remained in normal sea-water all the time were in about the sixty-fourcell stage.

6. Fol and $\mathrm{O}$. and R. Hertwig found that in the case of polyspermia the egg at once divides into about as many cells as there are asters. We know that for the segmentation of the protoplasm it does not make any difference whether the nuclei are derived from the male pronuclei exclusively, as in the case of the impregnation of an enucleated egg; or from the conjugated nuclei, as in the normal case; or from both conjugated nuclei and male pronuclei together, as in some cases 
described by Fol. In my experiments the eggs were impregnated under normal conditions, and cases of polyspermia were very rare indeed. Nearly all of the eggs which remained in normal sea-water segmented quite normally. But I thought of the possibility that new spermatozoa might enter the impregnated egg in the concentrated solution. I knew that such a supposition was in contradiction with all known facts, but these facts are still meager. If a polyspermia in my experiments took place, it could happen only in the concentrated solution, as here the increase of the number of the nuclei was observed. But I found that the spermatozoa were perfectly paralyzed as soon as they were brought into the concentrated solution; that is, in the sea-water to which $2 \mathrm{~g}$. of $\mathrm{NaCl}$ to 100 c.c. had been added. I could show, moreover, that in this concentrated solution no impregnation is effected. I brought unfertilized eggs into this concentrated solution and added spermatozoa. When I brought them back into normal sea-water, it took more time from that moment until segmentation began than it took in normal eggs and in normal sea-water from the moment of impregnation to the moment of segmentation. The spermatozoa contained in the concentrated salt solution became active again a few minutes after being brought back into normal sea-water and then entered the eggs. Polyspermia in this case could be observed, but not as a rule. Most of these eggs segmented into two cells. But it was astonishing how soon the spermatozoa lost their power of impregnating under these circumstances. Spermatozoa which had been in the concentrated solution only a few hours, when brought back into normal sea-water fertilized only a thousandth part, or still less, of the normal eggs; while spermatozoa of the same animal which had remained in normal sea-water fertilized at the same time practically all the eggs of the same female. When I tried to fertilize eggs in normal sea-water which had been in the concentrated 
solution for a few hours with spermatozoa that had been under the same conditions, only about one egg in a million began to show some trace of segmentation, and as a rule this segmentation remained in statu nascendi, but was not accomplished. All these observations are totally different from the phenomena described above. Eggs which had been fertilized in normal sea-water, and which were put into the concentrated solution, after being brought back into normal sea-water for from ten to twenty minutes segmented without any exception, and were able to develop into normal blastulae and plutei. Eggs of this kind were still able to develop into normal larvæ after having been in the concentrated solution for four to six hours. But eggs which before impregnation had been put into the concentrated solution together with spermatozoa, and which four to six hours later were brought back into normal sea-water, reached only the first stages of segmentation, if they segmented at all, and then stopped developing. I never got a living larva from these eggs. From all these facts I conclude that the continual increase of the nuclei of the impregnated eggs in the concentrated solution was due, not to polyspermia, but simply to segmentation of the nucleus. In these experiments bacteriological precautions are necessary, as the water of the aquarium is liable to contain quantities of spermatozoa.

7. From the above I believe to have shown that by bringing fertilized eggs of sea-urchins into more concentrated seawater-we added 2 to $2.4 \mathrm{~g}$. of $\mathrm{NaCl}$ to 100 c.c. of sea-water - the segmentation of the nucleus proceeds; although more slowly than under normal conditions, while no segmentation of the protoplasm is possible. The fact in itself is of some technical value, as it enables us to separate two processes which nature generally produces together, or which hitherto we had not the power to separate at desire. In regard to our knowledge of segmentation, we see from this that the 
physiological conditions for segmentation of the nucleus are different from the physiological conditions of the segmentation of the protoplasm. We now can be positive in this regard, as under the same conditions the nucleus continues segmenting, while the protoplasm does not show the slightest trace of segmentation. But these experiments allow us to go one step farther and to make clear one element in the complex called segmentation, namely, the physiological cause for the segmentation of the protoplasm. We saw that in the concentrated solution the protoplasm did not segment, while as soon as it was brought back into the normal sea-water it segmented at once into about as many cleavage spheres as nuclei were formed. All further inferences depend upon our knowledge of the effect of salt solutions on protoplasm. I have investigated this point myself, and have caused others also to take up this question. The result of all investigations hitherto carried on is as follows: Raising the concentration of the salt solution in which an animal or a tissue lives has the same effect as lowering the temperature; lowering the concentration has qualitatively and quantitatively the same effect as raising the temperature. I will mention two cases to illustrate this. First, one example to show the parallelism of the mentioned effect of the temperature and the concentration in qualitative regard. I have recently succeeded in making animals belonging to different classes-larvæ of Polygordius, Copepods, etc.- positively heliotropic by bringing them into low temperatures, and making them negatively heliotropic by raising the temperature of the water. In water from $0^{\circ}$ to about $10^{\circ}$ larvæ of Polygordius, for instance, are exclusively positively heliotropic. In water above $25^{\circ}$ they are exclusively negatively heliotropic. But by adding a certain amount of $\mathrm{NaCl}$ to normal sea-water I was able to make them just as well positively heliotropic, and by adding a certain amount of fresh water to the normal sea-water I 
could make them negatively heliotropic. The same was the case in Copepods, only the absolute figures differ, as was to be expected. By bringing living tissues into a solution of higher concentration, we reduce the irritability by reducing the amount of water contained in them. By reduction of irritability we mean that the effect determined by the same cause is quantitatively less. That explains how the segmentation of the protoplasm is generally determined, why in a solution of a certain concentration no segmentation of the protoplasm takes place, and why when brought back into normal sea-water the protoplasm segments at once into about as many spheres as there are nuclei preformed. The segmentation of the protoplasm is the effect of a stimulus which the nucleus applies to the protoplasm, and which makes the protoplasm close around the nucleus. If we bring the fertilized egg in the concentrated salt solution ( $2 \mathrm{~g}$. of $\mathrm{NaCl}$ to 100 c.c. of sea-water), the nucleus divides, and every nucleus applies the stimulus to the protoplasm with which it is in contact. But the protoplasm of the egg, on account of its containing too little water, is in the condition of a cooled-off muscle, which does not answer to the stimulation of the nerve, and no segmentation of the protoplasm takes place. But as soon as we bring the egg back into normal sea-water, the protoplasm takes up water very fast and regains its irritability; and now, of course, it answers to the stimuli from the nuclei, and closes around every nucleus of segments. If we add a smaller dose of $\mathrm{NaCl}-$ namely $1.3 \mathrm{~g}$. of $\mathrm{NaCl}$ to 100 c.c. of sea-water-the irritability is only a little less than it is normally, and the whole effect is that the reactions of the protoplasm are somewhat slower and retarded. Of what kind the stimulus is, and from which part of the nucleus it is exercised, we cannot tell. From other facts I am inclined to believe that this stimulus is a chemical one, and caused by certain substances produced in 
the nucleus which also may be effective if separated from the nucleus.

8. The physiological causes of the segmentation of the nucleus are not directly touched by these experiments. But two points ought to be mentioned: first, that the segmentation of the nucleus in the concentrated solution ( $2 \mathrm{~g}$. of $\mathrm{NaCl}$ to 100 c.c. of sea-water) was retarded, and at last ceased entirely after from twelve to twenty-four hours; secondly, that the segmentation of the nucleus was extremely irregular when the protoplasm did not take part in segmentation. We see in these facts some of the influences which the protoplasm exercises on the segmentation of the nucleus. This influence may be exercised in this way, that by the high intra-cellular pressure which normally exists in the cleavage spheres these spheres press and flatten each other. The form of the cell, however, determines, as Sachs showed long ago, the orientation of the plane of division, and, as Hertwig believes, in such a way that the longitudinal axis of the Kernspindel is put in the longest diameter of the cell. Therefore we ought to expect that, within certain limits, with increasing intracellular pressure, Sach's law of the rectangular division of the planes of cleavage would become more obvious. I found, indeed, that in normal, or still more in somewhat diluted, sea-water, where the turgor, and consequently the flattening of the cleavage spheres, was the greatest, Sach's law was the most exactly realized. Therefore this geometrical regularity in the segmentation of the nucleus which is so striking under normal conditions must disappear at once if the protoplasm does not take part in segmentation.

9. Our observations concerning the dependence of irritability of the protoplasm upon the water contained in the tissues add one mcre fact to those given already to explain the importance of water for all processes of growth and development. If we reduce the amount of water in a regenerating or 
growing tissue, we not only retard or prevent these processes by reducing the volume of the cells and the mechanical effects of the intracellular pressure, but we reduce also the irritability of the protoplasm. This irritability, as we saw, plays an important rôle in the process of cleavage, and as regeneration and growth is a function of processes of cleavage, we at once understand why regeneration and growth must be retarded or accelerated by bringing Hydroids into more concentrated, or more diluted, sea-water. But if this inference is right, our experiment holds good for the process of cleavage not only in eggs, but in cells in general.

The experiments which are mentioned in this paper were all made on sea-urchins (Arbacia).

The chief result of these investigations is, shortly, as follows.

If we reduce the irritability of the protoplasm of the egg by reducing the amount of water contained in it, the nucleus can segment without segmentation of the protoplasm. If we increase again later the amount of water, and consequently the irritability of such an egg, the protoplasm at once divides into about as many cleavage spheres as there are nuclei preformed. The segmentation of the protoplasm in the egg, and probably in every cell, is only the effect of a stimulus exercised as a rule by the nuclei. 


\section{VIII}

\section{THE ARTIFICIAL TRANSFORMATION OF POSITIVELY HELIOTROPIC ANIMALS INTO NEGATIVELY HELIO- TROPIC AND VICE VERSA ${ }^{1}$}

THE new facts contained in the-following pages deal chiefly with the task of rendering positively heliotropic animals negatively heliotropic, and vice versa. I think also that I have discovered a difference in positively and in negatively heliotropic animals with regard to the liberation of energy. As both series of observations may give us some clue in regard to the nature of heliotropic phenomena in general, I have briefly repeated here the description of the simple facts of heliotropism, and have prefaced it with a short theoretical explanation. A later part in this paper treats of the behavior of animals, which, though not heliotropic, still react to the light by movements. These I shall term photokinetic (unterschiedsempfindlich). In the concluding part of this paper are given the results of some further experiments bearing on the causes of depth-migration and depth-distribution in marine animals.

\section{THE SIMPLE FACTS OF HELIOTROPISM}

1. All former authors who have studied the behavior of animals toward light have, without exception, been of the opinion that animals "preferred" either light or darkness, and correspondingly either sought the light places in space or shunned them. Five years ago I showed that there is a large number of animals which are oriented by the light, and in such a way that they are forced to place their axes or planes of symmetry in the direction of the rays of light.

1 Pfügers Archiv, Vol. LIV (1893), p. 81. 
This leaves still two possibilities: the oral, or the aboral, pole may be turned to the source of light. When the former is the case, the animals are called positively heliotropic; when the latter is the case, negatively heliotropic. In the case of sessile animals orientation was brought about by the light without any complicating secondary phenomena, and when light fell upon them from one side only, heliotropic curvatures resulted just as in plants. Spirographis spallanzanii gave rise to positively heliotropic curvatures; while the stolons of Sertularia gave rise to negatively heliotropic curvatures under certain conditions. If, however, the animals are able to move freely, a complicating feature appears, inasmuch as the animals execute progressive movements, and these take place in the direction of the rays of light, as the median plane of the animals is brought into this direction. If the animals are positively heliotropic, progressive movements must occur toward the source of light. If the animals are negatively heliotropic, they must move away from the light. The difference between. this idea and that of former authors is recognized immediately. According to my idea, the fact whether the animals go toward the light or away from it, is a consequence of their orientation by the light — a fact which former authors overlooked. Moreover, the direction of the progressive heliotropic movements lies in the direction of the rays of light-another fact which had been universally overlooked. The former conception, that certain animals seek the "light," while others seek the "darkness," is completely refuted by the fact, which I discovered, that positively heliotropic animals can be forced to go in the direction of the rays of light from sunlight into the shade, and to remain there; while negatively heliotropic animals can be compelled to move in the direction of the rays of light, from the shade into direct sunlight, and remain there. A few experiments will better illustrate the nature of helio- 
tropic phenomena than long discussions, and as negatively heliotropic animals are very rare - indeed, much rarer than I formerly assumed-I will illustrate the more simple facts of heliotropism in such an animal and on one which I have had the opportunity of studying in America.

2. The larvæ of Limulus polyphemus - the horseshoe crab - are energetically negatively heliotropic for some time after they have escaped from the egg. If these animals, which live for months without food in a small vessel of sea water, are brought near a window, they collect during the

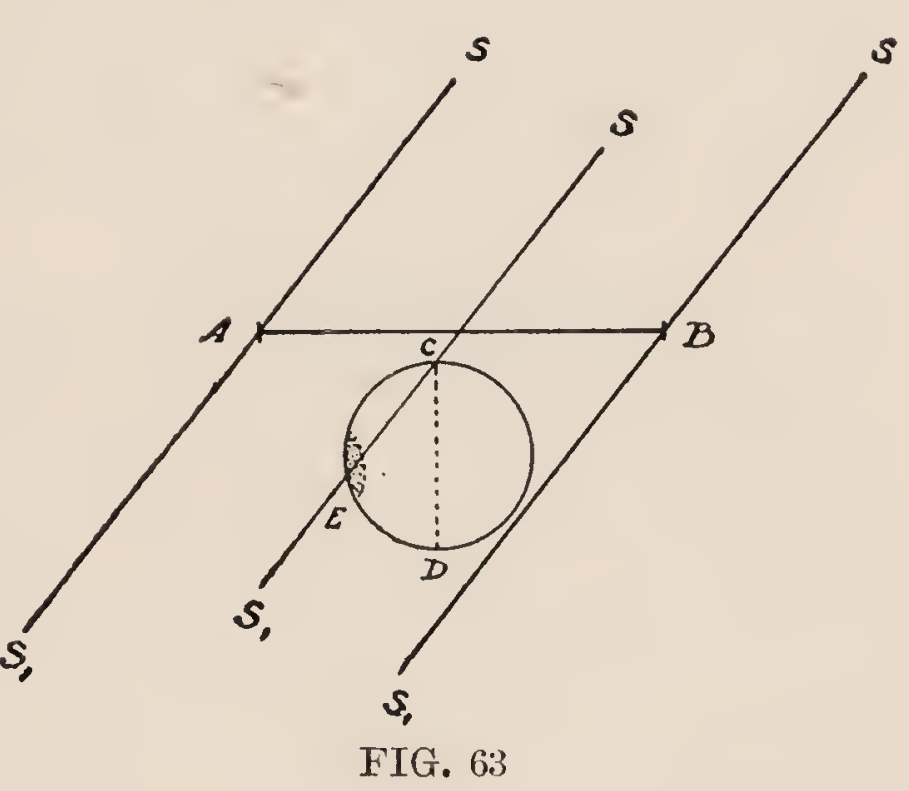
day in a narrow zone on the room side of the vessel. If the vessel is carefully turned through an angle of $180^{\circ}$, so that the animals are brought to the window side, they at once return in perfectly straight lines to the room side of the dish. The animals are clumsy in their walking movements, and tumble over very easily — a fact which must of course be considered.

It can easily be shown that the movements of the animals follow the direction of the rays of light. Let $A B$ in Fig. 63 represent the horizontal section of a window through which direct sunlight falls obliquely. $S S_{1}$ are the horizontal projections of the sun's rays. The circle is the section of the vessel in which the animals are contained. At the beginning of the experiment the larvæ are at $C$. Immediately after being exposed to the light they begin to migrate, not, however, in the direction $C D$, perpendicular to the plane of the window, but in the direction of the sun's rays $S S_{1}$. 
Nor do the animals collect at $D$ on the room side of the dish, but rather at $E$. The movements, therefore, occur in the clirection of the rays of light. If the experiment is to be demonstrated to others, a shadow may be thrown into the vessel by a rod, in which case one can see directly that the

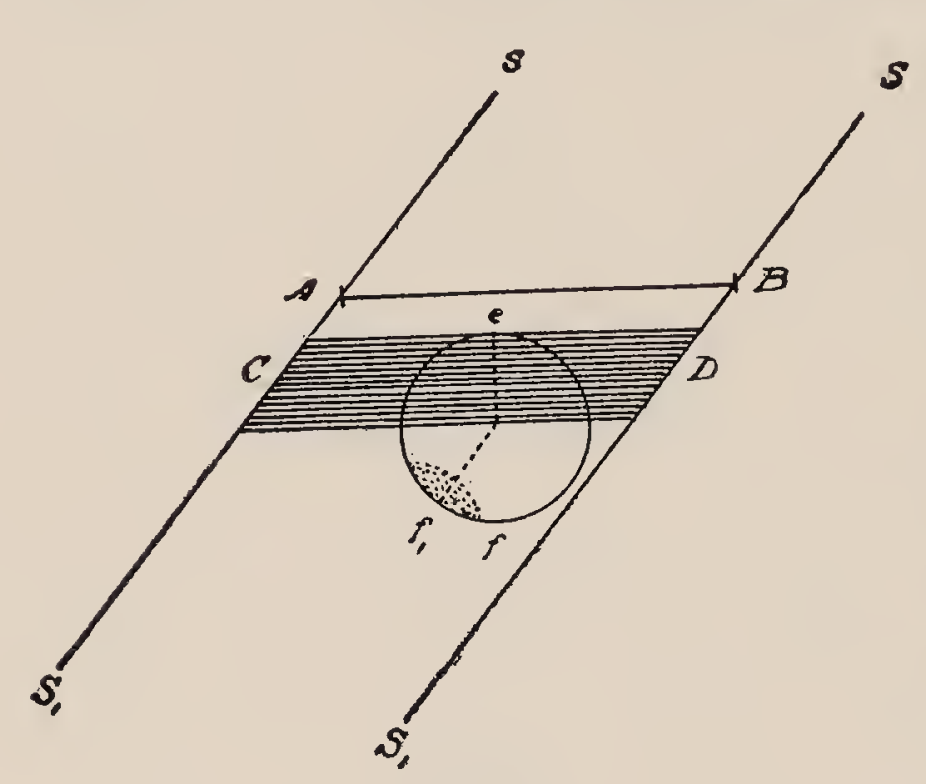

FIG. 64 animals move parallel to the shadow.

Attention need scarcely be called to the fact that if rays of light strike the animal simultaneously from various directions, and the animal is able to move freely in all directions, the more intense rays will determine the direc-

tion of the progressive movements.

That it makes no difference to the negatively heliotropic Limulus larvæ whether they go from regions of less intense light to regions of greater intensity - that is to say, from the "dark" into the "light" - but that only the direction of rays of light determines the direction of the progressive movements, is shown by the following experiment. Let $A B$ in Fig. 64 again be the plane of the window; $S S_{1}$ the horizontal projection of the sun's rays falling into the room obliquely from without and above. The horizontal part of the window frame casts the shadow $C D$ upon the table. The strip $C D$ will, of course, be illuminated by reflected daylight. I placed the vessel ef containing the Limulus larvæ upon the table so that the window side $e$ of the dish lay in the shadow, while the room side $f$ of the dish was in the sunlight. At the beginning of the experiment the larvæ were collected in the shadow on the side of the dish nearest the window. They at once began to move to the room side in the path of 
the dotted line of $f_{1}$. In the shadow the animals were oriented by the diffused light, and as the rays fell into the dish symmetrically from both right and left, the animals at first moved in a line perpendicular to the plane of the window, but as soon as they came out of the shade into the direct sunlight, they did not turn about, nor did they even hesitate, but followed in the direction of the sun's rays to $f_{1}$, where they remained. The animals went thus from the "dark" into the "light."

To overcome the objection that the animals "love the light," I made a third experiment, in which the conditions remained just as in the experiment described above, except that I placed the dish near the window in such a way that the room side was in the shade and the side next the window in direct sunlight. The animals which were on the window side at the beginning of the experiment moved, as before, in the direction of the sun's rays out of the sun into the shade, where they remained.

I wish to emphasize the fact that the animals remained permanently on the room side of the dish, under all conditions, no matter whether this part was in the sunlight, in diffuse daylight, or in twilight.

These facts show, first, that the larvæ of Limulus move in the direction of the rays of light, away from the source of light; and, secondly, that they do so even when by so doing they pass from shade into direct sunlight (or vice versa).

I call those animals which are oriented by light heliotropic, no matter whether, besides this, they execute progressive movements or not. But I wish to point out that not every animal that is sensitive to light is also heliotropic. As we shall see below, aside from the heliotropic, there is another reaction to light, which does not consist in a direct orientation of the animal. 
II. ON THE THEORY OF HELIOTROPISM

Every attempt to formulate a theory of heliotropism is handicapped by our ignorance of the nature of the changes which are produced by the light in the illuminated tissues. If we acknowledge this gap, then the rest of the heliotropic effects of light upon animals may, perhaps, be understood as follows: Let us imagine any number of sections made parallel to the three principal axes of a bilaterally symmetrical, heliotropic animal. Of these elements into which the animal has been divided, always two which occupy symmetrical positions with reference to the median plane of the animal possess equal irritability. Every other two elements, however, possess unequal irritability, and generally the irritability of the oral end is greater than that of the aboral end. Corresponding elements on the dorsal and ventral sides have unequal irritabilities. I imagine the importance of this distribution of irritability for the orientation of the animals to be as follows: If the light strikes one side of the animal, changes occur in the illuminated tissues, which at present are unknown. In consequence, a change occurs in the tension of the muscles (or the contractile elements which act like muscles), which may be of two kinds: the light either brings about an increased tension of the muscles on that side of the animal which is exposed to the light (or of those muscles which turn the animal toward this side); or the opposite occurs, and the light brings about a decrease in the tension of these muscles and a preponderance of the tension of their antagonists. The first takes place, as I assume, in positively heliotropic animals; the second, in negatively heliotropic animals. These assumptions explain the orientation of animals by light. Let $S S_{1}$ (Fig. 65) be parallel rays of light; $a$ the oral, $b$ the aboral end of a heliotropic animal. At the beginning of the experiment the animals move in a straight line in the direction $b a$. The 
tension of the muscles turning the animal to the right and to the left is then the same. As soon, however, as the rays of light $S S_{1}$ strike the right side, the tension of the muscles which turn the animal toward the light side either becomes (1) greater or (2) less; and this difference in the tension of the symmetrically situated muscles will in either case be greater at the more irritable, oral end $a$ of the animal than at the less irritable, aboral end $b$. In the former case the animal will be forced to assume the position $b a_{1}$, and, further more, under the same conditions, to bring its median plane into the direction of the rays of light; it is positively heliotropic. In the latter case it will be forced to assume the position $b a_{2}$; it is negatively heliotropic.

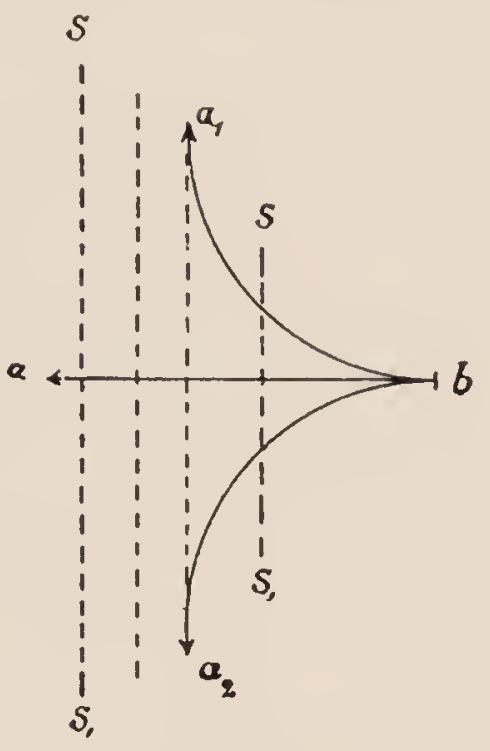

FIG. 65 As soon as the plane of symmetry coincides with the direction of the rays of light, symmetrically situated points on the body of the animal are struck at the same angle by equally strong rays of light, and the animal can then no longer be driven either to the right or to the left by the light, and consequently continues to move in the direction of the rays of light. As soon, however, as the animal is again disturbed in its movements in this direction, through some other external or internal stimulus, symmetrically situated points of the animal are again stimulated unequally by the light. In consequence there is a corresponding change in the tension of the symmetrical muscles, and as a result of this the animal is again brought into its proper orientation.

I wish, however, particularly to emphasize the fact that the progressive movement of heliotropic animals in the direction of the rays of light is a fact which can be directly observed and demonstrated, and is not a mere hypothesis.

The question further arises whether facts are indeed at 
hand to show that the phenomena of the liberation of energy produced by the light show different characteristics in positively and negatively heliotropic animals to correspond with this theory. Negatively as well as positively heliotropic animals execute progressive movements under the influence of light, independently of their orientation, and a difference could be expected only in the efforts which the animal must make to execute the given progressive movements. It might be thought that in positively heliotropic animals light brings about a condition of the muscles or the nervous system in which the liberation of energy is made easier, while in negatively heliotropic animals a condition of the muscles is brought about by the light in which the liberation of energy is made more difficult. I have given some observations in sec. 4 of this paper which seem to sustain such an assumption. Before doing this, however, I wish to acquaint the reader with a series of new facts which deal with the transformation of positive heliotropism into negative, and vice versa.

III. ON THE TRANSFORMATION OF POSITIVE HELIOTROPISM INTO NEGATIVE HELIOTROPISM, AND THE REVERSE

1. In my earlier papers I was able to describe such animals only as were constantly positively or negatively heliotropic. Later, Groom and I described some observations at Naples on the behavior of the nauplii of Balanus perforatus, and certain other marine animals, which were at times negatively heliotropic, and at other times positively heliotropic. ${ }^{1}$ We found that the intensity of the light determines the sense of heliotropism in these animals. Above a certain intensity light makes these animals negatively heliotropic, and this the more quickly the greater the intensity of the light. By lamplight the animals were always positively heliotropic.

I have made further experiments on pelagic animals at

1"Der Heliotropismus der Nauplien von Balanus perforatus," Biologisches Centralblutt, Vol. X. 
Woods Hole on the artificial transformation of positive heliotropism into negative heliotropism, and viceversa. I obtained the best results in the larve of Polygordius in the early stages of development. These appeared in countless numbers for about two weeks in June in the surface dredging near the coast of Woods Hole, and I was able to collect my material in abundance and in good condition.

Immediately after the larvæ had been caught they were always negatively heliotropic. When they were left undisturbed, they became positively heliotropic in the course of several hours. This transformation occurred more rapidly when the vessel was covered than when it was allowed to remain uncovered. If the vessel remained covered permanently, the animals remained positively heliotropic at ordinary room temperatures.

In order to describe more accurately the phenomena which I have observed on the artificial transformation of the sense of heliotropism, I must introduce a new term, namely, the intensity of heliotropism. We find very frequently that in intense light an animal moves exactly in the direction of the rays of light; that in weaker light the direction of the progressive movements in general still follows the direction of the rays of light, but that at any particular moment the median plane of the animal, instead of being exactly parallel to the direction of the rays, may form a slight angle with them. These slight deviations of the median plane from the direction of the rays of light may occur at times toward the right, at times toward the left; and the path of such an animal oscillates continually from the straight line with which we indicate the direction of the rays of light. The amplitude of these oscillations is a function of the intensity of the light, and they become greater when the intensity of the light diminishes. We also find, however, that at the same intensity these oscillations may be unequally great in 
different animals. When other external conditions are the same, we deal in these cases with different degrees or intensities of heliotropic irritability. We therefore measure the intensity of the irritability by the (reciprocal) value of the oscillating deviations of the animal from the direction of the rays of light. It will therefore be understood what is meant when I speak of an increase or a decrease in the positiveness or negativeness of the heliotropism.

2. I succeeded regularly in making the larva of Polygordius negatively heliotropic through an increase of temperature, and positively heliotropic through cooling.

A large number of freshly caught larvæ were distributed into seven glass dishes. Each dish contained thousands of larvæ, and they were all without exception negatively heliotropic. I chose a vessel with such negative animals, and set it into a larger vessel containing ice and salt in order to cool the water containing the animals. The experiment was made before a window facing the north. At the beginning of the experiment, at 2:05 P. M., the temperature in all the vessels was about $16.5^{\circ} \mathrm{C}$. In the course of the next seven minutes the temperature in the dish surrounded by the mixture of ice and salt fell to $11^{\circ} \mathrm{C}$., without a change occurring in the behavior of the animals. No matter how often I changed the orientation of the dish toward the window, the animals went in a straight line back to the room side of the vessel. At 2:15 P. M. the temperature had fallen to $8^{\circ} \mathrm{C}$. A few of the animals then left the negative side of the dish and moved to the window side. The temperature fell to $6^{\circ} \mathrm{C}$., and the larvæ went in swarms to the opposite side. At 2:33 the temperature of the dish was $5^{\circ} \mathrm{C}$, and only a small proportion of the animals were negatively heliotropic. At $2: 30$ at a temperature of $4^{\circ} \mathrm{C}$. only about ten animals remained at the negative side; while the remainder, practically thousands, were collected at the positive side. In the 
other vessels in which the temperature had not been changed, all the animals had remained, without exception, negatively heliotropic. I took pains to keep the temperature in the vessel itself uniform throughout.

Later, when I allowed the temperature in the cooled dish to rise again, the animals gradually became negatively heliotropic as soon as the temperature reached $6^{\circ} \mathrm{C}$. and above.

It could be shown that the absolute height of the temperature, and not the sudden fall in the temperature alone made the animals positively heliotropic. When I removed negatively heliotropic animals from water having a temperature of $23^{\circ} \mathrm{C}$, and brought them suddenly into water having a temperature of $13^{\circ} \mathrm{C}$, they remained negative even when I waited as long as an hour; while, when the temperature sank as low as $7^{\circ} \mathrm{C}$., the animals became positively heliotropic in a few minutes. When the temperature was lower than $6^{\circ} \mathrm{C}$., the animals remained positively heliotropic as long as the temperature remained as low as this (in some experiments this was for two hours). The temperature at which the animals become positive is, of course, not absolutely the same in all experiments. I have repeated the experiments with many modifications, and have always found that when cooled below $+7^{\circ} \mathrm{C}$. all, or almost all, the animals became positively heliotropic.

A few observations on the behavior of these animals at low temperatures may perhaps be of interest. The positiveness of the animals at $+4^{\circ} \mathrm{C}$. was greater than the positiveness of the animals at $+7^{\circ} \mathrm{C}$., the oscillations from the straight line were smaller, and their movements more energetic - a fact which I had not anticipated. I still observed positively heliotropic reactions at a temperature of $+0.4^{\circ} \mathrm{C}$. The reaction to light ceased at $-0.5^{\circ} \mathrm{C}$., although the animals still moved, and at $-2^{\circ} \mathrm{C}$. the animals passed into 
cold rigor. As the animals are exceedingly small, it is reasonable to assume that their temperature was almost identical with that of the sea-water in which they were contained.

Positively heliotropic Polygordius larvæ can easily be made negatively heliotropic through an increase in temperature. I put some animals, which at the room temperature of $+24^{\circ} \mathrm{C}$. had all become positively heliotropic, into a dish, and put the dish, as usual, into another glass vessel of larger size. At 10:45 A. M. warm water was poured into the outer vessel. At 10:52 the temperature of the dish containing the animals was $25.5^{\circ} \mathrm{C}$, and all the animals were still positively heliotropic. Five minutes later, when the temperature had reached $29^{\circ} \mathrm{C}$, all the animals became negatively heliotropic. The reaction continued the same until $34^{\circ} \mathrm{C}$, when the animals no longer reacted to light.

In another experiment the animals were positively heliotropic at the room temperature of $17^{\circ} \mathrm{C}$. When I raised the temperature to $24^{\circ} \mathrm{C}$, in the same way, as before, the animals became negatively heliotropic, and their negativeness increased at first with an increase in temperature. Just as it was possible every time to make negatively heliotropic animals positive through cooling, it was also possible every time to make positively heliotropic animals negative through heating. I have repeated the experiments many times, and besides have demonstrated them to others. After what has been said I need scarcely mention that positively heliotropic animals become more energetically positive through cooling, while negatively heliotropic animals become more energetically negative through warming.

3. The heliotropism of Polygordius larvæ can also be influenced by light. This influence consists chiefly in the fact that direct sunlight makes positively heliotropic animals negative. I did not succeed in making negatively heliotropic 
larve positive by exposing them to weak light. I must emphasize the fact that the larve which I caught during the first week did not become negative in direct sunlight; while the larvæ caught during the second week promptly showed the effect of the sunlight by becoming negative. I do not know what caused this difference. I tried to see now whether the same animals, which I was able to make negatively heliotropic in a few moments at ordinary temperature by direct sunlight, would still be influenced in the same way as before by low temperature. I found that at a temperature below $+y^{\circ} C$. even the strongest sunlight was not able to make the animals negatively heliotropic. (The influence of temperature in this case reminds one of the critical temperature of gases.) It will be necessary to describe these experiments in somewhat greater detail.

Some Polygordius larvæ had stood quietly in the north room for three days, and had become positively heliotropic. I took one portion of the animals and put them into direct sunlight. The temperature of the water in the vessel containing them was $20^{\circ} \mathrm{C}$. In order to keep the sun from heating the water, the vessel containing the animals was set into another large vessel of glass containing sea-water and pieces of ice. The water in the outer vessel was kept in constant circulation by stirring. Even though the temperature in the dish containing the animals, which was exposed to direct sunlight, not only did not rise, but even sank a little - it fell during the experiment to $15^{\circ} \mathrm{C}$. - the animals, nevertheless, became all negatively heliotropic in three minutes under the influence of the direct sunlight. When later I carried the animals back into the north room and kept the temperature constant at $15^{\circ}-16^{\circ} \mathrm{C}$., they again became positively heliotropic in the course of twenty minutes. The sunlight must be very intense in order to bring about this effect. If the dish containing the animals 
is covered with a red or blue glass, the transformation does not take place. I tried to discover now whether at a temperature of $7^{\circ} \mathrm{C}$. and less the sunlight would still be able to make the positive animals negative. I again took some animals which had become positively heliotropic in the north room, and convinced myself first of all that at a constant temperature of $20^{\circ} \mathrm{C}$. they would become negatively heliotropic in direct sunlight in a few minutes. I then returned them to the north room, and here the animals again became positively heliotropic at the same temperature in the course of fifteen minutes. After this I filled the space between the dish containing the animals, and the outer vessel, while still in the north room, with ice and salt, and waited until the temperature in the dish containing the animals had fallen to $8^{\circ} \mathrm{C}$. During this procedure the animals had only become more intensely positively heliotropic. I now brought the entire apparatus into a south room and exposed it to the direct sunlight. The animals which had been disturbed by being carried from room to room at once moved to the window side of the dish when exposed to sunlight. Not one animal became negative or indifferent. The temperature dropped to $5^{\circ} \mathrm{C}$. The animals remained strongly positively heliotropic. I kept the temperature of the water in the vessel containing the animals between $5^{\circ} \mathrm{C}$. and $3^{\circ} \mathrm{C}$. for an hour. Greater differences in temperature in the dish containing the animals were prevented through constant stirring of the fluid in the outer vessel. The animals remained positively heliotropic permanently. I now allowed the temperature of the dish containing the animals to rise rapidly by stopping the addition of ice. In fifteen minutes the temperature reached $7^{\circ} \mathrm{C}$., and a few animals had already become negative at this point. In the next fourteen minutes the temperature rose to $9^{\circ} \mathrm{C}$, and more than half of the animals became negatively heliotropic. Fifteen minutes later, 
at a temperature of $15^{\circ} \mathrm{C}$., the majority of the animals were negatively heliotropic. The sunlight, which had before and later made the majority of the animals negatively heliotropic in a few minutes at a constant temperature of about $15^{\circ} \mathrm{C}$., had no effect upon the same animals when the temperature was $3-$ $7^{\circ} \mathrm{C}$. When I returned the animals to the north room, they all again became positively heliotropic, even though the temperature at the same time rose to $21^{\circ} \mathrm{C}$.

I have repeated these experiments many times, with numerous modifications, but always with essentially the same result.

4. Further experiments showed that through an increase in the concentration of the sea-water the same results can be obtained as through a lowering of the temperature. Negatively heliotropic larve become positively heliotropic, and positively heliotropic larve become still more positive. Through a decrease in the concentration of the sea-water the same effect is obtained as through an increase in its temperature. The positively heliotropic animals become negatively heliotropic, and the negatively heliotropic animals more strongly negative.

I prepared four solutions of a higher concentration than the sea-water by adding chemically pure sodium chloride to the normal sea-water. To each 100 c.c. of sea-water the following amounts of salt were added: $0.6 \mathrm{~g} ., 1 \mathrm{~g}$., $1.3 \mathrm{~g}$., and $1.6 \mathrm{~g}$. In order to avoid differences in temperature $\mathrm{I}$ allowed the solutions, carefully protected from evaporation, to stand for a day in the room in which the animals were kept. Polygordius larvæ which were energetically negatively heliotropic were then distributed into these solutions. The majority remained negatively heliotropic in the weakest solution, but a few of the animals went to the window side. In the next three solutions almost all the animals at once became positively heliotropic. In the most concentrated so- 
lution the animals at first showed no reaction; they fell to the bottom, and remained there as though dead. Later a few recovered, which, without exception, were positively heliotropic.

Animals which were already positively heliotropic in normal sea-water became more energetically so when introduced into concentrated sea-water.

Dilute sea-water made the larvæ negatively heliotropic. To three dishes, each containing 100 c.c. of sea-water, were added 20,40, and 60 c.c., respectively, of fresh water. All the solutions were at room temperature. Positively heliotropic larvæ were distributed in sufficient numbers into these three solutions.

The larvæ remained positive in the first solution of 100 c.c. of sea-water +20 c.c. of fresh water. Only three of the thousands of larvæ which I had introduced into this solution became negatively heliotropic. In the second solution only about half of the animals remained positive, the other half at once becoming negatively heliotropic. In the third solution of 100 c.c. of sea-water +60 c.c. of fresh water the animals lay on the bottom for a few minutes without reacting, and then they slowly recovered, and all crept to the room side of the vessel. Without exception they all became negatively heliotropic. Negatively heliotropic animals when introduced into the diluted sea-water become only more strongly negative.

We must ask the question whether the suddenness of the change in the concentration of the salt solution, or merely the absolute concentration, determines the change in the heliotropism. In answer it must be said that on the same day, and generally also on the next day, there is no change in the behavior of the animals when they remain in the same solutions. But after that changes may occur. It must be kept in mind that the amount of water contained in the tissues of 
an animal is dependent, not only upon the concentration of the salt solution in which it lives, but also upon the amount of water which is lost by secretion. It is therefore clear that in time an adaptation of the animal to the altered concentration of the sea-water may occur. The temperature in all these experiments was usually about $20^{\circ} \mathrm{C}$.

5. I tried to see whether it is possible to make animals positively heliotropic in a diluted salt solution by lowering the temperature. Positively heliotropic larvæ were introduced into sea-water to which 72 per cent. of fresh water, by volume, had been added. At the beginning of the experiment the temperature was $19.5^{\circ} \mathrm{C}$. The animals at once became negatively heliotropic. I then began to lower the temperature. At the same time as a control I subjected a number of negatively heliotropic animals contained in normal sea-water to the same lowering of temperature. When the temperature reached $11^{\circ} \mathrm{C}$, a few of the animals in normal sea-water became positively heliotropic, and at $7^{\circ} \mathrm{C}$. the majority of the animals in normal sea-water became positively heliotropic. In the dilute salt solution, on the other hand, all the animals remained negatively heliotropic. Only they reacted more slowly than the animals kept in the normal sea-water. At $+4^{\circ} \mathrm{C}$. they also remained negatively heliotropic, if they reacted at all; while those kept in the normal sea-water at this temperature were very energetically positively heliotropic. When the temperature again rose, the reactions of the animals in the dilute sea-water again became lively, but they, of course, remained negatively heliotropic. I have mentioned the fact that animals which have been kept several days in dilute sea-water may again become positively heliotropic. In such animals it was also possible by lowering the temperature to make at least a few positively heliotropic.

6 . It is worthy of note that I found the same dependence 
of heliotropism upon the temperature and concentration of the sea-water in marine animals which are far removed in the animal scale from the Annelids, namely, Copepods. I worked on a series of Copepods which were always collected by the tow net, a number of which, however, are not yet classified. The species which was found in largest numbers is, according to Dr. Bumpus, probably Temora longicornis. The freshly caught Copepods were not negatively heliotropic at first like the Polygordius larvæ, but positively heliotropic; the majority of these, however, soon became negatively heliotropic. Jarring made them positively heliotropic temporarily. They were much less resistant than Polygordius larvæ. An increase in temperature made the positively heliotropic Copepods negatively heliotropic, and increased the negativeness of the Copepods already negatively heliotropic. A lowering of the temperature made the negatively heliotropic Copepods positive and increased the positiveness of the positively heliotropic Copepods. An increase in the concentration of the sea-water increased the positiveness, and a decrease in concentration increased the negativeness, of the Copepods. A few examples may serve to illustrate these facts. At $+22^{\circ}$ C. the Copepods were positively heliotropic. In the course of five minutes the temperature was raised to $26^{\circ} \mathrm{C}$, when the majority became negative. I continued to raise the temperature to $32^{\circ} \mathrm{C}$, but the animals remained negative. I then cooled the water. The animals became positive when only $20^{\circ} \mathrm{C}$. was reached, and remained so while the temperature continued to fall. The change in the temperature seems to be the important circumstance in Copepods which changes the sense of heliotropism. In another experiment the Copepods were negative at $24^{\circ} \mathrm{C}$. I cooled the vessel rapidly so that the temperature fell to $21^{\circ} \mathrm{C}$.; at this point a part of the animals already became positively heliotropic. At a temperature of $7^{\circ} \mathrm{C}$. all had 
become positively heliotropic. Later, when the temperature agrain rose, they again became negatively heliotropic

The addition of 80 parts of fresh water to 100 parts of normal sea-water made positively heliotropic Copepods negatively heliotropic; the addition of 60 per cent. of fresh water made a number of them negative, and a smaller addition had little or no effect.

Almost all negative Copepods became positively heliotropic when introduced into sea-water to which $1.5 \mathrm{~g}$. of $\mathrm{NaCl}$ had been added to each 100 c.c. of sea-water. After the detailed description of the behavior of the Polygordius larve these statements may suffice.

7. I have already shown in my earlier papers on heliotropism that there is scarcely a heliotropic reaction in plants which cannot also be demonstrated in animals. This fact is again corroborated by the phenomena which we have described here. I quote the following from Strassburger's well-known investigation on the effect of light and heat upon swarm spores: ${ }^{1}$

When I had [Hæmatococcus] swarm spores before me which had collected, at the ordinary temperature of the room in which I worked $\left(16-18^{\circ} \mathrm{C}\right.$.) at the positive edge of the drop, I was sure of being able to transfer them to the negative side of the drop, at the same light intensity, when I exposed the preparation to a temperature of about $4^{\circ} \mathrm{C}$. At this low temperature almost all of them went to the negative edge of the drop. On the other hand, I was almost as certain to find the most negatively heliotropic Hæmatococcus swarm spores at the positive edge of the drop when I exposed the preparation to a temperature of about $35^{\circ} \mathrm{C}$.

Only in the sign of heliotropism is there a difference in the effect of heat on Hæmatococcus swarm spores and Polygordius larvæ and Copepods. I consider it possible that animals may be found which become negatively heliotropic when cooled. Massart ${ }^{2}$ found similar phenomena in Flagel-

1 Jena, 1878, p. 56.

2 JEAx MAssart, Bulletin de l'Acallémie royale de Belgc, Vol. IXIJ (1891). 
lates. Chromalina approaches the source of light at $20^{\circ}$, but flees from it at $5^{\circ} \mathrm{C}$. The sense of geotropism is also altered in an entirely analogous way, and we shall see in the next section that a similar result is also obtained in Polygordius larvæ.

IV. DIFFERENCES IN THE MANNER OF LOCOMOTION BETWEEN POSITIVELY AND NEGATIVELY HELIOTROPIC ANIMALS

Having seen how certain positively heliotropic animals can be made negatively heliotropic, let us ask whether another difference, independent of the direction, can be discovered in the movement of positively and negatively heliotropic animals. Such a question can, of course, be answered only in animals which can be studied both in the negatively and positively heliotropic condition.

The larvæ of Limulus polyphemus are positively heliotropic immediately after hatching from the egg. Later they are negatively heliotropic. T'he animals can creep as well as swim in all stages of their development. In fact, one can observe the animals executing both forms of movement in every stage of development. In their heliotropic movements there is, however, a typical and constant difference: the positively heliotropic movements are always carried out by swimming, the negatively heliotropic by crawling, motions. The swimming movements are easy and graceful; the walking movements, clumsy. I believed at first that this difference in the manner of movement was dependent chiefly upon the fact that the rays of light fell into the vessel from above and without, and that in consequence the positively heliotropic animals were attracted to the upper surface. I do not believe, however, that this is a complete explanation. I had a chance to convince myself in the larvæ of Polygordius that this difference in the manner of movement between positively and negatively heliotropic animals can exist inde- 
pendently of the light. Polygordius larve usually swim at the surface of the water when positive, while in their negatively heliotropic condition they usually creep along the bottom. When I introduced a large number of Polygordius larre into a eudiometer tube filled with sea-water, and set it in a vertical position in a dark room, the larvæ were not distributed equally in the tube after some time-in about twenty-four hours - but one collection was usually found at the surface of the water, and a second at the bottom. If the tube was now carefully and suddenly brought to the light, all the larve at the surface layer showed themselves to be positively heliotropic, while all the larvæ at the bottom were negatively heliotropic. It could further be easily shown that positively heliotropic Polygordius larvæ went to the surface of the tube when put into the dark room, while negatively heliotropic Polygordius larvæ went to the bottom. Further, positively heliotropic Polygordius larvæ may, as has already been mentioned, be made negatively heliotropic by warming. When a eudiometer tube containing Polygordius larve at its surface was warmed in a dark room, the larve went to the bottom of the tube. When the tube was cooled in the dark room, below $7^{\circ} \mathrm{C}$, all the larvæ left the bottom and collected at the surface. Both the ascent and the descent of the larvæ are brought about by active swimming motions. It seems to me probable that the animal is not only heliotropic, but also geotropic, and that the sense of geotropism is always changed under the samie conditions as the sense of heliotropism. Negative geotropism is associated with positive heliotropism, and positive geotropism with negative heliotropism. Nevertheless, this difference persists, that the positively heliotropic animals (which at the same time are negatively geotropic) always swim; while the negatively heliotropic animals (which are also positively geotropic) lie or creep upon the bottom, and, it seems to me, 
also swim less easily. It is, therefore, very possible that a more favorable condition for the liberation of energy accompanies positive heliotropism, while a more difficult condition accompanies negative heliotropism.

Finally, I shall mention a circumstance which possibly belongs to the same group of phenomena, and which first led me to investigate the effects of light on animals. During hard glacier trips I noticed that the fatigue which set in disappeared at once when I removed my snow spectacles and exposed my eyes to the full light. On the other hand, it is well known that the intense light of the snow-fields increases the fatigue when one is exposed to it for a long time. Light certainly has something to do with the liberation of energy, either facilitating it or rendering it more difficult; and it seems that in certain organisms it may call forth both kinds of effects under different circumstances. Whether these observations have any deeper significance or not is to be determined by further experiments.

\section{HELIOTROPIC AND PHOTOKINETIC ANIMALS}

1. Huxley states in one of his essays that plants must have a nervous system, because Darwin observed reactions in Drosera which in some points are similar to those in animals. In view of the identity of heliotropism in animals and plants, the same reasoning would force us to assume that plants possess eyes. The only conclusion, however, which may safely be drawn from these facts is that the eyes owe their significance for sight, among other things, to a condition which is found also in the skin of many animals and in plants; namely, elements which undergo certain, but at present unknown, changes through light. It is not even necessary that these elements be everywhere entirely identical, either physically, chemically, or morphologically. As is well known, certain elements are present in our retina 
which move under the influence of light. If heliotropism exists in this case, it is possible that its sense varies. The difficulties which confront one in investigating this problem are greater than in the case of the Polygordius larvæ. Even though one assumes that certain elements of the retina are heliotropic, nevertheless one of the important conditions in our sight-namely, the perception of differences in intensity - cannot possibly be attributed to heliotropic reactions; for we saw that heliotropic animals moved not only out of the dark into the light, but also in the reverse direction, if only the direction of the orienting rays remained the same. It is possible that the perception of differences in the intensity of light by our eyes depends upon specific elements of the retina which react especially to changes in the intensity of the rays of light, Be this as it may, there are certain animals which are not, or at least not very markedly, oriented by the rays of light; which are, therefore, not outspokenly heliotropic, but which react very promptly to differences or, more correctly, to changes in the intensity of the light. These I will term photokinetic ${ }^{1}$ animals. A species of the fresh-water Planarian, for which I am indebted to Dr. Wheeler, is photokinetic. If the animals are put into a large dish of water, they creep about in every direction. They are not oriented by the light. Yet one observes a difference in the behavior of the animals, depending upon whether they move from regions of more intense light to regions of less intensity, or the reverse. A decrease in the intensity of the light tends to make the animals come to rest, while an increase in the intensity increases their tendency to move. It thus happens that these animals gradually collect in such places in the dish where the intensity of the light is a relative minimum. This phenomenon, and at the same time the difference in the behavior of such animals 
from the behavior of heliotropic animals, are best shown in the following experiment: $A B$ (Fig. 66) is the plane of the window; $a b c$, the vessel containing the animals. At one point $b c$ the outer surface of the vessel is made impermeable to rays of light by means of black paper. It can easily be

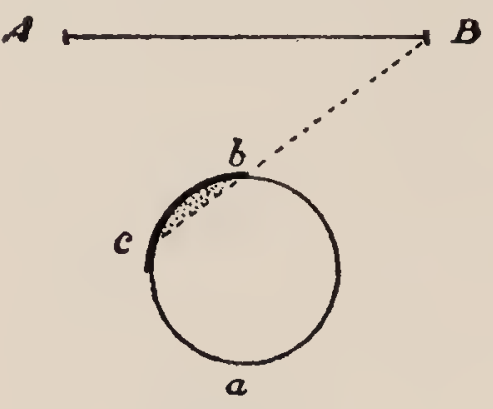

FIG. 66 seen that at $b c$ a small section of the circle is not struck by the rays of light falling upon it from without. If the Planaria at the beginning of the experiment are brought to the window side of the vessel, but so that they are not struck by the light, all, or almost all, the animals are found in a few hours, or on the following day, under the opaque paper $b c$ where the intensity of the illumination is least. If the same experiment is made with negatively heliotropic Limulus larvæ, the larvæ move to the room side $a$ of the vessel, and remain there permanently. It is clear, under these circumstances, that when these Planariæ are left quietly for some days in a cylindrical vessel abcd (Fig. 67), all the animals finally collect at the two sides $c$ and $d$, as was observed by Dr. Wheeler. Heliotropic animals in the same vessel either go immediately to the window side $a$ or the room side $b$ of the vessel, and remain there.

This mode of reaction to changes in the intensity of light occurs probably also in angleworms; perhaps, too, in other animals. It is, moreover, pos-

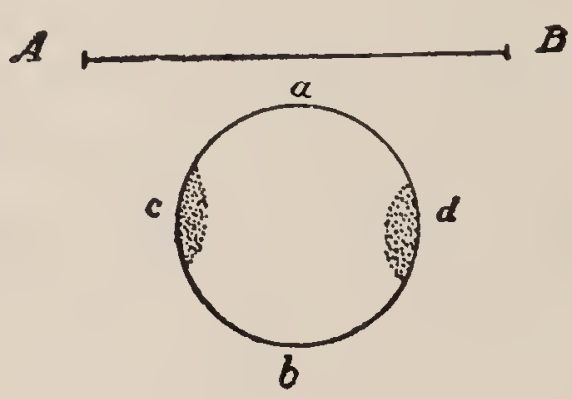

FIG. 67 sible that heliotropism and photokinesis are associated in certain animals ${ }^{1}$ - a subject which I still wish to investigate. ${ }^{2}$

1E. g., Spirographis spallanzanii.

2 I had previously noticed that in some animals, which $I$ at that time considered negatively heliotropic, the typical heliotropic experiment did not succeed very well. $I$ attributed this to secondary circumstances. I now consider it possible, however, that the experiments which I described, for example those on the larvæ of beetles, indicate as much the existence of photokinesis as negative heliotropism. I shall make further experiments in this direction. 
2. There are photokinetic animals which react more rapidly to changes in the intensity of the light than do Planarians. I noticed this form of reaction at Naples in certain Annelids living in tubes; for example, Serpula uncinata. The gills of the animals are often exposed to the light. When the hand is moved between the animals and the source of light, they quickly draw back into their tubes as soon as they are struck by the shadow. In order to see whether positive and negative changes in the intensity of the light had the same effect, I made the following experiment: A glass aquarium which was closed by a glass cover was set upon an isolated table about $2 \mathrm{~m}$. distant from the window. When I closed the shutters rapidly, the worms quickly withdrew into their tubes, much as does a snail when touched suddenly. The shutters did not close absolutely, and it was always light enough in the room to observe the animals. After some time the animals would again stretch out their gills. When I now suddenly opened the shutters quickly, the animals did not react. Even when the animals had withdrawn into their tubes, an increase in the intensity of the light did not again bring them out. It is therefore only the decrease in the intensity of the light which acts as a stimulus upon the animals. One notices, however, that these reactions cannot always be relied upon. Andrews has noticed such reactions also in Annelids whose gills are free from eyes or eye-like organs. ${ }^{1}$

VI. ON SOME PHYSIOLOGICAL CONDITIONS WHICH DETERMINE THE DEPTH-DISTRIBUTION AND DEPTH-MIGRATION OF MARINE ANIMALS

1. Investigations concerning the depth-distribution of marine animals seem to show that we meet with a considerable amount of animal life only in two regions of the sea-

1E. A. Axprews, Journal of Morphology, Vol. V (1891). 
at the surface down to a depth of perhaps $400 \mathrm{~m}$. and at the bottom of the sea. Some of the surface, or pelagic, animals show a periodical depth-migration. They come to the surface at night and move down during the daytime. In the Mediterranean, Chun found another migration of a greater period. Animals which always come to the surface in winter, or at least during certain hours of the day, live at greater depths in summer.

The first experimental investigations on the cause of these depth-migrations were made by Groom and myself, and led to the conclusion that in the Nauplii of Balanus perforatus heliotropism alone suffices to account for the fact that they rise to the surface at night and move down during the day. $\quad$ These animals are positively heliotropic to weak light, but strong light soon makes them negatively heliotropic. They are, in consequence, driven into the depths during the day from the surface of the water. They can, however, not go very deep, as the intensity of the light decreases with an increase in the depth of the illuminated layer of water, and becomes so weak at a certain depth that the Nauplii again become positively heliotropic. They must, in consequence, again move toward the surface. As soon, however, as they again come into more intense light, they become negative again. It can therefore be easily seen why these animals do not go to the bottom of the ocean during the day, but are forced to remain in a layer of water which is not too far below the surface. When the light becomes weaker toward evening and in the night, the Nauplii are again forced to move to the surface of the water in consequence of their positive heliotropism.

2. The question may now be asked whether all animals which are found at the surface of the sea are constantly, or at least under certain conditions, positively heliotropic. I 
have made experiments on the small animals obtained in the surface dredgings at Woods Hole, on Copepods, and on the larve of crustaceans, worms, and molluscs, and have thus for. been able to find no pelagic animal of these classes which is not either permanently, or at least at times, positively heliotropic. ${ }^{1}$

3. It would be incorrect, however, to assume that heliotropism is the only condition for the depth-distribution of animals. Just as in the vegetable kingdom positive heliotropism and negative geotropism often act together toward the same end, we must expect similar conditions in the animal kingdom. In a paper on geotropism I have already shown that certain starfish and Actinians, which always live near the surface of the water, are compelled to creep constantly upward, owing to a peculiar form of irritability, and I have made it probable that this irritability is negative geotropism. ${ }^{2}$ I have since been able to convince myself that in certain animals which would be forced by their positive heliotropism alone to go to the surface, other conditions are at work which co-operate with heliotropism. This is the case, for example, in the freshly hatched larvæ of Loligo. These animals are constantly positively heliotropic, and, besides, live at the surface of the sea. When these animals are introduced into a long eudiometer tube filled with seawater which is set up vertically, they move to the surface of the sea-water. As long as the effective rays of light fall into the tube from above, the positive heliotropism of the larvæ would compel them to move upward. I found, however, that the animals come to the surface of the water also in the dark room. Moreover, when the eudiometer tube is exposed to the light, with the upper part of the tube covered

1 I have even found a young pelagic fish at Woods Hole which is as pronouncedly positively heliotropic as the insect larvæ described in a preceding paper. I was not able to ascertain the species to which it belonged. [1903]

2 Part I, p. 176. 
with a cap impermeable to light, the animals which are very energetically positively heliotropic should not go under the dark cap. The latter does, however, actually occur. Hence another and more powerful circumstance is at work besides the positive heliotropism, and this might be negative geot-

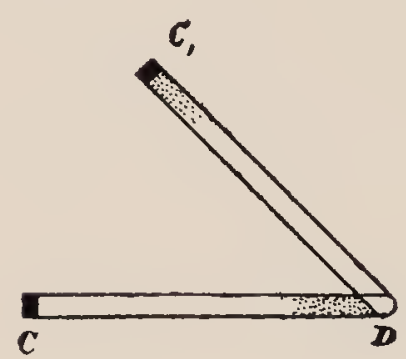

FIG. 68

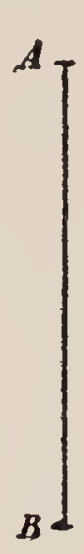

ropism. The temperature in the tube was everywhere the same in these experiments. The need for oxygen does not compel the animals to come to the top in these experiments, for when the eudiometer tube is filled entirely with water, and the open end is turned downward into

a larger vessel filled with water, the animals nevertheless move from this vessel to the cap of the eudiometer, and remain here even though fresh oxygen can reach the animals at this point only through diffusion from below. The significance of this negative geotropism (which I believe it to be) for the upward movement of the Loligo larvæ is shown still more beautifully in the following experiment: $A B$ (Fig. 68) represents a vertical section through the plane of the window; $C D$ is a eudiometer tube filled with sea-water and closed with a cork. If the larvæ at the beginning of the experiment are collected at the room side $C$ of the tube, they all move to the window side $D$ in consequence of their positive heliotropism, and remain there. If, on the other hand, the tube is so placed that it is at an angle with the horizontal - for example, in the position $C_{1} D$ - the larvæ gradually but steadily rise by active swimming movements away from the window to the elevated end $C_{1}$ and remain there. They therefore leave the window side and go to the room side, in spite of their positive heliotropism. To accomplish this the angle $C_{1} D C$ must in this case be not smaller than about $20^{\circ}$.

1 The papers of Wolfgang Ostwald seem, however, to indicate the possibility of a purely physical explanation of this experiment. [1903] 
Of course, negative geotropism is not so closely linked with positive heliotropism in all pelagic animals. If, for example, the Copepods mentioned in this paper are introduced into the eudiometer tube, and the upper end is covered with the opaque capsule, the animals also rise in the tube when they are positively heliotropic. They do not, however, go under the opaque cap, as do the Loligo larvæ, but remain at the highest uncovered portion of the tube. They therefore move upward entirely, or at least mainly, through their positive heliotropism.

4. The effect of temperature upon the depth-migration and depth-distribution of marine animals must yet be studied experimentally. We saw that the larvæ of Polygordius are negatively heliotropic and positively geotropic at high temperatures, but become positively heliotropic and negatively geotropic at lower temperatures. The same transformation of heliotropism occurs in Copepods. It can scarcely be assumed that other animals will not show the same phenomenon. In water whose surface reaches a very high temperature in summer such animals must disappear during that period from the surface, as the high temperature makes them negatively heliotropic, and perhaps also positively geotropic. The negative heliotropism and positive geotropism drive these animals into the depths. As soon, however, as they reach cooler layers of water below the surface, they again become positively heliotropic and negatively geotropic; they must then rise again until they have reached the warmer layers of water. Here they soon again become negatively heliotropic, and perhaps positively geotropic, when they must again sink; and so on. In this way such animals are kept floating at a certain distance under the surface of the water during the summer. When, however, the temperature becomes sufficiently low in the winter, the animals may rise to the surface without becoming negatively heliotropic or positively geotropic. 
Another condition must, however, be taken into consideration, which may have the effect that animals which are constantly positively heliotropic must leave the surface of the water at higher temperatures. As is well known, the processes of oxidation, and consequently the demand for oxygen, rise considerably with an increase in temperature. It is natural that when the demand for oxygen exceeds the supply, the animal can execute no, or only weak, swimming motions, and in consequence falls to the bottom. At any rate, the latter can, indeed, be observed at high temperatures. Loligo larvæ which hold themselves at the surface by swimming motions sink passively as soon as the temperature exceeds $30^{\circ} \mathrm{C}$.

In conclusion I wish to add that I made experiments on most of the animals mentioned in this paper with colored light, and found a universal confirmation of the fact, which I discovered before, that the more strongly refrangible rays of the visible spectrum are the most active heliotropically, as in the case of plants. 


\section{IX}

\section{ON THE DEVELOPMENT OF FISH EMBRYOS WITH SUPPRESSED CIRCULATION ${ }^{1}$}

1. One of the methods which may lead us deeper into the physiology of development consists in removing one link in the chain of such processes in order to see how the further development is influenced by such a step. I recently came upon such an experiment, which I wish to detail in the following pages. The experiment consisted in preventing, by a specific cardiac poison, the beat of the heart and the circulation of the blood in an embryo. I had, indeed, expected that under such circumstances the embryo would not die immediately, but I did expect that its further development would certainly be impossible. In this, however, I was mistaken. Development went on in spite of the elimination of the activity of the heart; in some cases as long as four days - which was nearly half, or one-third, of the duration of the embryonic stage. The consequences of the elimination of the activity of the heart are also in some ways different from what one might expect. The observations have not been completed on all points because of the lateness of the season and lack of material, but I intend to fill in these gaps next season.

The experiments were made upon a marine fish, Fundulus, which is very common at Woods Hole. The eggs were fertilized artificially in normal sea-water. In order to prevent the action of the heart and the circulation in the developing embryo, the eggs were put, half an hour after fertilization, into sea-water to which a sufficient amount of potassium chloride had been added. Potassium chloride can 
be called a specific cardiac poison only in so far as the heart can be brought to a standstill with a much smaller dose than is required to poison the remaining organs of the embryo.

2. At a temperature of $20^{\circ} \mathrm{C}$., and with a plentiful supply of oxygen, the embryos of Fundulus develop in about twelve to fourteen days in normal sea-water. Under these conditions the heart begins to beat about sixty to seventy hours after fertilization. If Fundulus embryos four to six days old are placed in sea-water to which $1.5 \mathrm{~g}$. of $\mathrm{KCl}$ have been added to each 100 c.c. of sea-water, the heart ceases to beat, and death ensues in about one hour, at the outside, in all the embryos. That the poisonous effect of the potassium is not limited to the heart is shown by the fact that the embryo becomes exceedingly restless before the heart ceases to beat. An entirely different series of phenomena ensues when the Fundulus eggs are introduced into the same salt solution about one-half hour after fertilization. The eggs develop in an entirely normal way, the embryos live until the fifth or sixth day, and the cramp-like movements do not set in. Furthermore, I noticed in a few of these embryos very weak and very slow pulsations of the sinus venosus on the third or fourth day.

This activity of the heart did not, however, appear in all the embryos, and when it did appear it did not last long. In no case, however, was the beat of the heart sufficient to cause a circulation of the blood. The circulation in the yolk-sac of the Fundulus embryo can be demonstrated more clearly and easily than the circulation in any of the ordinary preparations used for this purpose.

In the normal embryo the circulation is very marked, even some seventy-five hours after fertilization; but it does not matter how long one waits in the case of the embryos poisoned by adding $1.5 \mathrm{~g}$. of $\mathrm{KCl}$ to each $100 \mathrm{c}$.c. of sea-water-never did I succeed in discovering even the slightest indication of 
a circulation of the blood in the vessels. In spite of this fact, a complete circulatory system was developed which did not differ markelly, in regard to the direction and the branching of the vessels in the embryo and in the yolk-sac, from that of a Fundulus embryo developed in normal seawater. Heaps of red-blood corpuscles were found in the large blood-ressels, such as the arteries of the yolk at the point where they leave the embryo.

The important result of these observations is therefore the fact that a complete vascular system, which is probably identical in its main distribution and in its two vascular divisions with that found in the normal embryo, can be formed without a circulation, and therefore without bloodpressure. A difference between the two, which also determines the limit of the possible identity of the vascular systems in the normal and abnormal embryos, is found in this, that the lumina of the vessels in the poisoned embryo are exceedingly irregular. The lumen of a vessel is in extreme cases rosary-like, narrow and wide spots alternating with each other. This is due to the absence of a sufficient intravascular pressure.

3. It might have been possible that a circulation lasting only a short time had been present which I did not discover. I therefore made experiments with much stronger $\mathrm{KCl}$ solutions - such in which not even a trace of cardiac activity ever appeared. In extreme cases I addded $5 \mathrm{~g}$. of $\mathrm{KCl}$ to 100 c.c. of sea-water. I had previously ascertained that a four-day-old embryo which had developed in normal sea-water dies in two minutes in a 3 per cent. $\mathrm{KCl}$ solution. Nevertheless, the fertilized eggs developed normally in a 5 per cent. $\mathrm{KCl}$ solution. They developed for three to six days, and formed - what is of interest to us here - a heart and a typical vascular system in the embryo and yolk-sac. On the other hand, the development of the embryo as a 
whole was markedly retarded in this concentrated salt solution, so that a definite judgment of the vascular system could be made only so far as the main stems and their branches were concerned. These main stems corresponded with the main stems of the normal embryo. Yet I was never able to discover even the slightest evidence of a heart-beat, much less a circulation. The lack of hydrostatic pressure within the vessels was particularly evident here from the irregularity in the diameter of the blood-vessels; nevertheless, a large number of branches, which gradually decreased in caliber, sprang from the main vessels. In this case, therefore, it is unquestionably true that the process of branching and the growth of the blood-vessels are independent of blood-pressure.

4. The experiments with weak $\mathrm{KCl}$ solutions also deserve mention. In a series of experiments I added 0.25 to $0.5 \mathrm{~g}$. to 100 c.c. of sea-water; normal development occurred in these solutions. The heart-beat and the circulation developed apparently normally. The control eggs, which had been taken from the same culture, but raised in normal sea-water, completed their development in twelve to sixteen days, when the embryos hatched. They lived some four to six weeks after escaping from the egg. In the two $\mathrm{KCl}$ solutions, however, but one embryo, which lived for a day, hatched on the twelfth day in the 0.5 per cent. $\mathrm{KCl}$ solution. All the remaining embryos died between the twelfth and sixteenth day. Death unquestionably resulted from a poisoning of the heart, and not from a general intoxication.

5. The experiments cited above show that a $\mathrm{KCl}$ solution of a definite constitution is the more poisonous the older the embryo. One might think that the chemical constitution of the individual elements of the heart changes with development; but how can we harmonize with this the fact, which has been mentioned above, that the heart of a four-to-five- 
days-old embryo comes to a standstill, and the embryo dies immediately (that is, in less than an hour), in a 1.5 per cent. $\mathrm{KCl}$ solution, while an embryo of the same age which has been kept in this solution from the beginning continues to live in it, and may even show slight evidences of a heartbeat? To say that the embryo adapts itself or becomes accustomed to the poison gives us no new view of the question. Might it not be possible that the $\mathrm{KCl}$ is the more poisonous the greater the work done by the heart in the unit of time, and in consequence the greater the chemical changes going on in it?

According to this, it would be intelligible why a normal embryo, when put into a 1.5 per cent. $\mathrm{KCl}$ solution, dies within a short time, while an equally old embryo which has grown up in the poisonous solution is alive at the same time, and can even show evidences of a heart-beat. The heart of the former beats strongly, while that of the latter works only faintly - so faintly, indeed, that the blood does not even circulate. The embryo can live in a 0.5 per cent. $\mathrm{KCl}$ solution as long as no great demand is made upon the activity of the heart. As soon as the heart begins to beat more strongly at the time of maturity, the embryo dies. This relation of the toxicity of the potassium to the development of energy in the protoplasm, or rather to the chemical changes determining this development of energy, would hold not only for the heart, but also for all the other tissues. The entire question could be decided experimentally, if this has not already been done.

6. All the remaining organs, especially the brain, eyes, ears, and mesoblastic somites develop in the Fundulus embryo without a circulation, without apparent anomalies. Only in one place, where no one has thus far suspected it, did a dependence on the circulation show itself in an unexpected wayin the marking of the yolk-sac, and possibly (but I wish to 
make further experiments in this direction) also of the embryo. The yolk-sac of the Fundulus embryo has a very characteristic tiger-like marking in the second week. Numerous chromatophores, which contain in part black, in part reddish-brown, pigment, develop on the surface of the yolksac of Fundulus. In the early stages of development, on the third day, no definite relation can be discovered between the circulatory system and the chromatophores. The chromatophores are scattered about irregularly upon the blood-vessels, and in the spaces between them. As soon as the circulation is established, however, the chromatophores begin to creep upon the vessels, and in the latter periods of the development, from the tenth day on, the chromatophores are no longer found in the spaces between the vessels, but have all crept upon them. But that is not all. The chromatophores of the yolk-sac of Fundulus have the characteristic amoboid appearance as long as they lie in the spaces between the vessels. Their diameter in any direction is greater than the diameter of an average-sized blood-vessel, and much larger than that of the capillaries. As soon as a chromatophore has reached a blood-vessel, however, it accommodates its entire mass to the surface of the bloodvessel, so that it finally loses its amœboid appearance and apparently forms only a layer about the blood-vessel. The chromatophore cannot leave the surface of the blood-vessel after it has once reached it. This relation is most apparent where a blood-vessel branches. The chromatophore then branches in the same way as the blood-vessel. If the circulation of the blood is prevented by the addition of $\mathrm{KCl}$ to the sea-water, the chromatophores and the blood-vessels both develop, but the chromatophores do not creep upon the blood-vessels. The tiger-like marking of the embryosac of Fundulus is apparently, therefore, a function of the circulation, in so far as the chromatophores are compelled to 
spread orer the surface of the vessels, in consequence perhaps of a chemical stimulus. Whatever may be the cause which compels the chromatophores to creep upon the bloodvessels, my observations certainly show that the distribution of the chromatophores, and therefore the marking of the yolk-sac, is dependent upon the arrangement of the bloodvessels. I will not enter upon this point in greater detail here, as a separate paper on this subject will appear in the Journal of Morphology. I wish only to point out that this is the first case, to my knowledge, in which the physiological explanation of the marking of an animal organ has been found.

7. Nowhere will the mystic find a richer field of unexplainable purposefulness than in the developmental history of the higher animals. In these everything apparently comes into being at the right time and at the right spot, as though each element knew what rôle it had to play in the whole. The heart also begins to beat, apparently, just at the right moment; and I always had the idea-and others will perhaps have shared it - that if the activity of the heart were interfered with, development would soon cease. Our experiments, however, show that, if we do not consider the extreme cases, the development of an embryo in a $\mathrm{KCl}$ solution can keep on normally for three days after the formation of the heart, even though no circulation is established. This latitude for the time of the beginning of the heart-beat is, when compared with the total time of development, very far from the precision expected of a clock-work.

8. In conclusion I wish to emphasize what seems to have been definitely established by these experiments, and what is yet to be determined by further experiments. I consider it certain that the origin, the pathway, and the branching of at least the larger blood-vessels are independent of the blood-pressure. For this reason it is possible for a vascular 
system, which is identical with the vascular system of the normal circulation in the points mentioned, to develop even in the absence of a circulation. The mechanical causes for the growth of the vessel-walls are, therefore, not to be sought inside the vascular lumen, but in all or in single cells of the vessel-wall. The giving off of branches is determined by internal causes acting within the cells of the vessel-wall, or through stimuli arising in their neighborhood which affect these walls as external stimuli affect the formation of stolons in Hydroids. It is possible, however, that the angles at which the branches arise from the main vessels do not correspond absolutely with the angles found in normal embryos. This point still remains to be investigated. Another question which I leave open is whether the circulatory system which is formed in the absence of a circulation is closed or not; that is, whether the capillary branchings of the arteries of the yolk pass over into the capillary branches of the veins. Mere morphological study speaks in favor of this idea, but to settle this point definitely further experiments must be made. I consider it as certain that the tiger-like marking of the yolk-sac of Fundulus is dependent upon the vascular system. 


\section{X}

ON A SIMPLE METHOD OF PRODUCING FROM ONE EGG TWO OR MORE EMBRYOS WHICH ARE GROWN TOGETHER ${ }^{1}$

1. In the effort to extend my work on heteromorphosis to the embryo, I have discovered a simple method of producing at will from a single egg two or more embryos which are grown together. My experiments were made on seaurchins, but it is possible that they can be made with just as great certainty on every other holoblastic egg. Ten minutes after having been artificially fertilized in normal sea-water, eggs of Arbacia were introduced into sea-water to which 100 per cent. of its volume of distilled water had been added. The eggs absorbed so much water in the diluted seawater that their membranes burst and part of their protoplasm flowed out. The eggs then consisted of two connected spheres of protoplasm ( $P$ and $P_{1}$, Fig. 69), as the extruded drop of protoplasm in consequence of its surface tension assumes a spherical form, as does the protoplasm remaining behind inside the membrane. As segmentation has not yet begun at this time, only one of the two droplets contained a nucleus (Fig. 69). When after some time I returned these eggs into normal sea-water, each of the two spheres of protoplasm developed into an entirely normal and complete embryo.

In many cases the two embryos remained connected. More often, however, one of the embryos went to pieces in the course of its early development (in about the morula or blastula stage); and finally many double embryos were gradually separated from each other, in consequence of their

1 Pflügers Archiv, Vol. LV (1894), p. 525. 
active movements in the blastula and gastrula stage. These latter isolated embryos continued their development normally. In this way either separate or "Siamese" twins were formed from a single egg. It often happened that a repeated outflow of the protoplasm occurred, and then three or even

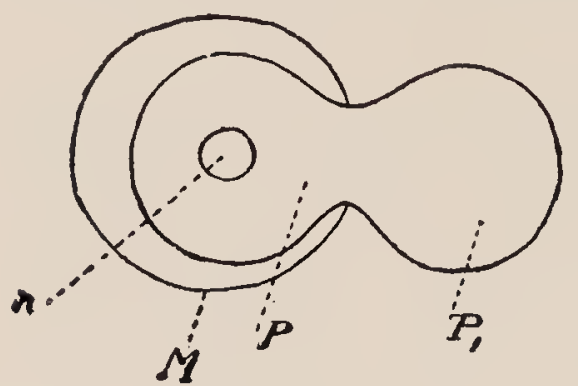

FIG. 69 a larger number of joined protoplasmic drops were formed from one egg. In a number of cases, which was by no means small, I obtained, in consequence, joined triplets or quadruplets. ${ }^{1}$ I suspect, however, that in the gastrula stage many of these multiple embryos are separated from each other through the active movements of the egg, as triplets are relatively rare in the pluteus stage. On the other hand, it was a simple matter to obtain double plutei in large numbers. All these double and triple plutei lived as long (about two weeks), and were as well and as complete in their form, as the plutei produced from a normal egg.

2. As has already been mentioned, the eggs were introduced into the diluted sea-water before the beginning of cleavage, and as only one nucleus was present at this time, only one of the two drops of protoplasm contained a nucleus. Nevertheless, both drops developed into a complete embryo. How did the drop of protoplasm which was at first without a nucleus obtain a nucleus? This happened in a very simple way in the course of cleavage. Cleavage did not take place in the sea-water which had been diluted 100 per cent., but as soon as the eggs were returned to the normal sea-water, cleavage began. The first line of cleavage was perpendicular to the common diameter of both spheres (Fig. 70). The

1 These facts have been questioned by one author on the basis of inadequate and imperfect experiments made by him. During my experiments on artificial parthogenesis I have had a chance to verify amply the statements made in this paper. 
cleavage sphere then developed in the normal way (Fig. 71), and finally a cleavage plane appeared in the extraovate (Fig. 72).

In this way the nucleus becomes distributed through the egg. The further development is simple. The external form of the double sphere is maintained, while both parts divide into smaller cells; each of the two spheres forms a separate blastula cavity, which may communicate in certain cases, although they do not do this as a rule. The spheres

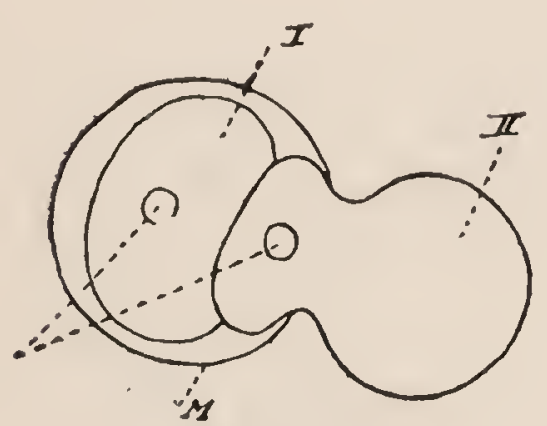

FIG. 70

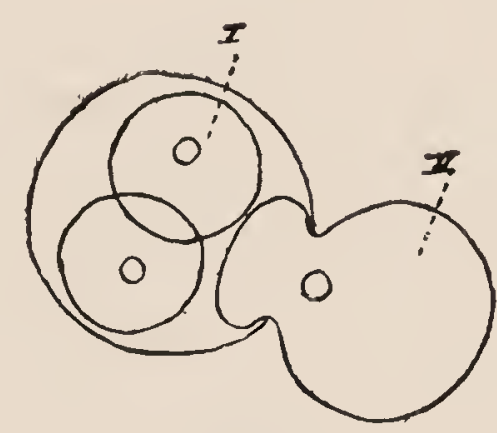

FIG. 71

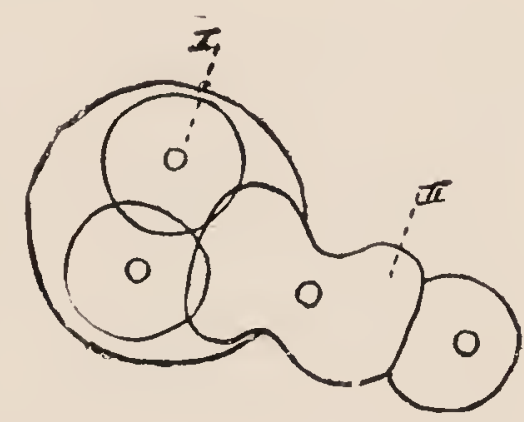

then continue to develop into gastrulæ and plutei. I wish to emphasize especially that both embryos develop from the beginning as entire morulæ and blastulæ, and that no halfformation of any sort appears. In other words, the development goes on as if two independent eggs had been laid side by side, or had been glued together, and each had cleaved and developed entirely independently of the other. The protoplasmic connection of the two double embryos acts, however, differently from the way the gluing together of two eggs would do, as is evidenced by the deformities in the skeletal parts of the two plutei.

3. I have repeated these experiments with eggs in various stages of cleavage. Under these circumstances, also, the protoplasm always flows out in such a way that the cells remain joined together and double spheres are formed. I always obtained the same results, namely, double or multiple embryo.s. Only in the eggs which had developed very far- 
for example, such as were ruptured in the sixty-four-cell stage-did I usually obtain something different, namely, abnormally formed skeletons. Nevertheless at times I obtained under these conditions also real "Siamese" twins.

4. So far as the theoretical importance of these experiments is concerned, the following seems certain: As the bursting of the membranes and the flowing out of the protoplasm is a purely mechanical process determined by osmotic forces, no reason is at hand for assuming that qualitatively or quantitatively the same constituents leave the egg in each case; according to direct observation, the opposite seems to occur with much more certainty. Nevertheless, the extraovate develops into a complete embryo. It follows from this that every part of the protoplasm can form an embryo. So far as the nucleus is concerned, the two spheres obtain entirely different constituents of the nuclear substance. Nevertheless, they develop in exactly the same way to similar embryos, as Driesch has already shown by other methods. Thirdly, my experiments show that the number of embryos which can arise from an egg is determined by the geometrical form which we give the protoplasm, in so far as each completely or almost isolated sphere (or ellipsoid) of protoplasm determines the formation of a separate blastula, and as the number of blastulæ determines the number of embryos. In this way I obtained last year double embryos from a normal egg when I introduced it, in the two-cell stage, into somewhat concentrated sea-water for some time, in which it was unable to segment further. When I brought such eggs back into normal sea-water, each of the two hemispheres broke up into several cells at once. The cells of each of the hemispheres probably adhered to each other, but not to the cells of the other hemisphere, so that an isolation of the two hemispheres was obtained in this way. In one egg I obtained double blastulæ. The method deserves to be worked out more care- 
fully. Herbst has apparently observed the same thing in eggs which he raised in salt solutions which had a qualitatively abnormal constitution. The experiments of Driesch, who found that when the separate cleavage cells are isolated in the four-cell stage, each of the cleavage cells is still able to develop into a completely normal embryo, can also be explained in this way. When the mass of the protoplasmic sphere becomes too small, the formation of a blastula, of course, becomes impossible on geometrical grounds alone, for the size of the cells probably does not fall below a certain minimal rolume during cleavage.

Even though these experiments can leave no room for doubt that an embryo may arise equally well from every part of the protoplasm and from every part of the nucleus, as soon as these parts are isolated to a certain degree, and can assume a spherical or ellipsoid form, the fact nevertheless remains that in many eggs conditions are apparently present which determine the position of the median plane of the embryo and the further orientation of the various parts of the egg. These circumstances may, however, be purely secondary in character, and may depend upon the mass and distribution of the nutritive yolk, the position of the micropyle, and similar secondary conditions. On the other hand, my experiments do not seem to agree with the assumption that each part of an egg can give rise only to a certain part of the embryo.

5 . We must now raise the question whether the formation of twins or double embryos in mammals can come about in a way similar to that described in these experiments. Driesch isolated individual cleavage spheres by rupturing the membrane through shaking. It is probably impossible that an egg can be ruptured in this way in the Fallopian tubes of a mammal. On the other hand, it may be possible that the scheme of my experiments corresponds to natural 
processes in the formation of twins. For I have found that from the moment of the entrance of the spermatozoon into the egg the osmotic pressure of the egg increases greatly. If unfertilized eggs are introduced into dilute sea-water, their volume increases relatively little. As soon as the spermatozoon enters the egg, however, or when an egg which has just been fertilized is brought into the same salt solution, its volume increases very markedly, as I have determined by actual measurements. This fact shows that the spermatozoon brings about chemical changes in the egg which cause an increase in its osmotic pressure. ${ }^{1}$ I will return to the discussion of this point in my more complete description of these experiments.

So far as osmotic pressure is concerned, great differences exist between the eggs from one and the same individual. Even when the sea-water was only slightly diluted, a small percentage of the sea-urchin eggs burst; and I do not doubt that this may occasionally happen in normal sea-water. Whatever may be the actual process in the formation of twins from one egg in mammals, it seems probable that all multiple formations from one egg are caused primarily through complete or partial mechanical, or at least physical, division and isolation of the substances of the egg.

1 Or rather, its power of absorbing liquid. [1903] 


\section{XI}

\section{ON THE RELATIVE SENSITIVENESS OF-FISH EMBRYOS IN VARIOUS STAGES OF DEVELOPMENT TO LACK OF OXYGEN AND LOSS OF WATER ${ }^{1}$}

IT is probable that the series of successive changes in form which we call the development of an animal embryo is accompanied by a corresponding series of physiological changes. While we are well acquairted with the changes in form, so far as their mere morphology is concerned, we know but little concerning the changes in the physiological reactions of the embryo in its various stages of development. It is a well-known fact that the embryo has a greater vitality than the completely developed animals. ${ }^{2}$ Systematic investigations, however, are lacking as to whether this vitality decreases steadily with the progress of the development of the embryo, and as to whether this decrease is the same toward different variables. In order to obtain an answer to these questions, I studied the relative sensitiveness of the fish embryo (Fundulus) to lack of oxygen and loss of water in different stages of its development. I found in general that the embryo is the more sensitive to lack of oxygen, the older it is. Yet the sensitiveness increases more rapidly at first than later. On the other hand, the experiments on the effect of withdrawal of water gave a totally different result. The germ of the embryo is much more sensitive to loss of water in the first stages of its development (during cleavage and before the beginning of the formation of the embryo proper) than after the formation of the blastoderm, and its sensitiveness decreases with the increase in the devel-

1 Pflügers Archiv, Vol. LV (1894), p. 530.

2 Zuntz, Pflugers Archiv, Vol.XIV; and PFLUtger, ibid. 
opment of the embryo. The details are given in the following pages.

\section{THE RELATIVE SENSITIVENESS OF THE EMBRYO TO LACK OF OXYGEN}

1. The fact that embryonal development soon stops, and that the embryo dies without oxygen, has been shown to be true so often by experiment that it is not necessary to discuss it here. ${ }^{1}$ I tried to determine, first of all, how long and how far development could go in various stages of development at the same degree of lack of oxygen; and, secondly, how long the embryo could remain exposed to the same degree of lack of oxygen in different stages of development without losing its power of development. The stages of development which were studied were the following: (1) the freshly fertilized egg; (2) the egg after the formation of the blastoderm, but before the formation of the embryo, about twentyfour hours after fertilization; (3) the egg after the beginning of the formation of the embryo, about forty-eight hours after fertilization (the embryo usually had at this time optic vesicles in which the lens was just being formed); (4) the embryo just after the circulation was established, seventytwo hours after fertilization; and finally various later stages. The egg of Fundulus is especially well adapted to these experiments, because it is very tough, develops fully in the aquarium, and the fish hatches in the aquarium. The entire period of development takes in summer, at a temperature of about $24^{\circ} \mathrm{C}$, about twelve to fourteen days.

The method used in these experiments was similar to that of Bunge in his well-known experiments on the need of oxygen in lower animals." About 6 c.c. of potassium hydroxide and pyrogallol are put into a test-tube (according to Hempel's directions). Into this test-tube is introduced a

1 For the literature on this subject see DüsIng, Pflügers Archiv, Vol. XXXIII.

2 Bunge, Zeitschrift fïr physiologische Chemie, Vol. XIV, p. 322. 
second smaller one containing the eggs and a little sea-water (about 2 to 3 c.c.). The outer test-tube is then sealed. By putting splinters of glass into the bottom of the outer testtube the smaller test-tube is kept above the surface of the pyrogallol. This arrangement allows one to observe the eggs, and at the same time to shake the apparatus in order to accelerate the absorption of oxygen. In some of the experiments the sea-water in which the eggs were contained was boiled, in others this was not done. The result was, however, not very different in the two cases. Lack of proper laboratory facilities did not allow me, however, to study how rapidly and how completely the oxygen is absorbed by the pyrogallol in these experiments. But even if the absorption of oxygen was not complete in these experiments, it was nevertheless equally incomplete in all the experiments. Since we are interested in our experiments only in the relative sensitiveness to lack of oxygen, a quantitative determination of the oxygen absorbed was not absolutely necessary, if the lack of oxygen was only always the same. For the sake of brevity, I will designate the apparatus used for the absorption of oxygen as an "oxygen vacuum." The temperature was usually $22-24^{\circ} \mathrm{C}$. Fundulus eggs require a relatively high temperature for their development.

2. Some eggs of Fundulus were introduced, one-half hour after (artificial) fertilization, into a large number of test-tubes from which the oxygen was absorbed by the method given above. One of these sealed test-tubes was opened at different intervals, and the eggs were compared with eggs from the same culture which had remained in normal sea-water as a control. It was found that cleavage occurred in the oxygen vacuum, and at first even a little more rapidly than in normal sea-water. The latter was, however, probably only the result of the rise in temperature brought about in melting the glass for the purpose of sealing the 
tubes. After twenty-four hours a blastoderm was formed in all the eggs contained in the oxygen vacuum. Then, however, development stopped entirely, while it continued, of course, in normal sea-water. Never was the beginning of an embryo formed in such a series of experiments in the oxygen vacuum. Development continued in the oxygen vacuum only to a point which would have been reached in normal seawater in about fifteen hours.

An egg that ceased to develop in an oxygen vacuum had not necessarily lost its power of development. When brought back into normal sea-water, it could continue its development; only it was necessary that the egg had not been left too long in the oxygen vacuum. Eggs which were introduced into the oxygen vacuum immediately after fertilization could continue their development after they had lain for four days in such a vacuum at a temperature of $22^{\circ} \mathrm{C}$. If they remained in the oxygen vacuum longer than this, they lost their power of development for all time.

In these experiments the eggs were contained in only $2-3$ c.c. of sea-water. One might think that this circumstance had affected the result. I therefore made control experiments in which the eggs were kept in just as little seawater, but in the presence of an abundance of oxygen. In these experiments the eggs developed in an entirely normal way.

3. In the second series of experiments the eggs remained in normal sea-water for the first twenty-four hours after fertilization, and were then introduced into the oxygen vacuum. At this time a blastoderm, but no embryo, was formed. On the next morning an embryo with optic vesicles had formed in nearly all these eggs. The development of those eggs which remained in the oxygen vacuum then came to a standstill, however. Development, therefore, again continued in the oxygen vacuum about as far as a fifteen-hour develop- 
ment would have gone in normal sea-water. These eggs lost their power of development in the oxygen vacuum usually after about forty-eight hours. The maximum that I observed in one case was a continuation of development after a residence of fifty-five hours in the oxygen vacuum. An egg which is introduced into the oxygen vacuum twentyfour hours after fertilization loses its power of development much more quickly than an egg which is exposed to the same degree of lack of oxygen immediately after fertilization.

If embryos are introduced into the oxygen vacuum fortyeight hours after fertilization, the retardation of development becomes more apparent. The formation of the embryo has already begun in these eggs, and the next elements in the further development of the egg would be the formation of pigment and the beginning of the circulation. Pigment is indeed formed, though less than normally, but no circulation is established. After remaining for thirty-two hours in the oxygen vacuum, these eggs had lost their power of development also.

Seventy-two hours after fertilization the circulation is fully developed in an embryo, when it is left in normally aerated sea-water; when at that time the embryo is placed into the oxygen vacuum, in about seven hours the heartbeat becomes very weak. When such an egg was returned to normal sea-water, however, the heart of the embryo began to beat again vigorously almost immediately, at first slowly, but then with so rapid an increase in the rate that the number of beats became relatively great in a few minutes. These eggs lost their power of development in lack of oxygen in about twenty-four hours after introduction into the oxygen vacuum. In no case did such an egg recover when it had remained for forty-eight hours in the oxygen vacuum. The older the embryo, therefore, the more sensitive it is to lack 
of oxygen. The young fish which had just hatched from the egg were still less resistant than the embryos.

These experiments show that the sensitiveness of the embryo to lack of oxygen increases with development. This increase is very great at the beginning of development, so that a four-day old embryo, for example, has just as good, or even a better chance, for continuing its development when it has remained for all this time in an oxygen vacuum than when it has spent only the last forty-eight hours in the vacuum. This apparently paradoxical result is readily explained when we assume that the cells which are formed from the egg-cell during the first stages of cleavage are different chemically from the cells of the embryo which are formed later, so that the latter go to pieces more easily in lack of oxygen than the former. For this reason an egg which has just been fertilized may still be capable of development when it has spent four days in an oxygen vacuum, because it has developed only to the point of the formation of a blastoderm; while an egg which is introduced into the vacuum after the formation of the embryo dies after fortyeight hours. We saw also that development can go on for about fifteen hours in the oxygen vacuum, especially in the first twenty-four hours after fertilization. Whether the conclusion can be drawn from this that cleavage can go on without oxygen, or that the oxygen was not completely absorbed in our experiments, must be determined by further experiments.

\section{THE RELATIVE SENSITIVENESS OF FUNDULUS EMBRYOS TO LOSS OF WATER ${ }^{1}$}

The development of the form of an embryo is a function of processes of cell-division and growth. Both classes of processes are, as in plants, so also in animals, probably a function of osmotic processes. ${ }^{2}$ An accurate knowledge of

1 These experiments were made in the summer of 1892, at Woods Hole.

2 See Part I, p. 191. 
the dependence of processes of development upon the amount of water contained in the cells is therefore desirable.

After some preliminary experiments with sea-water, whose concentration differed comparatively little from that of normal sea-water, had shown that the Fundulus egg is most remarkably independent of the concentration of the seawater, experiments were made with sea-water to which had been added 5, 7.5, 10, and $20 \mathrm{~g}$. of $\mathrm{NaCl}$ to each 100 c.c. of sea-water. Experiments were also made with normal sea-water and fresh water as controls. Freshly fertilized eggs of Fundulus develop perfectly normally in fresh water as well as in sea-water to which $5 \mathrm{~g}$. of $\mathrm{NaCl}$ have been added to each 100 c.c. (that is, $50 \mathrm{~g}$. per liter). When $7.5 \mathrm{~g}$. of $\mathrm{NaCl}$ were added to each 100 c.c. of seawater, a blastoderm was still formed; but only rarely an embryo, and if so the embryo had dwarf dimensions, and its development stopped before the optic vesicles were formed. In solutions to which 10 per cent. $\mathrm{NaCl}$ was added no embryo was formed. The segmentation of the eggs started in this case also, and occurred at first almost as rapidly as in normal sea-water, but it usually ceased at about the thirty-two-cell stage. I expected that these eggs would retain their power of development for some time after this, similarly to those kept in an oxygen vacuum. This was, however, not the case. Freshly fertilized eggs of Fundulus lost their power of development permanently after six to ten hours at a temperature of about $24^{\circ} \mathrm{C}$. in sea-water to which $10 \mathrm{~g}$. of $\mathrm{NaCl}$ had been added to each 100 c.c. When $20 \mathrm{~g}$. of $\mathrm{NaCl}$ are added to each 100 c.c. of sea-water, the power of development of freshly fertilized eggs was annihilated in about three to four hours. The first segmentations, however, took place in such eggs.

When Fundulus eggs are introduced into a 13.5 per cent. $\mathrm{NaCl}$ solution (or, more accurately, sea-water to which $10 \mathrm{~g}$. 
of $\mathrm{NaCl}$ have been added to each 100 c.c.), twenty-four hours after fertilization (before the beginning of the formation of the embryo) not only an embryo is formed in these eggs, but the embryo develops every individual organ; the heart-beats and the circulation are established, and the embryo shows motions after several days. It lives in this concentrated salt solution for ten to fourteen days. Only in three points did the development of such embryos differ from that in normal sea-water: the embryos grew much more slowly in the concentrated sea-water than in normal seawater; secondly, the development of the individual organs was somewhat delayed; and, finally, the yolk shrunk much more rapidly than under normal conditions.

Only a relatively smail percentage of the eggs which were introduced into concentrated sea-water twenty-four hours after fertilization were able to develop. When eggs were introduced, however, into the 13.5 per cent. solution fortyeight hours after fertilization, a larger percentage developed. After the third or fourth day the eggs could be transferred from normal sea-water directly into a 27.5 per cent. $\mathrm{NaCl}$ solution without interrupting development! Development continued for about three or four days. The circulation was not interrupted, even though the beat of the heart had become somewhat slower. The velocity of development and growth was the less the higher the concentration. ${ }^{1}$

We see, therefore, that the sensitiveness to loss of water is incomparably greater during the early period of segmentation of the Fundulus embryo than later, and that even then the sensitiveness decreases somewhat with progressive development. The following experiment is suited to show the great difference in the sensitiveness before and after the for-

1 These experiments may perhaps find their explanation on the assumption that after twenty-four hours the permeability of the egg or the germ-cells is diminished, and hence the $\mathrm{NaCl}$ cannot longer enter fast enough in sufficient concentration to do harm. [1903] 
mation of the blastoderm. Some eggs were introduced into the 13.5 per cent. solution one hour after fertilization; after five hours' cleavage had gone on in these eggs to about the thirty-two-cell stage. Half of these eggs were then returned to normal sea-water, while the remainder were left in the concentrated solutions. Three hours later the latter had lost their power of development, with the exception of three among hundreds of specimens. The other portion of the eggs, after having remained for eighteen hours in normal seawater, were returned again to the 13.5 per cent. $\mathrm{NaCl}$ solution. A large percentage of these eggs formed embryos which remained alive in this concentrated solution for more than a week.

A remarkable phenomenon was observed in the embryos which developed in the very concentrated solutions; namely, that the maximum concentration of the sea-water in which the embryo is able to develop completely is much higher than the maximum of the solution in which the fully developed embryo is able to hatch from the egg. When normal Fundulus embryos are introduced in the second week of their development, into sea-water to which 10 per cent. $\mathrm{NaCl}$ was added they continue their development, but they do not hatch, but die within the egg. This phenomenon shows itself still more definitely when more dilute solutions are used. If $5 \mathrm{~g}$. of $\mathrm{NaCl}$ are added to 100 c.c. of sea-water, the embryos develop in a normal way in this solution, and remain alive for more than five weeks, without hatching. If, however, the eggs are introduced into normal sea-water as soon as the embryo is fully developed (after about two or three weeks), the embryo hatches in one or two days. This fact may be connected in some way with the fact that the fish which has just hatched is more sensitive to the concentrated sea-water than the twoday-old embryo. The former dies in a 13.5 per cent. $\mathrm{NaCl}$ solution in less than twenty-four hours. It can live, how- 
ever, in a 6.5 per cent. solution. In fresh water the embryos hatch just as rapidly as in normal sea-water. The fish is able to live in fresh water.

I know of no other animal which is able to stand such great and sudden variations in the concentration of the seawater as the Fundulus embryo. The question might arise whether this depends upon a peculiar characteristic of the egg membrane or the protoplasm. It can be shown that the protoplasm is relatively insensitive to variations in concentration, while diffusion through the egg membrane occurs very promptly. In order to prove the latter I have to call attention to my earlier experiments on the action of $\mathrm{KCl}$ upon the Fundulus embryo. ${ }^{1} \quad$ The addition of $3 \mathrm{~g}$. of $\mathrm{KCl}$ to 100 c.c. of sea-water brought the heart of one of the older Fundulus embryos to a standstill in a minute. A considerable amount of this salt must, therefore, diffuse through the egg membrane in a very short time. That the protoplasm is very insensitive to variations in concentration can be shown on the spermatozoa. I convinced myself, first of all, of the fact that the unfertilized Fundulus egg can form neither an embryo nor segment. I then introduced eggs, under bacteriological precautions, into sea-water to which $5 \mathrm{~g}$. of $\mathrm{NaCl}$ had been added to 100 c.c. When I added spermatozoa to so concentrated a solution, the eggs developed as in normal sea-water. The movements and the power of fertilization of the spermatozoa must, therefore, be retained in such a solution. The spermatozoa also retain their power of fertilization in fresh water. I believe also that they still penetrated the egg in a 13.5 per cent. solution. I neglected, however, to follow this experiment more accurately, and so must leave this fact undecided for the time being. The fact that the spermatozoon retains its functions in an 8.5 per cent. $\mathrm{NaCl}$ solution is sufficient, however, to show that the independence

1 Part I, pp. 297, 298. 
of the Fundulus embryo to rapid and great variations in the concentration of the sea-water must rest (at least partly [1903]) upon properties of the germ-plasm.

I will not try to say, however, what condition determines that the sensitiveness to loss of water is much greater during the process of cleavage than during the formation of the embryo.

\section{CONCLUDING REMARKS}

The experiments which have just been detailed were made with a view to obtaining further data for a theory of embryonal organization, or - as this is ordinarily termed-for a theory of heredity. It seems to me that some of these theories - for example, Weissmann's theory of determinants assume more for the germ-plasm than it contains. According to this theory, things are already definitely determined in the germ-plasm which, to my mind, are functions of circumstances that first make their appearance in much later stages of development. To give an example: According to the theory of determinants, one would have to imagine that the marking of the yolk-sac of Fundulus is already prearranged by the spatial grouping of the determinants in the germplasm which are responsible for the marking, while I found that the marking is produced by the protoplasm of the chromatophores being compelled through its "chemotropism" to spread over the surface of the blood-vessels. The formation of blood-vessels, as well as the formation of pigment-cells, may perhaps be traced back to the original germ, but the spatial arrangement of the pigment-cell is, as we have seen, the effect of a stimulus which the fully developed vessels, or rather the blood which they contain, exercises upon the fully developed chromatophores. These facts led me to the idea that the chemical circumstances determining organization do not all exist ready-formed in the germ-plasm, but arise gradually in the different stages of development. The 
development of an embryo would, according to this, be in a physiologico-chemical sense an epigenesis and no evolution. In order to test this idea, I made experiments on the relative sensitiveness of the embryo in the various stages of its development. I thought that sudden changes in the sensitiveness during the transition from one developmental stage to another would speak for epigenesis. Such a change in reaction was, indeed, found in the experiments on loss of water.

I have begun experiments similar to those on Fundulus on Perca fluviatilis. The physiological reactions of the embryo of Perca are, however, very different from those of Fundulus, as was to be expected from the beginning 


\section{XII}

\section{ON THE LIMITS OF DIVISIBILITY OF LIVING MATTER ${ }^{1}$}

1. The progress which has been made in physics and chemistry as the result of our modern conceptions of molecules and atoms suggests the possibility that a more definite insight into the limits of divisibility of living matter might also be of importance for the development of physiology. As a criterion for "living matter" we might use the irritability or spontaneity. But as the "spontaneity" of living matter is in its simplest form (in Amœbæ) apparently not different from the physical phenomenon of spreading, for this criterion the limits of divisibility of living matter coincide with the limits of this purely physical phenomenon. But spontaneity is neither the deepest nor the most essential life-phenomenon; development_or, in other words, growth, organization, and reproduction - occupies this place. If we ask how the ultimate elements of living matter are constituted which still possess the specific morphogenetic properties, the excellent papers of Nussbaum give us a qualitative answer. This investigator found in experiments on the divisibility of an Infusorian, Gastrostyla, that only such pieces are able to regenerate into a complete animal as contain nuclear material.

For the preservation of an Infusorian it is immaterial whether it is divided longitudinally, transversely, or obliquely; if only a portion of the nucleus is retained, the fragment regenerates into its original form in less than twenty-four hours, depending upon the temperature. As soon as twenty minutes after division the cut edges form new cilia, and upon the following day each of the pieces containing nuclear material possesses from four to six nuclei and nucleoli, and all the ciliary appendages characteristic of the species. ${ }^{2}$

1 Pflügers Archiv, Vol. LIX (1894), p. 379.

2 NUssBaUM, Archiv für mikroskopische Anatomie, Vol. XXVI, p. 514. 
A piece without the nucleus "cannot develop into the form characteristic of the species, and growth does not take place." Yet a piece without the nucleus, as Nussbaum found, can move for a long time; nuclear substance is, therefore, not necessary for "activity" or "spontaneity." Nussbaum draws the following conclusions from his experiments:

(1) Nucleus and protoplasm can live only when united; isolated they die after a longer or shorter time. (2) For the maintenance of the morphogenetic energy of a cell the nucleus is indispensable. (3) Each of the energies produced by a cell depends upon a substratum that can be divided.

If I understand Nussbaum correctly, the latter statement means that a part of the nucleus and of the protoplasm is sufficient to render possible all the life-phenomena of the cell.

Finally, I wish to quote also the following from the work of Nussbaum:

The cell is not the ultimate physiologic unit, even though it must remain such for the morphologist. We are, however, not able to tell how far the divisibility of a cell goes, and how we can determine the limit theoretically. Yet for the present it will be well not to apply to living matter the conceptions of atom and molecule, which are well defined in physics and chemistry. The notion micella introduced by Nägeli might also lead to difficulties, as the properties of living matter are based upon both nucleus and protoplasm. . . . The cell consequently represents a multiple of individuals. (P. 522.)

The conception which we must therefore form of the nature of the simplest elements of living matter capable of development is this, that it consists of a system of at least two different substances, of which the one is contained only in the nucleus and the other only in the protoplasm. The experiments of Nussbaum have been repeated and amplified by a large number of careful observers. 
Nussbaum's observations and conclusions were, so far as $\mathbb{I}$ know; corroborated in every particular.

2. In all these experiments the answer as to the quantitative limits of the divisibility of living matter has not been obtained. It is of great importance, however, to have a clear idea of how large the smallest piece of nucleus and protoplasm is that is capable of development. Is it of the order of magnitude of two or more micellæ, or is it of the order of magnitude of a considerable fragment of the cell? I have tried to obtain an answer to this question in the seaurchin egg. Pflüger has stated already that the egg which had been considered as a unit can give rise to many individuals. ${ }^{1}$ The experiments of Driesch, which we shall mention immediately, and my own experiments on the production of double and multiple embryos from a single egg, correspond with the views of Pflüger. The question, therefore, naturally arose as to how many embryos can arise from an egg, and to determine in this way the limits of the divisibility for one kind of living matter; namely, the egg. The simplest way of determining what fraction of the substance of the sea-urchin egg is still able to develop into a normal embryo seems at first sight to be that in which one of the cells of the egg in various stages of cleavage is isolated, and the last stage in which a single cell is still able to develop into a pluteus is determined. (The eggs cannot usually be kept beyond the pluteus stage in an aquarium.) The cleavage cells become smaller as cleavage progresses and the number of cells increases into which the egg divides. In another connection Driesch has shown that one of the cells from the four-cell stage of the sea-urchin egg is still able to develop into a pluteus. ${ }^{2}$ For our purposes, however, the methods and results of Driesch cannot be un-

1 Pflügers Archiv, Vol. XXXII, p. 562.

2 DrIEsch, Zeitschrift für wissenschaftliche Zonlogie, Vol. LV, pp. $5 \mathrm{ff}$. 
reservedly employed, as it is questionable whether one of the cells of the eight- or sixteen-cell stage can really be considered as identical with the eighth or sixteenth part of the unsegmented egg. It is possible that through the process of segmentation the substance of the egg is separated into unhomogeneous parts. It is further possible that the metabolism during segmentation changes the material contained in the various cleavage cells unequally. This might result, for example, in this, that one of the cells of the eight-cell stage would no longer be able to develop into a complete embryo, while one-eighth of the same egg before cleavage might have been able to form a complete embryo. A short time ago I published a method which enables us to divide the unsegmented fertilized egg into small pieces capable of development. ${ }^{1}$ The method consists in bringing the sea-urchin eggs, after fertilization, into seawater which has been diluted through the addition of 100 per cent. of its volume of distilled water. The contents of the egg absorb water rapidly, and the thin egg-membrane ruptures at one or more points; a portion of the protoplasm flows out of these ruptures, which assumes a spherical form, and usually remains connected with the egg. (Fig. 74.) When the eggs are returned to normal seawater, they begin to segment, the extraovate as well as the protoplasm which has remained in the egg form separate blastulæ, and twins result from the egg. These may either remain attached to each other or separate later on. The latter usually occurs. It is possible, however, that not only two but several protoplasmic drops may exude from the egg, and in this way more than two embryos may develop from one egg. Finally, in some cases where only one extraovate exists, a separation of groups of cells may occur during segmentation which leads to the production of

1 Pfï̈gers Archiv, Vol. LV; and Biological Lectures Deiivered at Woods Hole. 1893 (Boston: Ginn \& Co.). 
more than two embryos from an egg. If the eggrs are made to burst before segmentation begins, only one nucleus is prosent, and in consequence either the contents of the egg or the extraovate must be without a nucleus. I have already mentioned in my earlier papers that in the course of the segmentation nuclear material gets into that portion of the protoplasm which was originally free from it. Occasionally the extraovate is cut off before nuclear division occurs. Nevertheless, cleavage occurs. From the observations of $O$. and R. Hertwig and of Boveri we may assume that in these cases a spermatozoon has entered the protoplasm. The nuclear material which is introduced in this way suffices to inaugurate the process of cleavage.

3. In these experiments it is natural, of course, that the extraovate, as a rule, does not contain exactly one-half the mass of the egg. Those cases in which the extraovate and the contents of the egg differ greatly in size are well adapted to decide how large an amount of the egg-substance is just sufficient to give rise to a normal pluteus. I followed the development of selected individual eggs with their extraovates in a drop of water contained in a moist chamber. I also examined very carefully from day to day cultures of such eggs kept in large vessels, and determined the size of the smallest plutei. Finally, I studied also from day to day the fate of these small fragments. The results of these observations, which I pursued uninterruptedly for two months last year and again for two months this year, were very definite, and may be expressed as follows:

a) The smallest normal plutei which arose from fragments of an egg were linearly about half the size of the plutei arising from a whole egg of the same culture. Their volumetheir density being considered the same - was therefore about one-eighth of that of a normal pluteus.

b) Smaller fragments of an egg than these developed into 
blastulæ and reached the gastrula stage later than the larvæ formed from entire eggs. In the most favorable case irregular precipitates of calcium salts were formed, but the changes

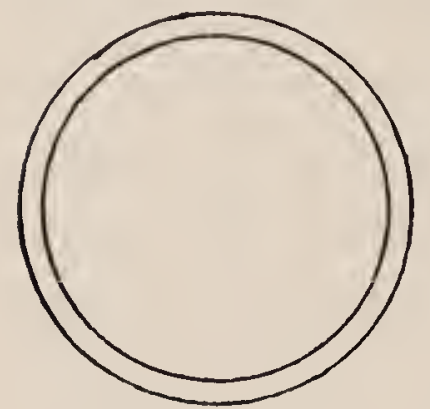

FIG. 73

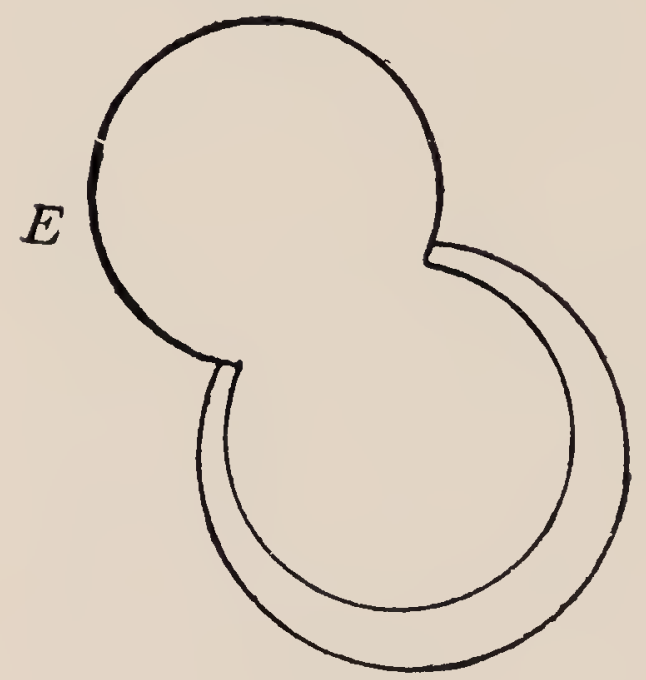

FIG. 74 of form characteristic of the pluteus stage did not occur. These monsters did not develop beyond the spherical form of the

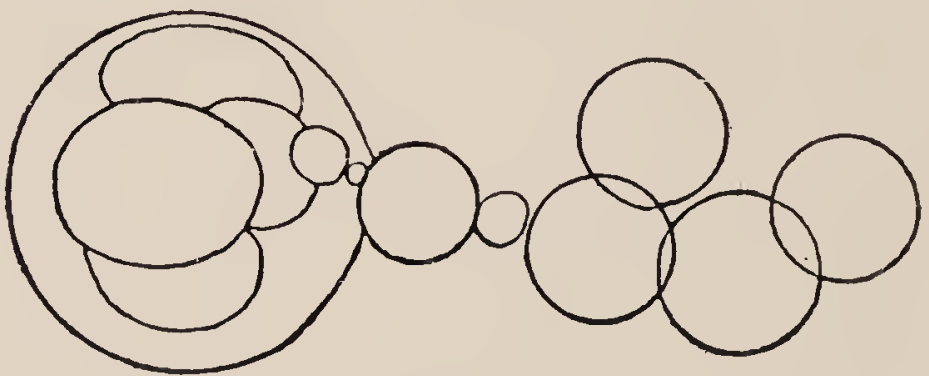

FIG. 75

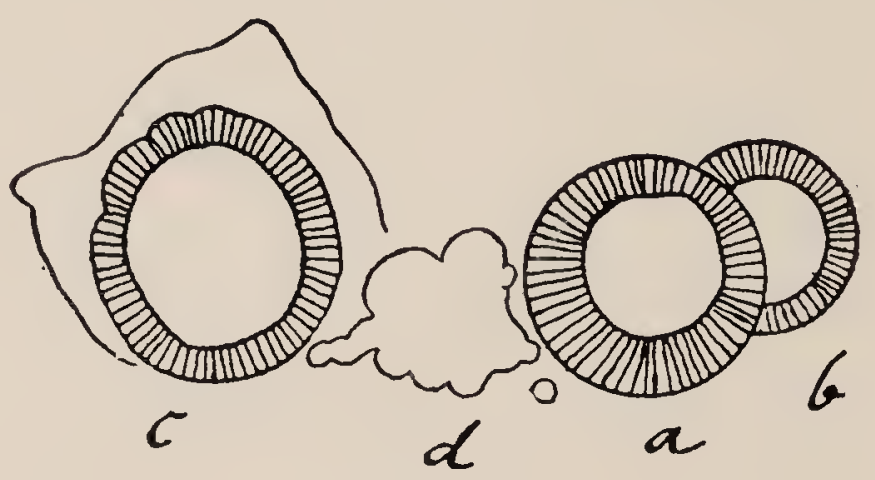

FIG. 76

gastrula. They lived, however, as long as the normal plutei, and, so far as motility was concerned, were comparable to normal embryos.

4. I will now enter upon these observations in somewhat greater detail, and will use for this purpose a series of drawings, all of which were made with the camera lucida at about the same magnification, Fig. 73 gives the form of a normal fertilized egg with its membrane. Fig. 74 gives the form of an egg with the extraovate $E$ which has burst in sea-water. I have described, in a former paper, the first processes of cleavage which occur in such an egg. We will now follow the development of multiple embryos from such an egg. Fig. 75 shows a bursted egg in the twelve-cell stage. It can be seen that the micromeres form a bridge between the extrao- 
vate and the egg. Four hours later this egg was in the condition shown in Fig. 76. The cleavage cells lying within the egg in Fig. 73 have developed into the blastula $c$. The micromeres and the large cells inclosed between them in Fig. 75 have developed into a misshapen mass of cells $d$ in Fig. 76 ; each two of the four large cells of the extraovate have led to the development of the separate blastulæ $a$ and $b$, so that we obtained from this one egg three blastulæ and a mass of

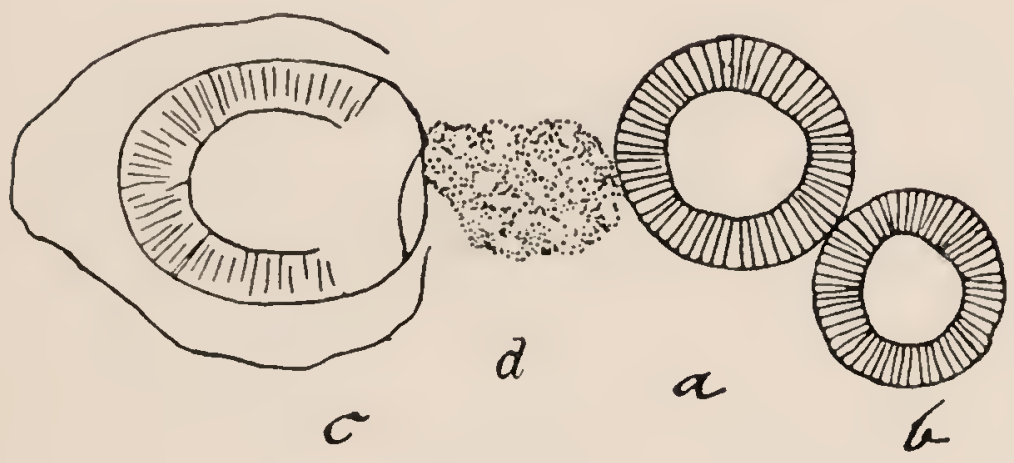

FIG. 77

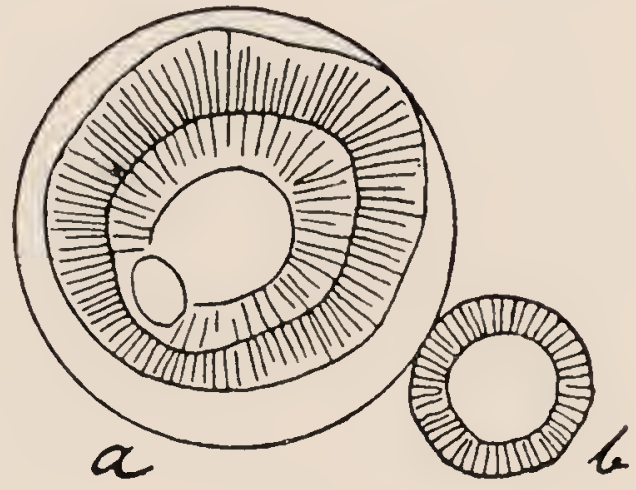

FIG. 78

shapeless cells. ${ }^{1}$ Fig. 77 shows the same egg twenty-four hours later. The largest of the blastulæe $c$, which has remained within the egg-membrane, has developed into a gastrula, while the two smaller blastulæ $a$ and $b$, which have remained outside the egg, have developed no further. A short time after this drawing was made all four pieces began to swim about in the drop. The formation of the blastula therefore occurred at the same rate in the smaller masses as in the larger one. I may add that it also occurred at the same rate as in the eggs whose membrane had not burst. One notices, of course, that eggs which are placed into dilute sea-water, and consequently go into "water rigor," do not all recover and begin to segment at the same time after they are returned to normal sea-water. Under these conditions an extraovate

1 It often happened that the cells of the extraovate formed not one, but two or more, blastuler. The sliding motions of the cells are not restricted in the extraovate, anci can therefore lead to various groupings of the cell-masses. Inside of the eggmembrane this origin of twins also occurs, but more rarely. The membrane restricts the sliding motions of the cells. I shall discuss this question in greater detail later in a paper on the formation of double embryos. 
may begin to segment somewhat later than the rest of the egg. The influence of the quantity of the egg-substance upon the formation of the gastrula is, however, an unquestionable one. The substance which remained within the egg has developed in Fig. 77, twenty-four hours after fertilization, to the gastrula stage. According to Fig. 75, its mass is about twice as great as that of each of the two blastulæ which arose from the extraovate, and which at this time had

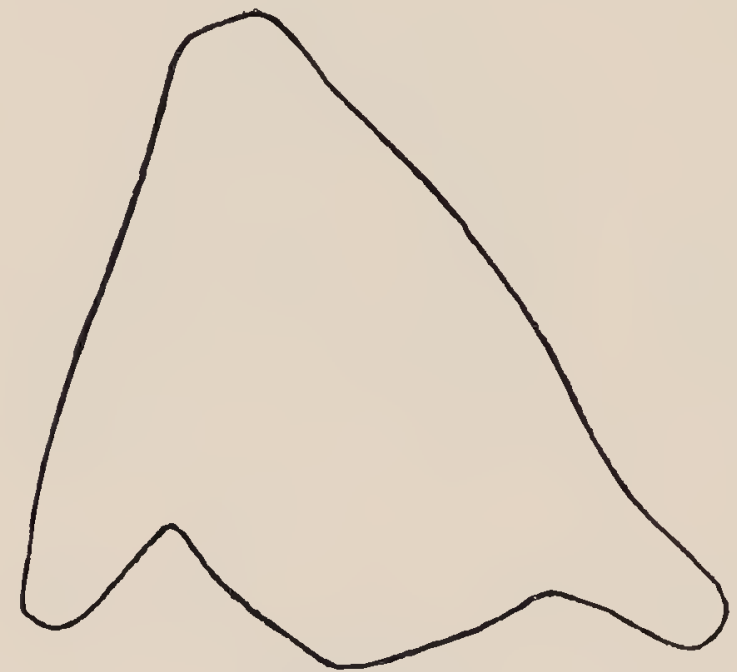

FIG。 79

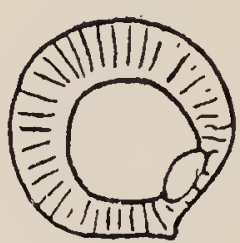

FIG. 81

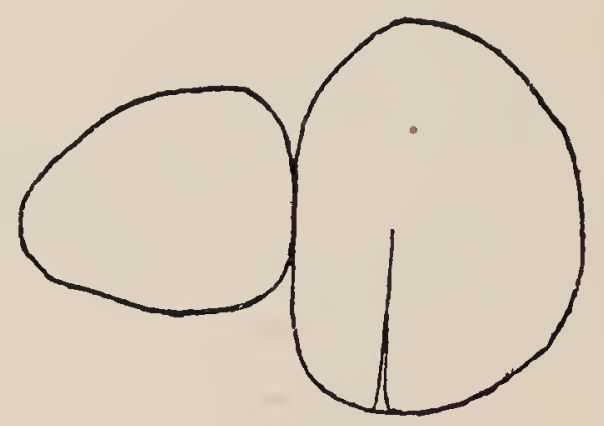

FIG. 80

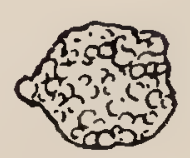

FIG. 82

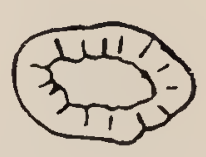

FIG. 83

not yet reached the gastrula stage. I have observed this over and over again; as, for example, in Fig. 78, where the small extraovate $b$ has formed a blastula, while the rest of the egg $a$ has formed a gastrula. The cultures kept in a drop of water always died in the course of the second or third day. The large pieces reached the pluteus stage during this time, while the smaller pieces remained in the blastula or gastrula stage. In order to follow the further fate of these small fragments of the egg after the second or third day, I had to rely on the material from the cultures kept in the larger dishes. Figs. 79-83 represent the relation between mass and development in a culture two days old. Fig. 73 shows the size of a fertilized but undeveloped egg of this culture two days old; Fig. 79, the size of a pluteus that had developed from an entire egg, which, however, had been 
subjected to the same treatment with dilute sea-water as the pieces which are about to be described. Fig. 80 is a double embryo which had arisen from a bursted egg. The two embryos are unequal in size, and the larger is ahead of the smaller in development in so far as a deposition of needles of calcium carbonate has begun in the latter. Both, however, are less developed than the pluteus which has arisen from a whole egg. Fig. 81 arose from a fragment which was smaller than half the egg of Fig. 73. It is an early gastrula stage. Figs. 82 and 83 are still smaller fragments of an egg, which have, however, reached only the blastula stage. These examples are not especially selected, but they represent only what the observ-
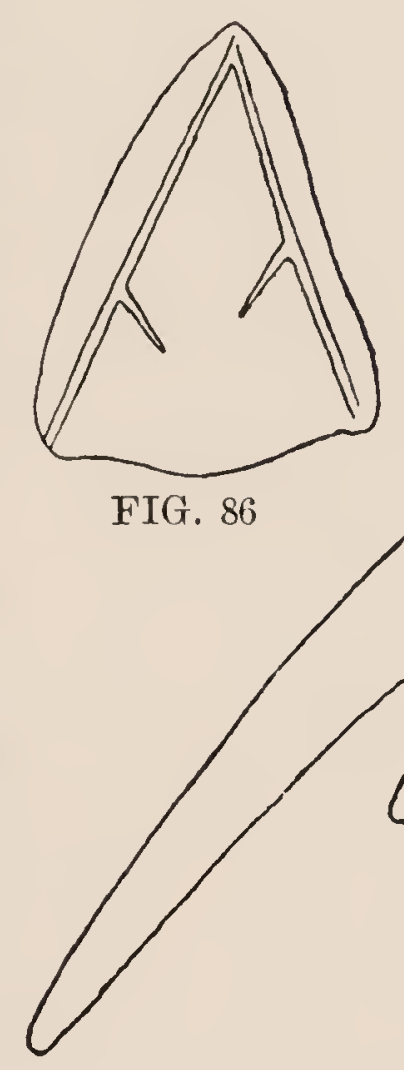

FIG. 87

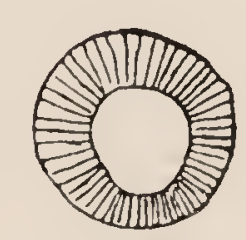

FIG. 84

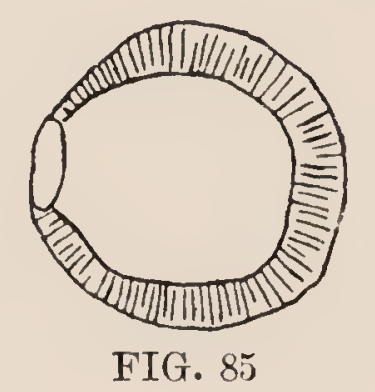

er will find in any sample from such a culture.

Do these small pieces develop to the pluteus stage? Two days later $I$ found the conditions in this culture as shown in Figs. 84-87. Fig. 84 is one of the smallest fragments living at this time. It is only a blastula. Fig. 85 represents a larger piece in the gastrula stage, but without any evidence of a skeleton. Fig. 86 shows the smallest pluteus; Fig. 87, 
a medium-sized pluteus which arose from a whole egg. If the linear dimensions of both plutei are compared, we find that they are about in the relation of one to two, which at equal density would correspond to a relation of their masses of one to eight. The smaller fragments generally do not go into a normal pluteus stage, but form only irregular needles of calcium salts, retaining, however, the spherical shape of the young gastrula. Figs. 88 and 89, for example, are such gastrulæ five days old from the culture under discussion. Fig. 90 is a smallest pluteus of the same age. These gastrulæ with skeletal needles may grow, but their exter.

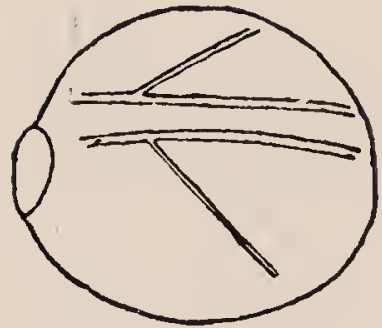

FIG. 88

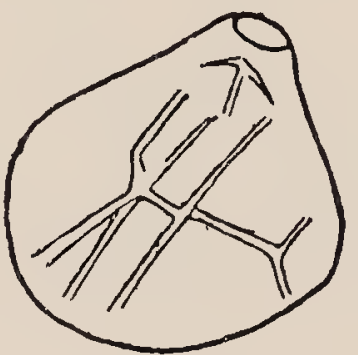

FIG. 89

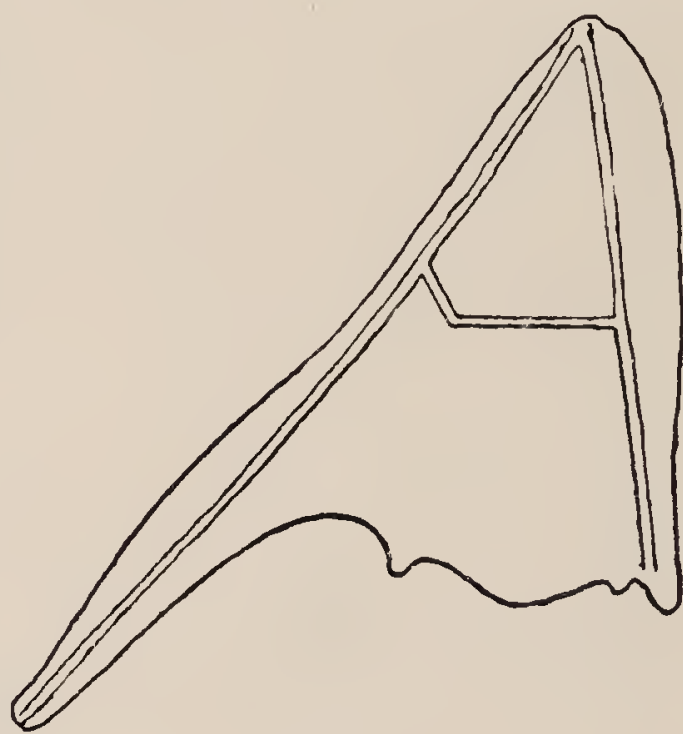

FIG. 90

nal form usually remains unchanged, and the skeleton remains abnormal. Finally, I should like to add a few words regarding the fate of such misshapen heaps of cells as shown in Fig. 76d. Their outer surface forms cilia, like that of normal embryos, and like the latter they move with great rapidity in the aquarium; they seem to live as long as the plutei. These groups of cells represent free-swimming tumors, teratomas, which have arisen because of sliding motions of cells which could occur on a larger scale on account of the lack of a membrane.

5. We have seen, therefore, that the smallest pluteus with normal shape which arose from a fragment of an egg had about one-eighth the volume of the normal average-sized pluteus which sprang from an entire egg. I may add that I have never observed smaller normal plutei than this. In 
determining the limits of divisibility of living matter, it is of importance to decide whether such a pluteus arises from a piece of an egg the mass of which amounts also to oneeighth of that of the total egg. We must therefore know whether the embryos originating from fragments of an egg grow more rapidly or more slowly than those which arise from an entire egg. Now, as I have mentioned before, it is a general rule that the smallest pieces after having attained the blastula stage develop less rapidly than those which are formed from an entire egg. In my earlier experiments on growth and regeneration in Tubularia I found that development and growth are functions of the same variables; there can be no doubt that to a certain extent development is only a function of growth. It is therefore probable that the embryo arising from a small fragment of an egg grows less rapidly than that arising from an entire egg. It is therefore also probable that a pluteus the mass of which amounts to only one-eighth of that of a normal pluteus has developed from a fragment of an egg which contained more than oneeighth of the substance of the entire egg. I will, however, not deny the possibility that a later observer may perhaps find a still smaller pluteus, even though the large number of my experiments renders this scarcely probable. ${ }^{1}$ But I believe that even in such a case the limit which I have given will suffer no great reduction. The divisibility of the egg is therefore very limited, if one demands that the fragment shall develop into a pluteus.

6. So far as my present experiments are concerned, I am not yet able to say where the limits of divisibility lie, when it is only required that the piece develop into a blastula. The tiniest pieces of isolated egg-protoplasm still divided if

1 Boveri has since stated that he found a pluteus whose lincar dimensions were only one-third of those of a normal pluteus of the same culure. But as a slight retardation in the growth of the arms may easily lead to such a result, I think that, on the whole, the limits observed by me in many experiments will be nearer the truth than the one exceptional observation made by Boveri. [1903] 
they contained nuclear substance. So far as I was able to see, very small pieces developed into blastulæ. It is probable, therefore, that the egg can be divided much further when we wish only blastulæ to develop than when we wish the fragments to reach the pluteus stage. It seems probable, however, from my observations, that the blastula must have attained a certain size kefore it is able to develop into a gastrula, so that the limit for the amount of egg material necessary for development into the gastrula stage is probably greater than that necessary for the development of a blastula.

7. We can here, in passing, answer a question which we have touched upon before, but which does not necessarily belong to our subject, namely: Do qualitative changes of a sufficiently profound nature occur in the cells during segmentation (besides the mere increase in their number), which interfere with the divisibility of the egg? As has been said, Driesch was able to convince himself that an isolated cell of the four-cell stage could still develop into a pluteus; but this seems not to have been possible when he isolated one of the cells from the eight-cell stage. He attributed this, very correctly, to the amount of substance present. Others, however, were inclined to conclude from such experiments that in the eight-cell stage the differentiation in the individual cells had progressed so far that they could give rise only to individual tissues, but not to entire embryos. But it is apparent that the limits of divisibility of the egg in Driesch's experiments harmonize with those found in my own. If, therefore, one of the cells from the eight-cell stage is no longer able to develop into an entire embryo, this is to be attributed to the fact, as shown by my experiments, that the amount of material present in one cell in this stage does not suffice to form a pluteus. Besides this, of course, a differentiation might have occurred in the cells. I have made experiments which show that this cannot 
be the case to a sufficient extent to interfere with the development. Fertilized eggs of Arbacia were kept in normal sea-water until they had reached the eight-, sixteen-, and thirty-two-cell stages, when the eggs were put into water which had been diluted to a sufficient extent. The membrane burst, and a portion of the egg contents flowed out, as in the case of the unfertilized egg, only with this difference, that in this case the extraovate consisted of a larger number of cells. Nevertheless, the result was the same as in eggs which were made to burst before cleavage had begun. When the separated pieces were sufficiently large-greater than one-eighth of the entire mass of the egg - they developed into a pluteus; when they were smaller than this, they reached only the gastrula or blastula stage. If a differentiation had occurred in the eight-cell stage, so that the individual cells could give rise only to certain tissues, organs, or regions of the body, we would expect that, when only about eight of the cells from an egg in the thirty-twocell stage were separated from the main mass, we should obtain only a conglomeration of different fragments of tissues, organs, etc., but not an entire embryo, which is, however, not the case. It may also happen, as I have just mentioned in the case of the uncleaved eggs, that the cells in their sliding motions distribute themselves so that they do not form a blastula; but this does not occur oftener in these eggs than in those which were ruptured before cleavage began.

8. After this digression we will return to our main theme, and ask the question whether it makes any difference what portion of the protoplasm is cut out of an egg. It might be that the egg is not completely isotropic. The protoplasm always begins to flow out at the point at which the membrane is ruptured. It can easily be shown, however, that the seat of the rupture may be at any point in the 
cell-membrane, and that it certainly has no relation to the orientation of the first cleavage plane. For when we first allow the eggs to develop in ordinary sea-water into the twocell stage before bringing them into dilute sea-water, it can be noticed that the first cleavage plane may lie in any posi-

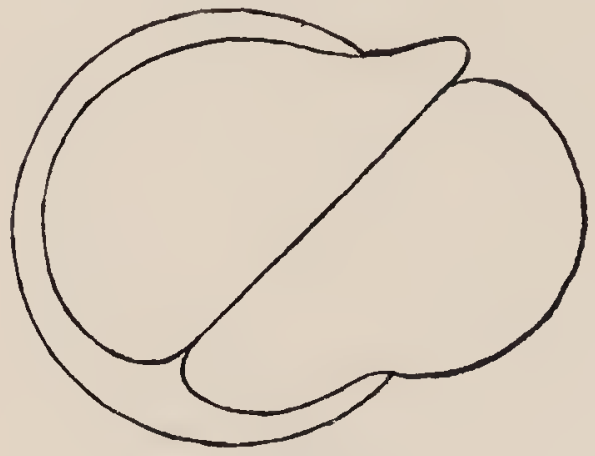

FIG. 91 tion with regard to the point of rupture; the material lying nearest the rupture will be that which flows out. Figs. 91-94 are drawings of eggs the membranes of which were made to rupture in the two-cell stage, and which illustrate what has been said more clearly than words. Since the extraovate develops in all cases, if it is only sufficiently large, we must conclude that, so far as the question of divisibitity is concerned, the protoplasm must be considered an isotropic substance.

9. What conceptions can we form of the nature of the smallest elements of living matter which are capable of development? As Nussbaum has shown, every attempt that has been made of assuming as the ultimate elements of living matter something analogous to the atom and the molecule has failed, for the simple reason that two different substances, nucleus and protoplasm, are necessary. One might assume that a combination of two different "micellæ" - one composed of nuclear material, the other of proto-

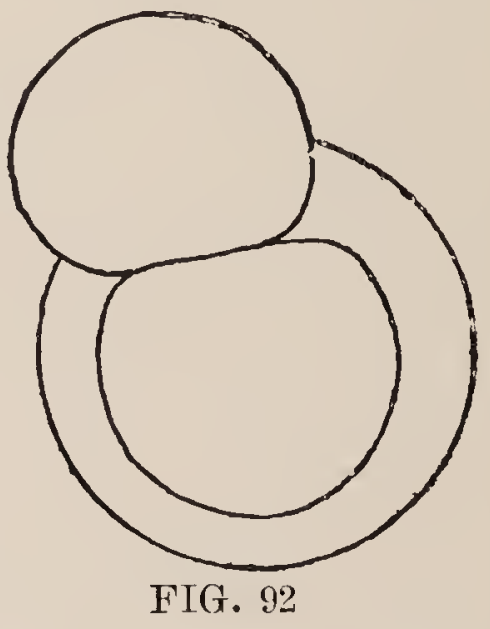
plasm - might represent the smallest living element. Our experiments show that such an idea would be entirely wrong, when full capacity for development is taken as the criterion of living matter, inasmuch as a very considerable quantity of substance is necessary for full development-an amount 
which, according to our experiments, is not far remored from the limits of macroscopic visibility. I have emphasized the fact that for geometrical reasons alone a certain amount of substance must be present before it is possible to form a pluteus. But the lowest limit actually found is reached very much earlier than that re-

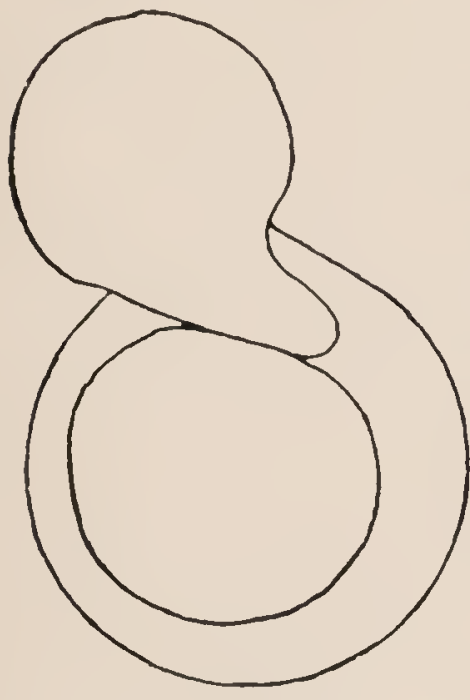

FIG. 93 quired from geometrical considerations alone. Since it has been demonstrated that the ultimate source of all energy for life-phenomena is of a chemical nature, we must conclude from our experiments that the ultimate unit of living matter is such a quantity of substance as is capable of developing that amount of energy which is necessary for that lifephenomenon which is used as a criterion. In this we find a natural explanation of why the amount of substance necessary for the formation of a pluteus must be much larger than the amount of substance which is sufficient for the formation of a blastula, inasmuch as a larger amount of living matter represents also a larger amount of energy. It follows from this, also, that when one is satisfied with spontaneity or irritability as the criterion of living matter, the ultimate unit of living matter is not only much smaller quantitatively, but also different qualitatively, as the protoplasm alone suf-

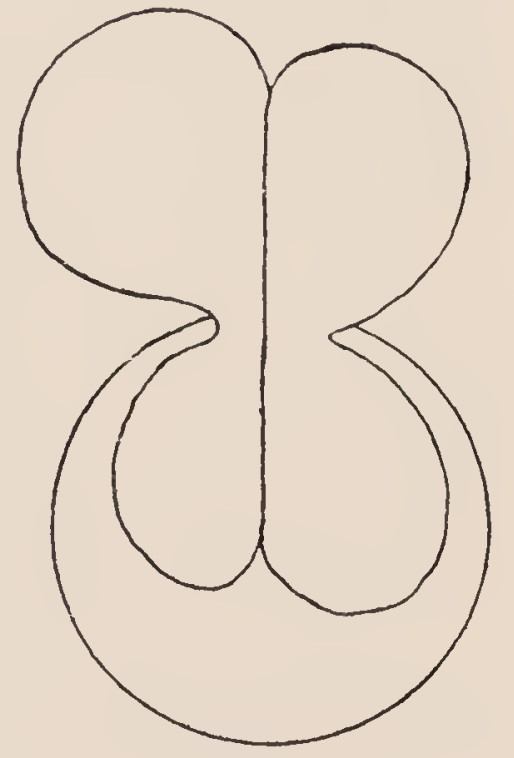

FIG. 94 fices for this. In the case of ultimate units we not only deal with masses which represent a definite amount of chemical energy, but we have every reason for assuming that the mode of liberation of this energy follows a definite order, which is possibly the same for all life-processes. Our fur- 
ther insight into the nature of these ultimate elements will consequently be dependent upon a knowledge of this order.

This significance of the quantity of living substance as the carrier of a definite amount of energy is also apparent in the regeneration of multicellular animals. According to the experiments of Nussbaum, "at least one ectoderm, one entoderm, and one cell from the intermediary germinal layer is necessary" for the regeneration of a Hydra capable of reproduction. ${ }^{1}$ But this minimum gives us only a qualitative limit, in so far as the three qualitatively different elements are necessary. So far as the quantity is concerned, it must be said that a very large multiple of each of these three elements is necessary for regeneration. In experiments on Tubularia which Miss Bickford made in my laboratory two years ago $^{2}$ it was found that pieces $1 \mathrm{~mm}$. long from the stem of this Hydroid are no longer able to regenerate into complete Tubulariæ; either only a simple polyp without stem and root is formed, or a peculiar heteromorphous formation, a sort of Janus head, occurs, consisting of two polyps connected with each other by their aboral ends, while the stem and root are missing between them.

That the smallest amount of matter capable of development must have a different absolute size in different organisms - that, for example, it must be smaller for a coccus than for an Arbacia egg-need not be specially mentioned.

10. The results of our observations are briefly as follows:

a) The limits of divisibility of living matter must vary according to the character of the life-phenomena used as a criterion of life. Each quantity of living matter is the bearer of a definite quantity of energy.

b) The smallest fraction of an unsegmented egg of Arbacia necessary for the formation of a pluteus is about

1 Archiv für mikroskopische Anatomie, Vol. XXXV (1890).

2 E. BICKFORD, Journal of Morphology, 1894. 
one-eighth of the mass of the entire egg (nucleus plus protoplasm).

c) The amount of substance necessary for the formation of a blastula is much smaller than that necessary for the formation of a pluteus; for the formation of a gastrula more substance is probably required than for the formation of a blastula.

d) It does not matter which position the fragments of an egg of Arbacia occupied in the intact egg; so far as divisibility is concerned, the protoplasm of the Arbacia egg can certainly be considered as isotropic.

e) Since the limits of divisibility are almost the same in the unsegmented egg as in the first stages of segmentation (the thirty-two-cell stage included), it follows that $(a)$ no qualitative changes occur in the egg during the early stages of segmentation which restrict the development of fragments of the egg, and that $(\beta)$ the individual cleavage cells, so far as the limits of divisibility of the substance of the egg are concerned, may be considered as equal. (In other respects, however, differences may exist between the individual cleavage cells.) 


\section{XIII}

\section{REMARKS ON REGENERATION ${ }^{1}$}

\section{ON THE REGENERATION OF THE BODY IN PANTOPODS}

1. So FAR as I know, it is generally held that in Arthropods regeneration is possible only in the appendages, while segments of the trunk are not regenerated. According to experiments which I made at Woods Hole last year, Pantopods, or at least one form of this group, Phoxichilidium maxillara, form an exception to this rule.

The trunk of Phoxichilidium maxillara (Fig. 95) is about $1 \mathrm{~cm}$. long. The animal remains alive for weeks in a dish of sea-water. It is, like most of the free-moving inhabitants of the surface of the sea, positively heliotropic. ${ }^{2}$ If the body of one of these animals is cut in two by a transverse incision (at $a$, Fig. 95), each of the pieces is still capable of locomotion. If the pieces are exposed to the light, it is found that the oral piece continues to be heliotropic, while the aboral piece moves about independently of the light. The latter do not show much tendency to progressive motion.

That injured Pantopods can remain alive has already been observed by Dohrn. The latter writes:

I have observed that individuals continued to live for days even when all of the extremities have been cut off. I have even cut a female specimen of Barana castelli in two, dissected the anterior portion of the body, and kept the posterior portion carrying the extremities V to VII, alive for fully four weeks. ${ }^{3}$

1 Archiv für Entwickelungsmechanik der Organımen, Vol. II (1895), p. 250.

2 Part I, p. 1.

3 A. Dohrn, Fauna und Flora des Golfes von Neapel; III, "Pantopoda" (Leipzig, 1881), p. 81 . 
I was macquainted with this observation of Dohru's, and so made more exhaustive experiments on the subject. I succeeded not only in keeping alive for weeks pieces consisting of several segments, as did Dohrn, but even single segments of the body with only one pair of legs. Animals which I had cut longitudinally did not remain alive, yet I consider it possible that further experiments in this direction may be accompanied by better results.

2. In animals in which the body was divided between the second and third pair of legs (at $c$, Fig. 95) the segments of the body which were cut off were regenerated. This regeneration was especially marked in the oral halves of the animals which had to regenerate the posterior segments (Figs. 96, 97). In the aboral halves of the animals I

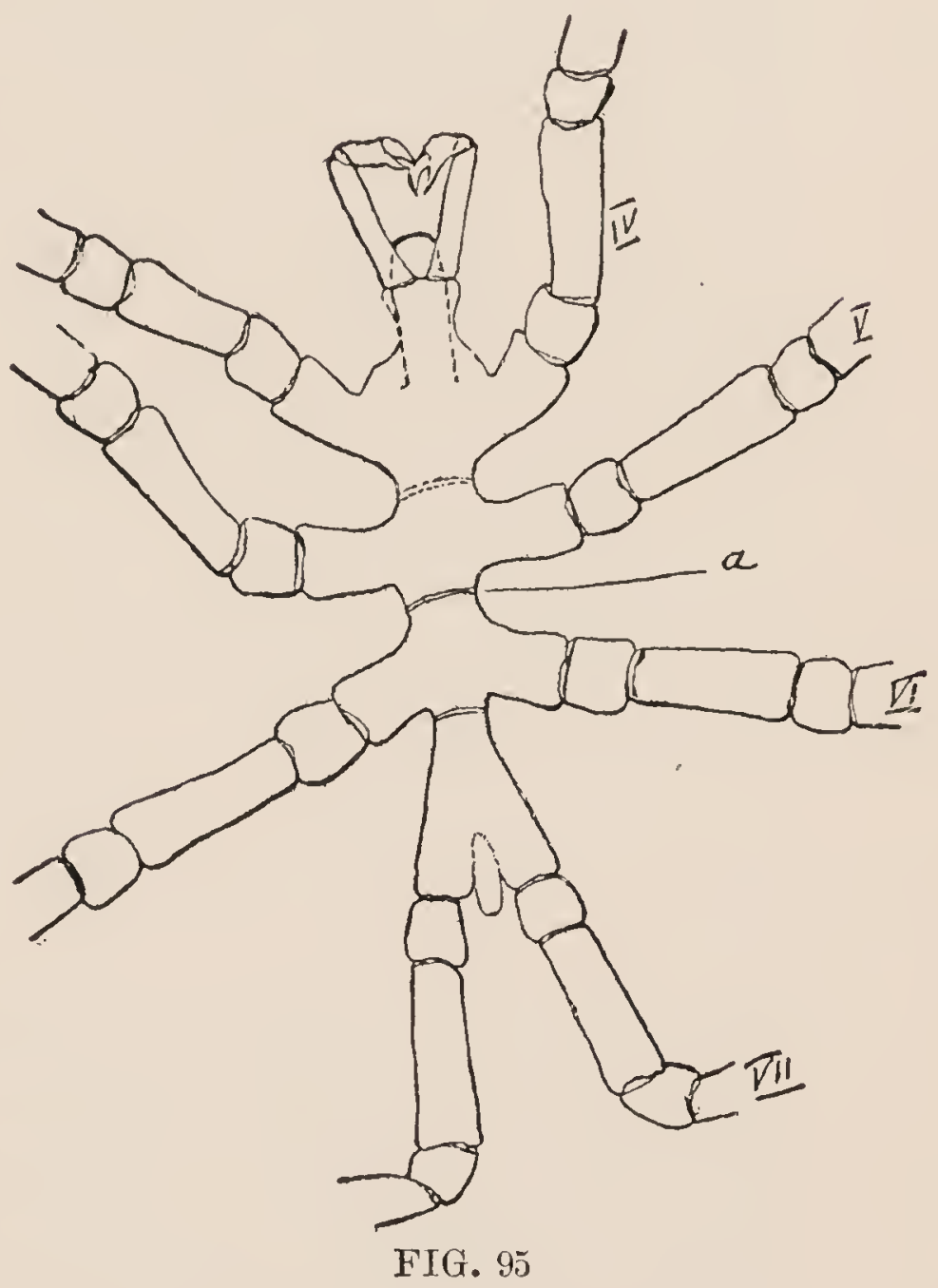
could discover only a swelling of the anterior end. In some cases a complicated regeneration was recognizable in this swelling. I had to discontinue my observations before the process of regeneration was completed.

We will now discuss the regeneration of the posterior segments of the body in somewhat greater detail. The regenerated piece $a b$ in Fig. 96 consists of three segments; yet the constriction at $c$ is not as deep as is usual at one of these joints; in Fig. 97 the regenerated piece $a b$ consists of four segments instead of the three which were expected. The 
presence of a supernumerary segment leads one to suspect that the regenerated piece might perhaps have developed into a leg in the course of time; that, in other words, we might have had to do with a heteromorphosis, namely, the formation of a leg in the place of the body segment which had been cut off. Hoek, ${ }^{1}$ however, states that the abdomen of Ammother not infrequently shows traces of a segmentation.

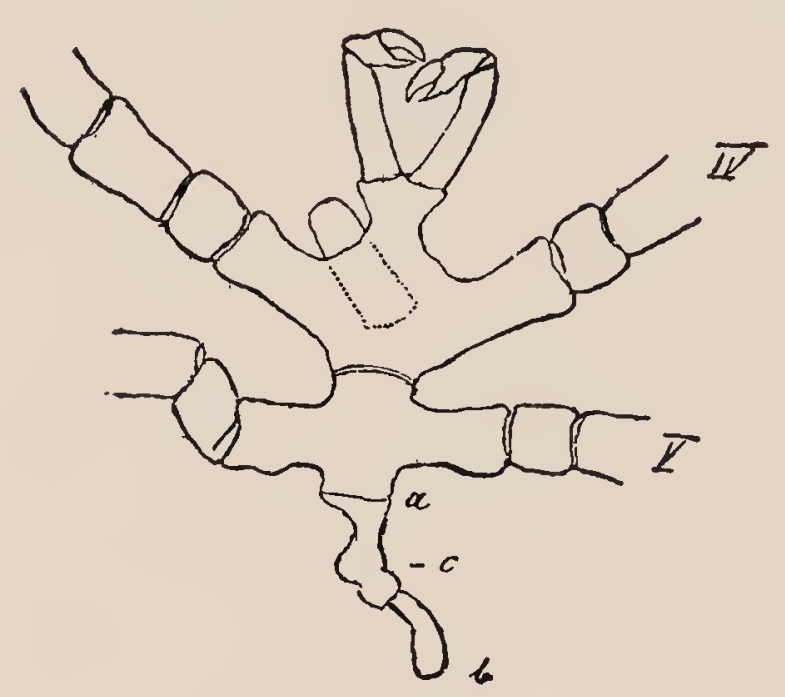

FIG. 96

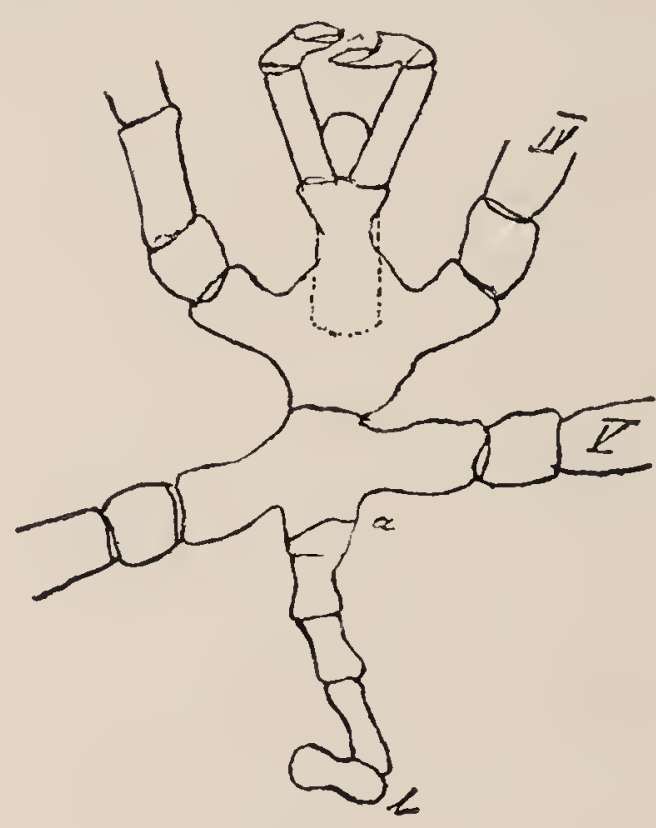

FIG. 97

I had the animal shown in Fig. 97 cut into serial sections, which I examined microscopically. The intestine had grown into the anterior portion of the regenerated piece. The tissues were, however, but little differentiated.

3. The regenerated pieces $a b$ of Figs. 96 and $97 \mathrm{did}$ not make their appearance gradually and then grow steadily larger, but they suddenly appeared with the size and differentiation shown in the picture, while on the previous day no regeneration had been visible. As each operated animal was kept in a separate dish upon which its history had been written, and as each animal was examined daily, we must conclude that the regeneration and growth of the new pieces occur slowly under the skin, and that at the next molting the regenerated piece becomes suddenly visible. I have

1 H⿵Eк, Archive de zoölogie expérimentelle, Vol. IX (1881). 
observed that the injured Pycnogonides continue to molt. I expect to resume these experiments and to fill out the gaps left in this study.

\section{ON THE THEORY OF REGENERATION}

1. Systematists have found it difficult to place the Pantopods in the natural system upon the basis of their morphological and developmental characteristics. It might be thought that under such conditions the consideration of the physiological behavior of the animals might offer advantages. On the basis of our observations on the regeneration of Pantopods it might appear as if the Pantopods were closely related to the Annelids; but this conclusion would be inaccurate, for, as is well known, a whole group of Annelids, the leeches, do not regenerate body segments which have been lost, even though transverse pieces cut from the leech may remain alive for more than a year. One would therefore have to reason that the Pantopods are more closely related to the Chætopods than the Hirudineæ, which would be absurd.

2. One frequently encounters the statement that the capacity for regeneration in animals decreases the higher animals stand in the natural system. This idea has been stated in a very definite form by Nussbaum: "The capacity of regeneration of organisms is proportional to their systematic position, as determined by their characteristics, and decreases from below upward." "This generalization goes too far, as can be seen from the facts mentioned above. Usually we find in every large group in the animal kingdom certain species with a greater, and others with a smaller power of regeneration. The salamander regenerates an amputated tail, inclusive of the spinal column and spinal cord, and this power of regeneration is almost as great as

1 NussBAum, Sitzungsberichte der Niederrheinischen Gesellschaft für Natur-u. Heilkunde, Bomn, November j, 1894. 
that observed among the Chætopods or Pantopods. On the other hand, the leech is not much more capable of regeneration after an injury to its body than the human being; both can only cover the amputated stump with skin. If one wishes to utilize the power of regeneration of animals for phylogenetic purposes, this can be done only for members belonging to one and the same morphological group. According to Sachs, only "the forms of the same group may be considered related to each other; they have nothing in common phylogenetically with the members of another group; every morphological group is, so to speak, a plant kingdom in itself." 1

But whether even within the same phylogenetic group, in the sense in which Sachs uses the term, the power of regeneration of a species is a simple function of its position in the group can at present, from lack of facts, not be decided. My experiments on the functions of the brain in worms showed that no parallelism exists between these functions and the systematic position of each species. Much less does such a parallelism exist in regard to the tropisms which can be altered comparatively easily through external conditions. But though it is not correct to say that the power of regeneration decreases the higher the animal stands in the system, it is perhaps true, that the number of species capable of complete regeneration is relatively greater in the groups of the Coelenterates and worms than in the groups of Arthropods and vertebrates.

3. In general, it will also be found correct that the power of regeneration is greater in the embryo than in the adult animal. The young larva of the frog regenerates an amputated leg, while this is not possible in the adult animal. Those who assume that the power of regeneration is the greater the lower the position of the animal in the natural

1 J. von SACHs, Flora, 1894, p. 219. 
system will find this behavior of the embryo in harmony with the "biogenetic law."

The explanation of the fact that the embryo possesses a greater capacity for regeneration than does the adult animal follows in a simple way from Sachs's theory of organization. Sachs assumes that the form of organs is determined by specific substances, and that we have just as many specific morphogenetic substances in a plant as there are different organs present in it; but these substances are by no means all preformed in the germ; they originate through chemical changes from the germ substance during the process of development.

If at first only those substances which lead to the formation of stems and roots are present, these, under the influence of external conditions, finally give rise little by little to another category of substances, which finally present themselves, in their purest form, in the male and female sexual cells. We can imagine this process as similar to the processes which follow one another in a chemical factory, where from the original raw material chemical compounds of the most varied kind gradually result, until finally the most valuable product, perhaps in an exceedingly small amount, is obtained in a pure form. ${ }^{1}$

In the sense of this theory, we must assume that there are at first present in the animal egg only specific ectoderm and entoderm substances, from which, through chemical changes during the process of development, such compounds originate as are specific for epithelial cells, liver cells, periost cells, etc. If now we ask how, according to this theory, e. g., an animal which shows complete power of regeneration (Planaria torva), differs from one which is able only to cover the amputation stump with skin (Hirudo), the answer will be, it seems to me, as follows: In the leech all the original embryonic material (the egg substance) is used up in the production of the specific organogenetic substances, while in

1.J. vos SACHS, Arbeiten des Botunischen Instituts in Wiurzburg, Vol. II, p. 457. 
Planaria not all the "raw material" is consumed; yet enough substance for the formation of epithelïum is still present in the leech for the repair of defects in the epithelium.

During embryonal development the more "raw material" is changed into the specitic organogenetic substances of the different organs, the larger the number of organs that are formed; in other words, the further the embryo progresses in the process of development. It is, therefore, easily intelligible why in some animals in which the "raw material" is present in only limited amounts regeneration is more complete in the earlier stages of the embryo than in the later stages. If the power of regeneration is dependent in this way upon the difference between the formation and consumption of the "raw material," it is not to be expected that the power of regeneration should be a function of the position of a species in the natural system. We can easily understand that this difference may be relatively great in one form, while in another form of the same group it drops to zero sooner or later during the embryonic development. We may expect, however, that we shall meet the latter state of affairs relatively more frequently in the more highly differentiated groups of Arthropods and vertebrates than in lower groups; which, indeed, seems to be the case. An idea of the rôle of the organogenetic substances in regeneration has been given by Sachs in his paper on "Stoff und Form der Pflanzenorgane." I have shown in several papers that the processes of regeneration, heteromorphosis and ontogenesis in animals, agree with the ideas of Sachs. ${ }^{1}$

1 Part I, p. 115; and On Some Facts and Principles of Physiological Morphology (Lectures Delivered at Woods Hole, 1893). 


\section{XIV}

\section{CONTRIBUTIONS TO THE BRAIN PHYSIOLOGY OF WORMS $^{1}$}

In his well-known work Ueber Entwicklungsgeschichte der Thicre K. E. von Baer asks how the anterior pair of ganglia of segmented animals should be designated.

Whether the first pair of ganglia of the segmented animals shall be called a brain or not depends entirely upon the significance which the word brain is given. It is certainly not the organ which we call the brain in vertebrates, for in them it is the anterior extremity of the neural tube, and this is lacking in the segmented animals. It is rather the foremost pair of the series of ganglia, and as the latter is to be compared with the spinal ganglia of the vertebrates the so-called brain of the segmented animals seems to correspond to the Gasserian ganglion of the vertebrates, ${ }^{2}$ for the latter also receives sensory impulses. . . . . If, however, one wishes to designate by the term brain not a definite organ, but .... that mass of nervous tissue which receives the sensory impulses, then one can, of course, say that insects possess a brain. Only one must keep the meaning of this term in mind.

He who seeks a definition for the word brain will find the necessary directions in von Baer's remarks. Steiner ${ }^{3}$ considers it the problem of the physiologist to find such a definition, and has come, apparently without knowledge of the remarks of von Baer, to a different definition which reads as follows:

The brain is characterized by being the general center of locomotion in connection with the activities of at least one of the higher sensory nerves. . . . Besides its simplicity this definition

1 Pfügers Archiv, Vol. LVI (1894), p. 247.

2 Vol. I (1828), pp. $234 \mathrm{ff}$.

${ }^{3}$ Sitzungsberichte der Berliner Akademie der Wissenschuften, 1890. 
has the further advantage of being satisfied by a single experiment, in so far as, of the two elements of which the definition is composed, the one element is always given anatomically. This is the higher sensory nerve, the presence of which guarantees its function. The only experiment which it is necessary to perform has to prove that the general center of locomotion is also present beside the sensory apparatus. This proof is furnished when the unilateral removal of the central nervous portion in question so alters the direction of the movement of the animal that it no longer moves in a straight line but in a circle-a phenomenon which is generally designated by the term "forced movements."

This definition of Steiner leads to two new conclusions: first, that the cerebrum in human beings does not belong to the brain since, as is well known, unilateral removal does not bring about forced movements. Stteiner himself realizes this, for he has found, aided by his definition, that the octopus "has a cerebrum but no brain." "To have no brain and yet a cerebrum seems strange and even paradoxical, probably only, however, because we have not until now encountered such a case." The second necessary conclusion from Steiner's definition is that the ear is a brain. This conclusion has not been drawn by Steiner himself, but is unavoidable. For, first, unilateral extirpation of the ear brings about forced movements, and, secondly, the auditory nerve is one of the higher sensory nerves. Steiner further points out that bilateral destruction of an organ, the unilateral destruction of which brings about circus motions, renders impossible spontaneous progressive movements. Forced movements can be brought about in the shark from the medulla oblongata, and here is located also, according to Steiner, "the general center of locomotion" of this animal. Steiner has himself shown, however, that a shark still moves spontaneously after the loss of the entire medulla oblongata."

This "center of locomotion" is said by Steiner to be located in the medulla oblongata in the frog also, but 
Schrader has found that a frog is possessed of an irresistible impulse to move after losing this center. ${ }^{1}$

The simplest facts of comparative physiology show moreover that the power of progressive movement is possessed also by such organisms which have no brain whatever, $i$. e., the swarm spores of Algie. It is, in my opinion, not the problem of physiology to find a definition for an organ but to discover the functions of a given organ.

From this standpoint I wish to make in the following pages some contributions to the brain physiology of worms. I understand in this paper by the term brain, as is customary, the ganglia lying at the oral end of these animals. Brain

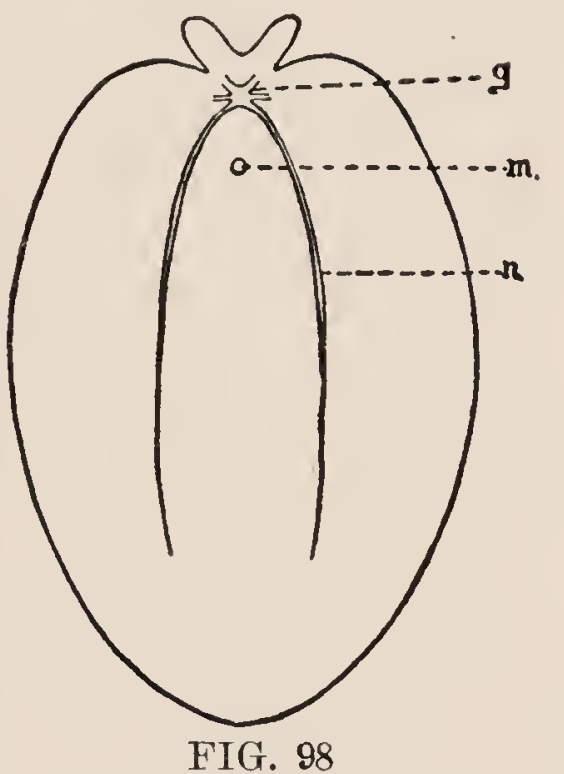
physiology has shown that for the higher animals the biological character of a species, that is, the sum total of those reactions of a species which are determined by the external surroundings, depends chiefly upon the brain. I was especially interested in determining whether the rudimentary brain of such low animals as the worms has a similar significance. The experiments which I wish to report have been made at long intervals, some in Naples in 1889, some in Woods Hole in 1893.

II

\section{EXPERIMENTS ON THYSANOZOON BROCCHII}

1. Thysanozoon is an elliptically shaped marine Planarian (Fig. 98, according to Lang), which is from one to three $\mathrm{cm}$. long, and almost as broad. The brain $g$ of the animal, an unpaired organ, is situated at the anterior extremity of the body, which latter can be recognized without difficulty by

1 Schrader, Pflügers Archiv, Vol. XLI. 
its possession of two tentacles (Fig. 98). From the posterior side of the brain arise the two large nerves (n, Fig. 98), which traverse the entire length of the animal. A number of other nerves also run to the brain. The nerves contain separate ganglion cells, a characteristic which, as is well known, is found also in certain peripheral nerves in the higher animals. The nerves form a plexus at the periphery. ${ }^{1}$

The central nervous system of this animal therefore consists mainly of the ganglion situated at the anterior extremity of the animal. As is the case with all Planarians, Thysanozoon creeps over the walls of the aquarium or along the surface of the water. It differs in its movements from the fresh-water Planarians only in so far as it is able to execute actual swimming movements. In swimming the animal executes pendulum-like movements with the lateral portions of its body as does a butterfly with its wings.

If a Thysanozoon is cut across transversely into two halves, while the animal is moving at the surface of the water, the posterior aboral half at once falls to the bottom like a dead mass, while the oral piece which contains the brain continues to move along quietly. If the cut is made rapidly and with a sharp pair of scissors, the behavior of the oral piece gives no indications of such reactions as accompany the sensation of pain in higher animals. If the animal is divided with a sharp knife while it is creeping over a glass plate, we notice the same phenomenon: the oral piece continues its motion undisturbed while the progressive movements of the posterior piece cease at once. It happens occasionally that the transverse division of a Thysanozoon causes the oral piece to execute more lively and rapid movements. That the progressive movements of this animal are indeed a function of the brain is shown particularly well when only the exceedingly small piece which contains the 
brain (Fig. 99) at the anterior end of a large (about $3 \mathrm{~cm}$. long) Thysanozoon is cut off. The very small piece lying anteriorly then continues to creep or swim while the comparatively enormously large body executes no further progressive movements. The spontaneity of progressive movement in Thysanozoon is therefore " function of the brain.

Both pieces of a divided Thysanozoon continue to live and regenerate the missing portions. Only the oral piece regenerates more rapidly than the aboral, which has to form a head. I have not studied whether the latter forms a new brain. I kept such pieces alive for four months. The spontaneity of the aboral piece never returned, while the spontaneity of the oral piece persisted.

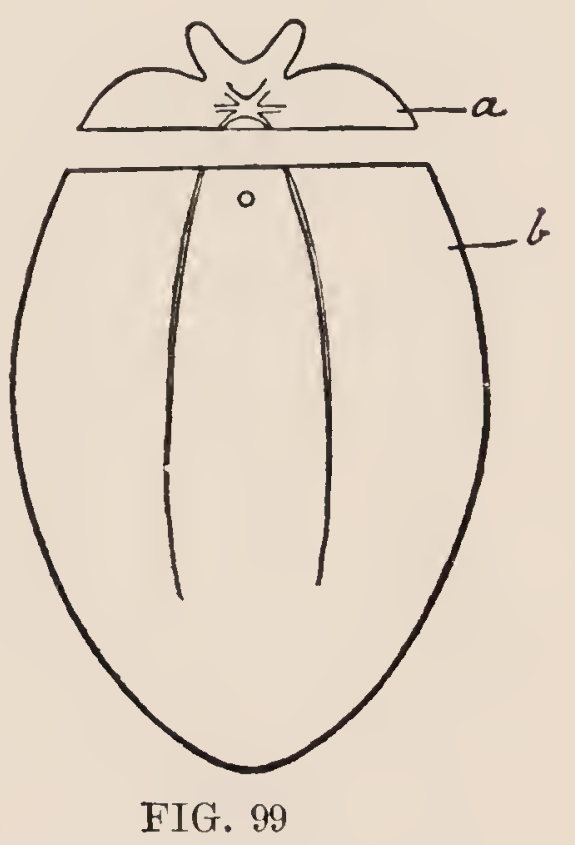

2. The beheaded frog will remain upon its back when the cut lies behind the medulla oblongata; when the cut lies in front of the medulla the frog will not remain upon its back. We assume in this case that the geotropic functions of the ear compel the frog to resume the normal orientation. It is probable that the tactile stimuli also act in such a way that they compel the frog to bring the soles of its feet in contact with the surface of solid bodies, or to allow the weight of its body to press upon the nerve endings in these portions of the skin.

I designated the fact that an animal is compelled to orient its body in a definite way toward the surface of solid bodies as stereotropism. Geotropism cannot be demonstrated in Thysanozoon as the animal assumes any orientation toward the center of gravity for a long time. Stereotropism is, however, present as the animal is compelled to bring its ventral surface in contact with solid bodies, or to allow its 
body to come to rest upon its ventral surface. We cannot compel the animal to bring its back in contact with solid bodies, and at the same time expose its ventral surface to the water. ${ }^{1}$

The question now arises whether these phenomena of

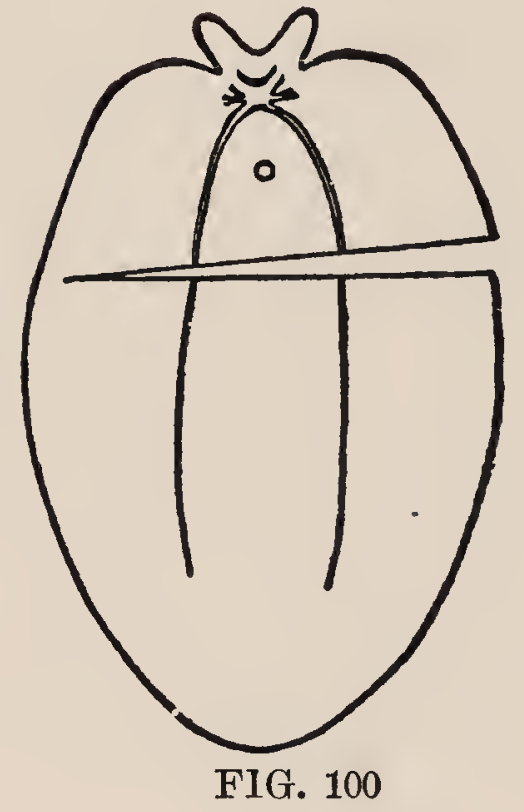
orientation are a function of the brain as are the spontaneous progressive movements. Strange to say this is not the case. The brainless Thysanozoon returns to the ventral position when it is laid upon its back, only the reaction occurs more slowly than in the normal animal, or in that portion of the animal containing the brain. Reactions to light could not be demonstrated.

3. If, instead of making a complete transverse section of the animal, only the longitudinal nerves are cut, and the two pieces are left united with each other by a very thin bridge of protoplasm at one side (Fig. 100), the aboral piece is not innervated directly by the nerves from the brain. A conduction of the impulse by way of the lateral nerve plexus is, of course, still possible.

When, immediately, after the operation, I laid such an animal upon the bottom of the aquarium the oral piece at once began to move, while the aboral piece tried to attach itself to the bottom. It responded, however, to the pull which the oral piece exerted upon it, and took part in a perfectly co-ordinated manner in the progressive movements, as if no interruption had occurred. After some time the oral piece turned about, crept over the back of the aboral piece whereby the latter was dragged along passively, and laid

1 The righting of starfish which have been laid upon their backs is also only a case of stereotropism, and has nothing to do with the effects of gravity. 
upon its back. The posterior piece at once resumed the ventral position, however, and then moved actively in the same direction as the oral piece. Changes in movement, therefore, were inaugurated only by the oral piece which contained the brain and were never communicated directly to the aboral piece. When, however, the oral piece moved for some time in the same direction and with the same velocity, the same movement soon took place in the aboral piece also. The aboral piece therefore, behaved not entirely as a piece without the brain, as it was still capable of progressive movement, but not as a normal Thysanozoon either, as it had lost its spontaneity. This becomes still clearer from the following observations:

I threw the animal into a basin of water. Both pieces executed energetic, synchronous swimming movements. The oral piece soon reached the vertical glass wall of the aquarium. In consequence of a change in the direction of the movement of the anterior piece, the connecting bridge between the two halves of the animal was twisted and the aboral piece came in contact with the glass wall with its back, while the ventral surface of the same was turned toward the water. The posterior piece now executed swimming motions and so followed the creeping movements of the oral piece. That when movement is constant the posterior piece takes an active part in the progressive movement, and is not merely dragged along passively, is further shown by the fact that it often crept with its free edge upon the back of the oral animal, especially when the latter suddenly moved more slowly.

The experiments detailed thus far show that a brainless piece of Thysanozoon no longer moves spontaneously, that is to say without an appreciable external stimulus. I did not succeed in bringing about progressive movements in a brainless Thysanozoon even by stimulating it. If the animal is 
touched a local contraction occurs at the stimulated point, but no progressive movements.

4. If a lateral piece $a b$ is cut from an animal parallel to the median plane (Fig. 101), a contraction of the wound results which may be so great that the piece rolls up into a spiral.

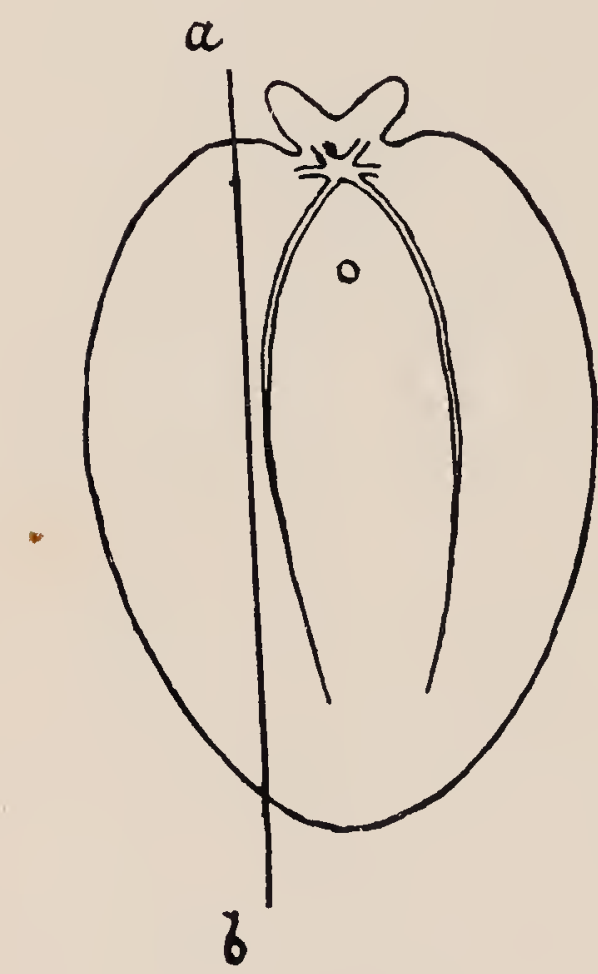

FIG. 101

(This contraction of the wound-edges occurs no matter what the position of the cut.) The lateral piece $a \bar{b}$, which contains no brain, executes no progressive movements. If, however, a contraction of the wound-edge occurs in the other piece, so that it is rolled into a spiral, the progressive movements no longer occur in a straight line but in a circle. I never succeeded in bringing about circular movements through unilateral destruction of the brain in Thysanozoon.

II. EXPERIMENTS ON PLANARIA TORVA

1. The brain and nervous system of the fresh water Planarian (Fig. 102, according to Jijima) are so analogous to those of the marine Planarian, that it is unnecessary for our purposes to give a separate description of them. The most important difference exists perhaps in the fact that the two longitudinal nerves contain certain collections of ganglion cells. One might think that the brain

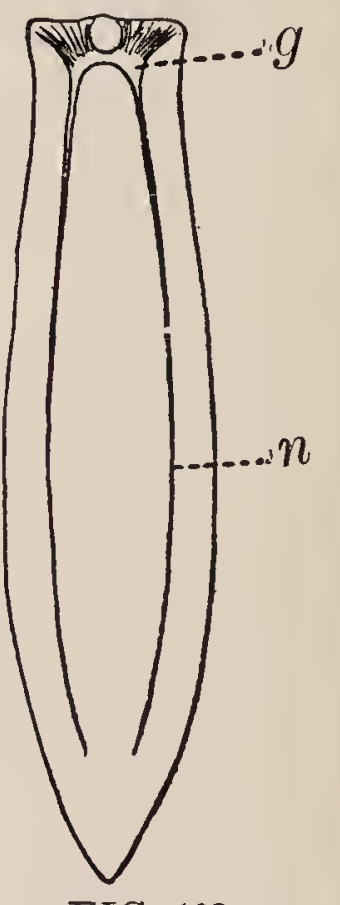

FIG. 102 function of the fresh-water Planarians might also be analogous to those of the Polyclads. That is, however, not the case. We experience here again what I have pointed out repeatedly in my papers on the lower animals: that animals which are very closely related morphologically may show the greatest 
differences in their physiological reactions. If a fresh-water Planarian is cut in two the aboral piece which contains no brain creeps about in just as lively a manner as the oral half. The spontaneity of the progressive movements in Planaria torva is therefore in no way a function of the brain. Every piece of the animal, not too small, possesses spontaneity. The decapitated animals creep with the oral end directed forward, as do normal animals.

2. In a previous paper I have described the behavior of Planaria torva toward light. The animals are chiefly photokinetic, that is to say, changes in the intensity of the light alter their morements. If the animals are suddenly brought from darkness into light they begin to move. During the first few moments the direction of the movements is also influenced by the light. The animals move as do negatively heliotropic animals to the room side of the vessel, but they do not collect here as do negatively heliotropic animals, but distribute themselves in all directions, and now begin to move in every direction, to come to rest finally in that region of the vessel which is more weakly illuminated than its surrounding.

One receives the impression therefore that an increase in the intensity of the light causes the animals to move, while a decrease in the intensity of the light causes them to come to rest. For this reason one finds the animals through the day collected in relatively dark places in the vessel, or on the under-surface of stones. I suspect that the animals begin to move anew at night, and then at the approach of day again collect in relatively dark places. I repeatedly covered onehalf of the glass vessel in which I kept the Planarians with black paper in the morning. No change occurred through the day. On the next morning, however, I found all the animals under the covered portion of the aquarium. This could be interpreted only as showing that the animals crept 
about in the vessel during the night, and came to rest in the morning in the darkest regions.

These animals possess at the oral pole not only a brain but also comparatively well-developed eyes. I decided to test whether a decapitated Planarian still shows the same reactions toward light as normal Planarians, in spite of the loss of brain and eyes. This is true in a most surprising way. About sixty specimens of Planaria torva were cut across transversely, close behind the brain and the eyes, in the evening. All the pieces were put into a vessel having vertical sides and half covered with black paper. On the next morning nearly all the posterior pieces as well as the oral pieces were found in the covered part of the aquarium. They were fairly uniformly distributed here. A few were found in the uncovered portion of the vessel, but among these there were head pieces as well as aboral pieces crowded together in one corner in the room side of the dish. In this corner the intensity of the light was relatively low. In repeating this experiment with normal animals I obtained the same results as with the decapitated animals. When the decapitated animals had collected in the covered portion of the ressel and had become perfectly quiet, their quiet was quickly disturbed when the dark paper was suddenly removed without jarring the vessel. The animals began to move, crept at first toward the room side, and finally collected again in regions where the light was least intense. This reaction also occurred as in normal animals, with this difference, however, that the reaction time of the brainless animals to changes in the intensity of the light was greater than in normal animals. In the animals possessing a brain and eyes the reaction began about one minute after light struck them; in the brainless pieces after about five minutes. In these experiments only diffuse daylight was used as a stimulus.

I have pointed out before that when uninjured Planarians 
are put into a vessel having a cylindrical form, they do not collect as do purely heliotropic animals on the window or room side of the vessel but on the right and left sides of the same. The Planarians from which the head together with the brain and the eyes have been removed behave in exactly the same way. All these experiments are successful as early as the day after the operation. Only perfectly fresh material must be used for these experiments. In warm weather the posterior pieces regenerate a new head with eyes and probably a brain after a week.

3. It was formerly the custom to conclude that an animal possessed eyes when it reacted to light. And since no one doubted that the reaction to light was a reflex movement it was assumed that such animals possessed a central nervous system also. When I found that the orientation of animals toward light is determined by the same conditions as the orientation of plant organs toward the same stimulus I drew the self-evident conclusion that the orientation of the animals toward the light could not possibly rest upon characteristics which are possessed only by the eyes or only by the brain, as plants do not possess such organs. The sensitiveness of the eye to light must rather rest upon the fact that the eyes have a condition in common with heliotropic plants, namely, elements which suffer some change under the influence of the light. For the rest, however, these elements need not be either morphologically or chemically identical in the different organisms. An analogous conclusion may be drawn for the brain. When an animal with a brain shows the same reaction as a plant which possesses no brain it follows that the reaction toward light is not the result of a "specific energy" of the brain, but that the brain in that case only performs a function which is possible in plants without nerves. This function need be nothing else than the conduction of the light stimulus, which, as is well known, occurs 
in plants also, but in the latter case through different mechanisms. These facts which I considered as self-evident have been misunderstood by one author and have been falsely represented, as though I had asserted that brain and eyes are superfluous.

From what I said it also followed that in animals which possess eyes the sensitiveness to light need not by any means be limited to the eyes. Photosensitive elements may be found also in other portions of the surface of the body. Graber has in fact already reported experiments on Tritons and angleworms which he claims point in the same direction. It could, moreover, be foreseen that an effect of light might perhaps be possible in many animals without the existence of a true reflex arc, for I found in Ciona intestinalis that a typical reflex phenomenon continues to exist in this animal after destruction of the central nervous system. There was finally the possibility also that both conditions might be found together in the same animal. The latter is the case in Planaria torva, determined to be such by Dr. Wheeler.

4. After what has been said it is scarcely necessary to emphasize the fact that brainless pieces of Planaria torva will not assume the dorsal position any more than will uninjured animals. All my experiments to bring about forced movements in these animals through unilateral destruction of the brain remain as fruitless as in Thysanozoon.

\section{EXPERIMENTS IN CEREBRATULUS MARGINATUS}

The Nemertines possess a more highly developed brain than do the Planarians. The brain is larger and shows also a greater subdivision into smaller parts. It also is continued into two lateral nervous cords. The latter contain "a superficial covering of ganglion cells which may give rise to ganglion-like swellings at the points where the nerves branch."

${ }^{1}$ Clauds, Lehrbuch der Zoologie, 4. Aufl., p. 336. 
The specimens of Cerebratulus which I used in my experiments were over $50 \mathrm{~cm}$. long and almost as thick as a finger. The layman would readily have believed that he was dealing with an eel instead of with a worm. The animal lives in the sand. If it is laid upon the sand-covered bottom of the aquarium it soon buries itself in the sand. If the head is amputated from such an animal the head-piece continues to bury itself in the sand when it is not too short. The body, on the other hand, does not make a single attempt to bury itself in the sand.

\section{EXPERIMENTS ON ANNELIDS}

The Annelids possess besides a fairly complex brain a chain of ganglia which transverse the entire length of the body. We have the experiments of B. Friedländer and of Graber on the function of the central nervous system of the Annelids.

Friedländer ${ }^{1}$ amputated the anterior and posterior segments from angleworms.

The latter conduct themselves, to put it shortly, as normal animals: they soon bury themselves in the earth. Not so the beheaded worms. Immediately after the operation they execute violent winding motions, perhaps creep about for some time, but usually come to rest after a short time, and can now remain quietly upon moist earth covered with moist filter paper, for days and weeks without, apparently, making any autonomous movements after the wounds have healed. Every stimulus however soon awakens them from their passive condition. They then move about energetically, even creep some distance, but soon fall back into their original lethargy.

The second series of experiments of Friedländer consisted in excising a small ( 5 to $10 \mathrm{~mm}$. long) piece from the abdominal nerve-cord of angleworms. Friedländer had expected that the two portions of the worm lying anterior and posterior to the operated point would conduct themselves physiologically during

1 Biologisches Centralblatt, Vol. VIII. 
locomotion as two individuals, or perhaps that the posterior portion would simply be dragged along passively. Neither, however, occurs. The animals which lack entirely a portion of the central nervous system creep about as normal animals, except for a slight variation which will be described later. A contraction of the longitudinal muscles begins in the anterior segments which passes posteriorly, reaches the operated point, jumps over it, and continues behind it, so that the movements of both parts are co-ordinated in exactly the same way as in the normal animal.

To explain this remarkable phenomenon Friedländer assumes that a "longitudinal pull" is exerted upon the posterior segments through the contraction of the anterior segments. "This acts as a stimulus to the stretched portions of the abdominal nerve cord, and the reflex brought about thereby consists of a contraction of the longitudinal muscles of the stretched segments." The longitudinal stretching of the skin (not of the abdominal nerve cord) would therefore liberate reflexly a longitudinal contraction. The correctness of this idea was proved by the following experiment. An angleworm is cut in two in the middle and both pieces are sewed together in such a way that they are connected by a thread about $1 \mathrm{~cm}$. long. The pieces when connected in this way by means of a thread execute co-ordinated movements.

Graber tested the statement of Hofmeister and Darwin that the anterior end of the body of the angleworm is sensitive to light. ${ }^{1}$ He amputated the anterior segments of angleworms, and found that the brainless pieces were still sensitive to light. The reaction of the angleworm to light is therefore no function of the brain alone. My own experiments consisted in amplifying these facts in some directions.

\section{EXPERIMENTS ON NEREIS}

If a Nereis is cut into several pieces, only the oral piece retains the power of burying itself in the sand. Earlier

1 Grundlinien zur Erforschung des Helligkeits- und Farbensinnes der Thiere, Prague (1884), p. 290. 
experiments had led me to suspect that this "spontaneous" or "instinctive" burial was only a reflex called forth through the stimulus of contact with sand. I tried whether it would not be possible under certain conditions to demonstrate the same reflex, even in brainless pieces. I laid such a brainless piece upon the sand; as usual it remained quiet. I now carefully covered the anterior end of this piece with sand. The rest of the animal soon began to execute the typical movements which are necessary to bring about the burial of the animal in the sand. At the same time the glands at the foot end of the animal began to secrete the sticky substance which cements together the grains of sand and renders solid the wall of the tube in which the animal lives. This secretion is a constant accompaniment of the burrowing of the animal. It is the same secretion which in other worms leads to the formation of a tube. The animal did not however succeed in burying itself, and so it soon began its movements anew.

Spontaneous progressive movements were executed only by that piece which contained the brain. Yet weak stimuli, such as the shaking of the aquarium in passing through the room, sufficed to bring about progressive movements in the posterior piece. None of the pieces would remain on their backs when turned over. When I attempted to keep a brainless piece forcibly in this position it made great attempts to return to the ventral position. In all the experiments which have been described the size of the piece is not immaterial: the more segments it contains the more definite are its reactions. I tried finally to determine whether all the ganglia even the most posterior are able to bring about the same winding and bending which is characteristic of the injured worm. That is indeed the case.

\section{EXPERIMENTS ON LUMBRICUS FCTIDUS}

I wished to determine whether angleworms are heliotropic or photokinetic, and whether the decapitated animals show 
the same relation to light in every particular as do the animals with the brain. If Lumbricus foetidus is introduced into a transparent closed vessel it is noticed first of all that the animals are strongly stereotropic. As soon as they reach the concavity of a corner or a groove they crawl along it and do not leave it. Secondly it can be shown that they are photokinetic. They come to rest in those regions which are more weakly illuminated than the surrounding areas. The direction of the rays of light is of little consequence. It seems also as if, when one or more animals have come to rest at any point, the others also come to rest at the same place more readily. This looks as though the animals were "social." This may be attributed to a chemotropic irritability.

It is noteworthy that the less refrangible rays which pass through red glass are less active for photokinetic animals than the more strongly refrangible rays which go through blue glass. The angleworms come to rest sooner under red glass than under blue glass. (We can speak of a "preference" for red light for this case just as little as in the case of heliotropic animals.) Decapitated Lumbrici fœtidi all show the same stereotropism as normal animals. When they reached the concave side of the corner of a vessel they did not readily leave the corner again. The decapitated animals also came to rest as did the normal animals in those regions where the light was least intense, while an increase in the intensity of the light stimulated them to movement. It could also be shown that the light which passes through blue glass acted in this regard as light of a greater intensity than that which passes through red glass.

In all these experiments the decapitated pieces crept about with their tail end forward as well as with the oral end forward. I noticed repeatedly a fact which shows that even the extreme caudal end of the angleworm is sensitive to light. For when the caudal end suddenly entered an illuminated 
area from one which was protected from the light it at once turned about.

Strange to say the reaction-time to light is not markedly greater in decapitated angleworms than in normal animals. The experimental animals were contained in a dark box in which they could without being jaried be suddenly exposed to diffuse daylight. Three to eighteen seconds after the entrance of the light the decapitated angleworms first began to move. It took about the same time in normal worms.

Lumbricus fotidus lives in decaying straw and manure, and it can readily be assumed that the chemical nature of certain substances contained in the straw and manure holds the animals - that in other words they are positively chemotropic to the substances. I could readily show that when one-half of the bottom of a box was covered with white moist filter-paper and the other half with a thin layer of decaying straw, the normal worms which were laid upon the filterpaper were soon all collected upon the manure. The posterior pieces of transversely severed worms behave in exactly the same way. When they were laid upon the filter-paper they were not directly attracted by the odorous substances contained in the manure. As soon, however, as they came in contact with the manure in their progressive movements they crept upon it, and once upon it they did not leave it again. In this way it soon happened that all the brainless worms were collected, without exception, upon the manure. When they were laid upon a heap of decaying straw, they soon buried themselves in it. That was not alone the effect of the light, for the reaction occurred also in the dark.

\section{EXPERIMENTS ON LEECHES}

If a leech is cut in two transversely the two pieces show entirely different reactions. The wound soon heals and the pieces may live a year or more withont however, as is well 
known, any regeneration occurring. Both pieces can execute swimming motions: in the case of the posterior piece the oral end leads. Both pieces attach themselves by means of their suction disks. At times I also saw the posterior piece execute lively progressive movements, without however being able to discover an external stimulus for this movement. Both pieces returned to the ventral position when they were laid upon their backs. For the rest however the two pieces behaved differently. While I frequently found the anterior piece attached to the vertical glass sides of the aquarium, I found the posterior piece attached to the bottom. I suspected that the attachment of the suction disk came to pass reflexively, in consequence of the pressure or the friction to which the skin of the suction disk is exposed when it is allowed to come in contact with the bottom. It was therefore to be expected that it should be possible to compel the animal to attach itself to any desired point by pressing the suction disk against it. The experiment succeeds very readily with the posterior piece of a transversely divided animal, when it is gently pressed against the desired spot by means of a brush. The anterior piece however behaves entirely differently when the same experiment is made with it. It turns itself in an apparently aggressive manner against the brush so that it is impossibe to press the suction disk against the wall.

If the posterior piece is laid upon its back, it begins to execute swimming motions by means of which it brings itself back into the ventral position. The anterior piece returns to the ventral position by turning over. The tendency to execute swimming motions is much more marked in the posterior than in the anterior piece. If the leech is divided in such a way that the two pieces are still connected by a small piece of skin, they at times cxecute co-ordinated progressive movements, as in Friedländer's experiment. More 
frequently however one observes that the posterior piece apparently begins its swimming motions spontaneously, and pushes before it the anterior piece which contracts and ruffles itself.

\section{III}

1. Our observations therefore show that when a worm is cut through transversely that piece which contains the brain retains to a greater degree, generally speaking, the biological or psychic character of the species than the brainless piece, even when the latter far exceeds the anterior piece in mass. The difference which the oral and aboral pieces show in this regard is different in different species of worms. In Thysanozoon this difference is marked, also in leeches and in Cerebratulus, while in Lumbricus and especially in Planaria torva ${ }^{1}$ it is less. It is however questionable whether this difference is chiefly determined by the brain. For we do not know how far the specific irritability of the individual peripheral elements of the oral pole has to do with it.

2. The latter thought may go too far for many readers. But it seems to me that we are too much inclined to seek the "irritable structure" which determines the reaction of an animal exclusively in the central nervous system, while frequently a more careful analysis of the phenomena by no means compels such a conclusion. The Ascidians are the simplest reflex animals. The central nervous system is reduced to a single ganglion which receives sensory fibers from the surface and sends motor fibers to the muscles. If the skin of the animal is touched, the muscles contract, and the oral and aboral openings of the animal close. Under these circumstances the stimulus passes from the touched spot to the ganglion and from here to the muscles. Tke ganglion can be readily extirpated in transparent forms such

1 In this form Bardeen has recently found that the so-called longitud nal nerves resemble more closely the oral ganglion in their histological structua: 
as Ciona intestinalis. After the loss of the ganglion; however, the animal reacts in the same way when touched as before. Only one condition is different. The intensity of the stimulus which is necessary to bring about a reaction in the brainless Ciona is much stronger than in the intact animal. This indicates that the mechanism is different in the two cases. In the normal animals the sensory nerves are stimulated and the stimulus passes through the ganglion to the muscles. In the brainless animal the muscles at the irritated point are possibly stimulated directly. The contraction of these muscles is then the cause of the contraction of the neighboring muscles and so on. ${ }^{1} \quad$ Under these circumstances it is more than a mere possibility that in the normal Ciona also the characteristic reaction when touched is determined not by the brain, but that the brain serves the purpose in this case of a better and more rapid conductor of the stimulus. The nature of the reaction might rather be chiefly determined by the arrangement of the muscles.

The heliotropic phenomena of animals are identical in all respects with those of plants. The latter have no central nervous system and the remarkable nature of these reactions is therefore not necessarily determined in animals also by the specific characteristics of their reflex centers. It is much more probable that the nervous system plays in this only the rôle of a conductor of stimuli, while the actual character of the process is determined by the following conditions: (1) The shape of the body and the topographical distribution of irritability corresponding with it. (2) The changes brought about by the light in the illuminated tissues: (3) The conduction of the stimulus to the contractile tissues. (4) The arrangement and structure of the latter.

Examples of the same sort are the observations described

$1 \mathrm{It}$ is however possible that the stimulus is conducted to the individual muscle fibers through nerves. [1903] 
in this paper on stereotropism in brainless Planarians, and the reaction of brainless animals toward light.

3. Even though the brain, or more accurately that part of the body containing the brain, determines in the main the biological or psychic reactions of worms which are typical for each species in the same way as in higher animals, there nevertheless exists a specific difference between the brain of worms and that of many of the higher animals. The worms lack associative memory and consequently also consciousness, which is only a function of the former. By associative memory we understand that arrangement of the brain by virtue of which a stimulus brings about not only the effects corresponding with its nature and the specific structure of the irritable tissue, but also the stimulating effects of other causes which at a previous time once affected the organism at the same or almost the same time with the stimulus. No trace of such an associative effect of stimuli can be proved to exist in worms, and consequently also no trace of consciousness.

One might go farther and speak of memory, when the effect of a stimulus depends upon previous effects of stimulation at all. Under these circumstances, for example, we would have to designate it as memory when a plant which was originally cultivated in the tropics does not withstand low temperatures as well as a plant of the same species cultivated in the North. We would also have to call it memory when a Medusa of the temperate zone is moved to the regions of the midnight sun and here continues its depth migrations in a period corresponding with the changes of day and night in its home. No objection could be made to this, only I do not believe that this kind of memory can be looked upon as a lower form of associative memory. And I am convinced that it is something entirely different from associative memory. When the plant which has been cultivated in the South does not do as well in the North as the same species of plant 
which has been cultivated in the North it is to be attributed to a difference in the constitution of the tissues of the two plants, possibly to a difference in the amount of water contained in the two tissues. The periodic depth migration of the Medusa are, as I believe, brought about through a periodic change resulting from internal causes in the amount of water contained in the animal, which, in the temperate zones, corresponds in its period with the change of day and night. (I suspect that the light brings about a change in the amount of water contained in the animal in one sense, while darkness brings it about in the opposite sense.) When the Medusa is then transferred to the North there is no occasion for a change in the period. In associative memory, on the other hand, we have to deal, it seems to me, with a definite mechanical arrangement which, from experiment and pathological experience, has to be sought in the brain and which is present only in certain animals, while it is missing in others. Correspondingly consciousness is present also only in certain animals, and in these only after a certain stage in embryonal development has been reached. To claim, as does one English author, that a "subconsciousness" exists in the egg, I consider just as wrong as though one would say that a subphonograph exists in a drop of water. The Darwinian habit of seeing transitions everywhere becomes erroneous when it attempts to take into consideration machines which yield qualified energy. And we have to do with such machines in the case of associative memory, as well as in many other physiological apparatus. ${ }^{1}$

4. If, therefore, a decided difference exists between many vertebrates and the worms (and other lower animals) so far as associated memory and consciousness are concerned,

1 In many respects my views coincide with those expressed by DrIEsch in his excellent booklet Die Brologie als selbstständige Wissenschaft. 
only a quantitative difference exists so far as spontaneity is concerned; when we designate as spontaneous those changes in an animal which are the result of internal, or more correctly, which occur without demonstrable external stimuli. As a matter of fact, many changes brought about through external stimuli will seem spontaneous to us because the external stimulus escapes our observation. We have to distinguish between conscious spontaneous changes (the true will-action in which the idea of the coming change precedes the latter) and simple spontaneous changes in which internal causes determine the latter without processes of consciousness being present. In the case of worms, of course, we can speak only of the latter forms of spontaneity. In Thysanozoon this spontaneity seems to be exclusively a function of the brain. In Planaria torva, on the other hand, this is not so distinctly the case. When compared with the number of reactions to external stimuli the number of the spontaneous movements of worms is small. Only where associative memory is present do the spontaneous changes step into the foreground numerically.

5. Whether the sensations of pleasure and pain are possible without consciousness cannot be decided absolutely. If it is permissible to consider the reactions of a frog when its skin is touched with acid, or the bending of a worm when one steps on it, as an expression of a sensation of pain, ${ }^{1}$ our experiments show that all pieces of a worm are capable of the sensation of pain. It is worthy of notice, however, that the reactions pointing to sensations of pain are weaker in Planarians than in Annelids, or may be lacking altogether.

6. One might be led to believe that the reflex motions in higher animals depend to a higher degree upon the

$1 \mathrm{~W}$. W. Norman has since shown that this is not permissible. The problem is more fully discussed in my book on the Comparative Physiology of the Brain. [1903] 
structure of the central nervous system than in lower animals. The fact that Planarians continue to react upon light if their brain is removed, or that Ciona continues to show its characteristic reaction after the loss of its ganglion seems to suggest such an idea. But it would be false, nevertheless. The contraction of the iris of the eye, if stronger light falls into the latter, is a typical reflex action, called forth through the effect of the light upon the retina. If, however, the reflex center is destroyed or the iris cut out, an increase of the intensity of the light which strikes the iris continues to cause a contraction of the iris. This fact is known for frogs and eels, and I have observed it in sharks. It is probably true for mammalians also. The reflex act therefore may serve here, as in Planarians, for the greater conduction of stimuli.

When a dog, whose spinal cord has been cut, is lifted so that its body hangs down vertically, a peculiar fact can be observed, as Goltz has shown. The legs are thrown into pendulum-like motions resembling walking motions. These motions are produced by the passive stretching to which the skin on the ventral side of the hip-joints is subjected through the weight of the legs. These pendulum-like motions are comparable to the reflectory contraction of the longitudinal muscles of the earthworm when its skin is stretched. This reflex would suffice to call forth co-ordinated walking motions in the dog whose spinal cord is severed, if such a dog were only able to stand on its legs. The walking motions of the anterior legs would produce periodically the stretching of the skin which is required for the locomotion of the posterior legs. The difference in the behavior of a dog with severed spinal cord and an earthworm with severed ganglionic chain in regard to co-ordinated locomotion is therefore less determined by the differences in the function of their central nervous system than by differences in the structure of their 
peripheral organs of locomotion. If the dog possessed, in the place of its high legs, short bristles like the earthworm, the dog with severed spinal cord would continue to make co-ordinated progressive motions as the earthworm in Friedländer's experiment. 


\section{THE PHYSIOLOGICAL EFFECTS OF LACK OF OXYGEN ${ }^{1}$}

\section{INTRODUCTION}

More than a century ago Spallanzani published his observations on the effect of stagnant air upon animals and plants. Spallanzani introduced organisms into hermetically sealed vessels of various sizes (which were, however, filled with air), and found that the smaller the vessel, the earlier did all life cease to exist in it. He also observed that different organisms show an unequal resistance to lack of air. As the most remarkable case he cites Anguillula aceti: "They live and multiply prodigiously where the volume of air does not exceed three inches; and die in several days only, when confined in a tube where the vacuum is less than an inch." "2

The further experiments on the same subject have fully confirmed the observations of Spallanzani. Bunge, in his well-known treatise on the respiration of mud-dwelling organisms, concludes that apparently "all transitional stages exist in the animal kingdom from the anaërobic unicellular organisms up to the most highly organized animals with a most energetic demand for oxygen." 3

A series of brilliant observations have served to elucidate the chemical side of these phenomena. It is an established fact that carbon dioxide can be produced in an organism without the presence of oxygen. Hermann has shown that the excised muscle of the frog is able to do work and to

1 Pfiügers Archiv, Vol. LXII (1895), p. 249.

2 Spallanzanr, Tracts of the Natural History of Animals and Vegetables.

3 Zeitschrift für physiologische Chemie, Vol. XII 
produce carbon dioxide without containing oxygen that can be exhausted by an air pump. ${ }^{1}$ Pflüger $^{2}$ and Aubert ${ }^{3}$ have shown the same to be true for the living frog. No one doubts that we are dealing with phenomena of splitting in these cases, which are at the same time the source of energy and the source for the motions, and the other physiological functions that go on in the vacuum. The differences observed by Spallanzani and Bunge in the length of time that animals live without oxygen may therefore be explained by assuming that different forms of animals contain different amounts of hydrolyzable substances. As soon as this material is used up "the clock stands still." Oxygen plays the rôle of replacing the substances capable of undergoing splitting which have been used up.

By calculating the energy obtainable by the hydrolysis and by the oxidation of carbohydrates Bunge has rendered it probable that in the higher animals the production of powerful work is not caused by hydrolysis alone, but by hydrolysis and oxidation. According to this, lack of oxygen could at once reduce the capacity for work of an animal by limiting it to the energy which can be obtained from hydrolysis.

Hoppe-Seyler was the first to suggest a chemical theory for the processes of oxidation which occur in the living organism.* He believes that, as in the process of putrefaction, reducing substances (such as hydrogen in the nascent state) are formed in all living cells through hydrolysis, and that these substances, when atmospheric oxygen is present, tear apart the oxygen molecule. The free oxygen atom is then in the condition in which it is able to bring about the oxida-

1 Untersuchungen über den Stoffwechsel der Muskeln (Berlin, 1867).

2 Pflügers Archiv, Vol. X.

3 Ibid., Vol. XXVI.

4 At the time I wrote this paper I was not familiar with the papers of Traube on the subject, which seem to give a more adequate presentation of the subject than Hoppe-seyler's hypothesis. [1903] 
tions which are characteristic of living matter. ${ }^{1}$ If, however, oxygen is absent, the chemical changes will be of an entirely different character. Compounds may be formed which are poisonous for the organism. Araki, for example, has shown that when oxygen is lacking considerable amounts of lactic acid and sugar appear in the urine. If the oxygen supply is normal, these compounds may also be formed from glycogen as intermediate products, but they are soon oxidized further. To the immediate consequences of lack of oxygen are therefore added those which are determined. through the presence of lactic acid in the body-for example, a diminution in the alkalinity of the blood. The cells of the kidney are also altered, as evidenced by the albuminuria which results when oxygen is lacking. ${ }^{2}$

While there exists a comparatively large number of investigations concerning the chemical side of the effects of lack of oxygen (of which we have mentioned only a few), we have very few biological observations on the same subject. Even careful search of the literature discloses little more than the fact that all animal life-phenomena cease sooner or later in the absence of oxygen, that in higher animals the phenomena of dyspnœa precede death, and that, as Kühne showed ${ }^{3}$ the protoplasm becomes vacuolated and opaque and disintegrates. The observations made upon mountain disease may also be mentioned under this heading. It did not seem possible to me that these facts exhausted all the biological effects of lack of oxygen. The fundamental importance of oxygen for all life-phenomena rendered it probable, a priori, that an accurate investigation would yield a series of qualitative and quantitative changes in life-phenomena. Such an investigation is, of course,

1 Physiologische Chemie, Vol. I, p.126.

2 Zeitschrift für physiologische Chemie, Vol. XIX.

3 Untersuchungen über das Protoplasma, 1864. 
rendered difficult by the fact that many animals soon die without oxygen, and I believe that this fact explains why this field has not thus far been the subject of more study. Still the duration of life is in most cases sufficiently long to demonstrate a series of such changes. Copepods are, for example, exceedingly sensitive to lack of oxygen; I know no other cold-blooded animals that die so rapidly without oxygen. Yet it is possible, as we shall see, to show definitely even in these animals that lack of oxygen affects their heliotropic sense in a most remarkable way; when deprived of oxygen negatively heliotropic Copepods become positively heliotropic.

Another consideration shows the importance of such investigation. Physiological chemistry alone may suffice to disclose the general sources of energy in animals. But the question as to how chemical energy is converted into the physiological activities of muscles, glands, etc., can of course not be answered by purely chemical researches. Molecular physiology must here bridge the chasm between the chemical changes and the outwardly manifested physiological activities of the organs. A complete understanding of the energetics of animals is not possible so long as we have no conception of the molecular changes which are brought about through processes of oxidation. It therefore seemed of importance to see whether such changes manifest themselves when oxygen is taken away. In this way arose the experiments detailed here on cleavage without oxygen, which I began three years ago, and which I discussed in a short note which appeared in Pflügers Archiv two years ago. ${ }^{1}$ I directed my attention to processes of segmentation because I considered these phenomena especially favorable for obtaining facts for a molecular physiology.

When we find that a physiological function is impossible

1 Pfügers Archiv, Vol. LV, p. 530. 
without oxygen, we are inclined to imagine that the given organ or organism lacks the necessary energy for performing this function. If we remember, however, that the conversion of chemical into the physiological function depends upon definite molecular conditions in the cells (state of matter, osmotic pressure, surface tension, phenomena of spreading, etc.), another explanation is possible, a priori: the cells are still able to produce the energy necessary for the physiological functions of the organ, but lack of oxygen led to molecular changes in the cells which prevented the conversion of the chemical energy into mechanical or other forms of energy. So far as I know, there are as yet no facts at hand to support such a view. Yet the following experiments, I believe, have led to positive results in this direction; for we can show that the eggs of Ctenolabrus and sea-urchin cannot segment without oxygen, and that, moreover, the already formed cleavage-cells of these eggs, especially those of Ctenolabrus, undergo certain structural changes when deprived of oxygen, which cause the cells to fuse together. It is possible that in this case we deal with a liquefaction of the membrane or the specific surface film of the cleavage-cells, and furthermore that the impossibility of the formation of a membrane in the absence of oxgen is why no cleavage occurs under these conditions. But no matter what one may assume regarding the formation of a membrane, it is clear that when separate cells fuse in the absence of oxygen, it is not to be expected that the unsegmented egg will be able to divide under these conditions. But those cells which have fused are by no means dead. When they are again supplied with air, segmentation sets in anew. We therefore see that the structural changes resulting from the absence of oxygen suffice to explain the failure of segmentation, and that it is not necessary in this case to attribute the latter to a failure of the source of chemical energy. This conclusion is further 
supported by the fact that the eggs of Fundulus in which such structural changes do not occur in the absence of oxygen even after twenty-four or more hours, continue to segment for more than twelve hours in the absence of oxygen.

What we are going to show for cleavage holds also for other life-phenomena, for example, the activity of the heart. We find that the heart of the Ctenolabrus embryo comes to a standstill so quickly after the oxgen has been withdrawn that it is impossible to think that the source of the energy for the beating of the heart has given out, while the heart of the Fundulus embryo continues to beat for many hours under similar conditions. It is possible that in this case also structural changes occur which are similar to those which we are able to observe directly in the cleavage-cells. These changes render impossible the transformation of chemical energy into mechanical energy in the contraction of the heart of the Ctenolabrus embryo.

Such molecular changes as manifest themselves by structural changes can be brought about just as well through processes of reduction due to the lack of oxygen as through the injurious compounds which may be formed in the absence of oxygen.

The facts which we obtained by the biological study of the effects of lack of oxygen may also again be of importance to the physiological chemist. We shall meet with some facts in this paper which will serve to illustrate the view of Bunge that in all probability the greater part of the energy necessary for considerable work of the muscles is furnished through processes of oxidation (and not through processes of hydrolysis). The frequency of the heart-beat of the Fundulus embryo decreases steadily during the period during which oxygen is withdrawn, until it reaches the minimum (of about twenty beats per minute), when all the oxygen has disappeared. But the heart can beat for ten hours at this rate 
at a temperature of $22^{\circ} \mathrm{C}$, in the entire absence of oxygen. This looks, indeed, as if the energy for a certain small number of beats could be furnished through hydrolysis alone, but that for a larger number of heart-beats (about 120) the energy is obtained, in the presence of oxygen, through oxidation.

\section{ON THE METHOD OF THE EXPERIMENTS}

In the following experiments on the effects of lack of oxygen, the oxygen was always displaced by hydrogen; the latter was freshly prepared from zinc and sulphuric acid for every experiment. The gas was washed thoroughly through two bottles of potassium hydroxide, one of potassium permanganate, and one of water. The hydrogen obtained in this way was entirely odorless. Before the experiment was begun, all air was driven out of the apparatus. The animals experimented upon were kept in an Engelmann chamber which permitted of direct microscopic observation.

In this method one fact is to be emphasized, which I have not seen mentioned elsewhere. When the living tissue upon which we wish to test the effects of lack of oxygen is placed in the Engelmann chamber, our judgment of the results is subject to the criticism that we do not know the exact moment at which the oxygen is all driven out of the living specimen. The resistances to the diffusion of oxygen out of the protoplasm are very great, and it may take considerable time before all the oxygen is removed. As long as we find that the phenomena whose dependence upon oxygen we wish to study cease immediately after the hydrogen is passed through the gas-chamber, this difficulty is less marked; for even though in this case not all of the oxygen had been driven out of the organism, it would show only that the particular function which is being studied already ceases at a diminution of the oxygen supply, and still more so in the total absence of oxygen. It is 
different, however, when the function which we are studying does not cease immediately. We are then unable to say whether the transitory persistence of the function shows that not all of the oxygen has been driven out, or that the given function is not directly dependent upon oxygen. Things of this sort confront us when we attempt to decide whether cleavage is possible without oxygen. We find that when sea-urchin eggs are placed in an Engelmann chamber immediately after fertilization, and we begin to pass hydrogen through it, the eggs not only cleave into two, but often even into four, cells; but this cleavage occurs within the first fifty or eighty minutes after fertilization, and it might be thought that it takes a longer time than this to drive out all the oxygen. To be certain on this point in such cases, I made use of the following procedure: I introduced the eggs in which I wished to study the dependence of cleavage upon oxygen into an Engelmann chamber which was kept on ice. Hydrogen was then passed through the apparatus. The low temperature inhibits cleavage. In order to discover when I could take the eggs off the ice, and know that the objection could no longer be raised that the eggs still contain oxygen, I introduced a second gas-chamber into the circuit. This contained eggs of the same culture, and through it I passed the same current of gas as that which went through the first. The second, control, chamber was not put on ice. As long as a trace of cleavage continued in this control chamber, there was reason to suspect that not all the oxygen was driven out. As soon, however, as cleavage ceased, it seemed reasonable to assume that, even though not all the oxygen had been driven out of this chamber, the portion which remained behind was no longer sufficient to start cleavage. It must be remembered, however, that the eggs kept on ice had not lost as much oxygen as the control eggs during this time, for oxidation did not occur as rapidly in the 
former as in the control eggs. It was therefore necessary, after cleavage had ceased in the control eggs, to pour the current of hydrogen through the Engelmann chamber for some time before the eggs to be experimented upon were removed from the ice, and the real experiment was begun.

The objection might be raised that the prevention of cleavage through the ice injured the eggs. I guarded against this objection by the following control experiments: First of all the eggs were again exposed to the air after the completion of the experiment, and their cleavage observed. If this took place in a normal way, its absence in the lack of oxygen could not have been the effect of the cooling. Secondly, another portion of the eggs of the same culture were put upon the ice at the same time and for the same length of time as the eggs used in the experiment, only they remained exposed to the air. I will state at once that these eggs always segmented when brought back to room temperature. During the entire time of the experiment hydrogen passed uninterruptedly through the Engelmann chamber, not only to guard against possible leaks in the apparatus, but also to remove the carbon dioxide formed. The latter is absolutely necessary.

III. RESEGMENTATION OF THE CTENOLABRUS EGG WITHOUT OXYGEN

The older experiments of Spallanzani, Dutrochet, Saussure, and Schwann had already established the fact that in permanent lack of oxygen the development of plants and of animal eggs is impossible. Paul Bert added to these observations the fact that when the air contains only 3.4 per cent. of oxygen certain plants cease to germinate. In these experiments, however, the question as to whether celldivision is at all possible without oxygen was not touched upon. Three years ago I began experiments on the eggs of 
Fundulus which showed that the egg not only segments when oxygen is removed by pyrogallol, but even continues to develop for about sixteen hours. Demoor, who was unfamiliar with my experiments, began experiments on Tradescantia cells in which he found that a cell-division which had already started at the time that the oxygen was being removed continues to the completion of nuclear division, but that the subsequent cell-division does not occur. $\mathrm{He}$ concludes from this, first, that cell-division is impossible without oxygen, and especially that without oxygen the cellmembrane cannot be formed; and, secondly, that the nucleus may divide without oxygen, that it is anaërobic. ${ }^{1} \quad$ I have in a previous paper pointed out the incorrectness of the second conclusion.

My own experiments, which I will give here, were made on fish eggs (Ctenolabrus and Fundulus) and sea-urchin eggs.

The egg of Ctenolabrus, a marine Teleost, is perfectly transparent and free from pigment, and the changes which are described in the following pages can be studied with great accuracy under the microscope. The eggs which were used in the following experiments were always fertilized artificially in the laboratory.

If the freshly fertilized eggs of Ctenolabrus are introduced into an Engelmann chamber, and care is taken that all the air is driven out of the apparatus before the experiment is begun, and the stream of gas is maintained, the eggs cleave, without exception, into two cells, and in most cases even into four cells. Occasionally they even go into the eight-cell stage. If the eggs are introduced into the gaschamber not immediately after fertilization, but in one of the later stages of cleavage, two or three divisions of all the cells still occur.

1 Archive de biologie, Vol. XIII. 
Must we now assume that Ctenolabrus is able to divide two or three times without oxygen? The first cleavage of the Ctenolabrus egg occurs in from fifty to seventy minutes after fertilization, according to temperature; the second, about fifteen to thirty minutes later. It is entirely possible that even in a strong current of hydrogen all of the oxygen is not driven out of the eggs in so short a time. In order to settle this point, I made a long series of experiments in the manner described above, in that I introduced the eggs immediately after fertilization into two gas chambers, one of which was kept on ice, while the other was exposed to room temperature, and passed the same stream of hydrogen through both. I will describe a few of these experiments here. In order to be brief, I will call the eggs upon the ice the experimental eggs, the others the control eggs.

In one experiment the control eggs divided into two cells fifty minutes after fertilization, when the experimental eggs were removed from the ice, while the stream of hydrogen was kept up uninterruptedly. In thirty minutes the first cleavage occurred in the experimental eggs. At the same time the control eggs went into the four-cell stage, and twenty-five minutes later the experimental eggs also went into the four-cell stage. Cleavage then ceased in both chambers. Even though hydrogen had been conducted through the chamber containing the experimental eggs, which had been kept on the ice for a long time before the beginning of cleavage, and the oxygen had probably been driven out more thoroughly than in the control eggs, cleavage nevertheless occurred in the same way in both. There was only one difference; all the control eggs reached the four-cell stage, while about 25 per cent. of the experimental eggs remained in the two-cell stage.

In another experiment the experimental eggs remained for one hour and forty minutes on the ice. During the first 
hour all of the control eggs had developed into the two-cell stage, but did not go any farther. When exposed to room temperature, an incomplete division occurred in a small number of the experimental eggs after thirty minutes. Only a suggestion of a membrane dividing the two cells was formed; the peripheral cell-membranes were not formed. The process then came to a standstill. That this result was attributable to the lack of oxygen, and not to the prolonged stay in the cold, was shown by the fact that when, after some time, the gas-chamber was opened, vigorous cleavage set in in all the eggs after thirty minutes.

The experimental eggs in a third experiment remained on the ice for two hours. The control eggs reached the fourcell stage within the first eighty minutes. Cleavage then ceased. When the experimental eggs were taken from the ice, not a suggestion of cleavage set in during the following eighty minutes. Air was then admitted. All the eggs began to divide in thirty minutes.

I obtained the same result in more than ten further experiments. With the exception of the fact that in an occasional egg among hundreds an intimation of a dividing membrane was visible, no cleavage whatsoever occurred when a vigorous stream of hydrogen was led for two hours or longer before the beginning of the experiment through the gas-chamber which contained the experimental eggs and was kept on the ice. Yet the same eggs all divided within half an hour when later exposed to the air.

It might be thought that lack of oxygen only markedly retards cleavage, but does not bring it to a complete standstill. Yet it did not matter how long one waited-cleavage never occurred in the gas-chamber in the case of lack of oxygen, when all the oxygen had been driven out.

Furthermore, I ascertained that when any segmentation whatsoever occurred in a weak stream of hydrogen, it always 
occurred at the same time as (or earlier than) in the eggs from the same culture kept in oxygen. If the minimum amount of oxygen necessary for cleavage is present, the velocity of the cleavage is a function of the temperature and not of the amount of oxygen present. It is important to emphasize the fact that lack of oxygen at room temperature does not retard cleavage, as does a reduction in the temperature.

Finally, I convinced myself of the fact, through a special series of experiments, that prolonged exposure to cold does not diminish the power of the egg to divide. I allowed a weak current of hydrogen to pass through a gas-chamber which remained on ice for four hours. When I then exposed the eggs to room temperature and continued to pass the same weak current of gas through the chamber, all the eggs divided. The majority reached the four-cell stage, and a few even the eight-cell stage. Cleavage then ceased. If not all the oxygen is driven out, cleavage proportionate to the amount of oxygen present still occurs in spite of the prolonged cooling. We are, therefore, justified in concluding that when all the oxygen which it is possible to remove from the Ctenolabrus egg is driven out, no complete celldivision can occur.

The question now arises in how far a division of the nucleus is possible in such an egg. At the surface of the Ctenolabrus egg a series of visible changes occurs before the first cleavage. In the center of the nucleus several droplets of a strongly refractive substance collect, which increase in number and size, then coalesce, to resolve again into a large number of minute droplets just before cell-division. These droplets probably play a rôle, as we shall see later, in the process of cell-division. It is possible, but not proved, that their formation is a function of karyokinesis. These changes in the strongly refractive substance also occur 
when all the exhaustible oxygen has been removed so that cell-division is no longer possible. The energy necessary for these changes must probably therefore be obtained from processes of hydrolysis.

One might be tempted to believe that the nucleus could continue to divide without oxygen, while the cell remains undivided - a phenomenon I discovered in sea-urchin eggs, when they are brought into sea-water of a certain concentration. In such eggs the number of nuclei steadily increases, but no cell-division occurs. But such phenomena certainly do not take place in the absence of oxygen. Eggs were freed from oxygen by passing hydrogen over them for two hours while on ice. They were then exposed for one hour to room temperature, while the flow of hydrogen was not interrupted. No cleavage had occurred. The eggs were then killed and sectioned. It was impossible to find more than one nucleus in these eggs; this was, however, in a number of instances undergoing mitosis. The experiment was repeated with the same result. One mitotic division may therefore occur without oxygen, but no more.

If eggs which have been freed from oxygen for a sufficiently long time while on ice, and which have shown no evidence of cell-division when exposed to hydrogen for an hour at room temperature, are exposed to the air, they all divide in the course of thirty to fifty minutes. But they do not then first divide into two cells and later into four, but immediately into four - occasionally into three or five cells. This also occurs when a strong stream of hydrogen is sent through the gas-chamber for three and a half hours at a low temperature before the experiment is begun - under conditions therefore when, in all probability, all of the free oxygen has been removed from the eggs. Two divisions of the nucleus therefore always occur-one in the hydrogen, and one after the admission of air-before the first cell-division 
is inaugurated. This marked retardation of cell-division has its basis in peculiar molecular changes, which we will discuss in detail in the following sections of this paper.

\section{THE FUSION OF CLEAVAGE-CELLS THROUGH LACK OF OXYGEN}

The fact that the egg of Ctenolabrus is not able to segment without oxygen may be due to one of two causes: first, processes of oxidation might be the only source of energy for segmentation; second, it might be possible that, even though enough chemical energy for segmentation can be obtained from hydrolysis, yet this chemical energy cannot be connected with the chemical energy necessary for cleavage because of the structural changes brought about by the lack of oxygen. Demoor concludes from his experiments on Tradescantia that no cell-wall is formed without oxygen, and that in consequence no cell-division occurs without oxygen. Demoor brings no positive proofs for his view. In the case of the Ctenolabrus egg, however, we can show that structural changes occur in cleavage-cells, in consequence of which these cells fuse together. It is conceivable that the same structural changes must also hinder the segmentation of the freshly fertilized egg. The sketches, Figs. 103-8 were made with the camera lucida and were all taken from the same egg. The egg was fertilized at $10 \mathrm{~A}$. M., and immediately thereafter introduced into a gas-chamber and kept in a current of hydrogen. Cleavage took place in the normal way, and since the current of hydrogen was not very strong, even the eight-cell stage was reached (Figs. 103-5). A series of degenerative changes then set in. At first a gathering of the strongly refractive droplets, which we have described already, was formed in the two main furrows (Figs. 104 and 105) and some furrows began to become indistinct. Fifteen minutes later the greater portion 
of the peripheral cell-limits had already become invisible (Fig. 106), and after another fifteen minutes nothing could be recognized of the entire blastoderm except a collection of droplets which had fused into larger drops (Fig. 107). In the next two hours the latter only became more spherical, but otherwise under-

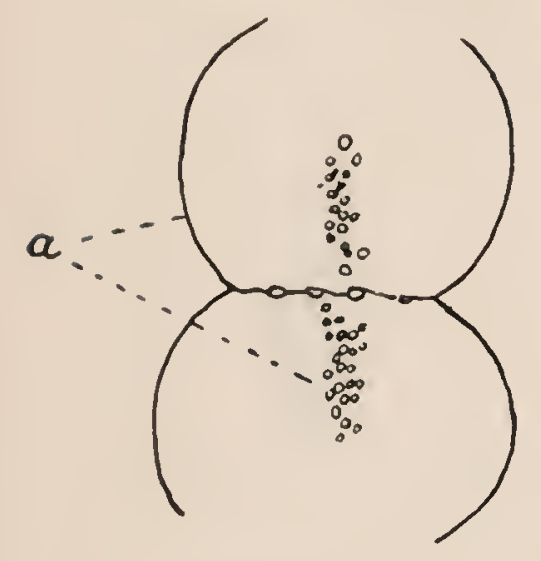

FIG. 103 went no change (Fig. $\boldsymbol{f}$. 108). The germdisk was optically still less visible than in the unfertilized egg. It therefore required only thirty-five minutes after cleavage came to a stop, for

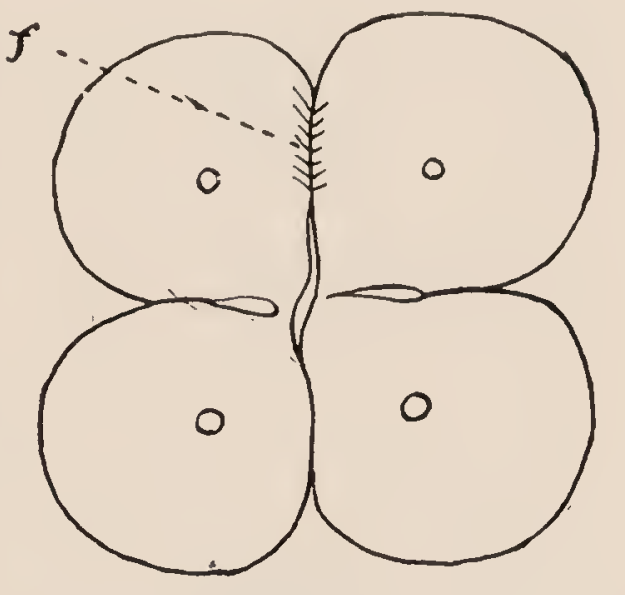

FIG. 104

the complete liquefaction of the cleavage-cells of an eightcelled blastoderm. It is scarcely necessary to mention that the same process in various experiments took a little more or a little less time.

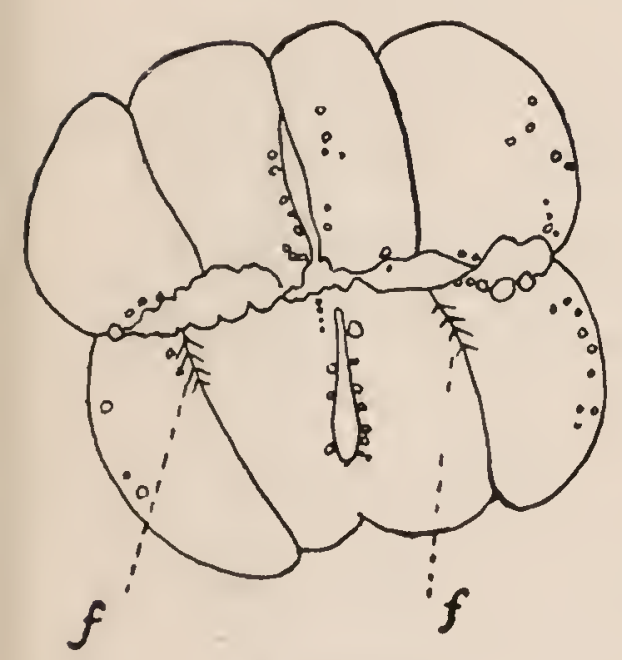

FIG. 105

What we observe here is found in every such experiment upon Ctenolabrus eggs, the only difference being in the form and the arrangement of the droplets of the strongly refractive material, which at times may form a more perfect cast of

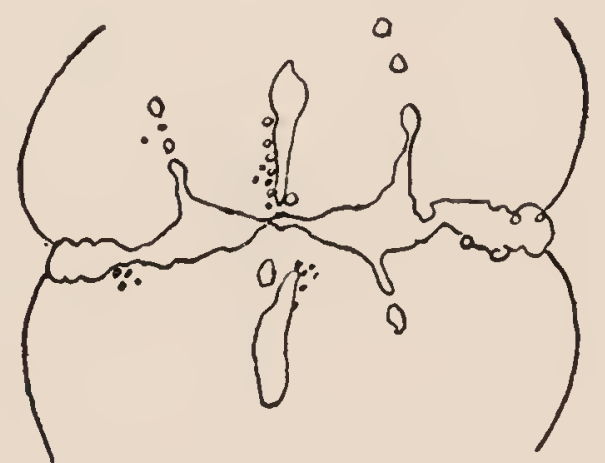

FIG. 106 the old lines of cleavage than in the experiment described. Even when the oxygen is driven out so slowly that the egg has time to reach the sixteen- or the thirty-two-cell stage in the stream of hydrogen, the same series of degenerative changes occurs as soon as cleavage has come to a standstill. 
The question now arises whether this liquefaction or fusion of the cleavage-cells occurs equally well and with the same rapidity in every stage of development. As soon as the eggs have reached the sixty-four-or the one-hundred-and-twentyeight-cell stage, the behavior toward lack of oxygen is some-

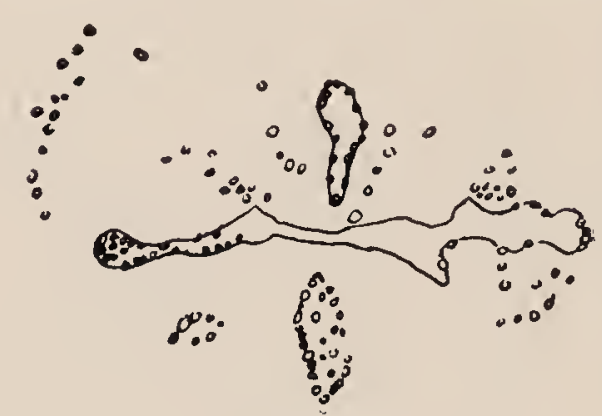

FIG. 107

what different. While in an egg which is in the eight-cell stage the cells fuse in about one hour in the absence of oxygen, a liquefaction of the cleavage-cells also occurs in the eggs in the sixty-four- and onehundred-and-twenty-eight-cell stage,

but only at the periphery of the blastoderm, and even here more slowly than in the cells in the earlier stages of segmentation. The droplets of the refractive substance appear in the furrows, but they smaller than in the eggs in an earlier stage of development, and it is for this reason perhaps that large oil drops are

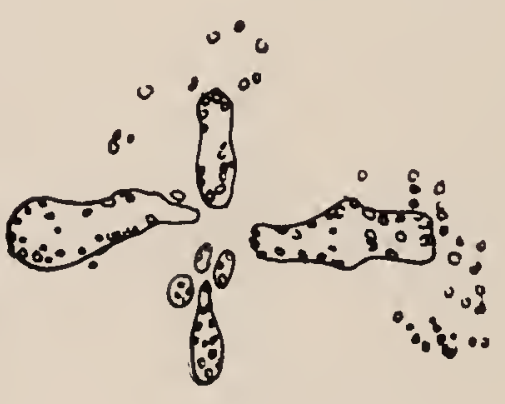

FIG. 108 formed less easily. Figs. 109-111 illustrate the process of

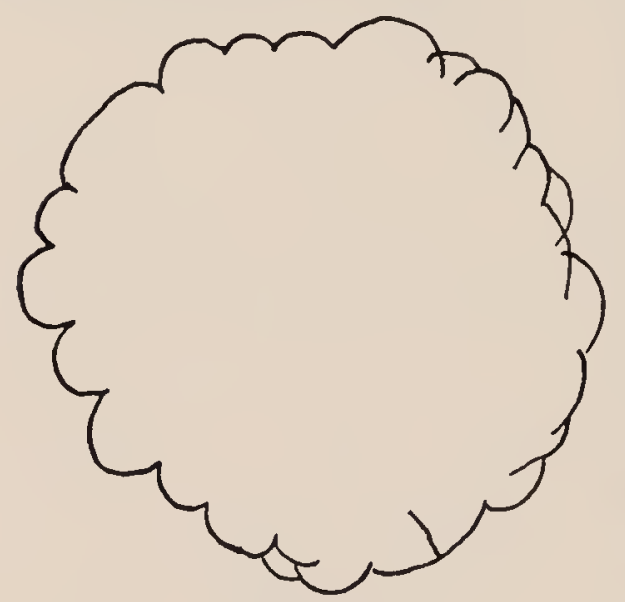

FIG. 109 liquefaction in such an egg. The egg was put into the gas-chamber at 2:25 o'clock while in the sixty-four-cell stage. At this time its shape was sketched with the camera lucida (Fig. 109). The outlines of the cells within the blastoderm are not shown. The segmentation at first continued. At 4 o'clock liquefaction was very distinct at the periphery. It occurred in this way that in individual cells at the periphery of the blastoderm the outline at first becomes invisible, after which the entire cell gradually disappears. Through this disappearance of the 
cells at the periphery the blastoderm becomes smaller (Fig. 110). At 6:35 o'clock the liquefaction of the cells at the periphery had progressed much farther (Fig. 111). The diameter of the blastoderm was only a little more than threefifths of the diameter the egg possessed four hours earlier

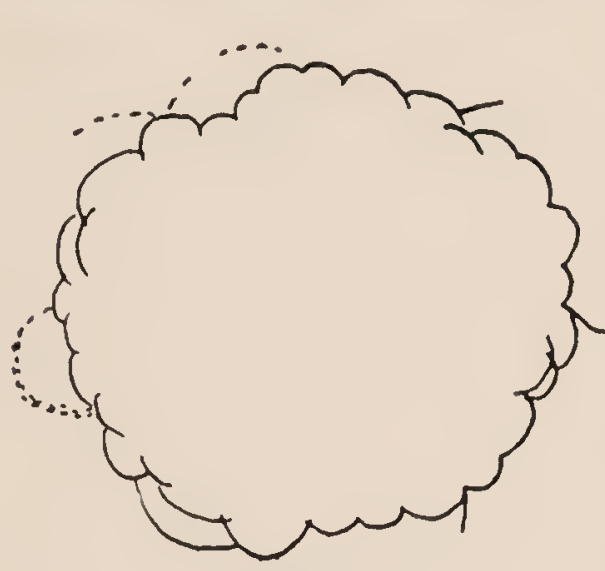

FIG. 110 when it was in the sixty-four-cell stage. Around the blastoderm lay granular masses, which were in all probability the remains of the liquefied cells. Soon thereafter a change (shrinking?) occurred in the yolk, which served to close the experiment. The disappearance of the cleavagecells occurs more slowly therefore in the later stages of development than in the earlier stages of developmént.

It may be of interest to raise the question: In what do these peculiar structural changes consist which lead to the fusion of the cleavage-cells in the absence of oxygen? If we wish to answer this question, we must acquaint ourselves more fully with the history and the significance of those peculiar refractive substances which appear in droplets. Soon after fertilization, before the union of the pro-nuclei, one observes in the center and upon the surface of the germ the appearance of several strongly refractive droplets. These undergo, as has already been said, a series of changes, of which the most remarkable is this, that shortly before the first cleavage a single system

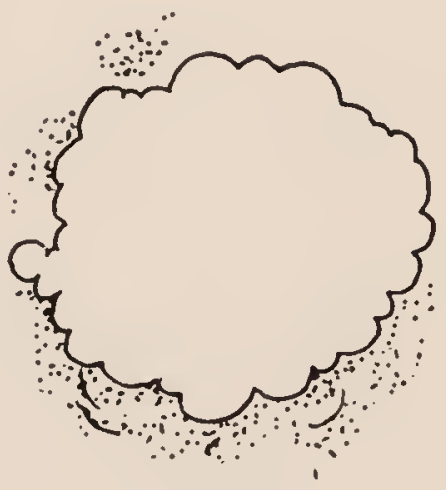

FIG. 111 of radiations coming from a common center is formed, which looks very much like the radiations about a centrosome. These radiations might be a process of emulsion, for the radii break up very rapidly into small droplets which are strongly 
refractive, and soon thereafter the center also breaks up into such droplets. As soon as the blastoderm divides, these droplets are found distributed over the entire surface, collected especially thickly along the furrow between the two cells. Before the next cleavage occurs, these droplets again arrange themselves in a line which corresponds to the next furrow (Fig. $103, a)$. In the process of segmentation part of these droplets disappear. This fact, in conjunction with a series of other facts, with which we shall become acquainted in the next section of this paper, leads me to suspect that this strongly refractive substance serves for the formation of the membrane of the cleavage-cells of the Ctenolabrus egg. ${ }^{1}$ That such a membrane, or at least a solid surface layer, covers the cleavage-cells of the Ctenolabrus egg immediately after a cleavage is completed I have observed directly; for folds are often formed on the surface of the cells, which are especially distinct immediately after a cell-division in the furrow (Figs. 104 and 105, f). On the assumption of the existence of a membrane our observations can be expressed in a simple way. In the absence of oxygen the membranes of the cleavage-cells are liquefied and this brings about the fusion of the latter. The material of which the cell-walls were formed flows together in droplets which coalesce into larger drops in the center of the germdisk. This liquefaction of the material of which the membrane is formed also renders cell-division impossible in the case of lack of oxygen. The assumption of the existence of a membrane, or at least of a specific surface film, in animal cleavage-cells also brings the mechanics of cell-division in animals and in plants into better harmony.

The fact that in the process of cleavage the droplets always collect along the plane in which cleavage is to occur later is, as I would suggest in passing, a corroboration of

1 It is now generally assumed that the surface film of cells is formed by lipoids. The optical appearance of the droplets mentioned in the text is indeed that of a fatty substancs. [1903] 
the view I expressed a short time ago on the mechanics of cell-division. ${ }^{1}$ I imagine that as soon as the nucleus divides, vortex motions take place about each of the two daughternuclei, which leads to a tearing apart of the cell-contents; in other words, to cell-division. ${ }^{2}$ If this assumption is correct, movable particles must collect where the two vortex motions meet - that is, along the lines in which cleavage is to occur later. We indeed find this to be the case in the Ctenolabrus egg, and also in such eggs as carry pigment at their surfaces.

These vortex motions carry the droplets to the place where the next cleavage is to occur, and where they are necessary for the formation of a membrane - a remarkable example of that "purposeful" interaction between mechanical conditions which we meet so often in processes of development.

We see, therefore, that molecular changes--apparently a liquefaction and an emulsion of the membrane or the surface film of the cleavage-cells - occur in the case of lack of oxygen which gives an adequate explanation of the fact that no cleavage occurs in Ctenolabrus eggs without oxygen. But the fact that nuclear division also soon comes to a standstill indicates that changes corresponding to those in the membrane must also occur inside the cells.

\section{REVERSAL OF THE EFFECT OF LACK OF OXYGEN UPON ADMISSION OF AIR}

When an egg whose entire blastoderm has become invisible in hydrogen is again exposed to air, the changes which ensue differ according to the length of time during which the eggs have been exposed to the current of hydrogen. If the egg remains too long without oxygen at room tempera-

1 Archiv für Entwicklungsmechanik, Vol. I.

2 I find in this case, as in that of all hypotheses, that they do not gain in attract. iveness with growing age. Conklin, though, has accepted the hypothesis of vortex motions and Butschli has justly claimed priority for it. [1903] 
ture, it dies. If the experiment is interrupted early - that is, when the cell-walls have just begun to become indistinct - all, or at least a part, of the cell-membranes again become visible upon admission of air. Under these circumstances,

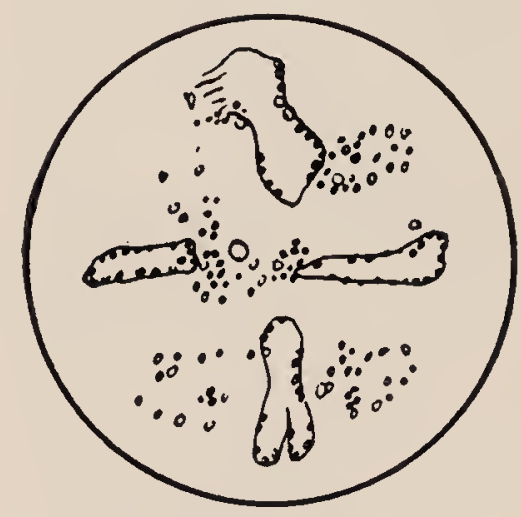

FIG. 112 however, every cell usually divides, not into two, but into four cells which corresponds with what has been said before.

When we wait a little longer before admitting air, a circular blastoderm is at first formed in which no trace of cleavage is visible. The blastoderm then suddenly breaks up into a large number of cells at once, but curiously enough this cleavage is confined, in most cases, to the periphery of the blastoderm. In this case also the refractive substance which has been described plays a peculiar rôle. Figs. 112-

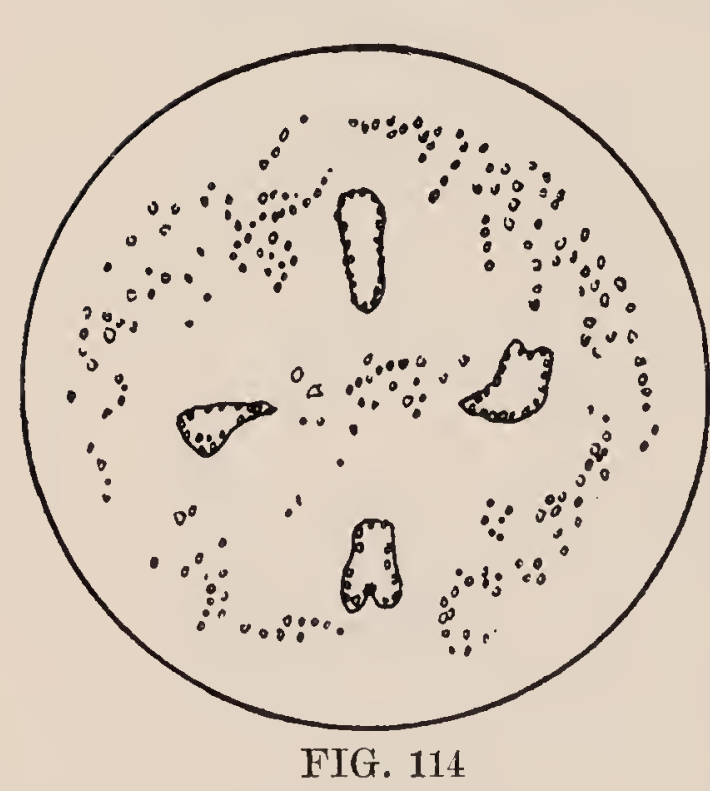

17 represent the various

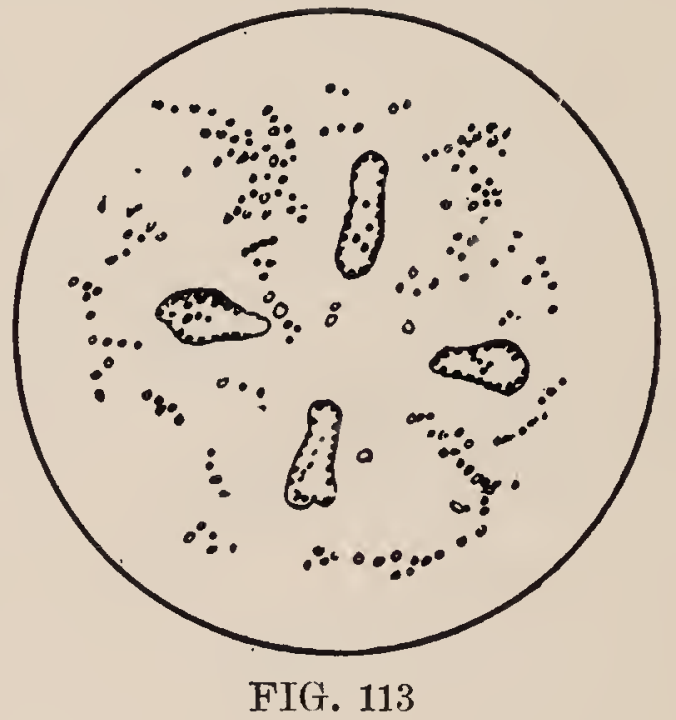
stages of the renewed cleavage of the same blastoderm in which we studied the disappearance of the lines of cleavage in hydrogen (Figs. 103-8). Fig. 108 shows the condition of the blastoderm in hydrogen at 2:10 o'clock. Only four large drops of the refractive substance, surrounded by droplets of smaller size, permit one to recognize the place of the blastoderm. At 2:18 pure oxygen was sent through the gas-chamber. At first the smaller droplets separated from the surface of the large droplets and moved toward what had been the periphery of the blastoderm. (Previously, 
during the liquefaction of the cell-walls, they had moved toward the center of the blastoderm.)

At 2:35 a unicellular spherical blastoderm became visible (Fig. 112). The larger central drops gradually broke up

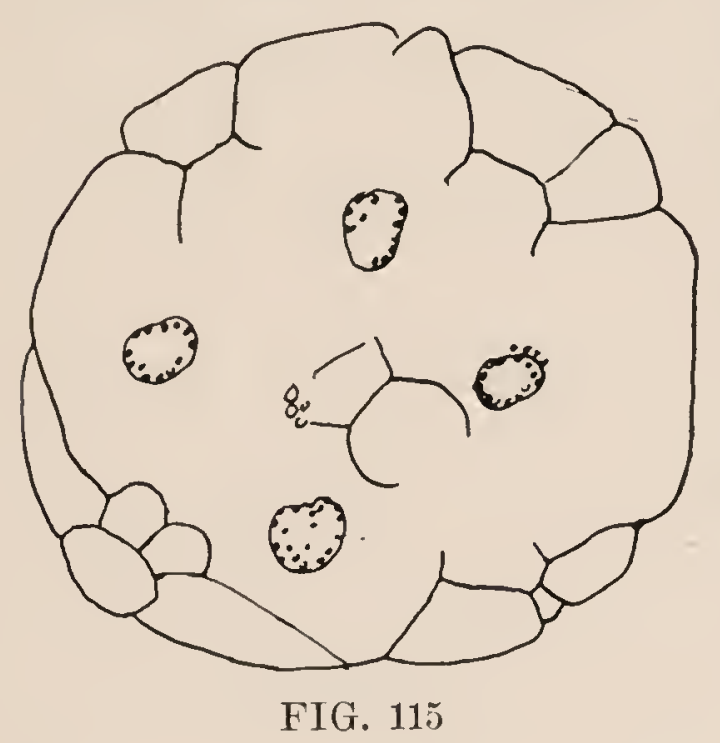

tiny droplets have collected, namely, at the periphery. The periphery broke up into about eighteen cells at once (Fig. 115). These cells about corre-
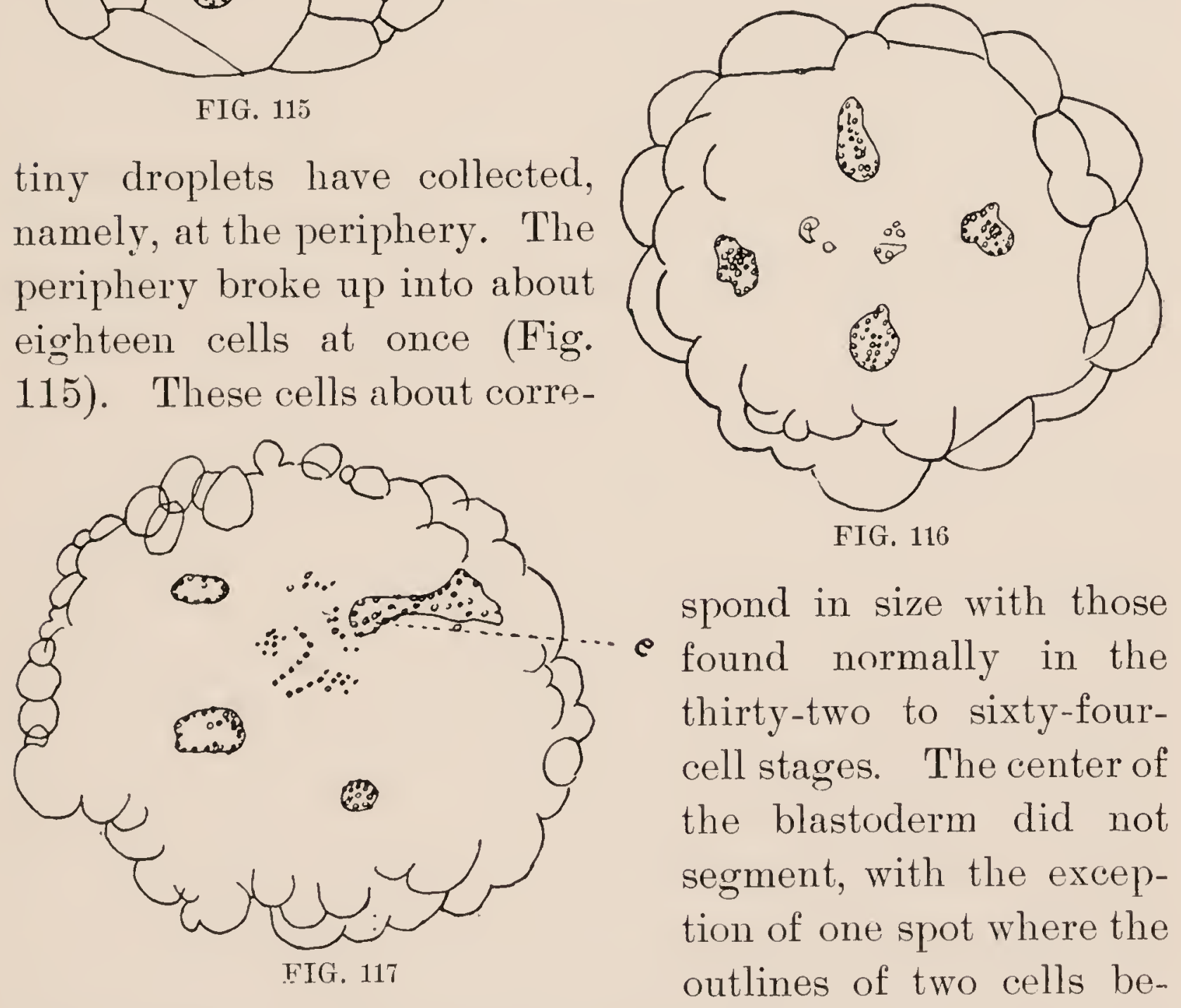
came visible. I had noticed previously a small collection of the refractive droplets at this point.

Still another relation between the distribution of the droplets and segmentation is noticeable. If the reader will 
compare Figs. 114 and 115 he will notice that more cleavagecells are formed in the four sectors which lie between the four large drops, and which contain the larger number of small droplets at the periphery, than in the four sectors which contain the large drops and a smaller number of small droplets. This relation may be accidental, but I may be allowed to state that when Fig. 114 was formed I expected from my earlier observations precisely that type of cleavage which is shown in Fig. 115.

The cells formed at the periphery continued to divide, while the center remained undivided. The two cells which had first been perceived there disappeared again. The four central drops became steadily smaller, and one of them broke up entirely into small droplets, as if a slow emulsion took place (Fig. 117,e). In this way the blastoderm changed, within fifty minutes, from the condition shown in Fig. 115 into that shown in Figs. 116 and 117. Development then ceased. The long exposure to lack of oxygen at a relatively high temperature led to an early death of the germ.

The phenomena shown in Figs. 112-17 are typical. The exceptions which one encounters are connected with differences in the behavior of the small droplets of the strongly refractive substance. In this connection I must mention the fact that a small percentage of the eggs also showed segmentation in the middle of the blastoderm. In these cases, however, I usually (if not always) found that not only the periphery, but the entire blastoderm, was studded with very minute droplets immediately before cleavage. The large central drops were then found to dissolve rapidly (emulsion?). I also found in rare cases, though, that only one sector of the blastoderm divided, while the rest remained undivided. In these cases also the small refractive droplets were usually collected in this sector. These facts all support the idea that the refractive substance forms the membranes of the cleavage spheres. 
VI. THE EEFECT OF CARBON DIOXIDE UPON THE PROCESSES OF CLEAVAGE IN THE CTENOLABRUS EGG

If eggs are introduced into a current of pure carbon dioxide (which has been carefully washed), we must expect to obtain, besides the effects of mere lack of oxygen, the specific chemical effects of the $\mathrm{CO}_{2}$. Even though everything indicates that the action of $\mathrm{CO}_{2}$ is qualitatively different from the action of simple lack of oxygen, such differences have only rarely to my knowledge, been demonstrated directly in the cell. ${ }^{1} \quad$ In the egg of Ctenolabrus, however, these differences are very striking. If freshly fertilized eggs are introduced into a stream of pure $\mathrm{CO}_{2}$, no trace of cleavage occurs, even though the eggs are not kept on ice. Under similar external conditions the eggs kept in hydrogen divided two or even three times. The germs also die much more rapidly in $\mathrm{CO}_{2}$ than in hydrogen. This constitutes, however, only a quantitative difference. A qualitative difference evidences itself, however, immediately that the air is replaced by a current of $\mathrm{CO}_{2}$ in eggs in the two- or four-cell stage. In these experiments the eggs were kept in a drop of sea-water in an Engelmann gas-chamber. Amoboid movements (which were first noticed at the periphery of the drop) took place on the surface of the egys in some ten to fifteen minutes, when a current of carbon dioxide was passed through the chamber. Whether the whole protoplasm or only the superficial layer of the protoplasm takes part in these changes could not be determined. I have made a series of camera drawings of these movements, which I will reproduce here.

Fig. 118 shows the outlines of the four cells of an egg at the beginning of the experiment. Fourteen minutes later this cell had the appearance shown in Fig. 119. One of the four cells, that which was directed toward the periphery of the drop and first struck by the stream of carbon dioxide, sent out amœ-

1 See Loeb and Hardesty, Pflügers Archiv, Vol. LxI. 
boid pseudopodia. A few minutes later all of the cells sent out such pseudopodia, which soon became shorter, however, as if the substance of the pseudopodia had been torn, e.g., through an emulsion (Fig. 120). The outlines of the germ

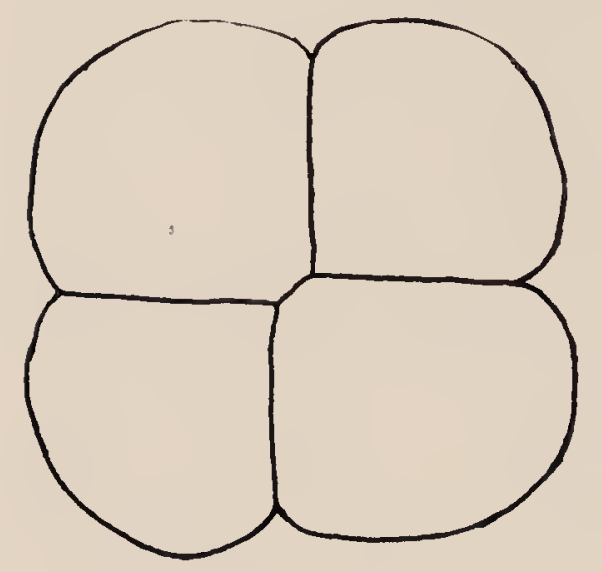

FIG. 118 then again became smooth, but not entirely so (Fig. 121), and finally the blastoderm gradually disappeared (Fig. 122). The entire series of changes shown in Figs. 118-22 took about fortyfive minutes. Besides these changes, another series took place in the blastoderm and the yolk, which, however, I am not as yet able to interpret, and which I therefore do not describe, as their description would take up much room without at present being of

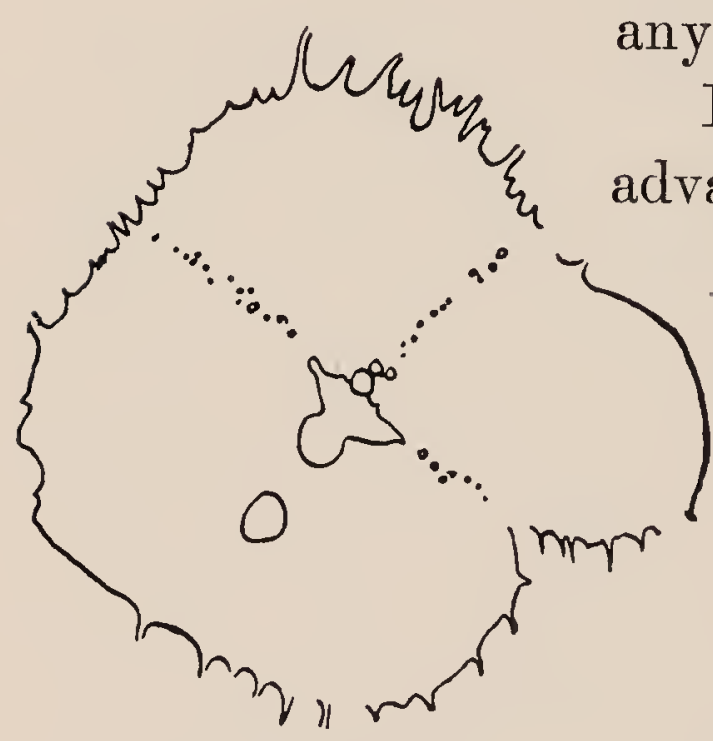

If eggs in an advanced state

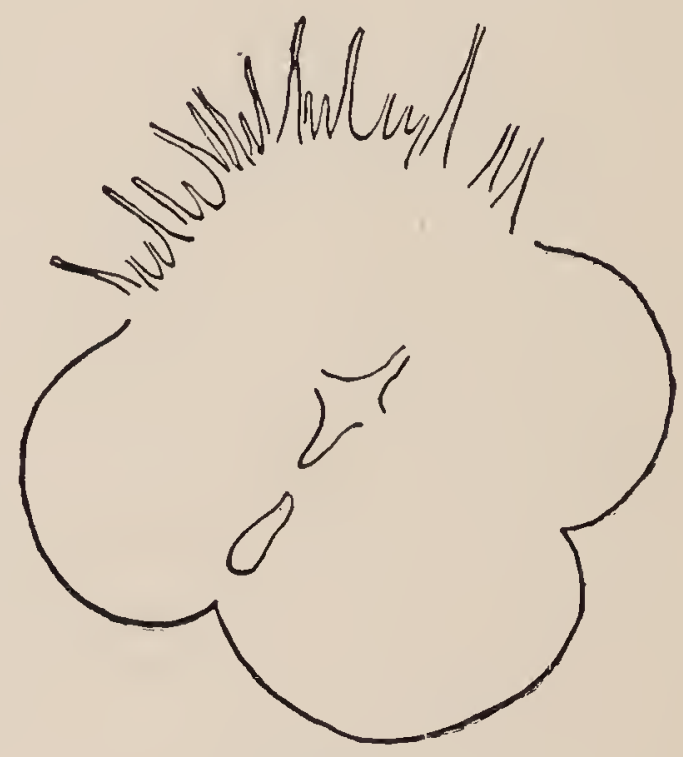

FIG. 119 of division are introduced into $\mathrm{CO}_{2}$, a solution of the cleavage-cells occurs at the periphery just as in hydrogen. VII. THE EFFECT OF PURE OXYGEN UPON CLEAVAGE

In embryological literature one FIG. 120 at times encounters the statement that the processes of development in pure oxygen at atmospheric pressure go on differently from those in air. Demoor also states that nuclear division is accelerated in pure oxygen.

Now, it is one of the established facts of physiology that 
the consumption of oxygen is, within wide limits, independent of the partial pressure of the oxygen, and that it makes normally little difference for the processes of oxidation whether we breathe air or pure oxygen. Still, in order to determine

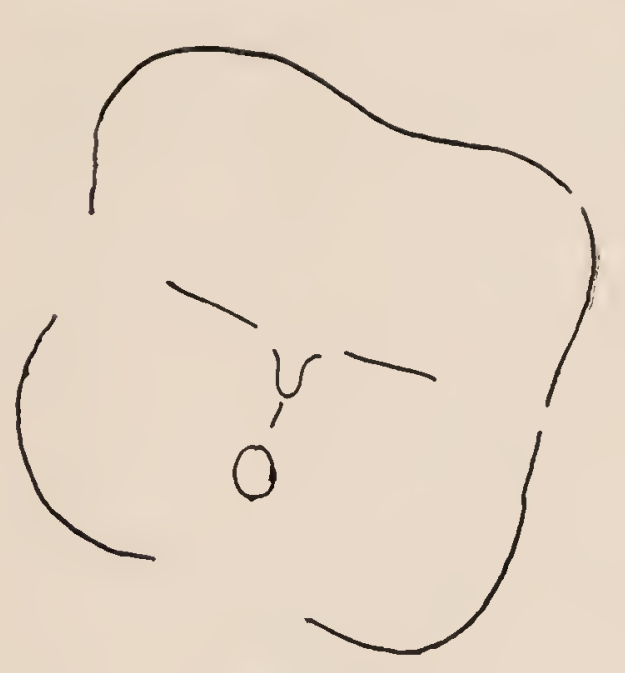

FIG. 121 experimentally the action of pure oxygen upon cleavage, I made the following experiments.

An inverted ten-liter bottle $A$ (Fig. 123) was filled with pure oxygen. A long glass tube $a$ and a short one $b$ passed through the rubber stopper in the bottle. The glass tube $a$ was connected with an Engelmann gaschamber $I$. The short glass tube $b$ was connected with a longer tube $c$, and the bottle $B$ was filled at the beginning of the experiment with water. A second short glass tube passed through the stopper of the latter and was connected to the Engelmann gas-chamber $I I$. The connecting rubber tube between $A$ and $B$ was filled at the beginning of the experiment with water and closed by a pinch-cock. As, soon as the pinch-cock was opened the oxygen was driven out of $A$ through the gaschamber $I$ by the flow of the water out of $B$, and the same amount of air was suctioned through the gaschamber II into t, - bottle $B$. In this way the effect of pure oxygen could be compared with that of atmospheric air. A few important but self-

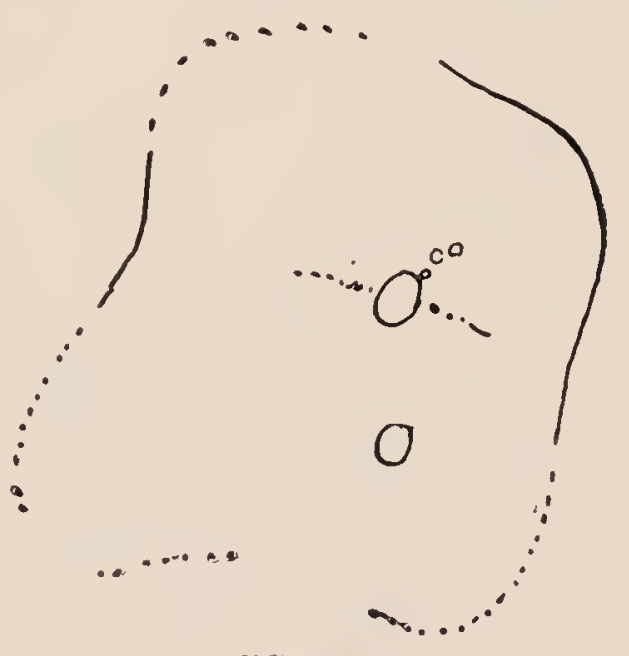

FIG. 12: evident details in the arrangement of the experiment have been omitted in the drawing.

In one experiment eggs which had been in the eight-cell stage, but the cleavage-cells of which had been fused by ex- 
posure to a current of hydrogen, were introduced into both gas-chambers. I wished to determine whether the renewal of segmentation would occur more rapidly and differently in pure oxygen than in air. The result was that after fifty minutes cleavage occurred almost simultaneously in both gas-chambers and in exactly the same way. Cleavage occurred only at the periphery and the cells which were formed were about the size of those found in the thirtytwo- or sixty-four-cell stage. In a second experiment cleavage occurred even a little more rapidly in the air than in pure oxygen. For the rest things were about the same. Under these circumstances I saw no reason for continuing these experiments; they showed clearly enough that it does not matter, so far as the renewal of cleavage of liquefied Ctenolabrus eggs is concerned, whether air or pure oxygen is
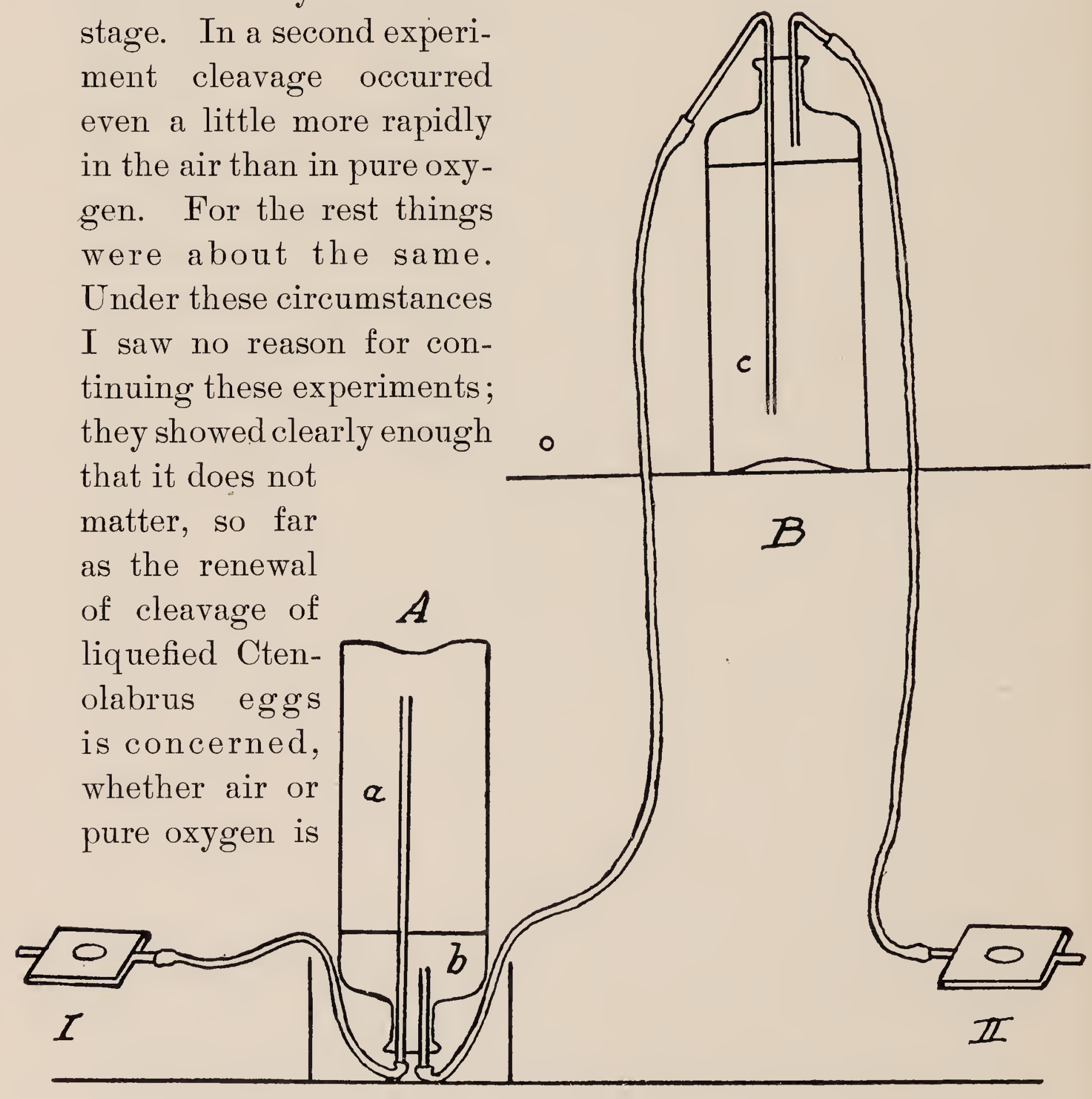

FIG. 123 
supplied to them. We found before that a lack of oxygen does not retard cleavage as long as cleavage is at all possible. In the same way an excess of oxygen does not accelerate the process.

VIIT. EFFECT OF LACK OF OXYGEN ON THE CLEAVAGE OF THE FUNDULUS EGG

The eggs of Ctenolabrus have a lower specific gravity than sea-water, and therefore float at the surface of the water. Here they find the oxygen necessary for their development. If the eggs of Ctenolabrus with their great need for oxygen had a specific gravity large enough to cause them to sink to the bottom, they could scarcely develop in many places, since at the bottom of the ocean where processes of putrefaction are going on, the tension of oxygen is much less than at the surface. We may therefore expect, in general, that fish eggs which sink to the bottom of the ocean and develop there are much more independent of oxygen than the egg of Ctenolabrus. This is really often the case. The egg of Fundulus has a greater specific gravity than seawater and develops at the bottom of the ocean. I have shown that the egg of Fundulus can develop for some time in the absence of oxygen. In these experiments the eggs were introduced with a few drops of sea-water into a small glass tube sealed at its lower end, and this tube was put into a test-tube containing several cubic centimeters of an alkaline pyrogallol solution. The test-tube then was sealed at the top. The pyrogallol solution was prepared according to Hempel's directions, and the oxygen must have been absorbed in a short time. Nevertheless, the eggs not only segmented, but they developed as far as normal eggs do in about fifteen hours after fertilization. A large blastoderm was formed which spread over a great part of the surface of the egg. 
In order to be able to compare these results with those obtained on the Ctenolabrus egg, I repeated the experiments on Fundulus, using the same method of replacing oxygen by hydrogen, and the same apparatus which had been used in the case of the Ctenolabrus egg.

The results obtained were in entire harmony with our earlier findings. When freshly fertilized eggs of Fundulus are introduced into the Engelmann chamber, and a vigorous stream of hydrogen is passed through it, the eggs divide not only once, but continue to do so for fifteen to twenty hours, until a blastoderm is formed which extends over the surface of the egg. The result was the same when the eggs were put in an Engelmann chamber and kept for two and one-half or three hours on ice, during which time they were exposed to a vigorous stream of hydrogen. When the eggs were then exposed to room temperature, segmentation at once began and continued in a regular manner. During the entire course of the experiment hydrogen was permitted to pass through the chamber.

As long as the number of the cleavage-cells was so small that they could be counted, it could be seen that development without oxygen occurred as rapidly as in oxygen. Whether this holds also for later stages when cleavage approaches the standstill cannot be determined, as the cells are then too small to allow one to count them. Not only cleavage, but also growth, of the blastoderm, that is to say, increase in area (at the expense of the yolk (?), 1903) occurs in the absence of oxygen. The blastoderm grows from a small area to a large area on the surface of the yolk.

If Fundulus eggs are allowed to remain more than twelve to fifteen hours in hydrogen, the cells nevertheless do not liquefy, as is the case in Ctenolabrus in the absence of oxygen. Even after twenty-four hours no such phenomena are observable in the Fundulus egg. I have shown in 
previous papers that such eggs do not lose their power of dividing even after being kept for three to four days without oxygen. On the other hand, I noticed a collection of the strongly refractive droplets in the furrows between the cells in Fundulus eggs also. Our observations on the mechanics of cell-division, therefore, seem to hold also for the Fundulus egg, only that the material for the surface layer of the Fundulus cells seems to be different chemically from that of the Ctenolabrus cells in that the latter in the lack of oxygen flows together into droplets, while the former undergoes no such structural changes.

On the other hand, the Fundulus egg is very sensitive to carbon dioxide. If a current of carbon dioxide is passed through the gas-chamber in which are contained the freshly fertilized Fundulus eggs, not a single cleavage occurs. Furthermore, the eggs which have resided for only four hours in such a current of carbon dioxide have lost their power of development for all time. This is of great importance in judging of the effects of lack of oxygen-it points to the possibility that the resistance of the protoplasm to lack of oxygen is not so very different in the Ctenolabrus egg from that in the Fundulus egg, and that only a secondary molecular change - the disintegration of the surface layer of the cells into a number of droplets - brings about a rapid destruction of the Ctenolabrus cells.

This possibility is supported by another fact. I have pointed out in an article, which I have already cited, the remarkable indifference of the Fundulus egg to the concentration of the sea-water. This year Professor W. W. Norman made similar experiments in my laboratory upon the Ctenolabrus egg. In these it was found that the Ctenolabrus egg is almost as insensitive to an increase in the concentration of the sea-water as is the Fundulus egg.

I should not like to conclude this section withont adding 
a word on the importance of comparative methods in physiology. If we had confined our experiments to the Ctenolabrus egg, a generalization of the facts observed would have been as follows: Cleavage is impossible without oxygen. Had we confined our experiments to the Fundulus egg, we should have come to the opposite conclusion. In reality, conditions are such that in some forms a cleavage is possible without oxygen, while in others it is impossible. The same may be said regarding protoplasmic motion. I do not as yet consider it as settled that every muscle is able to do a large amount of work without free oxygen.

IX. THE EFFECT OF THE REMOVAL OF OXYGEN ON THE SEGMENTATION OF SEA-URCHIN EGGS

If freshly fertilized sea-urchin eggs are introduced into a gas-chamber and a strong current of hydrogen is sent through it, one cleavage always occurs, and sometimes two. If, however, before beginning the actual experiment, all of the oxygen necessary for cleavage is driven out of the eggs and the gas-chamber (by placing the latter upon ice for two hours and sending a current of hydrogen through it), no cleavage occurs, even though we wait from three to four hours. If after this the eggs are again exposed to air, cleavage begins in about forty to fifty minutes. But all the eggs first divide into two cells, and only a few divide at once into three or four cells. The number of the latter is not greater in the experimental eggs than in the normal eggs of the same culture.' Such phenomena are very probably attributable to polyspermia. These facts show that in seaurchin eggs neither a division of the cell nor of the nucleus is possible without oxygen. In this particular they behave like the eggs of Ctenolabrus. We must now raise the question: Is the inability of cleavage in sea-urchin eggs also the consequence of molecular changes which are brought about by lack of oxygen? This, indeed, seems to be the case. 
If the eggs have divided into two or four cells, and the oxygen is then removed completely from them, the cell-limits become indistinct in about three hours. The cells then absorb water in consequence of the effects of lack of oxygen. The volume of the eggs increases, and the space within the membrane is soon filled uniformly with the protoplasm of the cleavage-cells. The outlines of the cell then become invisible, and the egg looks as if it had never divided. If oxygen is readmitted, the eggs cleave anew, if too long a time is not allowed to elapse. In many cases the old lines of cleavage reappear, but this is by no means always the case. The changes remind one of those in the eggs of Ctenolabrus, only that they occur more rapidly and more distinctly in the latter than in the eggs of the sea-urchin.

The surface of the cleavage-cells of the Arbacia is pigmented, and the pigment granules move upon the surface of the egg during cleavage. I do not doubt that by more careful study phenomena similar to those observed in the cleavage of the Ctenolabrus and the Fundulus eggs will be observed in the case of Arbacia also.

The fact has been mentioned that, in general, the cleavage of the Fundulus egg without oxygen occurs not only just as rapidly as under normal conditions, but even a little more rapidly, as stated in my paper on "The Relative Sensitiveness of the Fundulus Embryos in the Different Stages of Development against Lack of Oxygen." In that article, however, I attributed this difference in time to the increase in temperature brought about in sealing up the test-tubes used in the experiments. Since I again noticed these changes this year, first in the Ctenolabrus egg, and later in the Arbacia egg, in an Engelmann chamber - where there was, therefore, no considerable increase in heat - I decided to determine by more careful experiments whether this difference in time is indeed dependent entirely upon differences in temperature, 
or whether the altered metabolism in the initial lack of oxygen does not at first lead to a slight acceleration of cleavage. If the latter were correct, it would give a basis for the explanation of a very purposeful arrangement in organic nature, namely, the increase in respiratory activity in the lack of oxygen. For if lack of oxygen leads to such a universal change in metabolism that more energy is at first set free than under normal conditions, then the purposeful arrangement of the respiratory center is only a special case of a general property of protoplasm.

Yet the acceleration of cleavage in the Engelmann chamber might also be dependent upon an increase in temperature. One source of this increase in temperature might be sought in these experiments in the heat produced in developing hydrogen from zinc and sulphuric acid. The gas was passed through four wash-bottles before reaching the gas-chamber, yet it might nevertheless have caused an increase in the temperature in the gas-chamber. To render this impossible or less possible the gas generator was packed in a vessel with ice before beginning the experiment. From this the hydrogen was led through a bottle filled with chipped ice which was in turn again packed in ice. The first three wash-bottles were also kept on ice. The temperature of the last wash-bottle through which the gas passed before reaching the gas-chamber was carefully watched before and during the experiment. No increase in temperature was noted when the hydrogen was passed through it.

The same water was used for the eggs in the gas-chamber that was used for the control eggs. Every decrease in the temperature of the latter through evaporation of the water was carefully avoided, and their temperature carefully watched.

Cleavage in the eggs kept in the gas-chamber neverthe- 
less preceded that in the normal eggs by three or four minutes.

The experimental eggs as well as the control eggs were fertilized at the same time and in the same dish with a large amount of sperm. The process of driving out the oxygen by hydrogen was begun some ten or fifteen minutes after fertilization. About half an hour later cleavage occurred, usually first in the gas-chamber. At this time all the oxygen was probably not yet driven out of the eggs, so that we were dealing only with a partial lack of oxygen. This partial lack of oxygen, therefore, often brought about an acceleration of cleavage equal to 6 to 10 per cent. of the time necessary for the first cleavage. ${ }^{1}$ These experiments give one the impression that when lack of oxygen has reached a certain stage, a transitory increase in the development of energy occurs within the egg at first (through the formation of poisonous substances?). This increase in the development of energy, which, in the case of the respiratory center, is of enormous practical importance, therefore seems to appear also in such cases where its appearance is entirely unimportant, as in cleavage. I will not yet commit myself definitely to the statement that in case of a partial lack of oxygen a transitory acceleration of cleavage occurs; but to trace back the purposefulness of organized nature to the general chemical and physical properties of protoplasm seems to me much more promising than the assumption of natural selection.

If we summarize the results of these experiments on the effects of lack of oxygen on cleavage, we find that in the Fundulus egg, where in the absence of oxygen no dissolution of the cell-walls of the cleavage-spheres occurs, cleavage can continue for more than ten hours without oxygen; while in

1 I am still inclined to believe that, in spite of all the precautions, the hydrogen flad a slightly higher temperature than the air when it reached the egrgs. [1903] 
the Ctenolabrus, and eggs ${ }^{1}$ which cannot cleave without oxygen, the surface layer of the cleavage-cells is liquefied and the cells fuse together. The latter fact seems to indicate that cleavage does not occur in certain eggs, because without oxygen profound molecular changes occur, which, among other things, prevent the formation of a membrane or a specific surface film.

\section{ON THE EFFECT OF LACK OF OXYGEN ON CARDIAC ACTIVITY IN FISH EMBRYOS}

The older experiments on the effect of lack of oxygen on the activity of the heart have in part led to strange results. Tiedemann, for example, found that when the heart of frogs or salamanders is excised and kept under the bell of an air-pump, it ceased to beat in less than one minute when the air is rarified. ${ }^{2}$ Castell $^{3}$ came to more probable results. He found that when the heart is cut out of the body of a frog and kept in an indifferent medium in the absence of oxygen, it may continue to beat for an hour. In the experiments of Pflüger and Aubert, which have already been mentioned, the heart continued to beat after all the spontaneous movements of the animal had long ceased.

The older authors had discussed the question as to whether oxygen does not have a direct stimulating effect upon the heart. This would, of course, explain why the heart ceases to beat when oxygen is lacking. Castell, however, showed that a heart which has ceased to beat in an atmosphere free from oxygen will also not beat when stimulated by other means. The papers which have been cited in the introduction give a more rational explanation of the rôle of oxygen

1 This phenomenon is less distinct, and therefore not so certain, in the egg of Arbacia as in that of Ctenolabrus. Driesch questions it in the sea-urchin egg, but I am not certain that his experiments are identical with mine. [1903]

2 Archiv für Anatomie und Physiologie, 1847, p. 490.

3 Ibid., 1854, p. 226. 
than that furnished by the assumption that the oxygen "stimulates" the heart.

I was especially interested in comparing the effects of lack of oxygen on the beat of the heart in Ctenolabrus and Fundulus embryos. Does the same difference in behavior toward lack of oxygen exist here as in regard to cleavage?

The heart begins to beat and the circulation is established in Ctenolabrus embryos as early as forty-eight hours after fertilization. If such forty-eight-hour-old embryos, which are still contained within the eggs, are introduced into a gas-chamber through which a current of hydrogen is passed, the heart usually comes to a standstill in from three to ten minutes after the current of gas is turned on. The activity of the heart does not, however, gradually fall to zero, but the heart comes to a standstill suddenly when the number of heart-beats has decreased but little or not at all. In one case the heart beat about 90 times a minute before the hydrogen was admitted. Hydrogen was then passed through the gas-chamber, and after four minutes the heart still beat 89 times; two minutes later it beat 78 times, and in the following minute 77; in the next minute the heart came to a sudden standstill. After hydrogen had been passed through the gas-chamber for only seven minutes, and when the number of heart-beats had fallen only from 90 to 77 - a slight decrease only - the heart suddenly stood still; at that time blood was still circulating beautifully.

In a second experiment the number of the heart-beats was 108 per minute at the beginning of the experiment. Two minutes after turning on the hydrogen gas the heart beat 105 times, and three minutes later 108 times a minute. During the next minute the heart stood still after having beaten 23 times in the first eighteen seconds of that minute. The heart stood absolutely still for four minutes, after which it gave a few weak pulsations. For the next three minutes 
it again stood still, after which the heart beat rhythmically for one minute (38 beats in a minute), when it again ceased. A few irregular pulsations followed, and then everything was over. Sixteen minutes after turning on the current of hydrogen the heart had come to a complete standstill, but the embryo itself still moved at this time, and even five minutes after the heart and the circulation had ceased entirely the embryo still moved!

In a third experiment the current of hydrogen was turned on at 11:26 A. м. The number of beats was 90 per minute; in the following minute it was 81 , and in the third minute the heart came to a sudden and permanent standstill. In a fourth experiment the current of hydrogen was started at 10:03 A. M. The number of heart-beats was 100 per minute. The following table indicates the course of the experiment:

\begin{tabular}{|c|c|c|c|c|c|c|c|c|}
\hline $10: 03$ & - & - & - & - & 100 & bea1 & per & ninute \\
\hline $10: 04$ & - & - & - & & 102 & “ & “" & " \\
\hline $10: 05$ & - & - & - & - & 100 & “ & " & " \\
\hline $10: 06$ & - & & . & - & 96 & " & “ & “ \\
\hline $10: 07$ & - & - & - & - & 98 & " & “ & " \\
\hline $10: 08$ & - & - & - & - & 90 & " & " & " \\
\hline $10: 11$ & - & - & - & - & 60 & " & " & .6 \\
\hline $10: 12$ & - & - & - & - & 54 & " & “ & " \\
\hline $0: 1$ & & & & & 54 & " & ، & " \\
\hline
\end{tabular}

The heart then came to a sudden standstill. Three minutes later the heart again beat twice; shortly after this it beat regularly for one minute (39 times per minute). The heart then again stopped; a few scattered beats followed, and at 10:25 A. M. the heart came to a permanent standstill.

When the embryos whose hearts had come to a standstill were returned, after not too long a time, to water containing oxygen, resuscitation of the heart followed, and this the earlier, the shorter the time the embryo had remained in the atmosphere free from oxygen. If the eggs remained for one to one and a half hours in the gas-chamber, they became 
opaque and sank to the bottom. Twenty-five minutes after turning on the hydrogen the changes which we have described in detail above-namely, the appearance of the strongly refractive droplets - were often clearly visible.

If now we ask for the cause of the rapid and sudden standstill of the heart of Ctenolabrus embryos when deprived of oxygen, we must admit, first of all, that a failure of the energy which is supplied perhaps by processes of oxidation cannot be the cause. For, since the oxygen is replaced by hydrogen only gradually, the number of heart-beats should under these circumstances also decrease only gradually until a minimum is reached. The behavior of the heart was, however, entirely different. The heart usually came to a standstill without a noteworthy decrease in the number of heartbeats; sometimes a decrease was noted. For the same reasons the view that in three to ten minutes after turning on the current of hydrogen all the potential energy present in the heart has been used up is also to be set aside. After the heart had ceased to beat, the entire animal still executed spontaneous movements, and the heart remained generally active in case of lack of oxygen longer than the rest of the body of an animal. $\quad$ The rapid and sudden standstill of the heart of Ctenolabrus is the consequence either of a sudden poisoning, or of a structural change in the heart brought about by the removal of oxygen. It might also be that the poisonous effect consists only in bringing about molecular changes. The experiments on the cleavage of the Ctenolabrus egg showed that a change occurs in the cell-walls in consequence of which they break up into droplets. We must assume that these changes are brought about by the beginning lack of oxygen, or the metabolic products formed in consequence of this lack of oxygen. Might it not be possible that a liquefaction of solid elements and the formation

1 Miss Moore has since found that in young fish whose respiratory and sponta. neous motions have ceased the heart still continues to beat for hours. [1903] 
of droplets hinders the production or the transmission of molecular movements, and in this way brings about the sudden standstill of the heart? This idea would also harmonize very well with the fact that the heart comes to a standstill as suddenly and as unexpectedly as death ensues from embolism. It would also be in harmony with this idea that after the sudden standstill of the heart a few occasional heartbeats may yet appear. We will, however, not enter farther into the field of hypotheses, but rather attempt to see how the heart of Fundulus behaves in the lack of oxygen.

Numerous experiments on embryos from four to ten days old (the embryos do not hatch until after the twelfth day) showed without exception the following behavior of the heart in the case of lack of oxygen:

During the first ten to twenty minutes after the hydrogen is turned on through the gas-chamber, the number of heartbeats does not decrease. A transitory acceleration even occurred, which, however, was brought about through a rise in temperature caused by passing the hydrogen gas through the gas-chamber. This acceleration did not occur when I packed the hydrogen generator in ice. But the decrease in the amount of oxygen contained in the Fundulus egg, which occurs during the first twenty minutes and which causes the heart of the Ctenolabrus embryo to stand still, has no effect upon the rate of the heart of the Fundulus embryo.

Then follows a period of steady decrease in the number of heart-beats, which continues for about one and one-half hours. The decrease occurred most rapidly at first and then more slowly. During this period the number of heart-beats fell from about 120 or 100 a minute to about 20 per minute. This period corresponds, it seems to me (and we shall find further proofs for this idea later), to the period of progressive decrease in the oxygen necessary for the oxidations in the heart. 
When the number of the heart-beats has decreased to the minimum of about 20 per minute, the heart continues to beat at this rate for about eight to ten hours in an uninterrupted and regular manner, until at the end of this time it comes to a standstill. Since our earlier experiments rendered

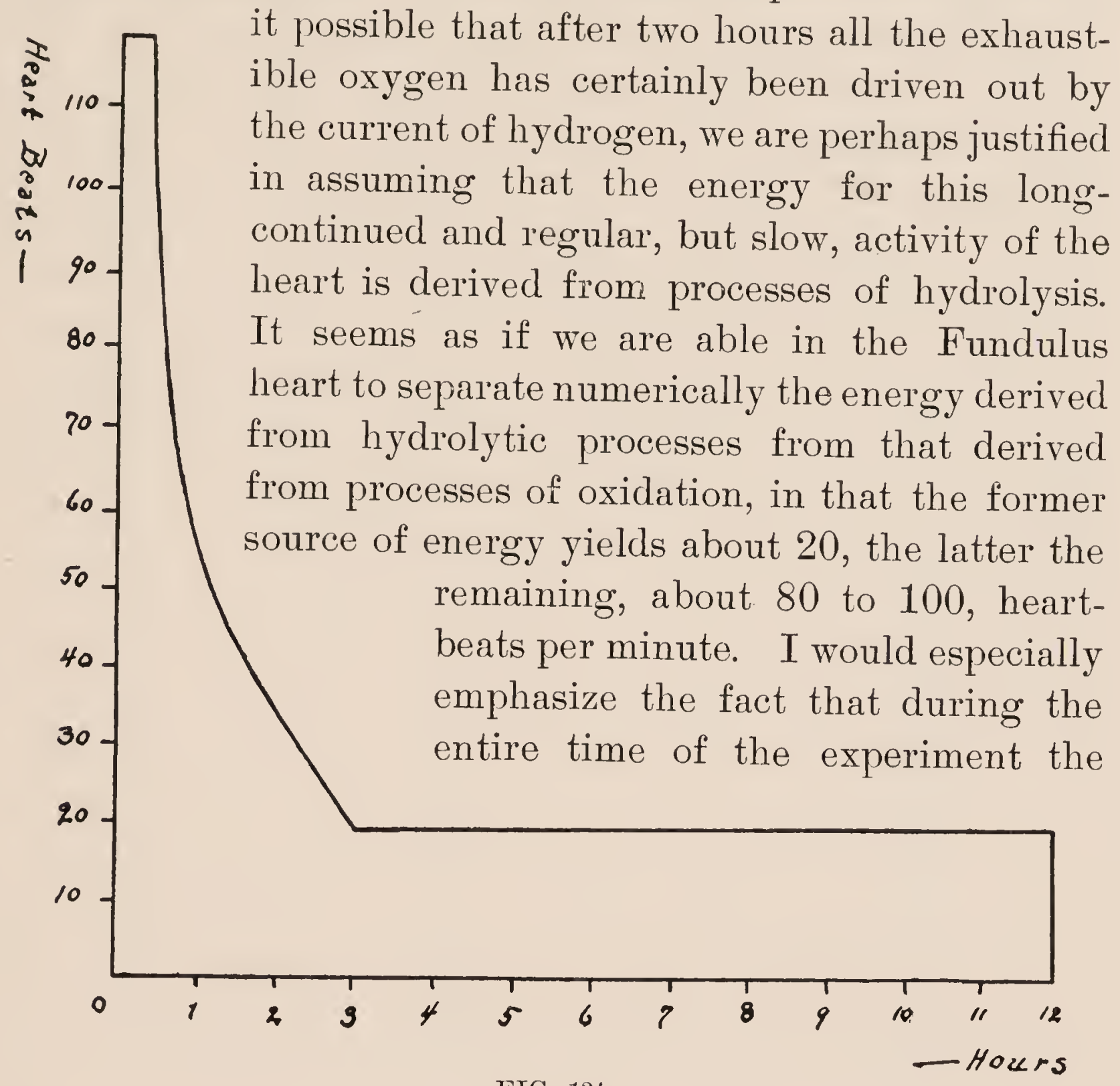

FIG. 124

current of hydrogen was passed through the gas-chamber uninterruptedly, and that in consequence every action of the carbon dioxide had been shut out in these experiments, as in those upon the Ctenolabrus embryo.

We shall now describe a few of the individual experiments. In one case the hydrogen current was turned on at $8: 42$ A. . The number of heart-beats was 108 to 114 per 
minute. This number remained constant until about 9:08. (The current of hydrogen was not as vigorous as usual.) At $9: 12$ the number of heart-beats was 96 ; at 9:30 the number was 69 ; at 10 the number was 48 ; and at 11 it had fallen to 27. At 11:25 the heart beat 23 times per minute; at 11:40 it beat 20 times per minute; after which the number of beats varied between 20 and 23 per minute, until 8:45 P. M.; in other words, more than nine hours. The curve of Fig. 124 illustrates the condition of affairs better than description. The curve is typical and may be looked upon as representing any one of these experiments. Only the absolute values varied with different individuals and with the temperature.

In another experiment the current of hydrogen was turned on at 3:06 A. M. The number of heart-beats was 120. At $3: 17$ the heart beat 126 times, after which the number decreased, as shown in the following table:

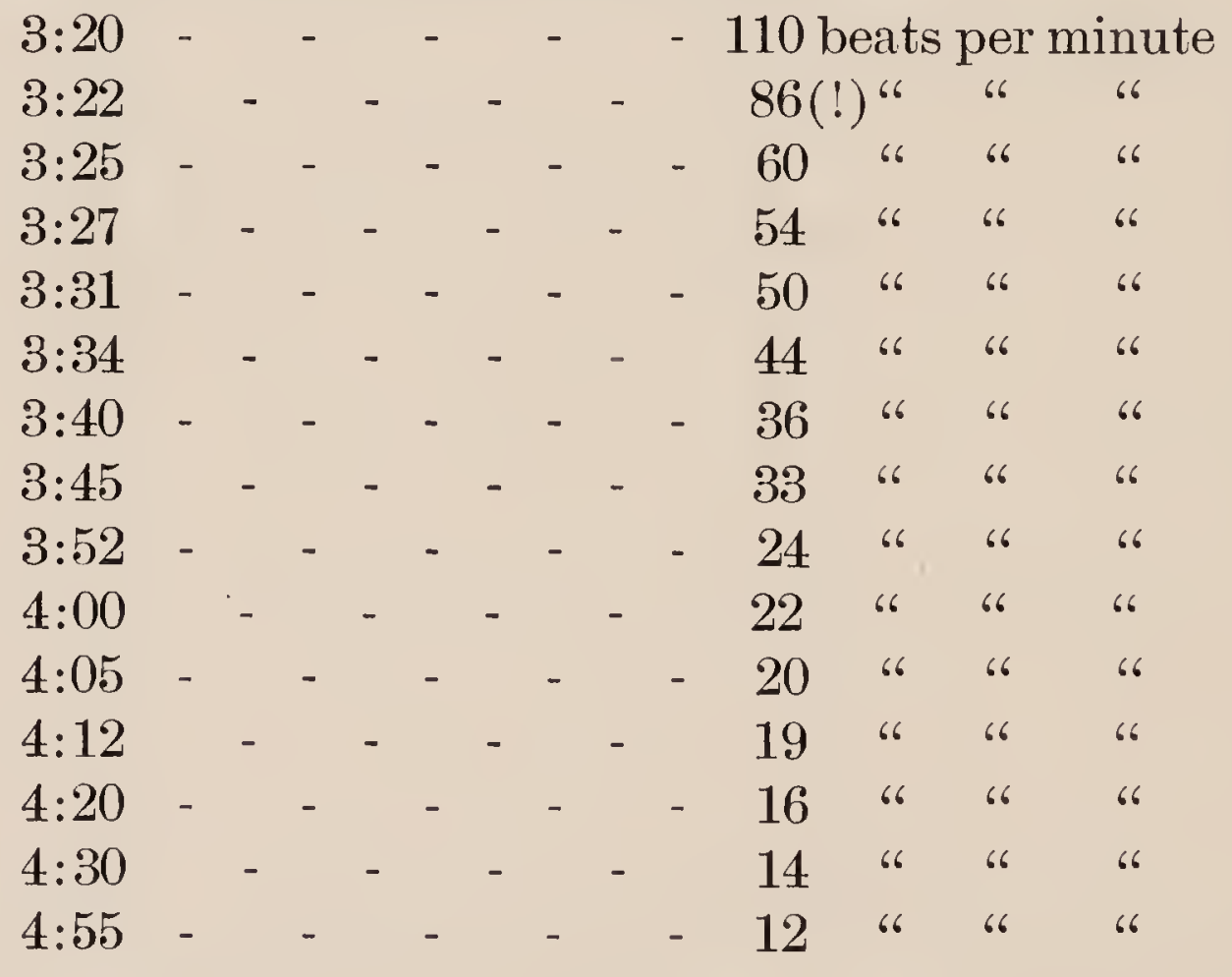

This rate continued unchanged until 9:50, when the experiment was brought to a close. It is readily seen how much more rapidly the decrease occurs at first than later. Fig. 125, which illustrates the beginning of this experiment, shows this very strikingly. 
I have repeated this experiment eight times, always with the same result. It was of importance now to determine whether the number of heart-beats increases, and how much it increases, when a heart which has attained its minimum rate in hydrogen is again exposed to the oxygen of the air.

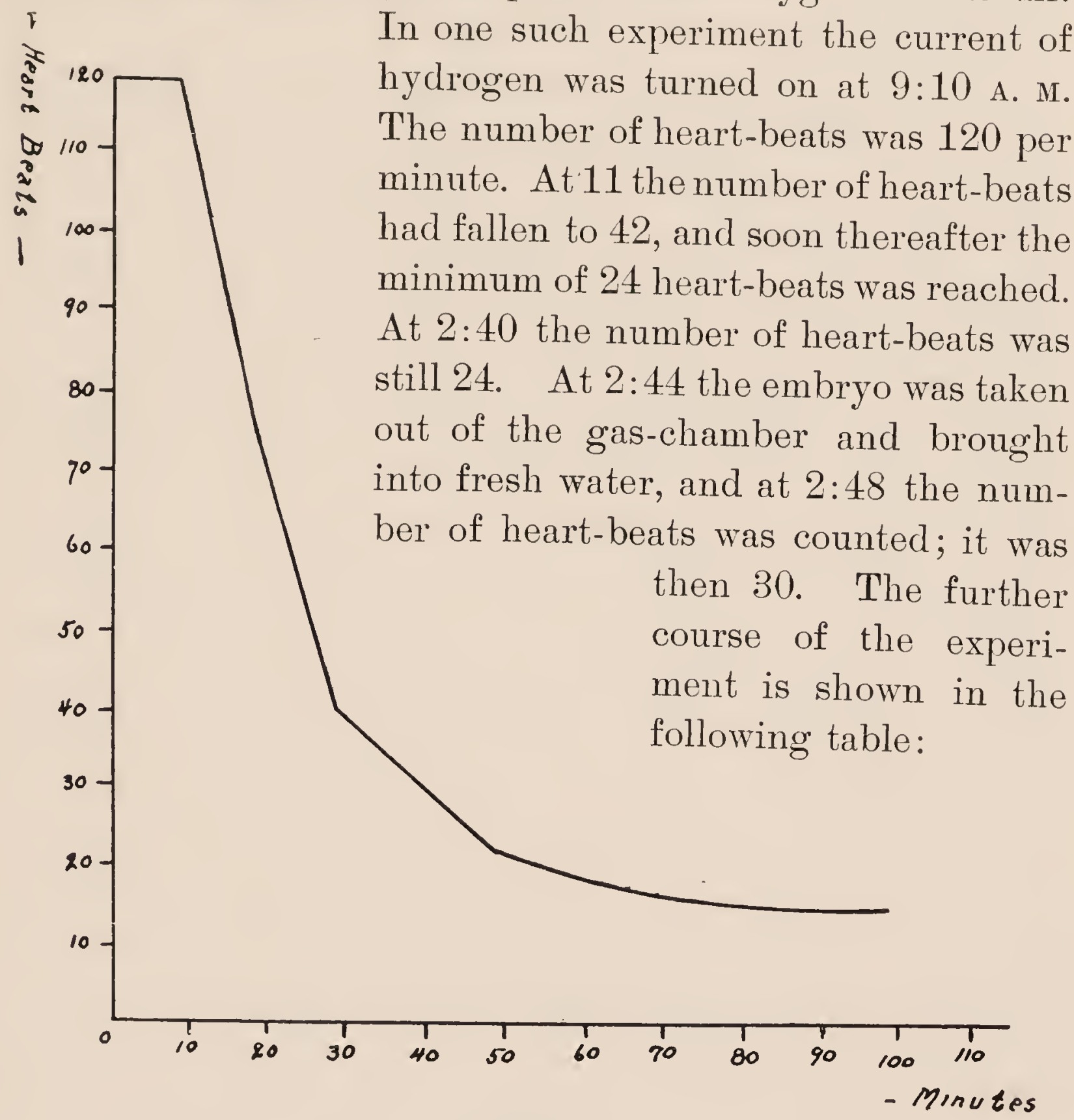

FIG. 125

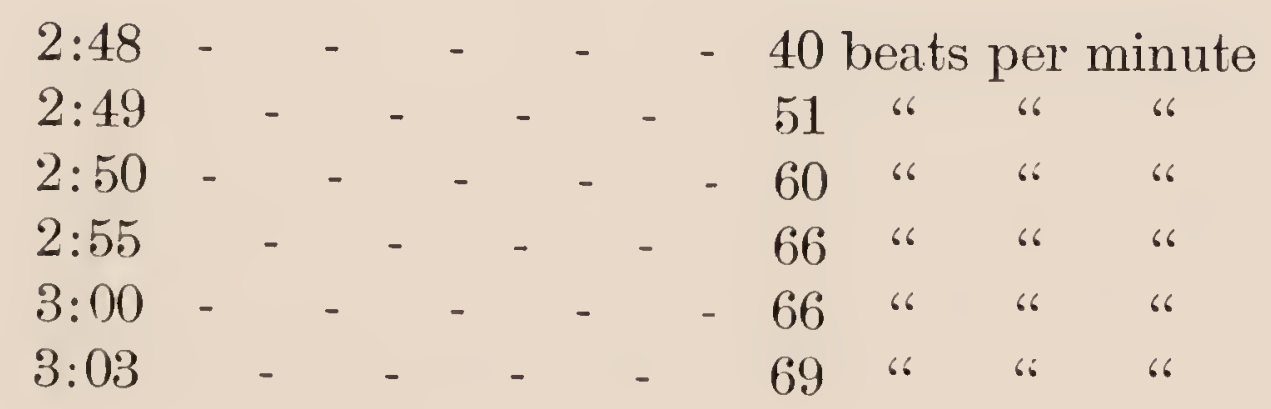




\begin{tabular}{|c|c|c|c|c|c|c|c|c|c|c|c|}
\hline $3: 05$ & - & - & & $=$ & & - & & 75 & beat & per & minute \\
\hline $3: 08$ & & & - & & - & 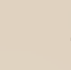 & & 81 & 66 & 6 & 66 \\
\hline $3: 15$ & - & - & & - & & - & & 84 & $" 6$ & 66 & 66 \\
\hline $3: 25$ & & & - & & - & . & 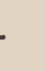 & 96 & 66 & 6 & 66 \\
\hline $3: 35$ & - & - & & - & & - & & -102 & 66 & 6 & 6 \\
\hline $3: 47$ & & & - & & - & . & & 111 & 66 & 66 & 66 \\
\hline$: 53$ & - & - & & - & & - & & 120 & 6 & 66 & 66 \\
\hline
\end{tabular}

This rate continued until $5: 10$, when it increased to $132{ }^{1}$ This experiment, which I repeated several times with the same result, shows that the decrease in the number of heartbeats when the heart is deprived of oxygen is dependent chiefly upon the decrease in the energy furnished by oxidation and not upon the formation of poisonous substances. The fact that the minimum number of heart-beats continues a very long time without oxygen also speaks against the latter idea.

We can make use of still another method to determine what proportion of the heart-beats in the Fundulus embryo depends upon oxidations, and what proportion upon processes of splitting. By placing the gas-chamber upon ice and passing a current of hydrogen through it, we are able to drive out the oxygen, while the processes of hydrolysis are at the same time reduced to a minimum through the lowering of the temperature. In one experiment I passed the hydrogen through the gas-chamber for two hours, while keeping it on ice. The hearts were then removed from the ice, but the current of hydrogen was maintained. At room temperature the number of heart-beats, which at the beginning of the experiment had been 117, rose to 87 (in twelve minutes), to descend again to 36 in the course of the next hour. Forty minutes later the minimum of 21 was attained, at which rate the heart continued to beat for seven hours. Toward the last a slight increase occurred

1 This experiment shows that the oxygen diffuses comparatively rapidly into the egg. [1903] 
in the number of beats. I had expected that the number of heart-beats would be only the minimal one after removing the gas-chamber from the ice. Possibly all the oxygen had not been driven out. I therefore repeated the same experiment, but allowed the gas-chamber to remain for three hours on the ice. This time I expected that at room temperature the number of heart-beats would only reach the minimum which corresponded to the temperature. But this time also the number of heart-beats rose in six minutes to 66 , after which the rate decreased steadily. One hour later the heart beat 42 times, and after thirty-five minutes the minimum of 24 was reached. I do not doubt that after passing a vigorous current of hydrogen through the gas-chamber for three hours all the oxygen is exhausted from the egg. If this assumption is correct, these experiments can be made to harmonize theoretically with the results obtained earlier only by assuming that the processes of hydrolysis do not occur with uniform intensity, but that they occur much more rapidly at first when the oxygen is first withdrawn (or perhaps also under the ordinary conditions of oxygen supply) than in the continued lack of oxygen. ${ }^{1}$

It is, moreover, to be noted that the data necessary for calculating the work of the heart are lacking in these experiments. Only by assuming that these data are the same in the presence of oxygen as in its absence can conclusions be drawn as to the behavior of the two sources of energy. If we make this assumption, we come to the conclusion that of all the energy which is used up by the Fundulus embryo in normal heart-activity, that much at least which corresponds to the minimal number of heart-beats in the lack of oxygen is dependent upon processes of hydrolysis. This number is about one-sixth or one-fourth of the total number of heart-beats which occur under normal conditions of [1903]

1 Perhaps in this case the effects of poisonous substances are to be considered. 
oxygen supply and at the same temperature. When, however, we consider the results of the experiments carried on in the cold, we come to the conclusion that in the presence of oxygen the proportion of energy obtained through processes of splitting may be much greater than this; it may then amount to 50 or 70 per cent. of the work done by the heart.

The behavior of the heart of a Fundulus embryo in carbon dioxide is of interest in so far as it shows that carbon dioxide is just as poisonous in this case as upon the heart of the Ctenolabrus. While the Fundulus heart continues to beat for twelve hours, and even longer, when the oxygen is driven out by hydrogen, the ventricle ceases to beat as early as twelve minutes after passing carbon dioxide through the gas-chamber. Only the auricle continues to beat, and the circulation soon comes to a stop. The contractions become weaker and less numerous. In one experiment the heart beat 96 times per minute at the beginning of the experiment, 54 times after eight minutes, 45 times after ten minutes, and 42 times after twenty minutes. The heart then ceased to beat entirely for long periods of time, and thirty-two minutes after turning on the carbon dioxide the heart stood still. In other experiments the heart did not cease to beat until after one and one-half hours. When the heart is exposed to the poisonous effect of $\mathrm{CO}_{2}$ and ceases to beat even after one hour, the heart begins to beat again when the carbon dioxide is replaced by air. The resuscitation of the heart is as follows: The auricle recovers more rapidly than the ventricle, and the latter at first beats a less number of times than the former. In one experiment a heart which had come to a standstill was exposed to air at 10 A. M. At 10:06 the auricle beat 24 times per minute, while the ventricle was still quiet. The ventricle did not begin to contract until the next minute, and the number of auricular contractions was 33 a minute at this 
time. At 10:23 the auricle beat 72 times, while the rentricle beat 42 times per minute. The ventricle often contracted only once to every two, or even at times three, auricular contractions. At 10:35, however, the rate of the ventricular and the auricular contractions was the same, namely, $8 t$ per minute, and from then on they continued the same. These phenomena, which are characteristic of all experiments with carbon dioxide, were never observed in replacing the air by pure hydrogen.

In another series of experiments I permitted $\mathrm{CO}_{2}$ and hydrogen to pass alternately through the gas-chamber. In one case I turned on the hydrogen at 8:30 A. M. At 10:30 the heart beat 24 times per minute; at 10:31 the hydrogen current was interrupted and the $\mathrm{CO}_{2}$ current was turned on. (Through a simple T-tube connection and a pair of pinch cocks it was possible to pass either the hydrogen or the $\mathrm{CO}_{2}$ through the gas-chamber at will, without admitting air.) In a few minutes the ventricle ceased to beat and the circulation stopped. After an hour the current of carbon dioxide was interrupted, and hydrogen was again passed through the chamber. After forty minutes the ventricle again began to beat; the number of its beats was $2 t$, and remained so until death. By replacing the $\mathrm{CO}_{2}$ by hydrogen it is therefore possible to do away with the poisonous action of the former. This experiment demonstrates very nicely a fact which is perhaps doubted by no one: that carbon dioxide and lack of oxygen have entirely different effects, which in ordinary cases of asphyxia are added together. ${ }^{1}$ In this way it is possible by passing through the chamber a current of pure hydrogen gas to bring to life again a ventricle which has been asphyxiated in carbon dioxide.

1 It also demonstrates very nicely the possibility that other non-volatile poisonous substances may be formed, by lack of oxygen, which are destroyed again when oxygen is again admitted. The phenomena of fatigue may belong to this categrory. $[190: 3]$ 
Finally, it was of interest to compare the resuscitating effect of air with the resuscitating effect of hydrogen. Fundulus embryos were introduced into two gas-chambers. At the beginning of the experiment the heart under observation in one of the chambers beat 90 times a minute; that in the other, 96 times a minute. Hydrogen was passed through the chambers, and after an hour and fifty minutes the frequency of the heart-beats had fallen in both cases to 18 per minute. In place of the hydrogén, carbon dioxide was then passed through the chambers. In fifteen minutes the ventricles stopped beating, and the pulsations of the auricles became much weaker. After 45 minutes one of the hearts was apparently dead, while the auricle of the other still beat 18 times a minute, though the beats were scarcely perceptible. One of the gas-chambers was then opened and the embryo exposed to the air, while in the second chamber the $\mathrm{CO}_{2}$ was replaced by hydrogen. After fifteen minutes the heart which had been apparently dead and which was exposed to the hydrogen beat 24 times a minute, but only the auricles contracted. Both the auricle and the ventricle of the heart which was exposed to the air beat 60 times. Two hours later the heart beat 30 times per minute in the hydrogen, but the contractions were still limited to the auricle, while the heart exposed to the air beat 72 times. When a little later I exposed the embryo kept in the hydrogen to air, the ventricle did not recover. The number of auricular contractions did rise within fifteen minutes from 18 to 54 , but shortly thereafter the entire heart ceased to beat. In resuscitating a heart poisoned by $\mathrm{CO}_{2}$, oxygen is therefore more effective than the simple removal of the $\mathrm{CO}_{2}$ by hydrogen. We are not able to explain why the ventricle ceases to beat when exposed to carbon dioxide sooner than the auricle. We meet with an entirely different relation between car- 
diac activity and oxygen in the larve of a fresh-water mussel (Cyclas). In this animal the frequency of the heartbeat steadily decreases from 50 heart-beats to 0 in the course of one and one-half hours in an atmosphere of hydrogen (at $24^{\circ}$ C.). In this case, therefore, we have neither a sudden standstill of the heart without an appreciable decrease in the frequency, as in Ctenolabrus, nor a long-continued steady beat of low frequency, as in Fundulus, but a decrease in cardiac activity which runs parallel with the removal of oxygen, as if processes of oxidation are the sole source of energy for the activity of the heart.

XI. ON THE TRANSFORMATION OF NEGATIVELY HELIOTROPIC ANIMALS INTO POSITIVELY HELIOTROPIC THROUGH LACK OF OXYGEN

A series of papers have proved that it is possible to change the sign of heliotropism in certain animals at will through external conditions. ${ }^{1}$ It is an easy matter, for example, to render negatively heliotropic Copepods positively heliotropic by cooling, and to keep them permanently positively heliotropic at a low temperature; while it is also possible to render positively heliotropic Copepods negatively heliotropic by an increase in temperature. The same experiments can be made on larvæ of Polygordius. In order to determine the cause of this change in the sign of heliotropism, and also the conditions upon which the latter depends, I tried to see whether other conditions could bring about similar changes. Groom and I had previously found that the positively heliotropic Nauplii of Balanus perforatus rapidly became negatively heliotropic when exposed to strong light.

I also found that the same effect can be produced upon Copepods and Polygordius larvæ by properly diluting the

1 Groom und Loeb, Biologisches Centralblatt, Vol. X; Loeb, Vol. I, pp. 265 ff. 
sea-water as by increasing the temperature, while a proper increase in the concentration of the sea-water brings about the same effect as cooling.

The majority of Copepods were, immediately after being caught, positively heliotropic. It seemed as if the majority of the negatively heliotropic Copepods belonged to one and the same species. When the Copepods were allowed to remain for a long time in a vessel containing sea-water, the number of negatively heliotropic animals decreased, becoming positively heliotropic with time, while the reverse change occurred only rarely. The experiments on the effect of lack of oxygen were made under a small bell-jar, the contents of which were separated from the air on the outside by mercury. Two tubes extended into the bell-jar, one of which conducted the hydrogen into the bell, while the other conducted it away from the bell. Two vessels were placed under the bell-jar, of which the one contained freshly selected positively heliotropic Copepods, while the other contained negatively heliotropic Copepods. While the positively heliotropic animals remained positively heliotropic during the course of the experiment, the negatively heliotropic Copepods within fifteen to twenty minutes after the hydrogen was turned on began, in part, to leave the room side of the vessel and to distribute themselves irregularly throughout the vessel, in part to collect at the window side of the vessel. The number of animals collected near the window steadily increased, while the number of Copepods at the room side of the vessel steadily decreased. In about thirty to forty-five minutes after the current of hydrogen had been turned on all the Copepods lay quietly on the bottom of the vessel. The Copepods which had from the beginning been positively heliotropic died at the side of the vessel nearest the source of light. Most (if not all) of the Copepods which had at first been negatively heliotropic 
were also found at the window side of the vessel. A second small collection occurred in the middle of the vessel, while the room side of the vessel was entirely vacated. The animals usually did not become positively heliotropic until shortly before they became motionless. This explains why the conversion of the negatively heliotropic into the positively heliotropic animals through lack of oxygen cannot be obtained with the precision and elegance with which the change can be obtained by cooling. In the latter case the animals retain their full power of movement; in the former the transformation does not occur until the animals have suffered from lack of oxygen. But even then the phenomenon is so striking that it might be used as a demonstration experiment. I have repeated the experiment eight times with the same result. At first it seemed to me as if the negatively heliotropic Copepods died more rapidly in the absence of oxygen than those which were positively heliotropic from the beginning. This finding, however, was not borne out in every case.

When the experiment was interrupted early, at a time when the animals first began to become positively heliotropic, and air was then admitted, the Copepods which had become positively heliotropic again became negatively heliotropic.

The remarkable effects, which we have described here, of lack of oxygen on the sense of heliotropism, are, of course, not confined to Copepods. I made similar experiments upon the negatively heliotropic marine Isopods, the majority of which also become positively heliotropic in less than two hours when oxygen is withdrawn. These experiments will be continued.

We see, therefore, that lack of oxygen has the same effect upon the sense of heliotropism as cooling or increasing the concentration of the sea-water. Araki has shown that by cooling the chemical effects of lack of oxygen can 
be brought about, and it is therefore possible that the positive heliotropism in both cases is determined by the same chemical conditions. It must be left for further experiment to decide this point.

XII. ON CHANGES IN PIGMENT CELLS IN LACK OF OXYGEN

It is a definitely established fact that the pigment cells in the skin of the frog become lighter after death. This lightening is brought about, as Biedermann has found, ${ }^{1}$ by the fact that the coloring matter collects into small clumps. A piece of the skin which has been deprived of its circulation shows the same changes.

In the transparent portions of the skin which can be studied microscopically-such, for example, as the web of the amputated foot of Rana temporaria - it can easily be seen how the muchbranched pigment cells which follow the course of the capillaries gradually change their form, in that the coloring matter moves toward the center of the cell until finally all the pigment is collected into clumps (p. 175).

Increase in the carbon dioxide cannot be the cause of this change in the pigment cells, for Biedermann found that the skin does not become lighter when the frog is poisoned with $\mathrm{CO}_{2}$. Biedermann believes that the cause is probably to be found in the decrease in the amount of oxygen.

The surface of the yolk-sac of the Fundulus embryo is studded with a large number of black and reddish-yellow pigment cells, which are at first distributed irregularly, but which later, as I have shown, ${ }^{2}$ are compelled to creep upon the blood-vessels and surround them. With this the first physiological cause was furnished for the marking of an animal. Since then other authors have also found that the course of the embryonic blood-vessels determines the marking of the embryo.

1 Pflügers Archiv, Vol. LI.

2 Journal of Morphology, 1893. 
The black and red pigment cells can be distinguished from each other, not only by their color, but also by their form. The latter send out a large number of thin pseudopodia-like processes which are never found in the black pigment cells. In the experiments on the effect of lack of oxygen on the cardiac activity of the Fundulus embryo, it was noticed that the originally dark yolk-sac gradually became lighter in color when exposed to hydrogen for a long time. The pigment cells can be observed very carefully with the microscope, and I expected to observe the same phenomena that Biedermann observed in frogs. This was, however, not the case. It was found in the course of a series of experiments that the dark pigment granules and the black cells gradually disappear the longer the current of hydrogen is kept up, and that the collection of the pigment in the center of the cell does not occur.

The changes in the red pigment cells in lack of oxygen are of a somewhat different nature. The lightening of the color often occurs in this case also. Besides this, however, the cells become smaller. The tips of the cell-processes break off, remaining visible at first as tiny droplets, which disappear later. As this process continues, the pigment cells gradually become smaller.

These changes remind one of the fact that certain dyes become colorless when reduced. In our experiments it might also be possible that the discoloration of the black pigment is a result of a reduction, which does not occur in the presence of atmospheric oxygen.

\section{CONCLUDING REMARKS}

It seems to me that the most important result of the foregoing experiments consists in the proof which has been brought forward that in certain cases at first molecular. and later morphological, changes are brought about in cells 
through lack of oxygen, which in their turn are the cause of the suspension of life-phenomena. This has been proved for the process of cleavage in the Ctenolabrus egg. The cleavage-cells of Ctenolabrus are dissolved again and fuse together when oxygen is removed. These changes are not, however, an evidence of death, for as soon as such a fused blastoderm is again exposed to air it begins to divide anew. On the other hand, these molecular changes are sufficient to hinder cleavage. The cleavage-cells of the Arbacia egg seem to suffer similarly in the lack of oxygen, although the changes are much less marked. We find that here also cleavage is impossible without oxygen. Yet lack of oxygen does not bring about the same sort of molecular changes in the Fundulus egg as in the Ctenolabrus egg, and corresponding with this difference cleavage may also go on without oxygen for many hours in Fundulus.

It is also possible that such molecular changes as are brought about by the lack of oxygen in the cell are also the cause of the cessation of other life-phenomena; for example, the beat of the heart (and the activity of the respiratory center). We thus find that in Ctenolabrus, where the first cleavage-cells suffer such profound structural changes through lack of oxygen, the heart of the embryo comes to a standstill very rapidly and suddenly through lack of oxygen before a marked decrease has taken place in the frequency of the heart-beats; while the heart of the Fundulus, whose cells suffer no such structural changes, continues to beat for many hours without oxygen. Since the chemical energy set free in the cells must first be converted into molecular energy in order to bring about the physiological function, it is clear, a priori, that not only a decrease in the supply of the chemical energy, but any structural change which renders impossible the conversion of chemical energy into the molecular energy necessary for the activity of the tissue, 
must also lead to a standstill in the particular life-phenomenon under observation. We find that both possibilities are, indeed, encountered. Further investigation may possibly be able to show that the sudden cessation of life-phenomena through lack of oxygen may generally be attributable to structural changes. Were this the case, it might point the way, perhaps, to a clearer understanding of the action of many poisons. 


